
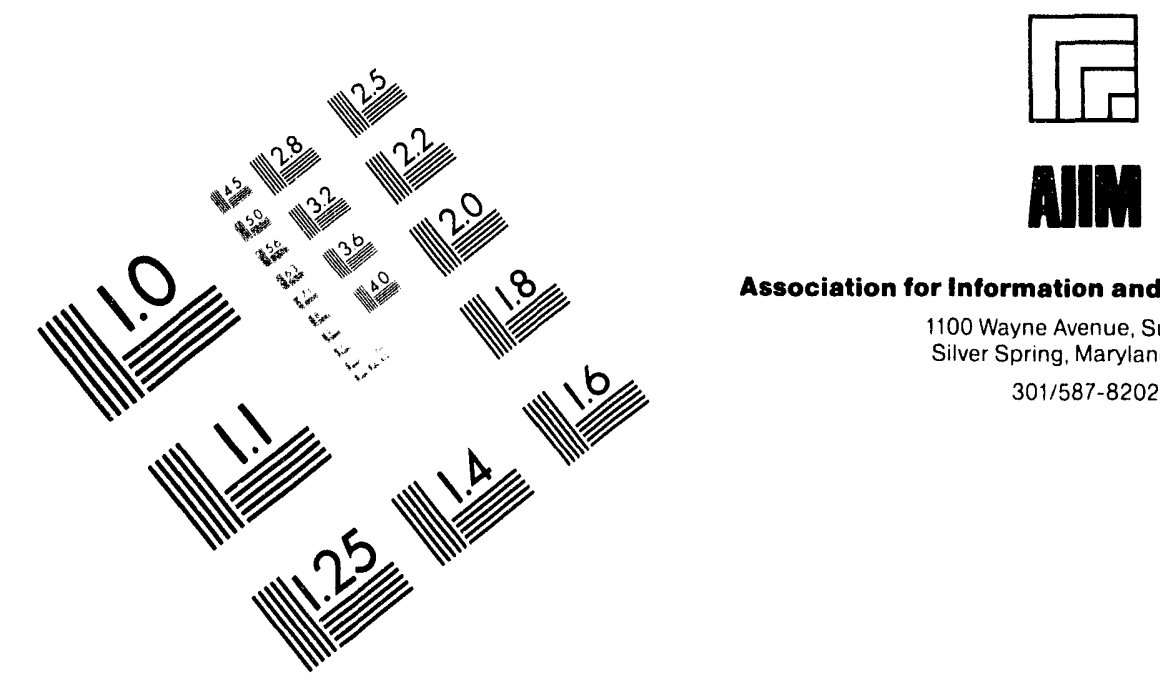

Association for Information and Image Management

1100 Wayne Avenue, Suite 1100

Silver Spring, Maryland 20910

301/587-8202

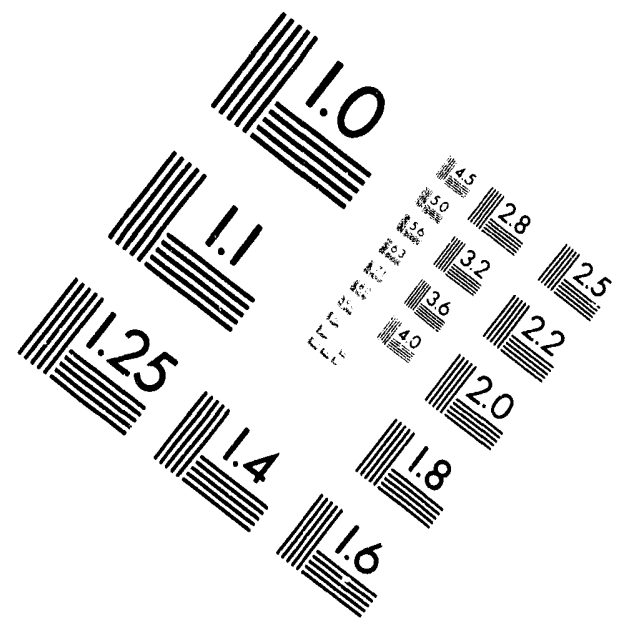

Centimeter

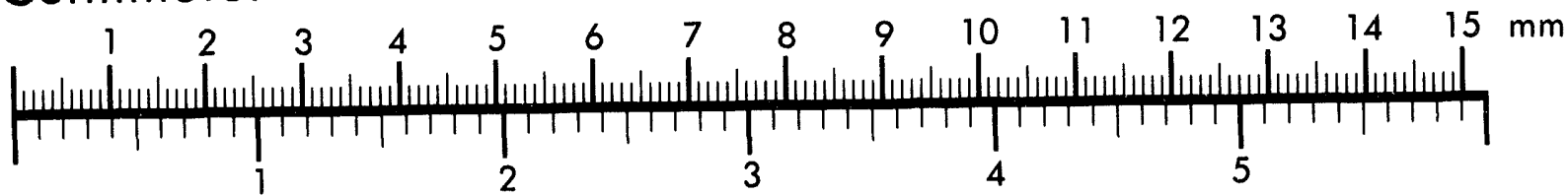
Inches
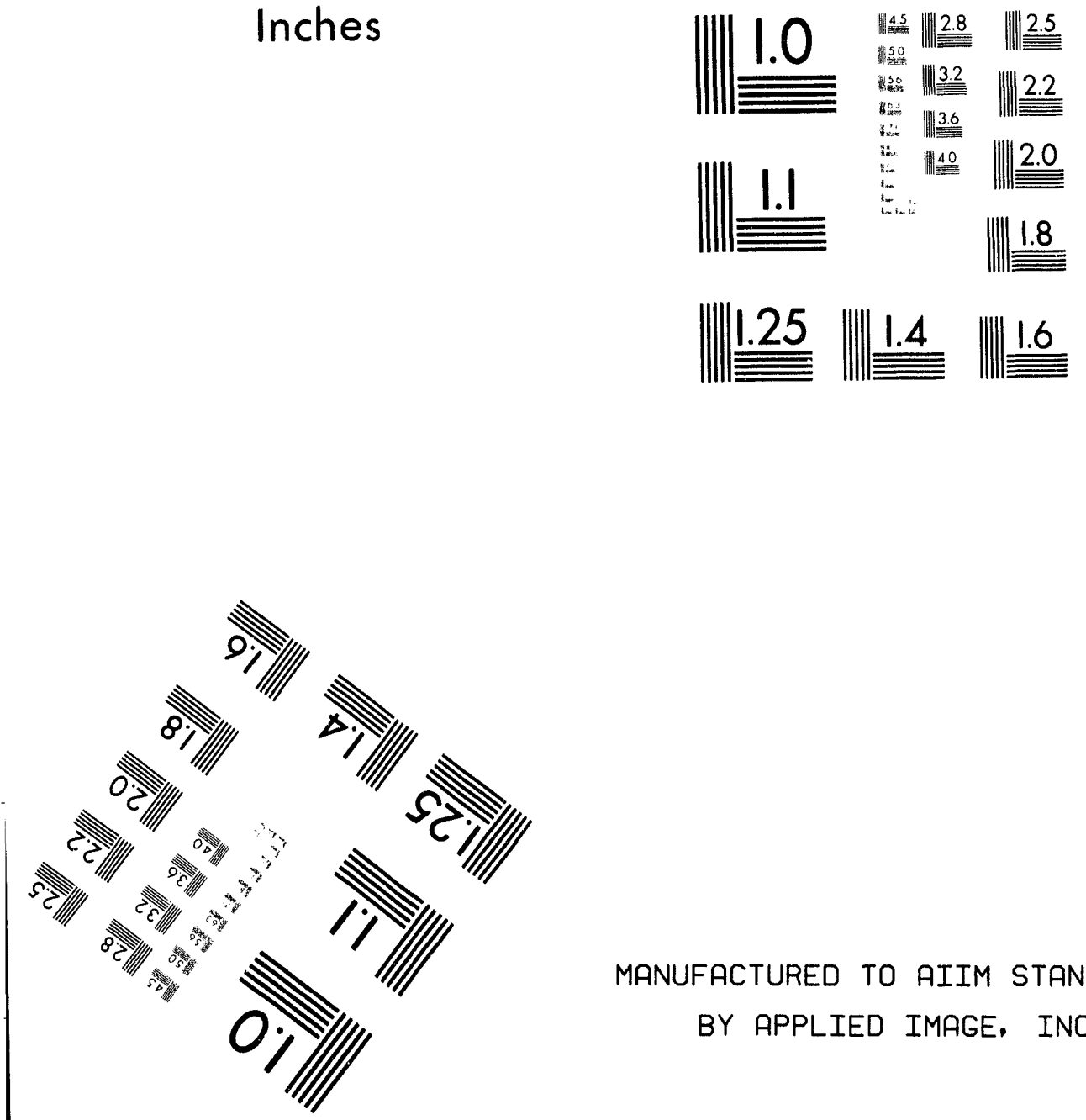

MANUFACTURED TO AIIM STANDARDS

BY APPLIED IMAGE, INC.

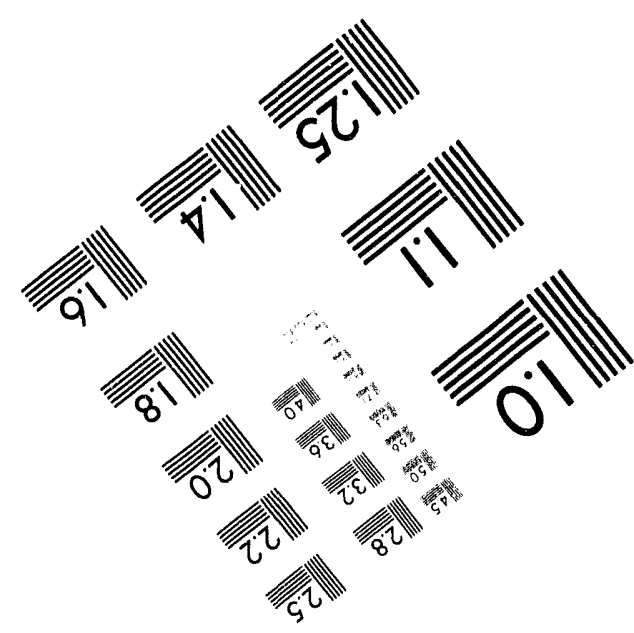



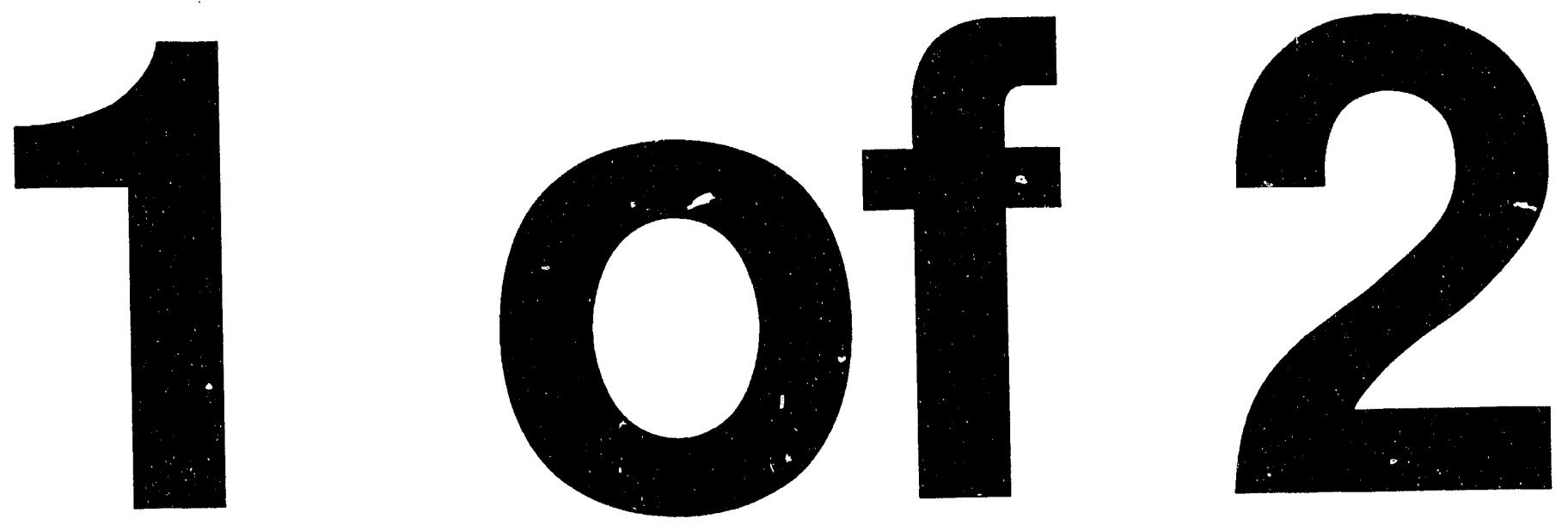
$\frac{12}{6} .94956$

UCRL-ID-116544

\section{Results from the LLNL Eulerian GRANTOUR Model for the WCRP ' 93 Workshop on the Parameterization of Sub-Grid Scale Tracer Transport}

\section{Bergmann, J. Dignon, J. Penner and J. Walton}

Global Climate Research Division

April 1994

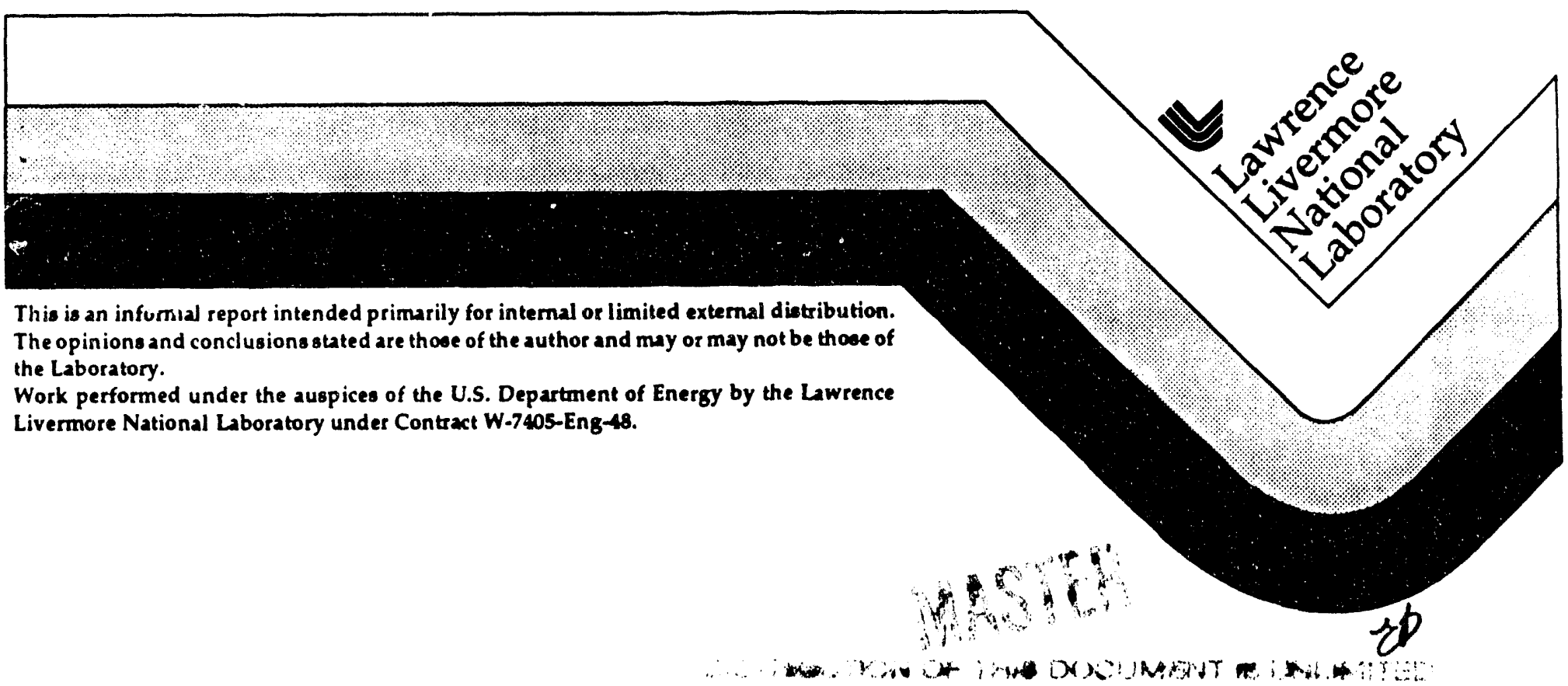




\section{DISCLAIMER}

This document was prepared as an account of work sponsored by an agency of the United States Government. Neither the United States Government nor the University of California nor any of their employees, makes any warranty, express or implied, or assumes any legal liability or responsibility for the accuracy, completeness, or usefulness of any information, apparatus, product, or process disclosed, or represents that its use would not infringe privately own rights. Reference herein to any specific commercial products, process, or service by trade name, trademark, manufacturer, or otherwise, does not necessarily constitute or imply its endorsement, recommendation, or favoring by the United States Government or the University of California. The views and opinions of authors expressed herein do not necessarily state or reflect those of the United States Government or the University of California, and shall not be used for advertising or product endorsement purposes.

This report has been reproduced directly from the best available copy.

Available to DOE and DOE contractors from the Office of Scientific and Technical Information P.O. Box 62, Oak Ridge, TN 37831

Prices available from (615) 576-8401, FTS 626-8401

Available to the public from the National Technical Information Service

U.S. Department of Commerce 5285 Port Royal Rd. Springfield, VA 22161 


\title{
Results from the LLNL Eulerian GRANTOUR Model for the WCRP '93 Workshop on the Parameterization of Sub-Grid Scale Tracer Transport
}

\author{
D. Bergmann, J. Dignon, J. Penner, and J. Walton \\ Global Climate Research Division \\ Lawrence Livermore National Laboratory
}

GRANTOUR is a 3D chemical transport model (CTM) developed at LLNL (Walton et. al., 1988) which has been applied to a diverse set of problems in the troposphere including the transport and chemistry of smoke (Ghan et al. 1988; Penner et al. 1991b), asteroid impact generated aerosols (Covey et al., 1990) sulfate aerosols (Erickson et al., 1991; Penner et al., 1993a), NOx (Penner et al. 1991a), ozone (Atherton 1993; Atherton et al. 1993; Penner et al. 1993a), CFC-11 (Penner, 1990), ${ }^{7} \mathrm{Be}$ (in progress), and ${ }^{222} \mathrm{Rn}$ with decay to $210 \mathrm{~Pb}$ (Dignon et al., 1993). GRANTOUR uses fields supplied by a GCM (General Circulation Model) and calculates the advection of inert or chemically reactive trace species in a Lagrangian scheme. The model can be run either as an off-line model which uses fields precalculated by a GCM or in a coupled mode where the results from the CTM are used in the radiation calculations of the GCM. The atmosphere is divided into "parcels" of equal mass and the center of mass of each of these parcels is accurately advected in a Lagrangian manner with little or no numerical diffusion. The advantage of this approach is the accuracy of the advection, while the disadvantages of this scheme are the difficulties in resolving the stratosphere and accurately inferring fluxes from the movements of the center of masses of the parcels.

An Eulerian option has recently bien inplemented in GRANTOUR which uses a second order Van Leer scheme to transport inert or reactive trace species through a fixed three dimensional grid. Although this ne $v$ model introduces a small amount of numerical diffusion which the Lagrangian approach did not have, it provides the potential to accurately resolve the stratosphere and to diagnose important quantities such as fluxes across the equator or the tropopause.

Both sets of GRANTOUR radon calculations performed for the WCRP workshop used time averaged 12 hour fields from the CCM1 model in an uncoupled mode. The Lagrangian results were obtained from a resolution of 50,000 parcels, while the Eulerian results used the CCM1 resolution of approximately $7.5^{\circ}$ (longitude) by $4.5^{\circ}$ (latitude) and 13 sigma levels.

Mixing is calculated on a fixed grid for both the Lagrangian and Eulerian versions, and includes both convection and diffusion. Convection is identical in each version and the convective mass flux is diagnosed from the CCM1 model (Penner er al., 1991). Large scale diffusion uses a set of fixed eddy coefficients in the stratosphere $(\sigma<0.2)$ and a second set in the troposphere. A horizontal diffusion coefficient of $1 \times 10^{5} \mathrm{~cm}^{2} / \mathrm{sec}$ was used in the stratosphere while a value of $1 \times 10^{8} \mathrm{~cm}^{2} / \mathrm{sec}$ was used in the troposphere. Vertical diffusion coefficients of $1 \times 10^{2} \mathrm{~cm}^{2} / \mathrm{sec}$ in the stratosphere and $1 \times 10^{4} \mathrm{~cm}^{2} / \mathrm{sec}$ in the troposphere were used. An additional mixing term is used in the Lagrangian version to simulate inter-parcel mixing.

The chemistry model for these radon calculations is a simple exponential decay of $222 \mathrm{Rn}$ into $210 \mathrm{~Pb}$ with an e-folding lifetime of 5.5 days. The $210 \mathrm{~Pb}$ produced from this decay is tracked as a second species. 
Deposition models for both wet and dry scavenging of $210 \mathrm{~Pb}$ are included in both versions. Wet scavenging is proportional to the precipitation rate obtained from the CCM1, while dry scavenging uses a fixed deposition velocity of $0.1 \mathrm{~cm} / \mathrm{sec}$.

Some minor differences exist between the radon problem specifications and their implementation in the two GRANTOUR versions. For the natural radon calculation (case A) the Lagrangian version injected radon into all parcels located between sigma values of 0.9 and 1.0, while the Eulerian version injected into grid boxes between sigma values of 0.926 and 1.0. The height of the aircraft and lightning injections is between 200 and 400 $\mathrm{mb}$ in the Lagrangian version. In the Eulerian version we injected the material between sigma coordinates of 0.165 and 0.355 . The precise horizontal locations of the aircraft and lightning sources differs very slightly between the two versions and is explained in the data package submitted to the workshop. Output from the Lagrangian version labeled mixed layer is actually at $977 \mathrm{mb}$, while in the Eulerian version it is at $\sigma=0.965$.

References:

Atherton, C.S., 1993: Ph.D. Dissertation, University of California, Davis.

Atherton, C.S., J. Dignon, J. Penner, S. Sillman, and J. Walton, 1993: Man's impact on tropospheric $\mathrm{O}_{3}$ and $\mathrm{OH}$ : Global model results, presented at the IAMAP-IAHS International Scientific Meeting, session on Major Advances of Atmospheric Chemistry over the Past Decade and Their Impact on Public Welfare, Yokohama, Japan, July 11-23,.1993.

Covey, C., S. J. Ghan, J. J. Walton and P. R. Weissman, "Global Environmental Effects of Impact-Generated Aerosols: Results From a General Circulation Model," Global Catastrophes in Earth History: An Interdisciplinary Conference on Impacts, Volcanism and Mass Mortality, V.L. Sharpton and P.D. Ward (Eds.), pp. 263-270, Geological Society of America, Boulder, Colo., 1990.

Dignon, J.E. Penner, and J. J. Walton, , "Global Simulation of ${ }^{222} \mathrm{Rn}$ and $210 \mathrm{~Pb}$ in a 3Dimensional Lagrangian Tracer Model," draft manuscript, in preparation, 1993.

Erickson III, D.J., J.J. Walton, S.J. Ghan, and J.E. Penner, 1991: Three-dimensional modeling of the global atmospheric sulfur cycle: A first step, Atmos. Environ., 25A, 2513-2520.

Ghan, S. J., M. C. MacCracken, and J. J. Walton, "Climatic Response to Large Atmospheric Smoke Injections: Sensitivity Studies with a Tropospheric General Circulation Model," J. Geophys. Res., 93, 8315-8337, 1988.

Penner, J.E., 1990: Special Numerical Experiment: Simulation of $\mathrm{CFCl}_{3}$ as a Test for 3-D Atmospheric Models, WCRP Workshop on Long-Range Transport of Trace Gases M. Prather (Ed.) Bermuda, December 10-14 1990.

Penner, J.E., C.S. Atherton, J. Dignon, S.J. Ghan, J.J. Walton, and S. Hameed, 1991a: Tropospheric nitrogen: A three-dimer-ional study of sources, distribution, and deposition, J. of Geophys. Res., 96, 959-990. 
Penner, J.E., S.J. Ghan, and J.J. Walton, 1991b: The role of biomass burning in the budget and cycle of carbonaceous soot aerosols and their climate impact, in Global Biomass Burning, ed. by J. Levine, MIT press, Cambridge, NA, 387-393.

Penner, J.E., C.A. Atherton, and T. Graedel, 1993a: Global emissions and models of photochemically active compounds, to be published in Global AtmosphericBiospheric Chemistry: The First IGAC Scientific Conference, OHOLO Conference Series Books, Plenum Publishing, N.Y. (Also UCRL-JC-112273.)

Penner, J.E., H. Eddleman and T. Novakov, 1993b: Towards the development of a global inventory of black carbon emissions, Atmos. Environ., 27A, 1277-1295.

Walton, J. J., M. C. MacCracken and S. J. Ghan, "A Global-Scale Lagrangian Trace Species Model of Transport, Transformation, and Removal Processes," J. Geophys. Res., 93, 8339-8354, 1988. 
Case A: Surface (natural radon) 
RADON-222 Mixing Ratio
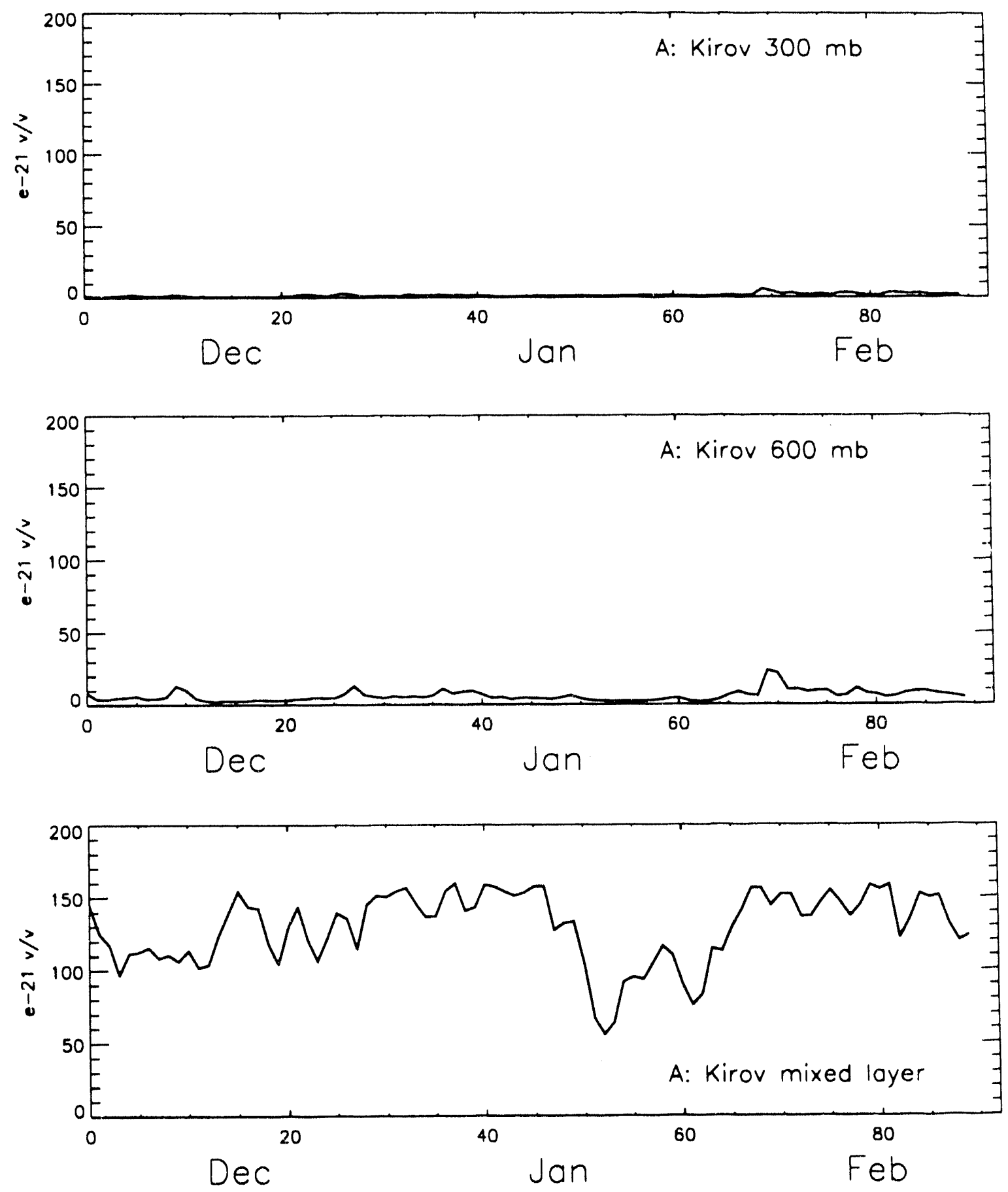
EULERIAN GRANTOUR-LLNL

Dec-Jan-Feb

RADON-222 Mixing Ratio
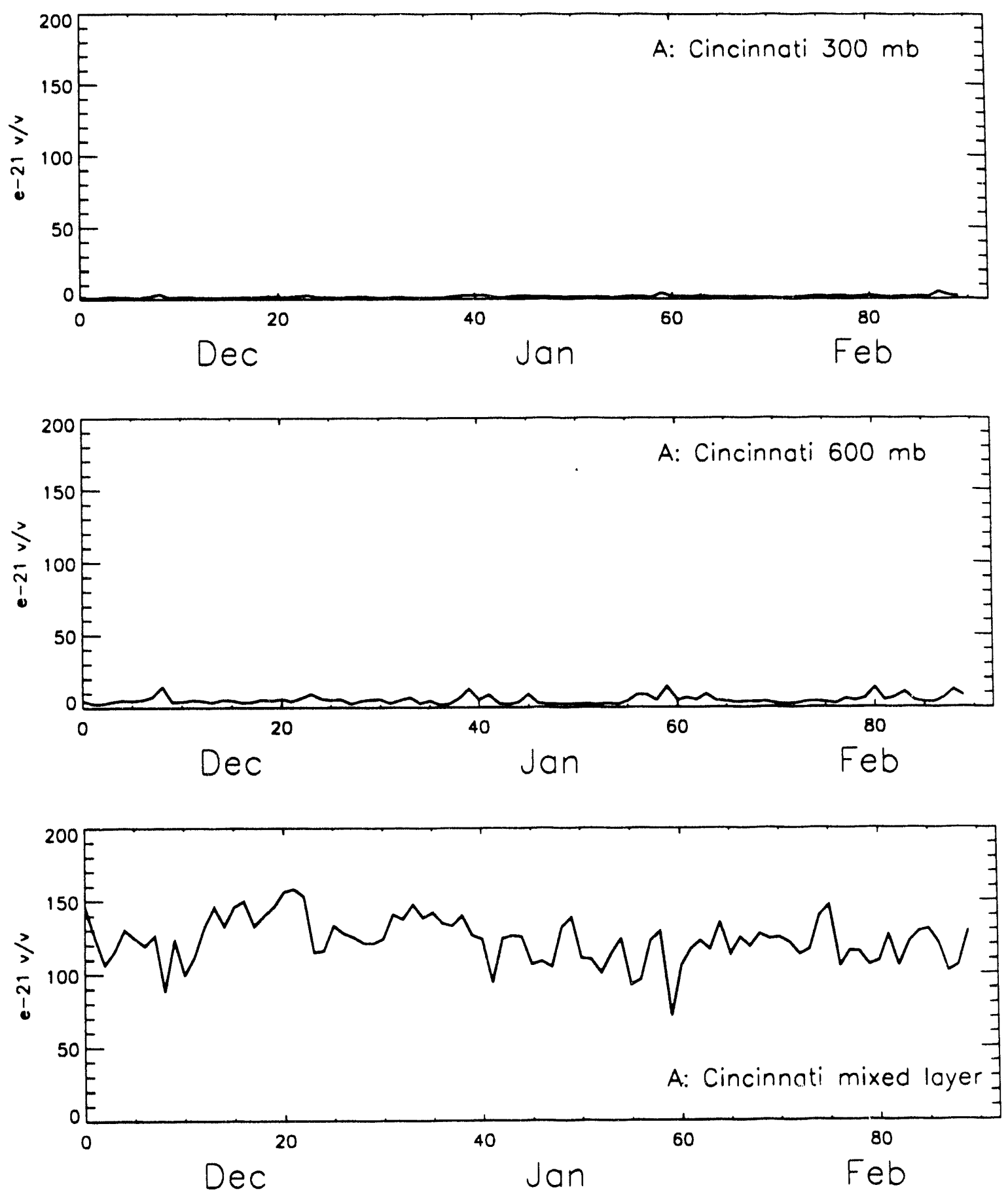

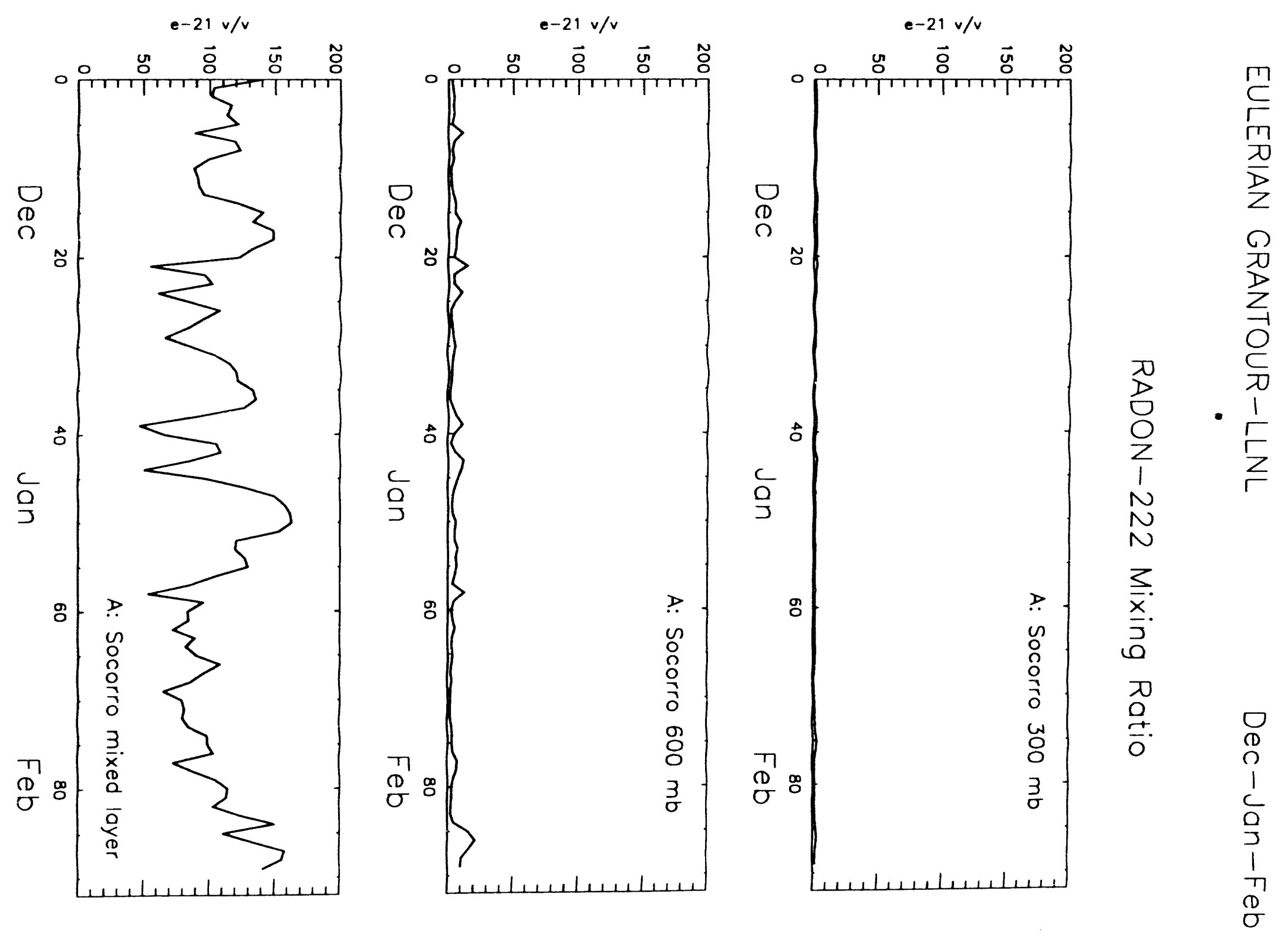
EULERIAN GRANTOUR-LLNL

Dec-Jan-Feb

RADON-222 Mixing Ratio
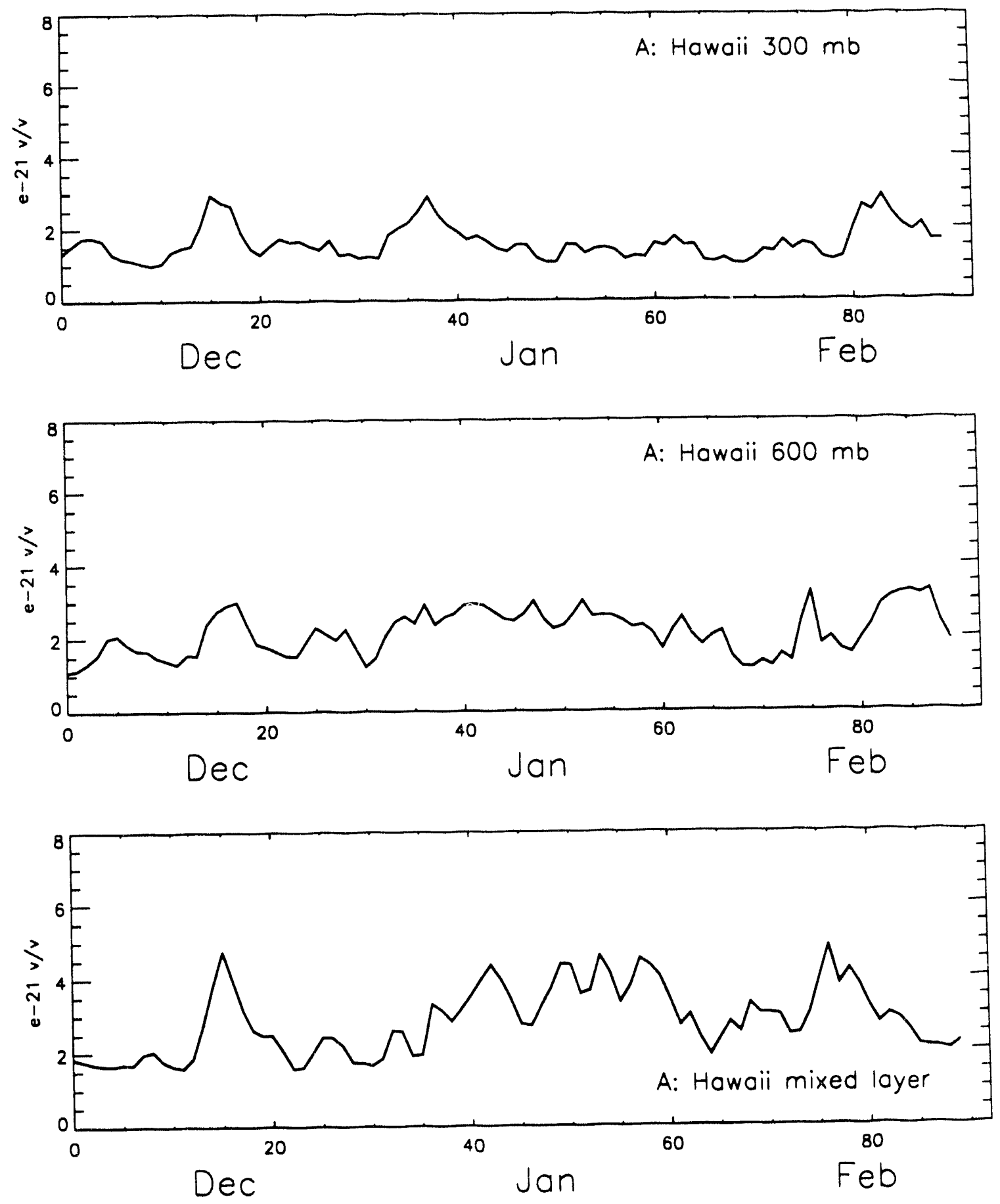
RADON-222 Mixing Ratio
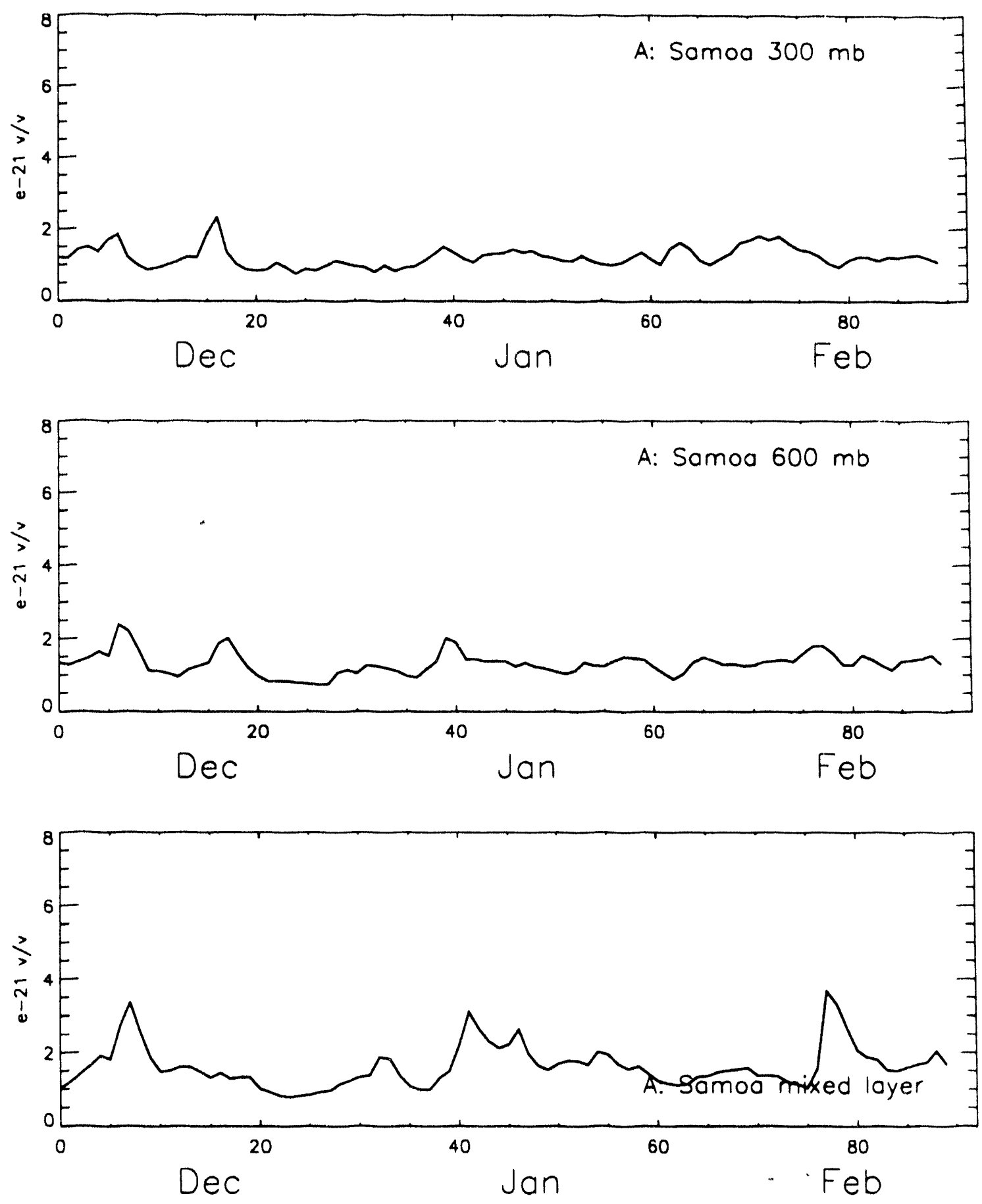
RACON-222 Mixing Ratio
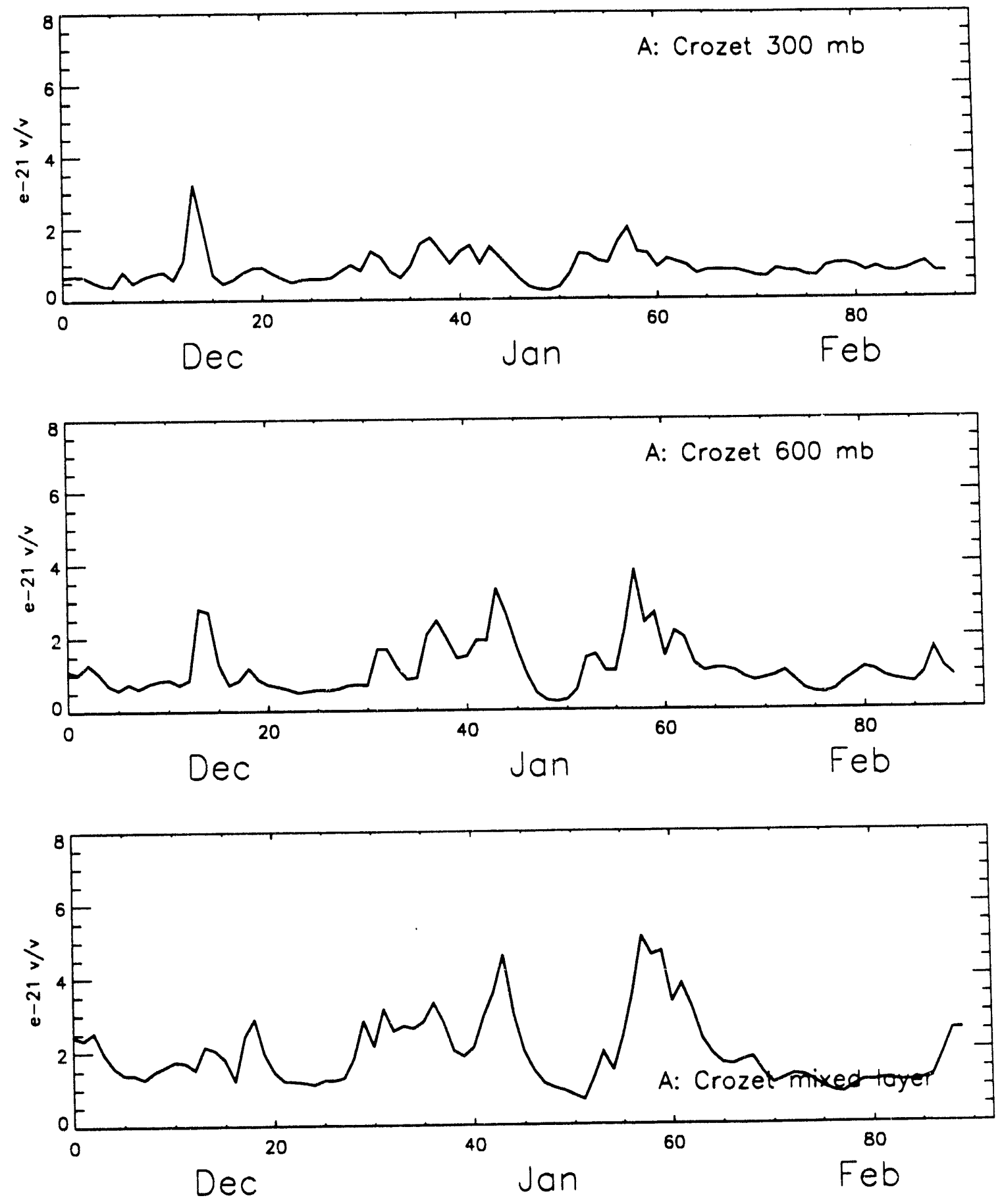


$$
\text { RADON-222 Mixing Ratio }
$$
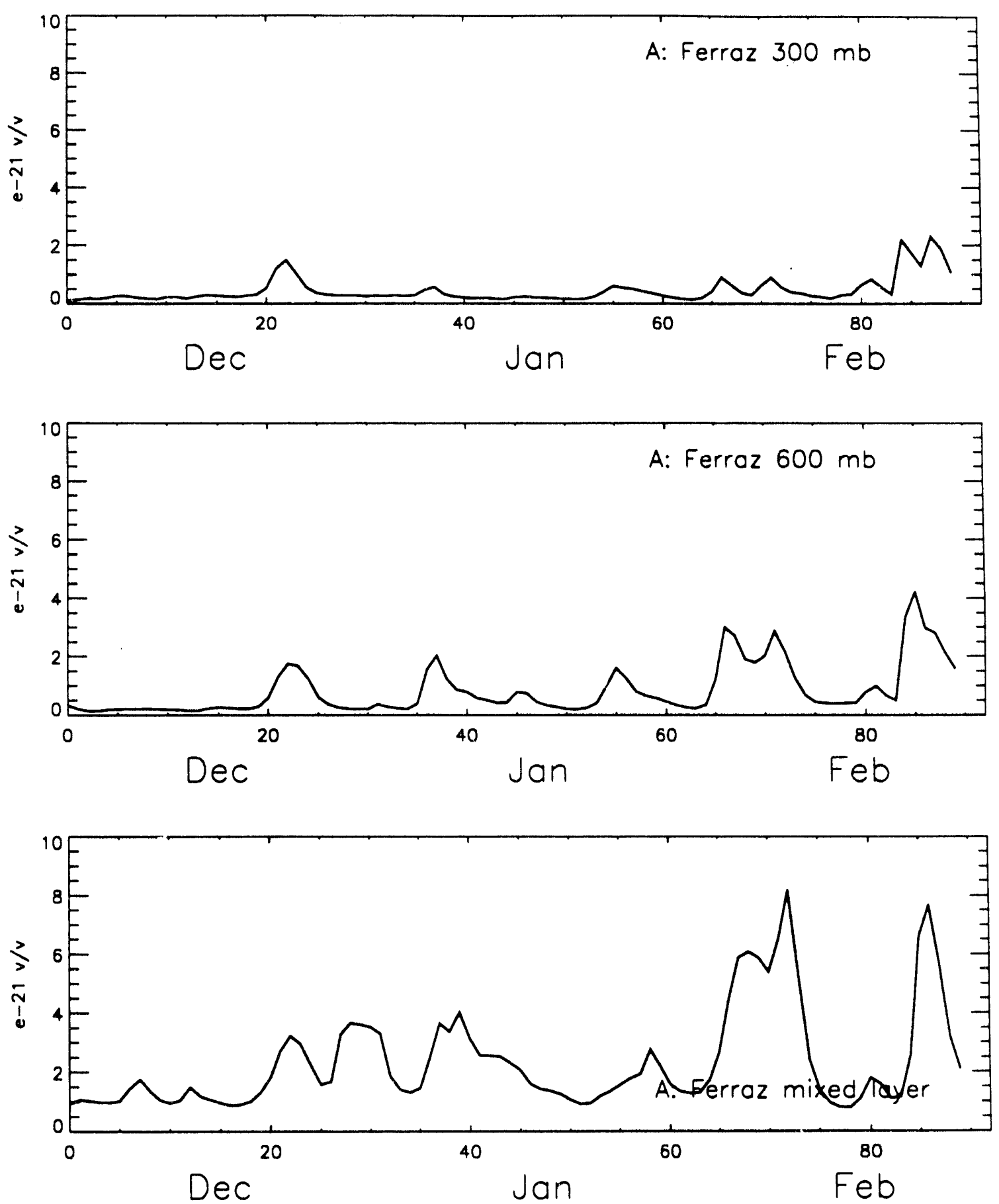


\begin{tabular}{|c|c|c|c|c|c|c|c|c|c|}
\hline \multicolumn{10}{|c|}{ EULERIAN GRANTOUR-LLNL Case A: Time serles statistics (E-21 v/v) Dec-Jan-Feb } \\
\hline site & level & mean & stdv & $\operatorname{var}^{\star} \mathrm{E}-42$ & $\min$ & $1 / 4$ & median & $3 / 4$ & $\max$ \\
\hline Kirov & $\begin{array}{c}300 \mathrm{mb} \\
600 \mathrm{mb} \\
\text { mixed } \\
\text { surface }\end{array}$ & $\begin{array}{r}1.51 \\
5.94 \\
129.40 \\
138.03 \\
\end{array}$ & \begin{tabular}{r|}
0.83 \\
3.62 \\
24.38 \\
24.03 \\
\end{tabular} & $\begin{array}{r}0.69 \\
13.09 \\
594.54 \\
577.39 \\
\end{array}$ & \begin{tabular}{r|}
0.68 \\
1.87 \\
55.92 \\
64.76 \\
\end{tabular} & $\begin{array}{r}0.97 \\
3.70 \\
113.13 \\
122.23 \\
\end{array}$ & $\begin{array}{r}1.32 \\
5.06 \\
136.82 \\
144.85 \\
\end{array}$ & $\begin{array}{r}1.72 \\
7.19 \\
151.27 \\
158.70 \\
\end{array}$ & $\begin{array}{r}5.67 \\
23.42 \\
159.75 \\
169.22 \\
\end{array}$ \\
\hline Cincinnati & $\begin{array}{c}300 \mathrm{mb} \\
600 \mathrm{mb} \\
\text { mixed } \\
\text { surface }\end{array}$ & $\begin{array}{r}1.68 \\
5.12 \\
123.07 \\
133.13 \\
\end{array}$ & $\begin{array}{r}0.67 \\
2.78 \\
15.90 \\
16.36 \\
\end{array}$ & $\begin{array}{r}0.45 \\
7.74 \\
252.80 \\
267.65 \\
\end{array}$ & $\begin{array}{r}0.81 \\
1.76 \\
70.79 \\
76.59 \\
\end{array}$ & $\begin{array}{r}1.22 \\
3.26 \\
113.34 \\
123.82 \\
\end{array}$ & $\begin{array}{r}1.56 \\
4.69 \\
124.20 \\
132.99 \\
\end{array}$ & $\begin{array}{r}1.83 \\
6.04 \\
132.77 \\
143.86 \\
\end{array}$ & $\begin{array}{r}5.28 \\
14.26 \\
158.20 \\
167.83 \\
\end{array}$ \\
\hline Socorro & $\begin{array}{c}300 \mathrm{mb} \\
600 \mathrm{mb} \\
\text { mixed } \\
\text { surface }\end{array}$ & $\begin{array}{r}1.61 \\
5.27 \\
106.51 \\
118.90 \\
\end{array}$ & $\begin{array}{r}0.48 \\
3.60 \\
27.29 \\
25.83 \\
\end{array}$ & $\begin{array}{r}0.23 \\
12.96 \\
744.66 \\
667.17 \\
\end{array}$ & $\begin{array}{r}0.86 \\
1.72 \\
46.87 \\
64.63 \\
\end{array}$ & $\begin{array}{r}1.24 \\
2.84 \\
86.56 \\
100.76 \\
\end{array}$ & $\begin{array}{r}1.49 \\
4.06 \\
104.50 \\
115.38 \\
\end{array}$ & $\begin{array}{r}1.90 \\
5.87 \\
123.68 \\
137.83 \\
\end{array}$ & $\begin{array}{r}3.05 \\
21.10 \\
162.91 \\
173.79 \\
\end{array}$ \\
\hline Hawaii & $\begin{array}{c}300 \mathrm{mb} \\
600 \mathrm{mb} \\
\text { mixed } \\
\text { surface }\end{array}$ & $\begin{array}{r}1.60 \\
2.15 \\
2.84 \\
2.90 \\
\end{array}$ & $\begin{array}{l}0.47 \\
0.59 \\
0.91 \\
0.93 \\
\end{array}$ & $\begin{array}{l}0.22 \\
0.35 \\
0.83 \\
0.87 \\
\end{array}$ & $\begin{array}{l}0.99 \\
1.10 \\
1.57 \\
1.63 \\
\end{array}$ & $\begin{array}{r}1.23 \\
1.65 \\
2.02 \\
2.03 \\
\end{array}$ & $\begin{array}{r}1.52 \\
2.20 \\
2.75 \\
2.75 \\
\end{array}$ & $\begin{array}{l}1.77 \\
2.59 \\
3.55 \\
3.62 \\
\end{array}$ & $\begin{array}{l}2.95 \\
3.26 \\
4.86 \\
4.85 \\
\end{array}$ \\
\hline Samoa & $\begin{array}{c}300 \mathrm{mb} \\
600 \mathrm{mb} \\
\text { mixed } \\
\text { surface } \\
\end{array}$ & \begin{tabular}{l|}
1.22 \\
1.31 \\
1.62 \\
1.67 \\
\end{tabular} & $\begin{array}{l}0.28 \\
0.31 \\
0.58 \\
0.57 \\
\end{array}$ & $\begin{array}{l}0.08 \\
0.09 \\
0.33 \\
0.33 \\
\end{array}$ & \begin{tabular}{l|}
0.76 \\
0.73 \\
0.79 \\
0.84 \\
\end{tabular} & $\begin{array}{l}1.02 \\
1.12 \\
1.29 \\
1.30 \\
\end{array}$ & $\begin{array}{l}1.20 \\
1.30 \\
1.52 \\
1.56 \\
\end{array}$ & $\begin{array}{l}1.35 \\
1.43 \\
1.83 \\
1.91 \\
\end{array}$ & $\begin{array}{l}2.33 \\
2.38 \\
3.68 \\
3.56 \\
\end{array}$ \\
\hline Crozet & $\begin{array}{c}300 \mathrm{mb} \\
600 \mathrm{mb} \\
\text { mixed } \\
\text { surface }\end{array}$ & $\begin{array}{l}0.89 \\
1.16 \\
1.95 \\
1.98 \\
\end{array}$ & $\begin{array}{l}0.43 \\
0.69 \\
0.95 \\
0.96 \\
\end{array}$ & $\begin{array}{l}0.18 \\
0.48 \\
0.91 \\
0.92 \\
\end{array}$ & $\begin{array}{l}0.24 \\
0.22 \\
0.67 \\
0.74 \\
\end{array}$ & $\begin{array}{l}0.63 \\
0.72 \\
1.21 \\
1.22 \\
\end{array}$ & $\begin{array}{l}0.79 \\
0.92 \\
1.73 \\
1.77 \\
\end{array}$ & $\begin{array}{l}1.00 \\
1.42 \\
2.52 \\
2.55 \\
\end{array}$ & $\begin{array}{l}3.22 \\
3.81 \\
5.11 \\
5.18 \\
\end{array}$ \\
\hline Ferraz & $\begin{array}{c}300 \mathrm{mb} \\
600 \mathrm{mb} \\
\text { mixed } \\
\text { surface }\end{array}$ & \begin{tabular}{l|}
0.45 \\
0.84 \\
2.34 \\
2.28 \\
\end{tabular} & \begin{tabular}{l|}
0.44 \\
0.87 \\
1.68 \\
1.56 \\
\end{tabular} & $\begin{array}{l}0.19 \\
0.76 \\
2.81 \\
2.44 \\
\end{array}$ & $\begin{array}{l}0.11 \\
0.14 \\
0.80 \\
0.82 \\
\end{array}$ & $\begin{array}{l}0.21 \\
0.23 \\
1.16 \\
1.16 \\
\end{array}$ & $\begin{array}{l}0.28 \\
0.43 \\
1.67 \\
1.67 \\
\end{array}$ & \begin{tabular}{l|}
0.50 \\
1.23 \\
2.96 \\
2.93 \\
\end{tabular} & $\begin{array}{l}2.30 \\
4.22 \\
8.19 \\
7.67 \\
\end{array}$ \\
\hline
\end{tabular}


EULERIAN GRANTOUR-LLNL DJF

A: Mixed Layer

RADON-222 Mixing Ratio [*e21 v/v]

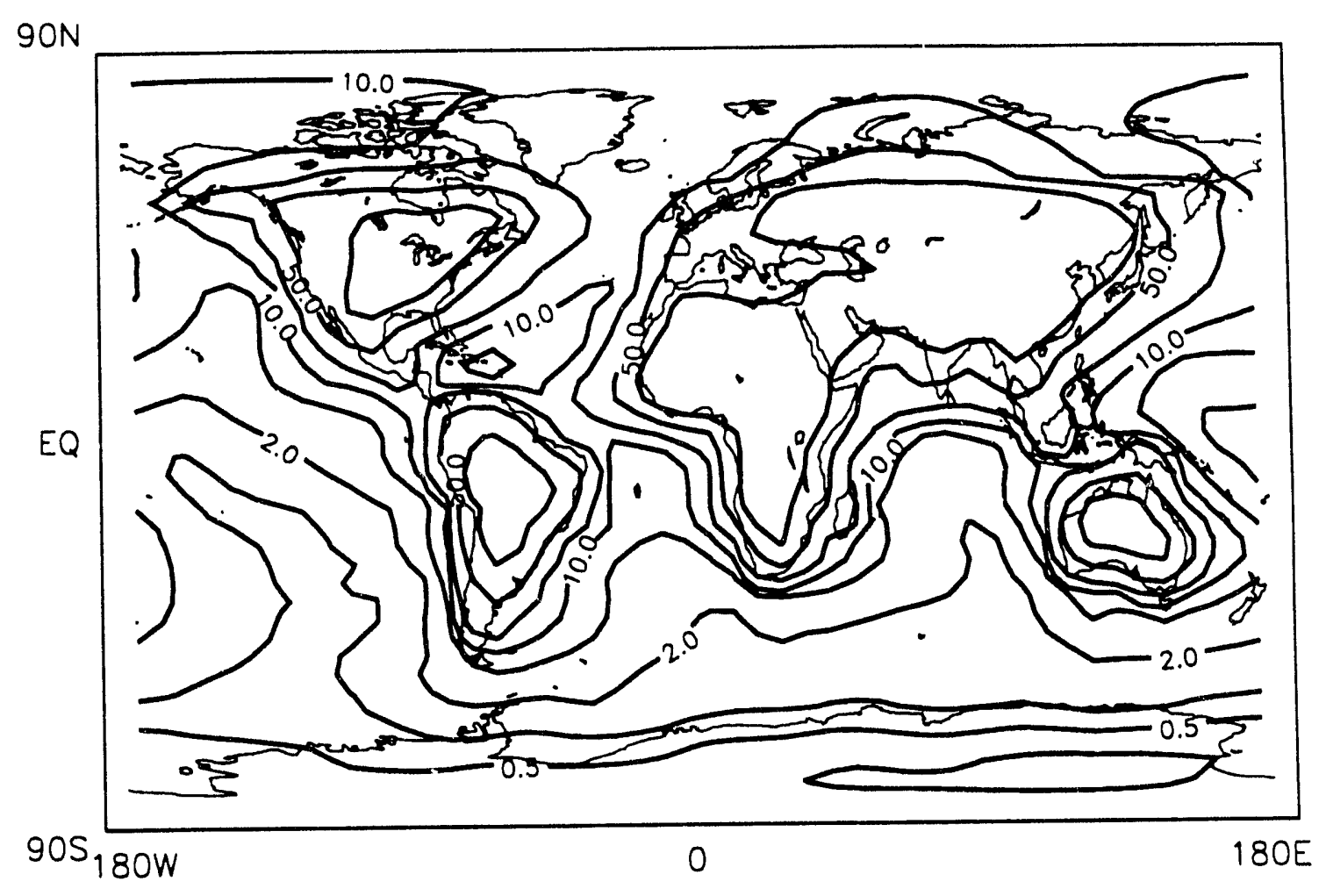


EULERIAN GRANTOUR-LLNL

\section{A: $600 \mathrm{mb}$}

$$
\text { RADON-222 Mixing Ratio [*e21 v/v] }
$$

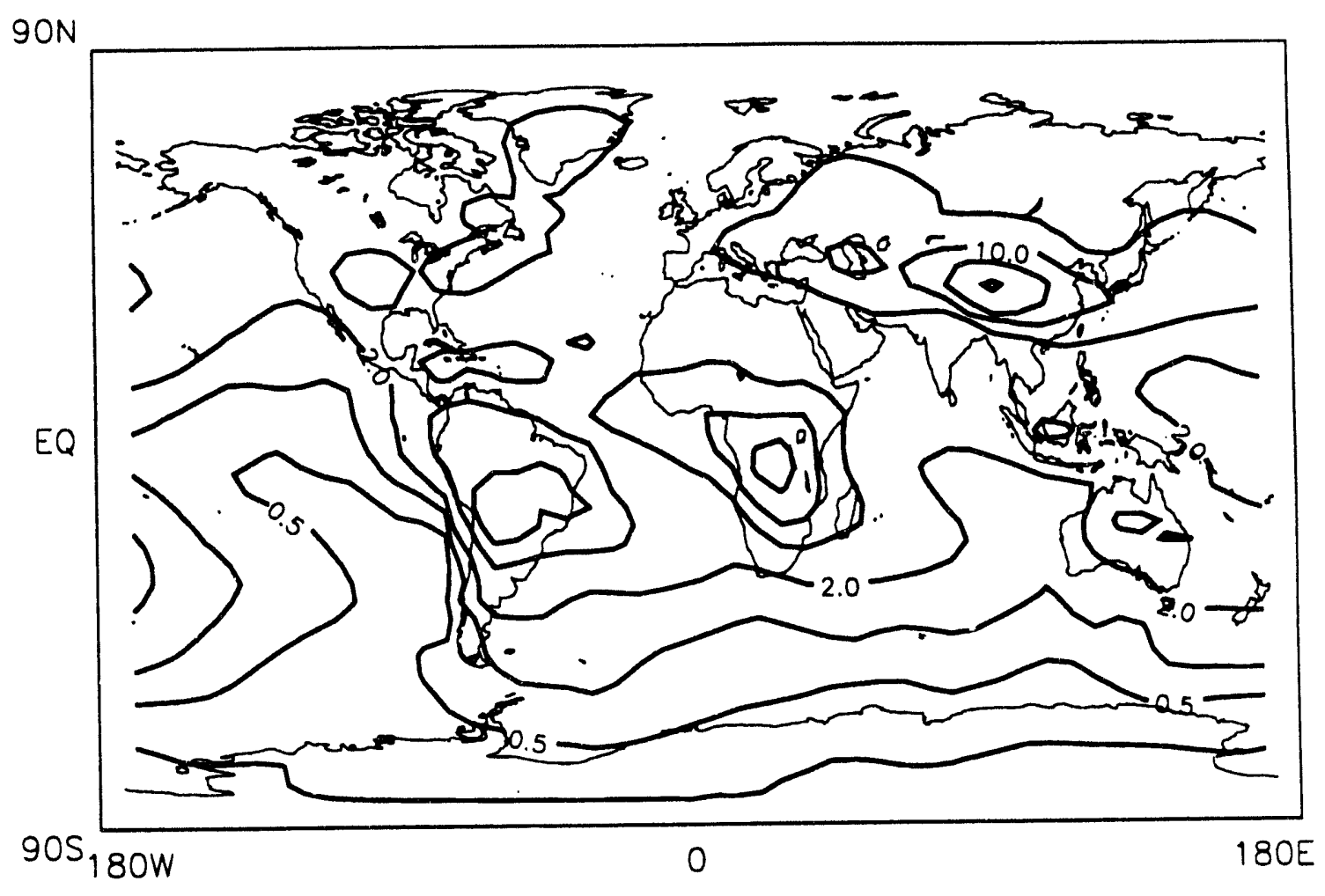


EULERIAN GRANTOUR-LLNL DJF

A: $300 \mathrm{mb}$

RADON-222 Mixing Ratio [*e21 v/v]

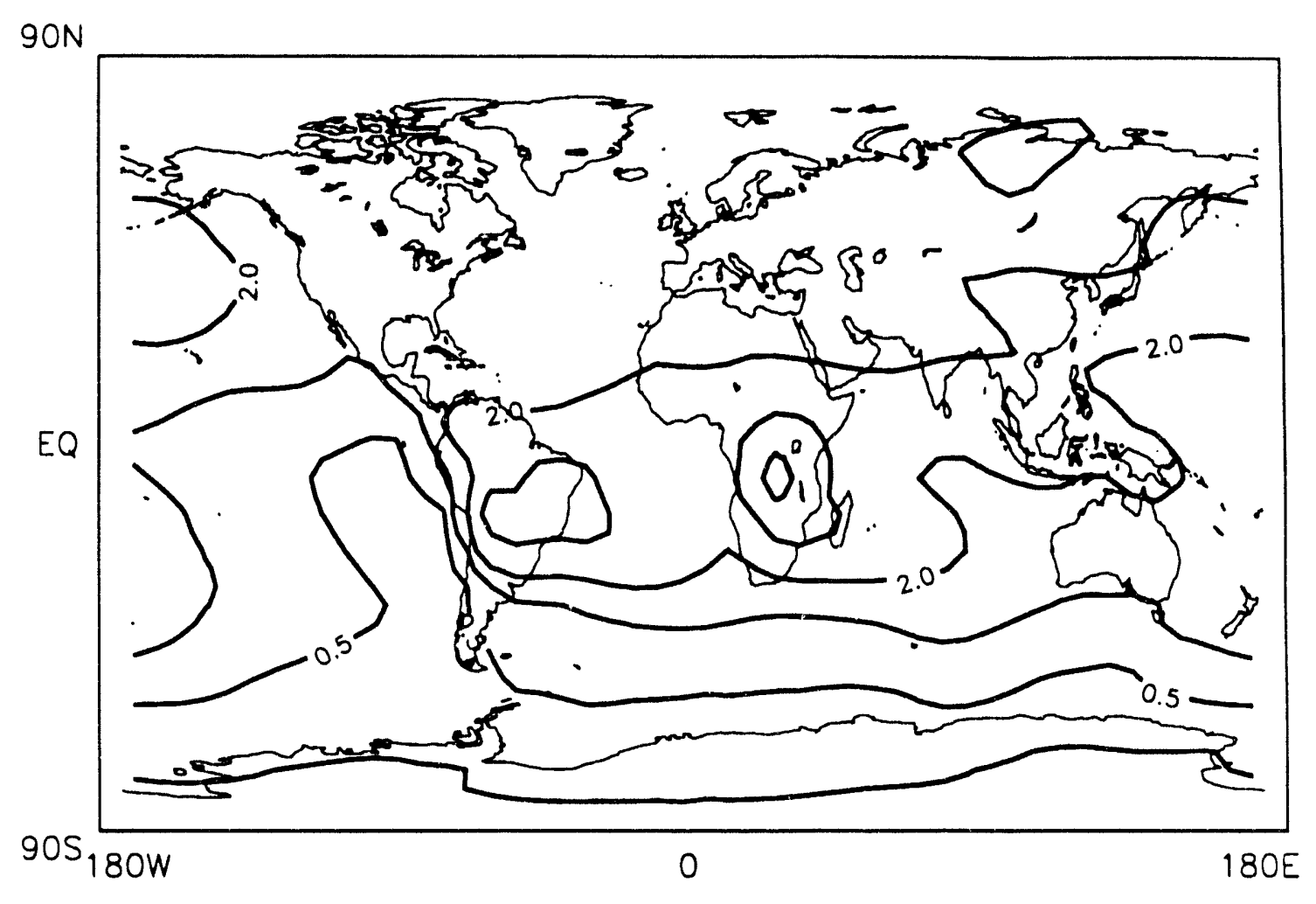


EULERIAN GRANTOUR-LLNL

DJF

A: Zonal Avg

RADON-222 Mixing Rotio [*e21 v/v]

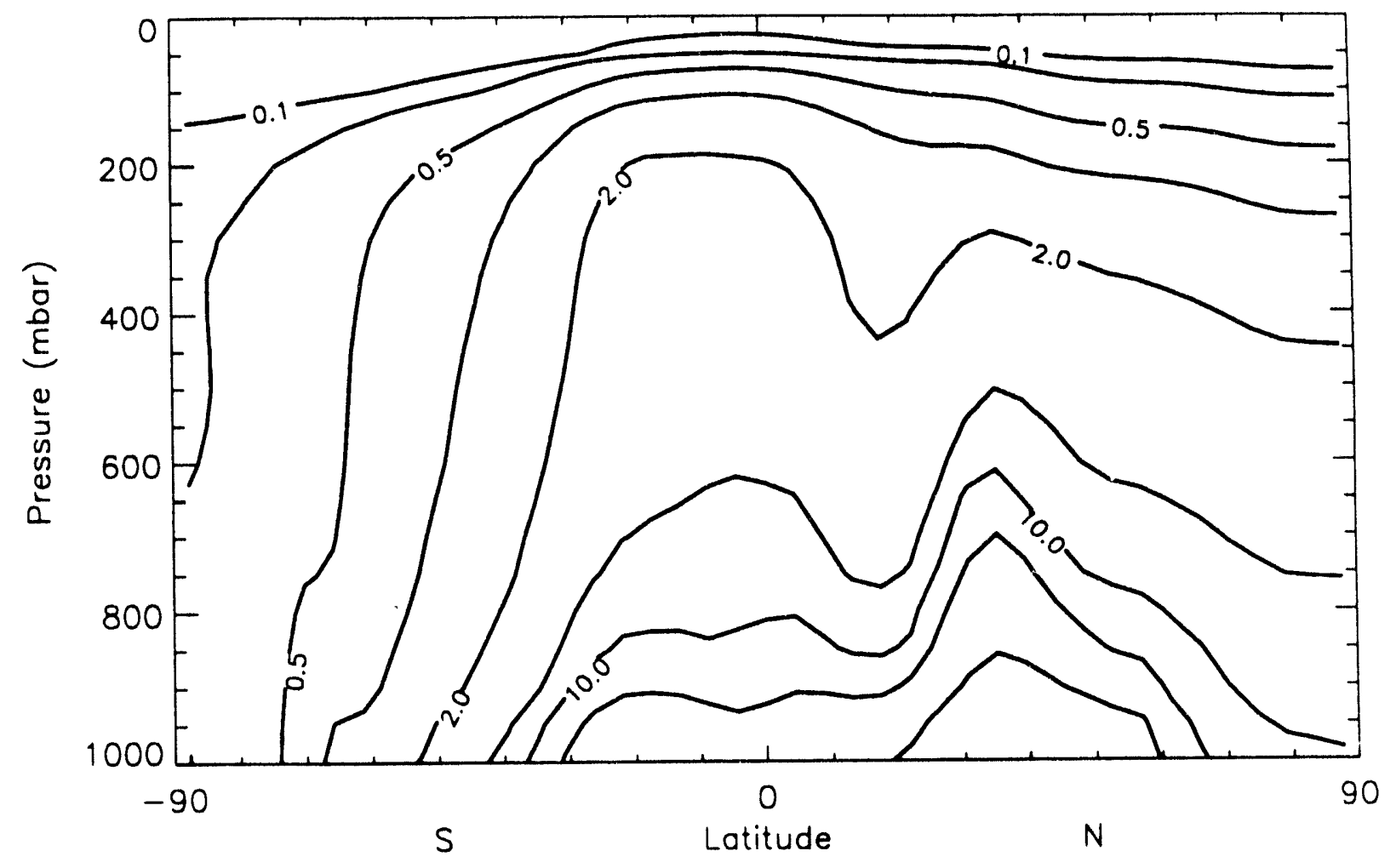


EULERIAN GRANTOUR-LLNL

A: OEW

RADON-222 Mixing Rotio [*e21 v/v]

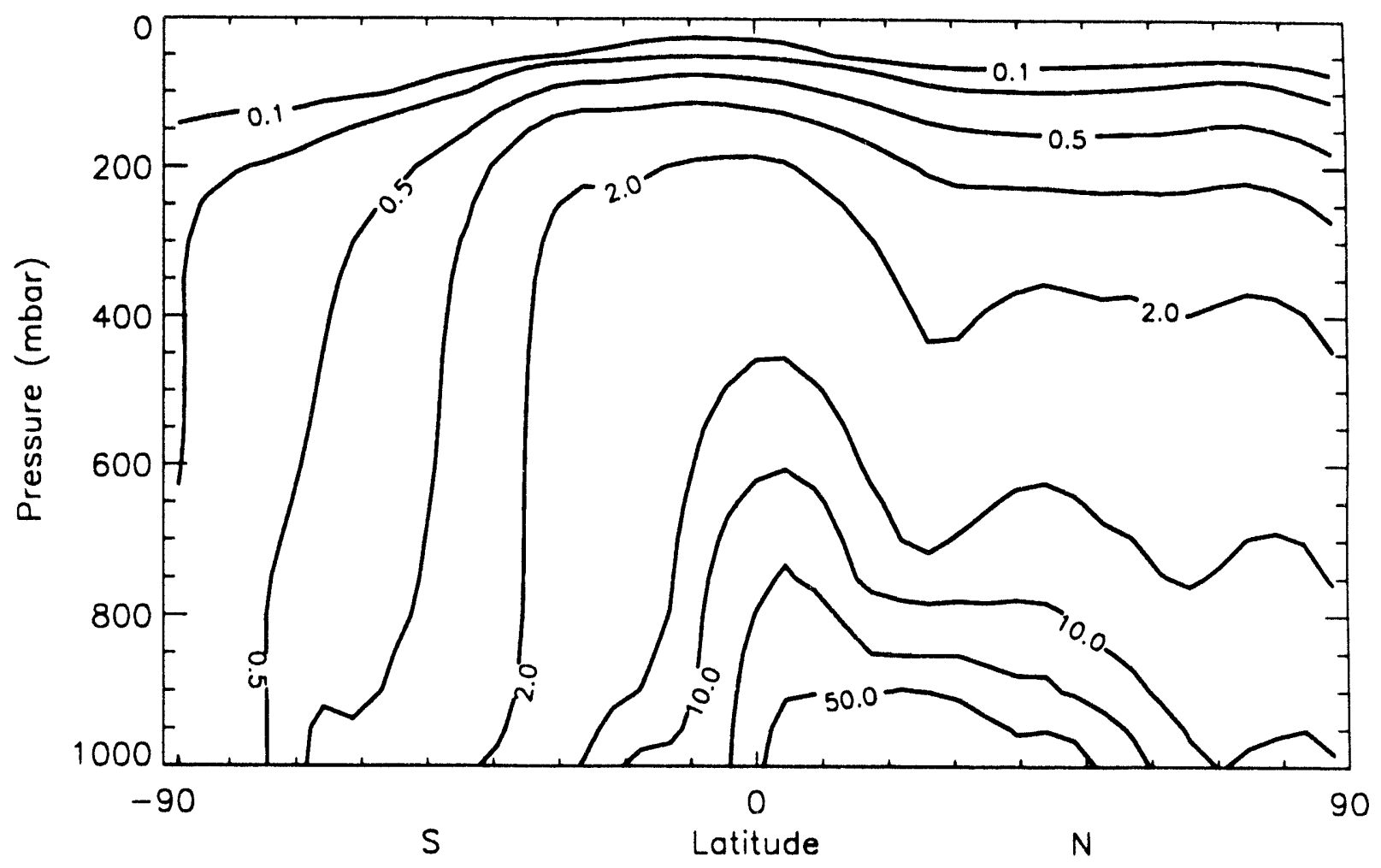




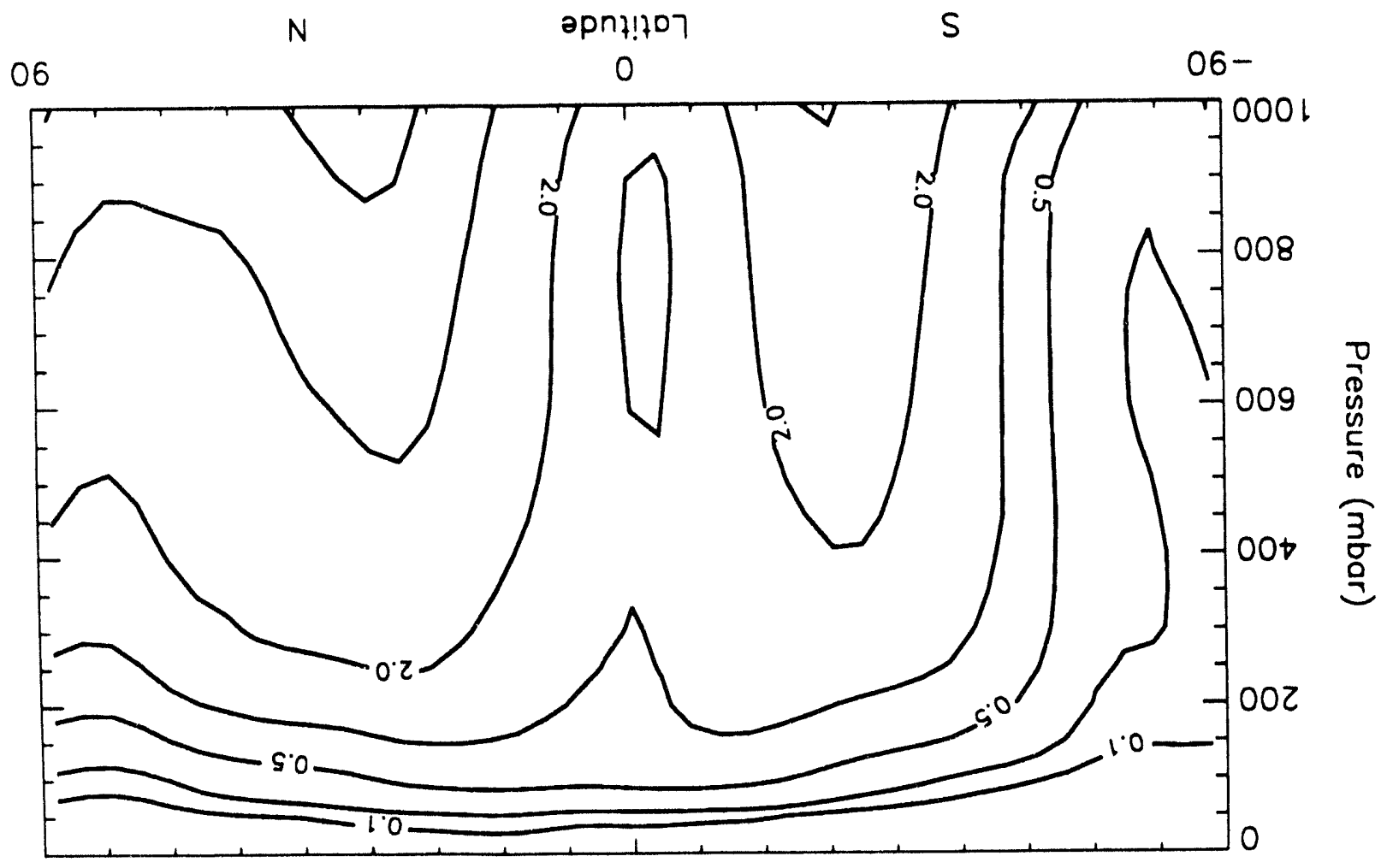

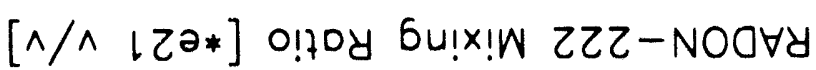

$M 3081: \forall$

$\exists \Gamma O$

$7 N\urcorner 7-y \cap O \perp N \forall y O N \forall \forall \forall \exists 7 \cap \exists$ 
EULERIAN GRANTOUR-LLNL

A: Zonal Avg

LEAD-210 Mixing Ratio [*e21 v/v]

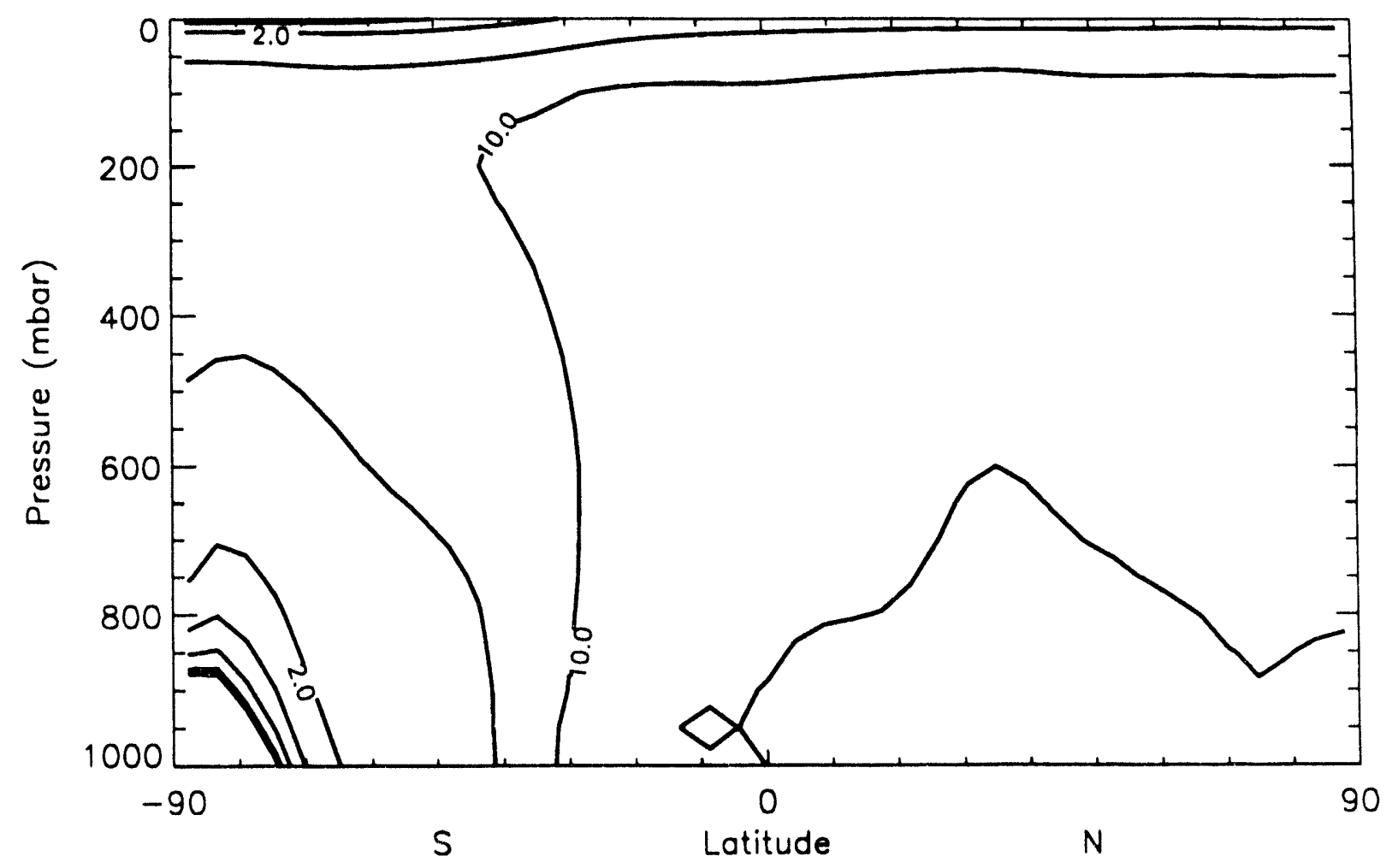


EULERIAN GRANTOUR-LLNL

DJF

A: OEW

LEAD-210 Mixing Ratio [*e21 v/v]

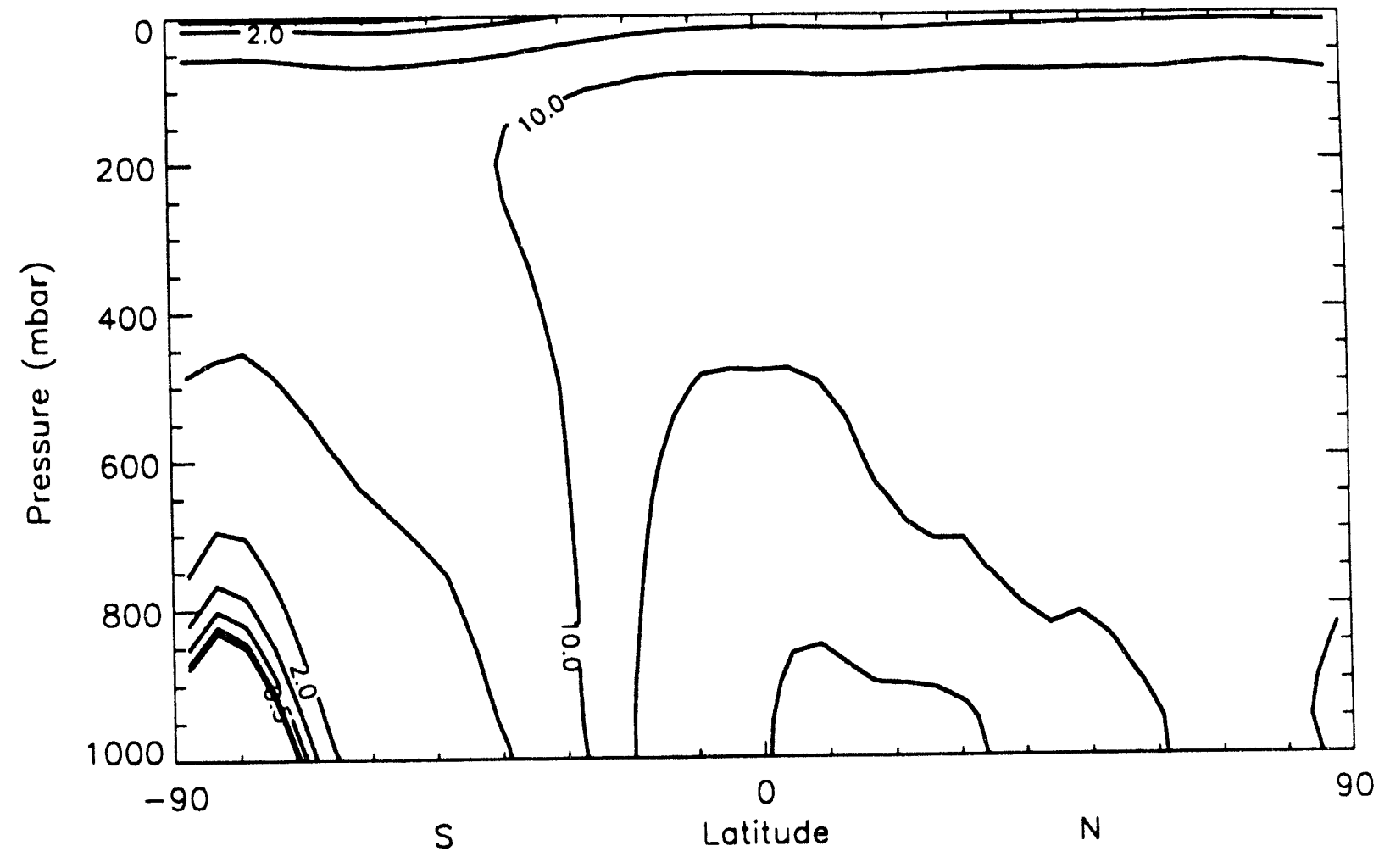


EULERIAN GRANTOUR-LLNL

\section{A: $180 E W$}

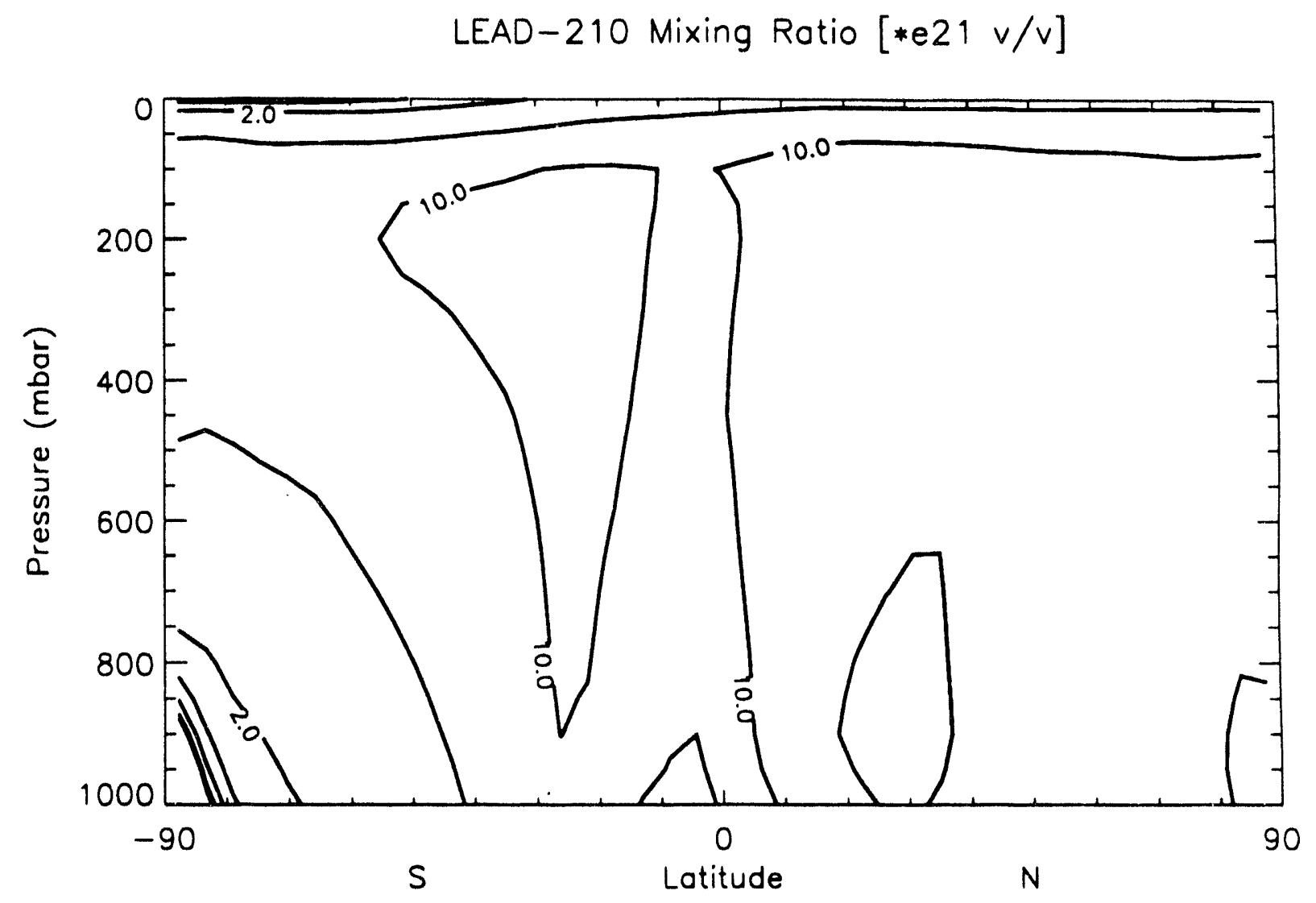


EULERIAN GRANTOUR-LLNL

DJF

A: Mixed Layer

LEAD-210 Mixing Ratio [*e21 v/v]

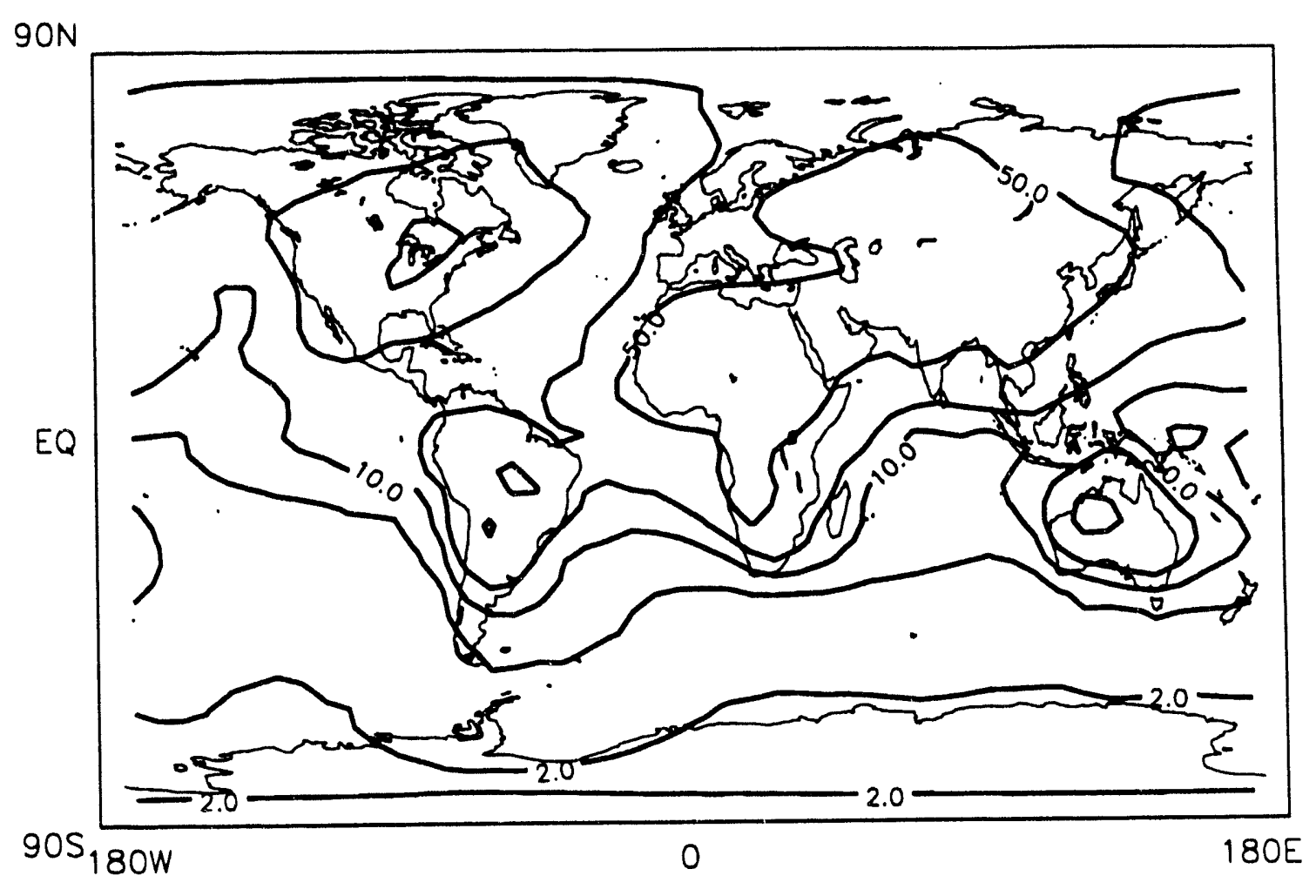


EULERIAN GRANTOUR-LLNL

A: $600 \mathrm{mb}$

LEAD - 210 Mixing Rotio $[* e 21 \mathrm{v} / \mathrm{v}]$

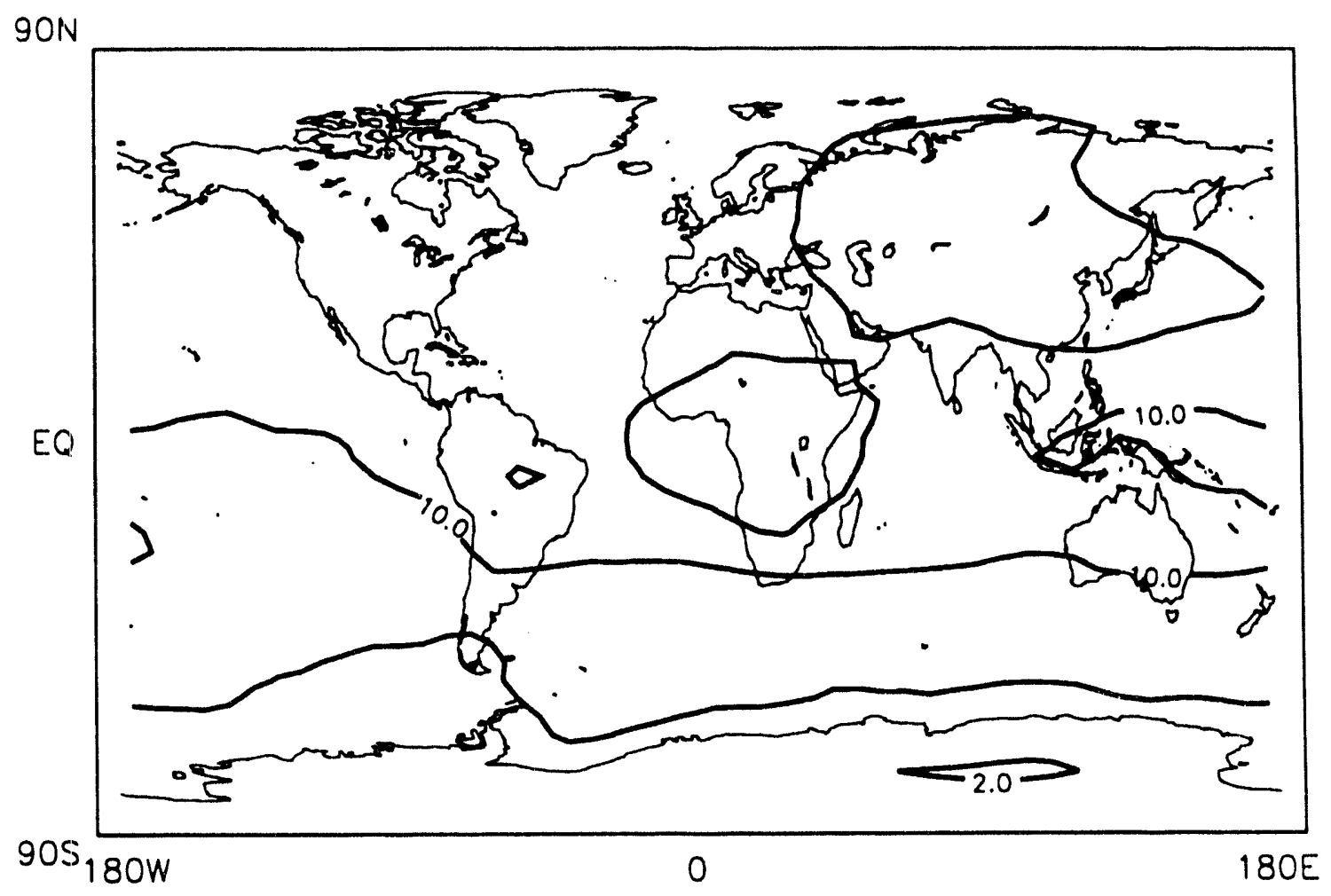


EULERIAN GRANTOUR-LLNL

DJF

A: $300 \mathrm{mb}$

LEAD-210 Mixing Ratio [*e21 v/v]

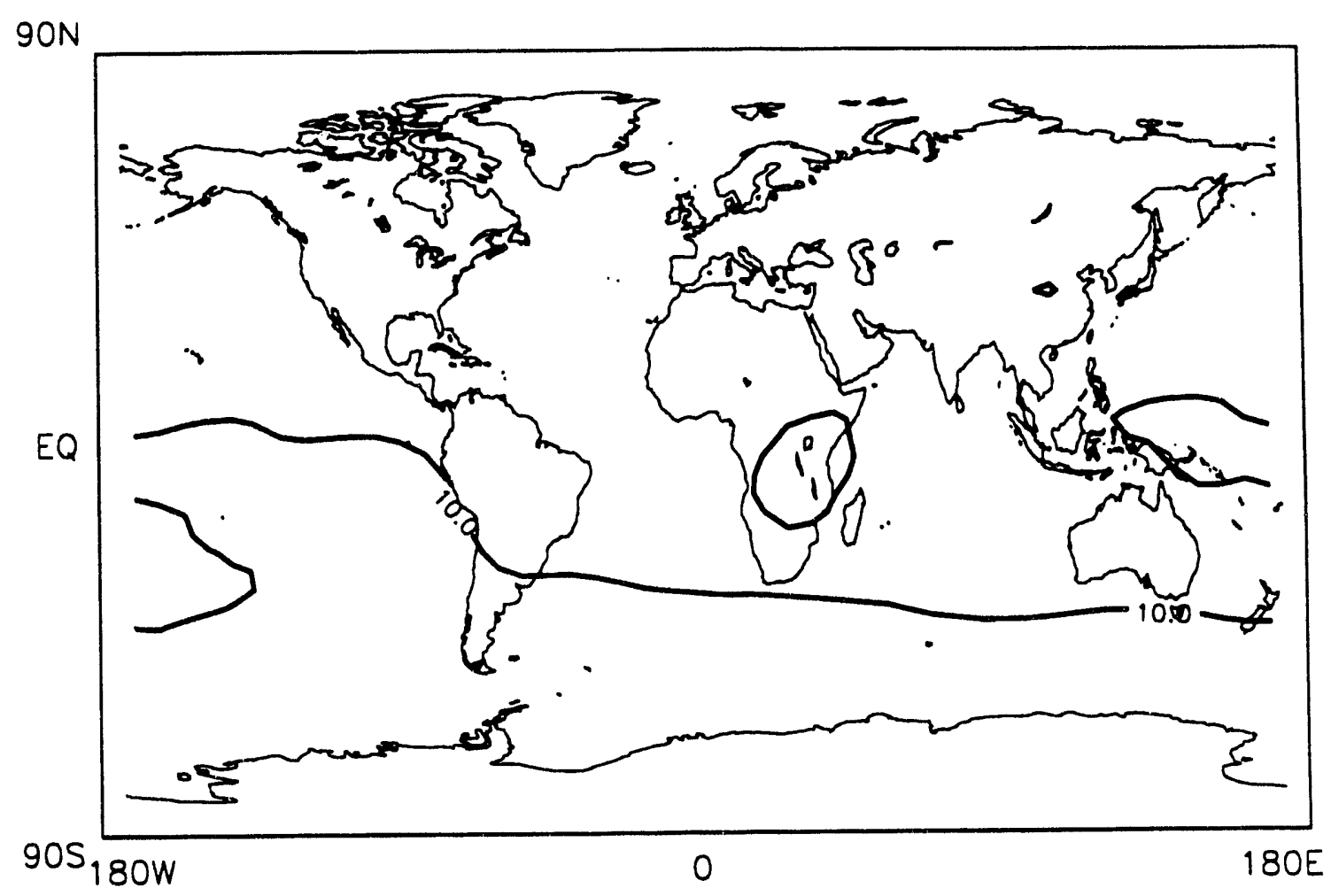



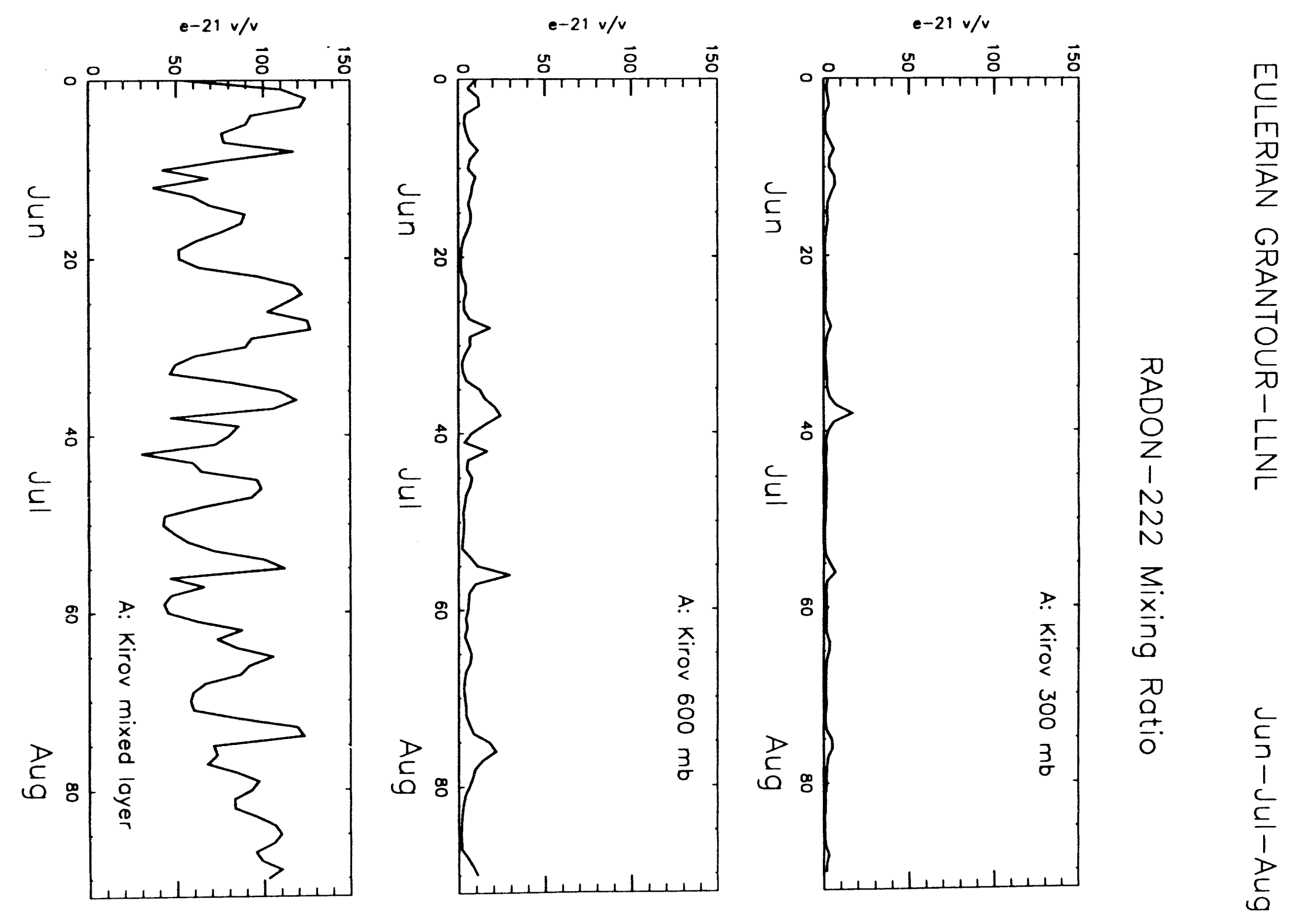
RADON-222 Mixing Ratio
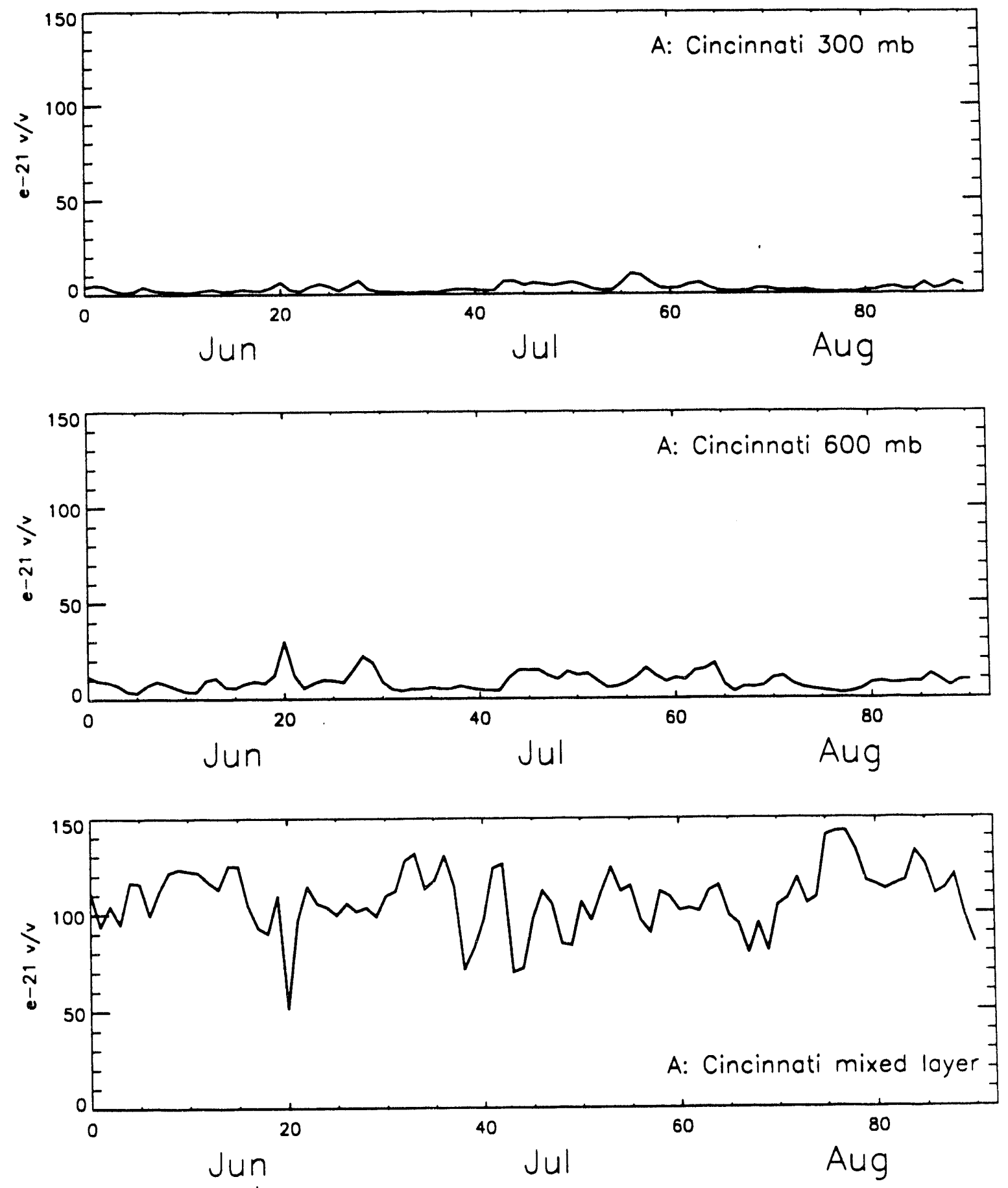
RADON-222 Mixing Ratio
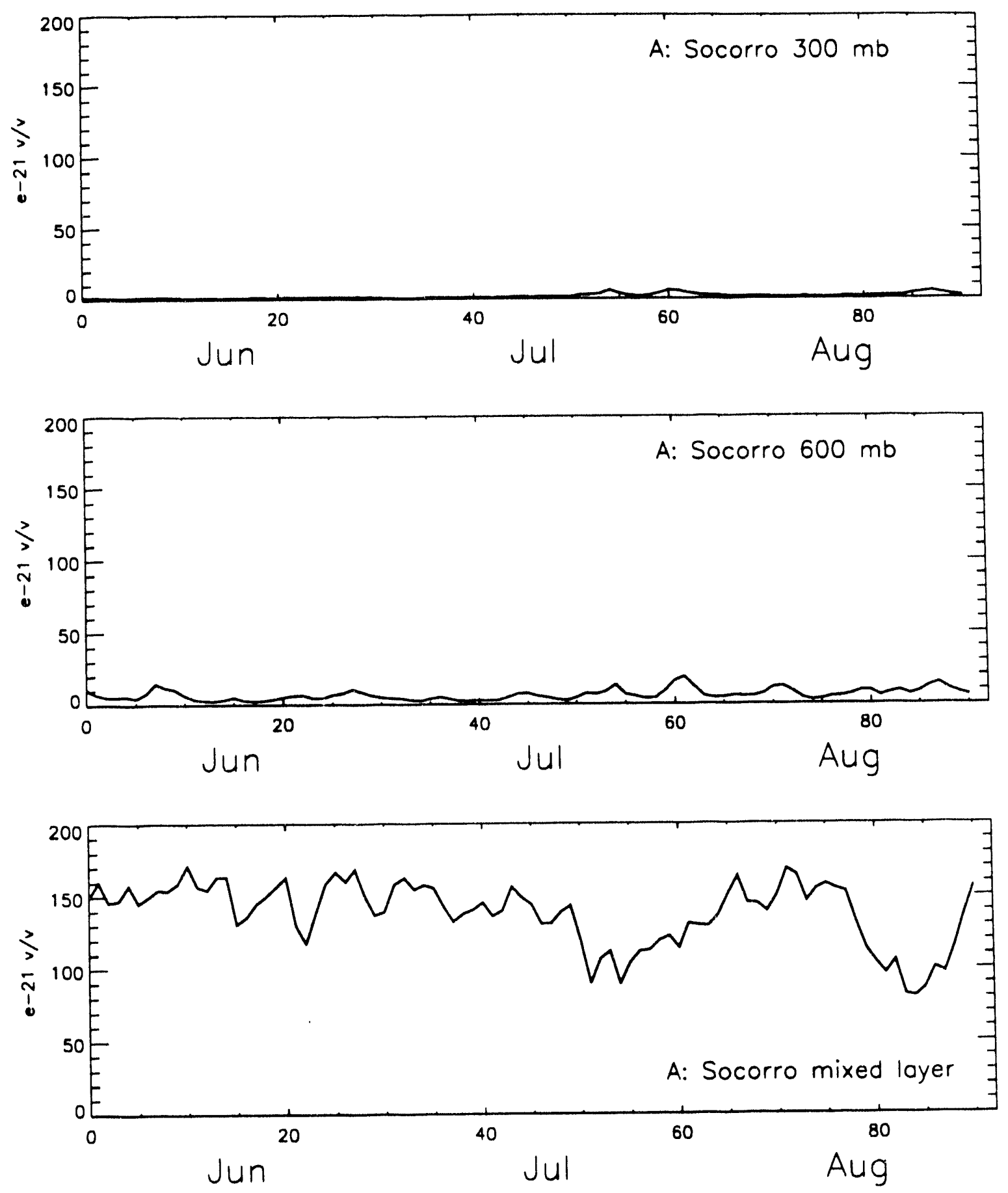
RADON-222 Mixing Ratio
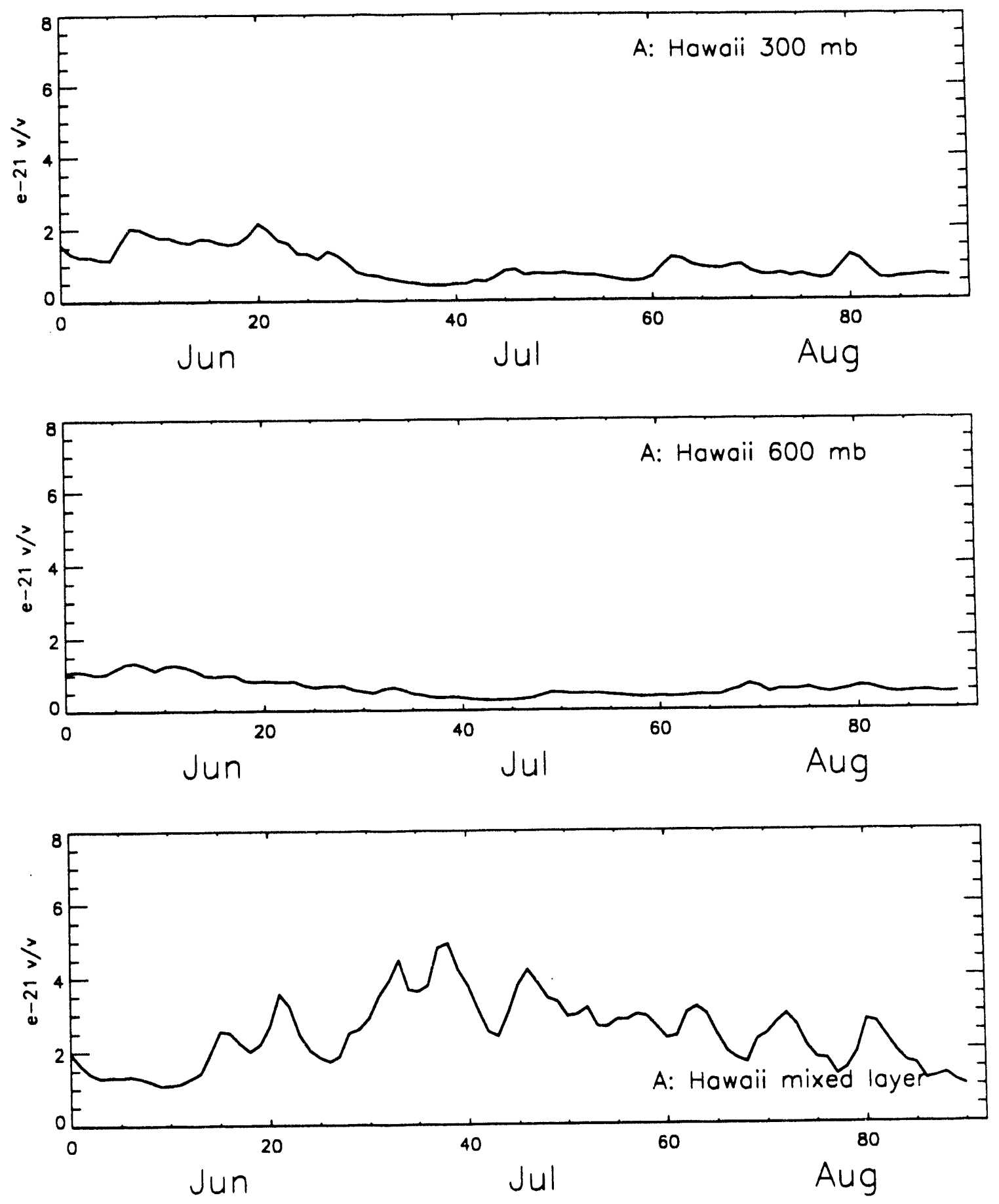
RADON-222 Mixing Ratio
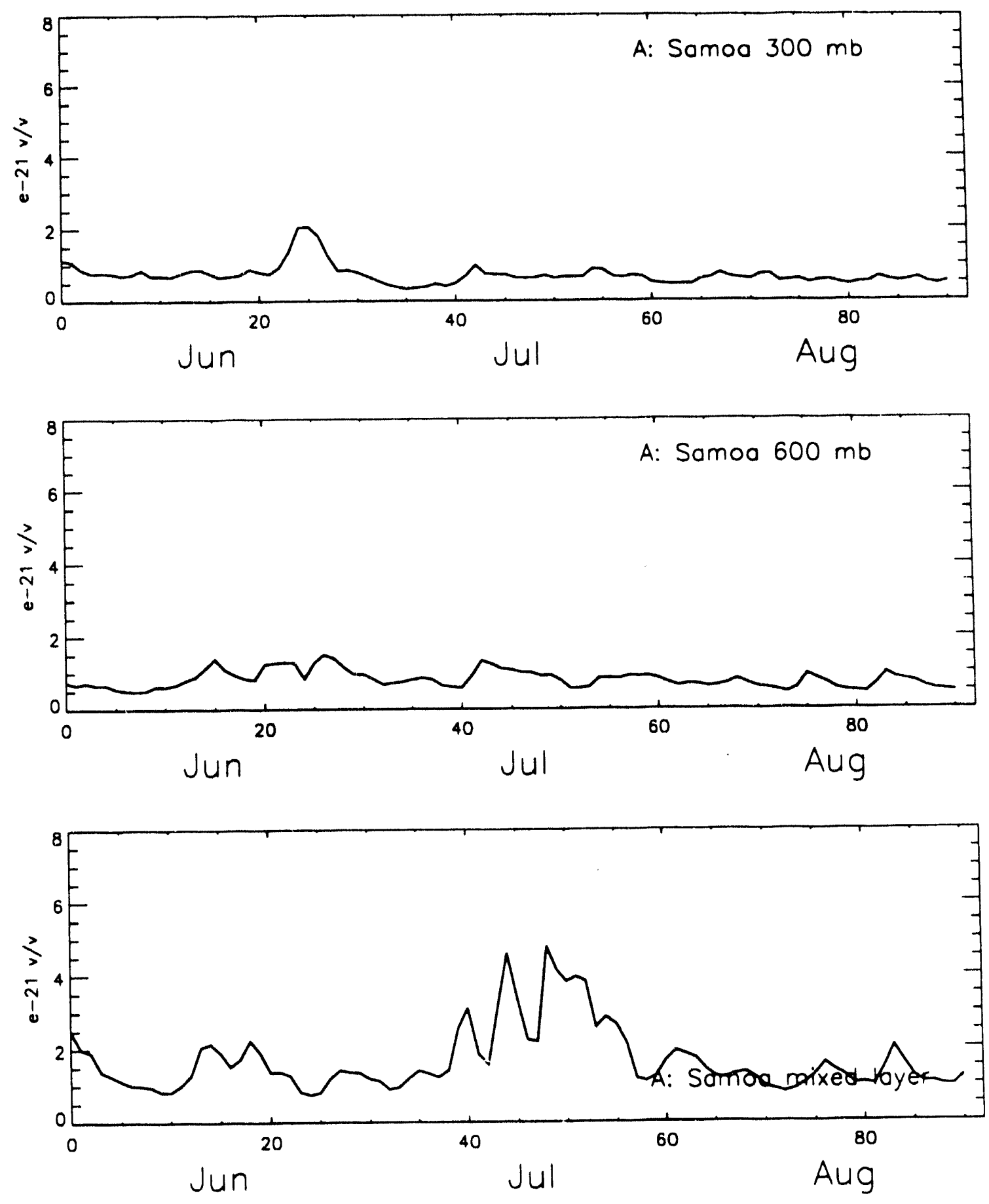
RADON-222 Mixing Ratio
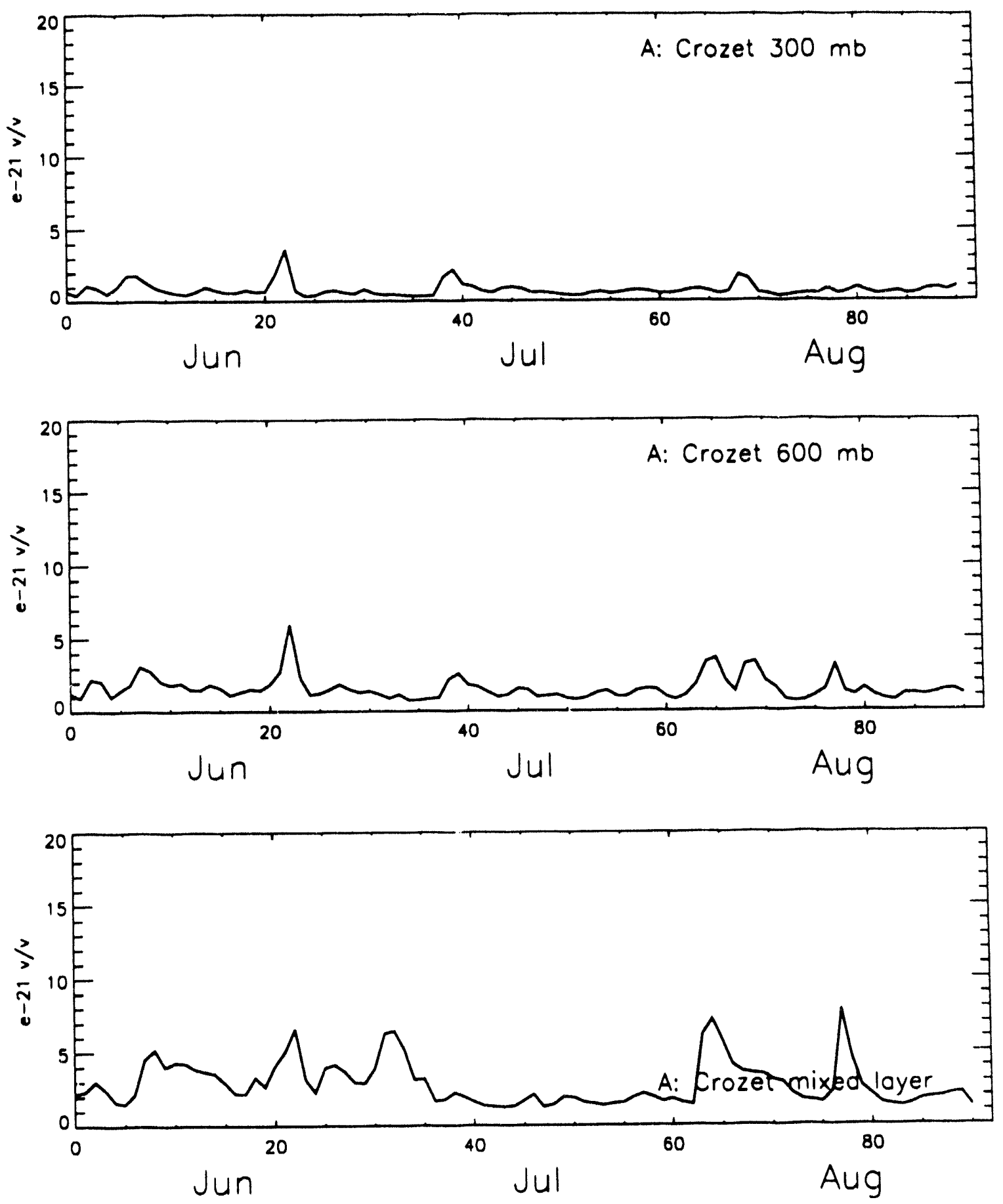


\section{RADON-222 Mixing Ratio}
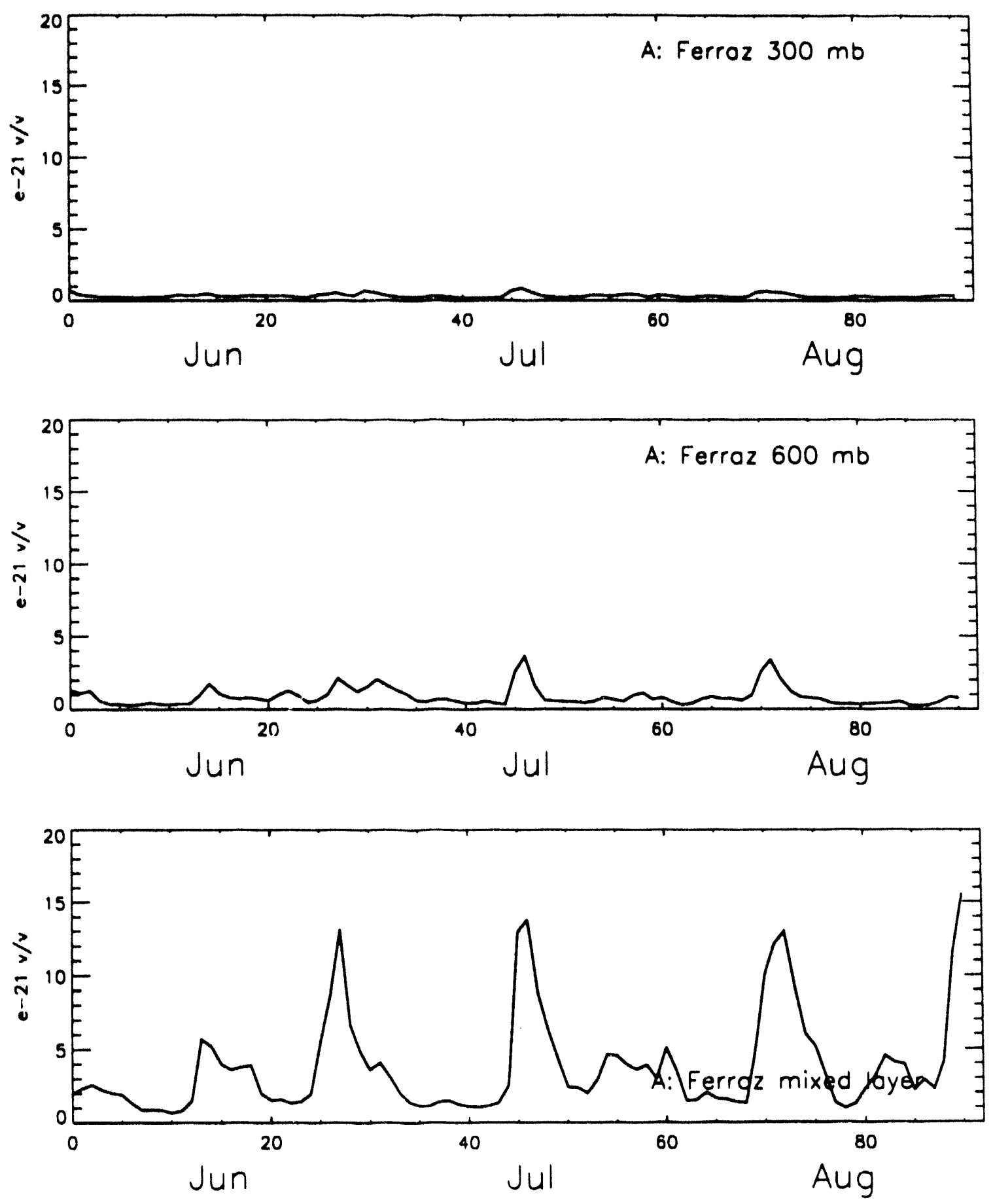


\begin{tabular}{|c|c|c|c|c|c|c|c|c|c|}
\hline \multicolumn{10}{|c|}{ EULERIAN GRANTOUR-LLNL Case A: Time series statistics (E-21 v/v) Jun-Jul-Aug } \\
\hline site & level & mean & stdv & $\operatorname{var}^{*} E-42$ & $\min$ & $1 / 4$ & median & $3 / 4$ & $\max$ \\
\hline Kirov & $\begin{array}{c}300 \mathrm{mb} \\
600 \mathrm{mb} \\
\text { mixed } \\
\text { surface }\end{array}$ & \begin{tabular}{r|}
2.34 \\
6.95 \\
81.46 \\
88.40 \\
\end{tabular} & \begin{tabular}{r|}
2.16 \\
5.36 \\
24.89 \\
24.18 \\
\end{tabular} & $\begin{array}{r}4.67 \\
28.74 \\
619.35 \\
584.67 \\
\end{array}$ & $\begin{array}{r}0.66 \\
1.41 \\
30.20 \\
46.05 \\
\end{array}$ & $\begin{array}{r}1.21 \\
3.47 \\
60.70 \\
68.77 \\
\end{array}$ & $\begin{array}{r}1.70 \\
5.49 \\
83.36 \\
88.06 \\
\end{array}$ & \begin{tabular}{r|}
2.56 \\
8.16 \\
100.05 \\
107.36 \\
\end{tabular} & \begin{tabular}{r|}
16.98 \\
29.70 \\
126.94 \\
131.42 \\
\end{tabular} \\
\hline Cincinnati & $\begin{array}{c}300 \mathrm{mb} \\
600 \mathrm{mb} \\
\text { mixed } \\
\text { surface }\end{array}$ & $\begin{array}{r}3.10 \\
8.73 \\
107.63 \\
118.22 \\
\end{array}$ & $\begin{array}{r}2.05 \\
4.50 \\
16.30 \\
16.60 \\
\end{array}$ & $\begin{array}{r}4.20 \\
20.24 \\
265.58 \\
275.40 \\
\end{array}$ & $\begin{array}{r}0.64 \\
2.25 \\
51.35 \\
73.86 \\
\end{array}$ & \begin{tabular}{r|}
1.62 \\
5.48 \\
98.84 \\
108.22 \\
\end{tabular} & $\begin{array}{r}2.38 \\
8.20 \\
109.90 \\
118.66 \\
\end{array}$ & $\begin{array}{r}4.37 \\
10.84 \\
116.87 \\
129.22 \\
\end{array}$ & $\begin{array}{r}10.82 \\
30.02 \\
142.76 \\
155.09 \\
\end{array}$ \\
\hline Socorro & $\begin{array}{c}300 \mathrm{mb} \\
600 \mathrm{mb} \\
\text { mixed } \\
\text { surface }\end{array}$ & $\begin{array}{r}1.77 \\
6.42 \\
138.12 \\
147.47 \\
\end{array}$ & $\begin{array}{r}1.14 \\
3.38 \\
22.32 \\
21.96 \\
\end{array}$ & $\begin{array}{r}1.31 \\
11.43 \\
498.31 \\
482.18 \\
\end{array}$ & \begin{tabular}{r|}
0.64 \\
2.37 \\
80.58 \\
92.72 \\
\end{tabular} & $\begin{array}{r}1.05 \\
4.10 \\
129.14 \\
134.32 \\
\end{array}$ & $\begin{array}{r}1.40 \\
5.42 \\
144.28 \\
153.12 \\
\end{array}$ & $\begin{array}{r}1.84 \\
7.87 \\
155.57 \\
164.91 \\
\end{array}$ & \begin{tabular}{r|}
6.01 \\
18.83 \\
171.08 \\
180.42 \\
\end{tabular} \\
\hline Hawaii & $\begin{array}{c}300 \mathrm{mb} \\
600 \mathrm{mb} \\
\text { mixed } \\
\text { surface }\end{array}$ & $\begin{array}{l}0.99 \\
0.62 \\
2.44 \\
2.61 \\
\end{array}$ & $\begin{array}{l}0.46 \\
0.29 \\
0.93 \\
1.01 \\
\end{array}$ & $\begin{array}{l}0.21 \\
0.08 \\
0.87 \\
1.02 \\
\end{array}$ & $\begin{array}{l}0.43 \\
0.27 \\
1.01 \\
1.09 \\
\end{array}$ & $\begin{array}{l}0.64 \\
0.41 \\
1.66 \\
1.79 \\
\end{array}$ & $\begin{array}{l}0.80 \\
0.51 \\
2.43 \\
2.62 \\
\end{array}$ & $\begin{array}{l}1.30 \\
0.81 \\
2.97 \\
3.17 \\
\end{array}$ & $\begin{array}{l}2.15 \\
1.34 \\
4.93 \\
5.34 \\
\end{array}$ \\
\hline Samoa & $\begin{array}{c}300 \mathrm{mb} \\
600 \mathrm{mb} \\
\text { mixed } \\
\text { surface }\end{array}$ & $\begin{array}{l}0.71 \\
0.80 \\
1.66 \\
1.79 \\
\end{array}$ & $\begin{array}{l}0.30 \\
0.25 \\
0.88 \\
0.95 \\
\end{array}$ & \begin{tabular}{l|}
0.09 \\
0.06 \\
0.78 \\
0.91 \\
\end{tabular} & \begin{tabular}{l|}
0.33 \\
0.44 \\
0.75 \\
0.82 \\
\end{tabular} & \begin{tabular}{l|}
0.53 \\
0.61 \\
1.06 \\
1.18 \\
\end{tabular} & $\begin{array}{l}0.66 \\
0.77 \\
1.35 \\
1.44 \\
\end{array}$ & $\begin{array}{l}0.77 \\
0.92 \\
1.94 \\
2.07 \\
\end{array}$ & $\begin{array}{l}2.05 \\
1.50 \\
4.78 \\
4.97 \\
\end{array}$ \\
\hline Crozet & $\begin{array}{c}300 \mathrm{mb} \\
600 \mathrm{mb} \\
\text { mixed } \\
\text { surface }\end{array}$ & $\begin{array}{l}0.77 \\
1.54 \\
2.82 \\
2.87 \\
\end{array}$ & $\begin{array}{l}0.48 \\
0.81 \\
1.52 \\
1.54 \\
\end{array}$ & $\begin{array}{l}0.23 \\
0.66 \\
2.30 \\
2.38 \\
\end{array}$ & $\begin{array}{l}0.30 \\
0.59 \\
1.26 \\
1.27 \\
\end{array}$ & $\begin{array}{l}0.50 \\
1.02 \\
1.64 \\
1.66 \\
\end{array}$ & \begin{tabular}{l|}
0.64 \\
1.37 \\
2.20 \\
2.27 \\
\end{tabular} & $\begin{array}{l}0.84 \\
1.82 \\
3.68 \\
3.73 \\
\end{array}$ & $\begin{array}{l}3.58 \\
5.96 \\
7.84 \\
7.75 \\
\end{array}$ \\
\hline Ferraz & $\begin{array}{c}300 \mathrm{mb} \\
600 \mathrm{mb} \\
\text { mixed } \\
\text { surface }\end{array}$ & $\begin{array}{l}0.34 \\
0.86 \\
3.80 \\
3.79 \\
\end{array}$ & \begin{tabular}{l|}
0.14 \\
0.64 \\
3.37 \\
3.33 \\
\end{tabular} & \begin{tabular}{r|}
0.02 \\
0.41 \\
11.35 \\
11.08 \\
\end{tabular} & \begin{tabular}{l|}
0.18 \\
0.23 \\
0.66 \\
0.77 \\
\end{tabular} & \begin{tabular}{l|}
0.25 \\
0.43 \\
1.48 \\
1.41 \\
\end{tabular} & $\begin{array}{l}0.30 \\
0.67 \\
2.51 \\
2.51\end{array}$ & $\begin{array}{l}0.37 \\
1.04 \\
4.54 \\
4.62 \\
\end{array}$ & $\begin{array}{r}0.87 \\
3.61 \\
15.53 \\
15.49 \\
\end{array}$ \\
\hline
\end{tabular}


EULERIAN GRANTOUR - LLNL JJA

\author{
A: Mixed Loyer
}

RADON-222 Mixing Rotio [*e21 v/v]

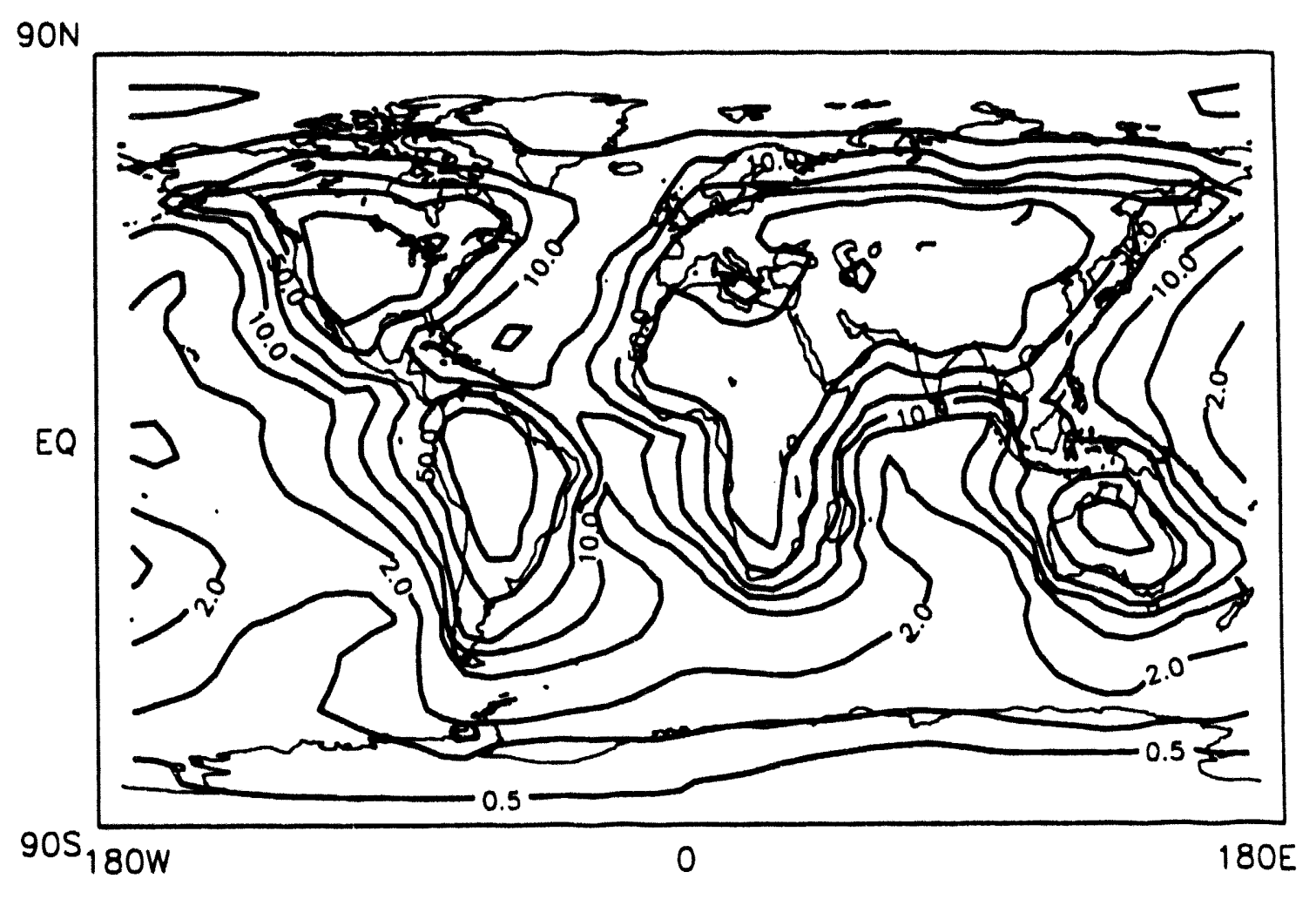


EULERIAN GRANTOUR-LLNL

JJA
A: $600 \mathrm{mb}$

RADON-222 Mixing Ratio [*e21 v/v]

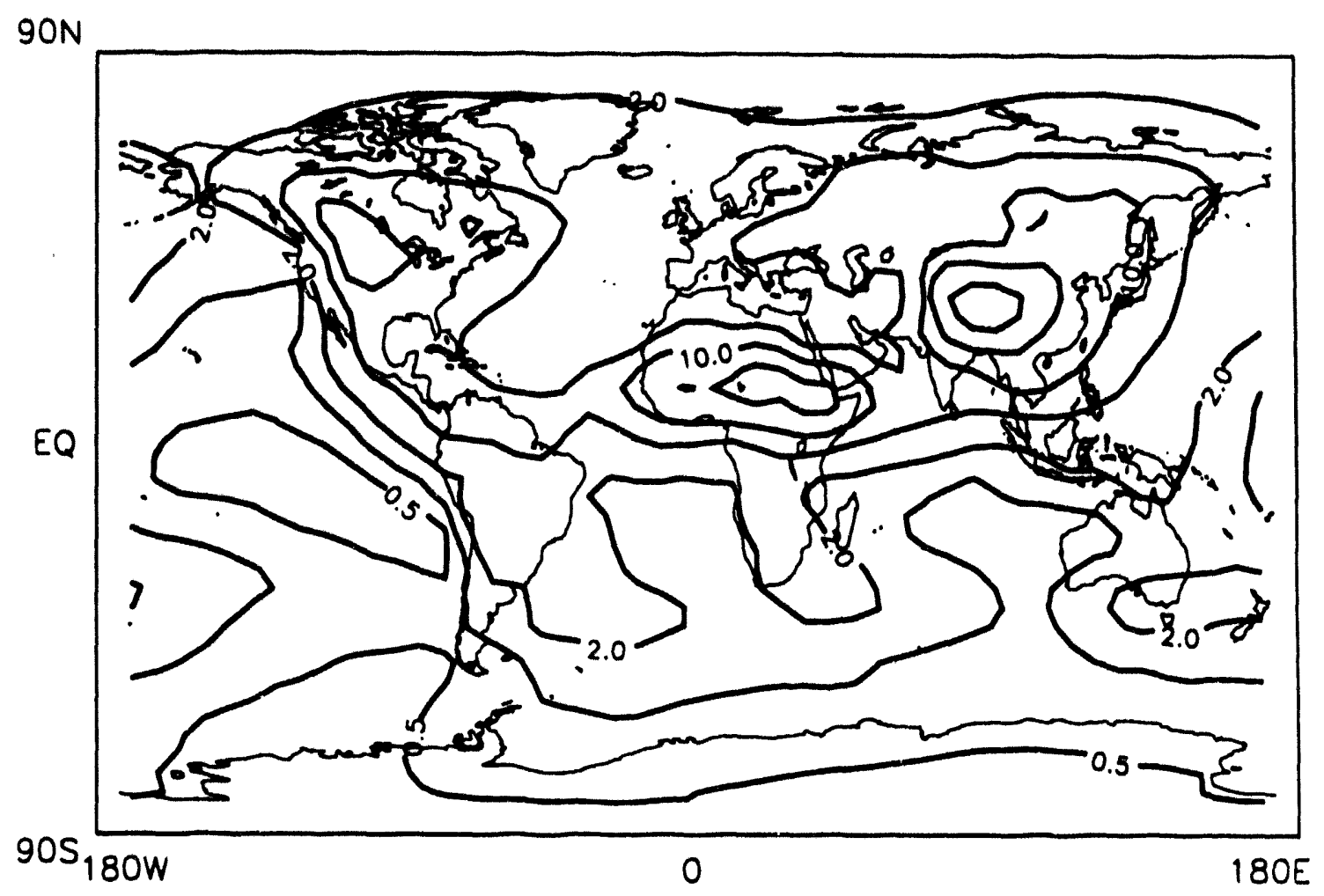


EULERIAN GRANTOUR-LLNL

JJA

\author{
A: $300 \mathrm{mb}$
}

RADON-222 Mixing Rotio [*e21 v/v]

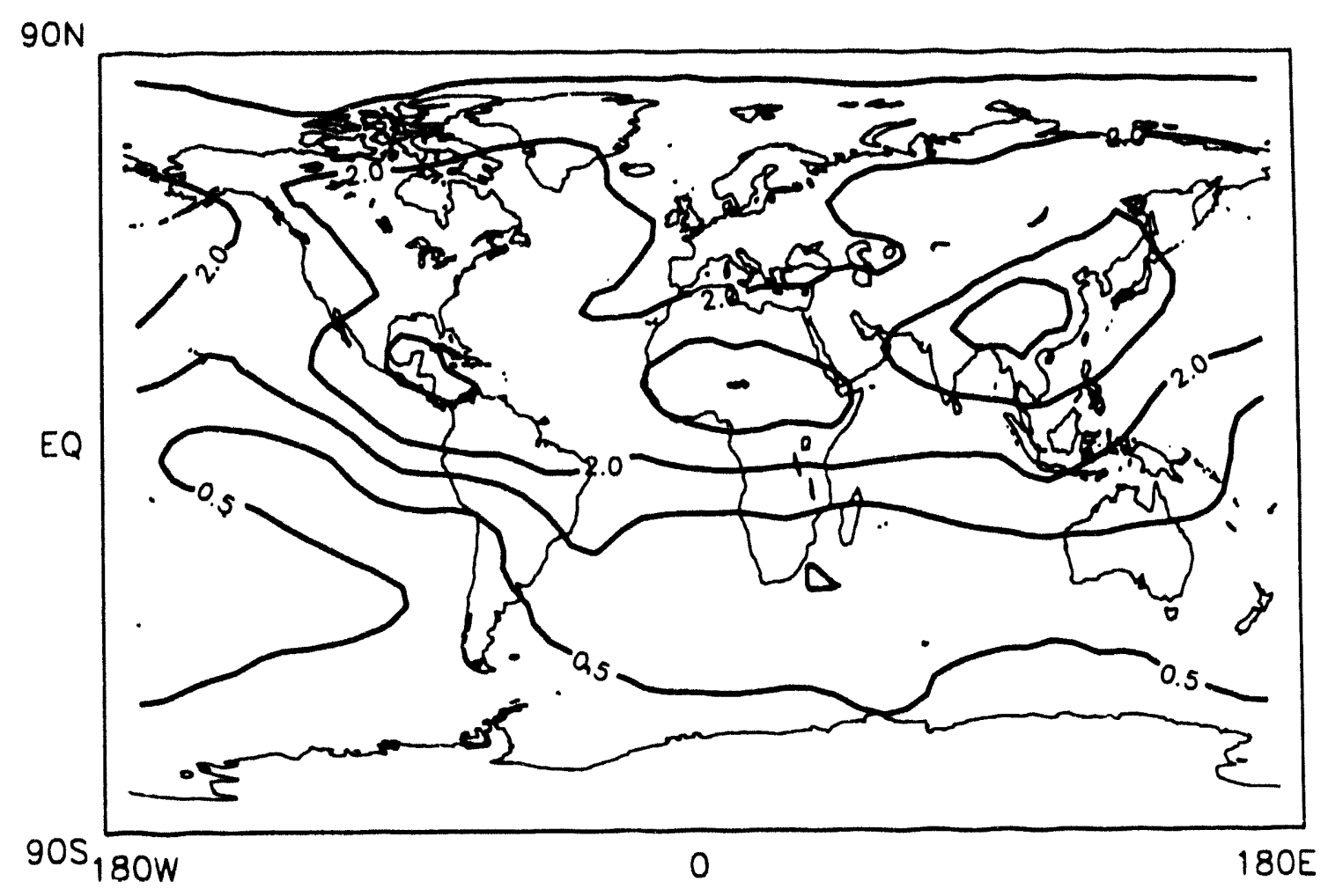


EULERIAN GRANTOUR-LLNL

JJA

\section{A: Zonal Avg}

RADON-222 Mixing Ratio [*e21 v/v]

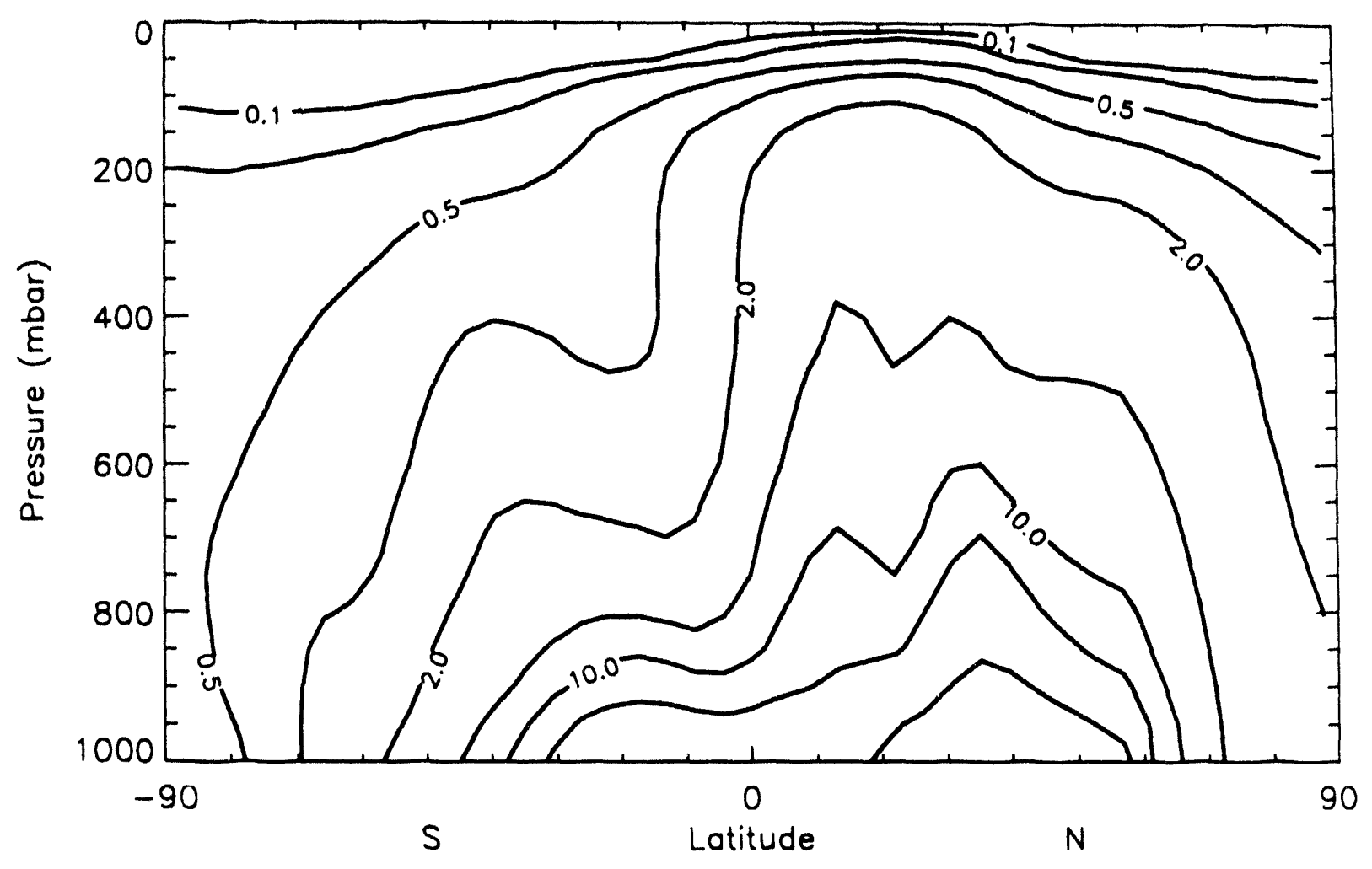


EULERIAN GRANTOUR-LLNL

JJA

\author{
A: OEW
}

RADON-222 Mixing Ratio $[* e 21 \mathrm{v} / \mathrm{v}]$

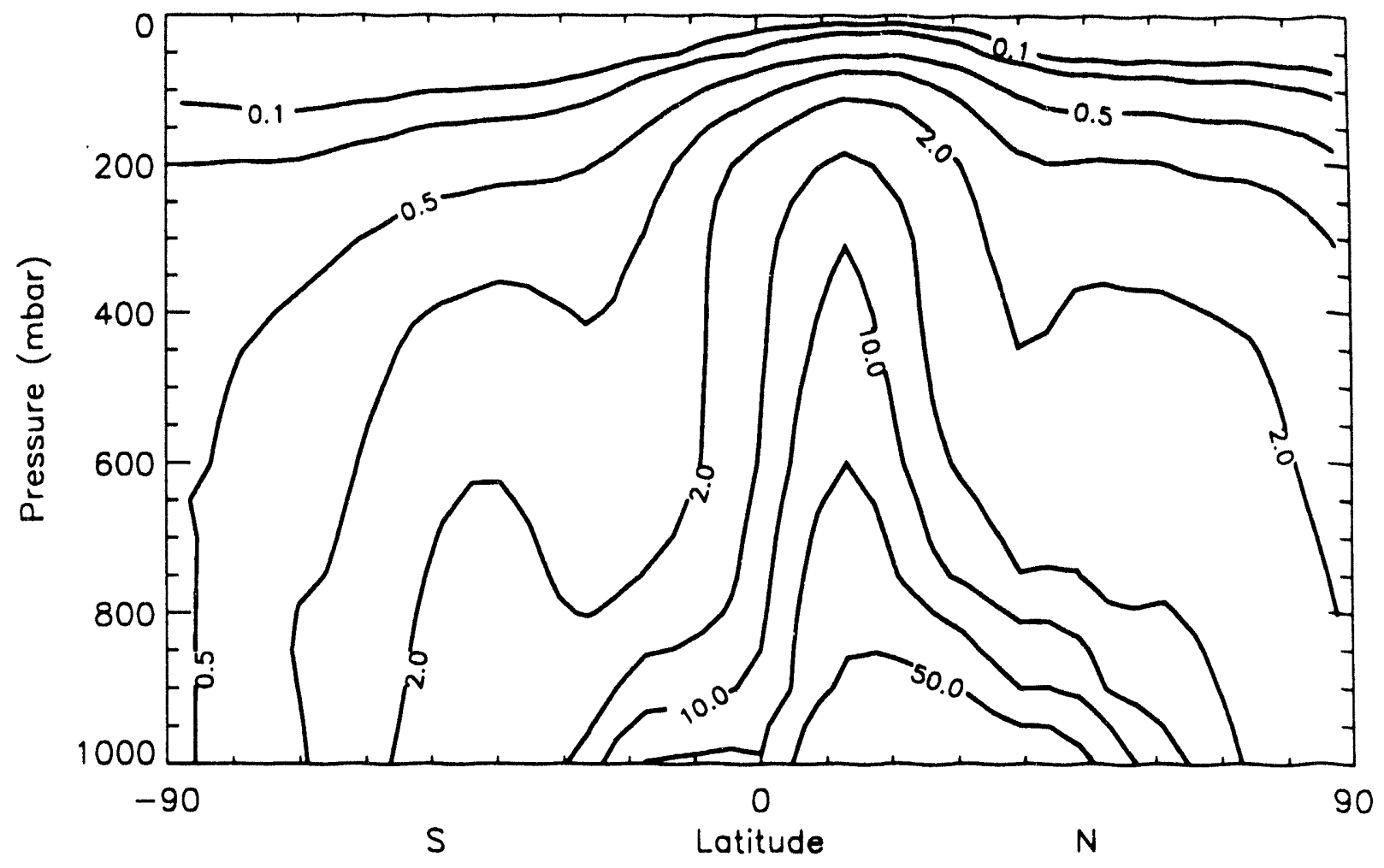


EULERIAN GRANTOUR-LLNL

JJA

\section{A: $180 E W$}

\section{RADON-222 Mixing Ratio [*e21 v/v]}

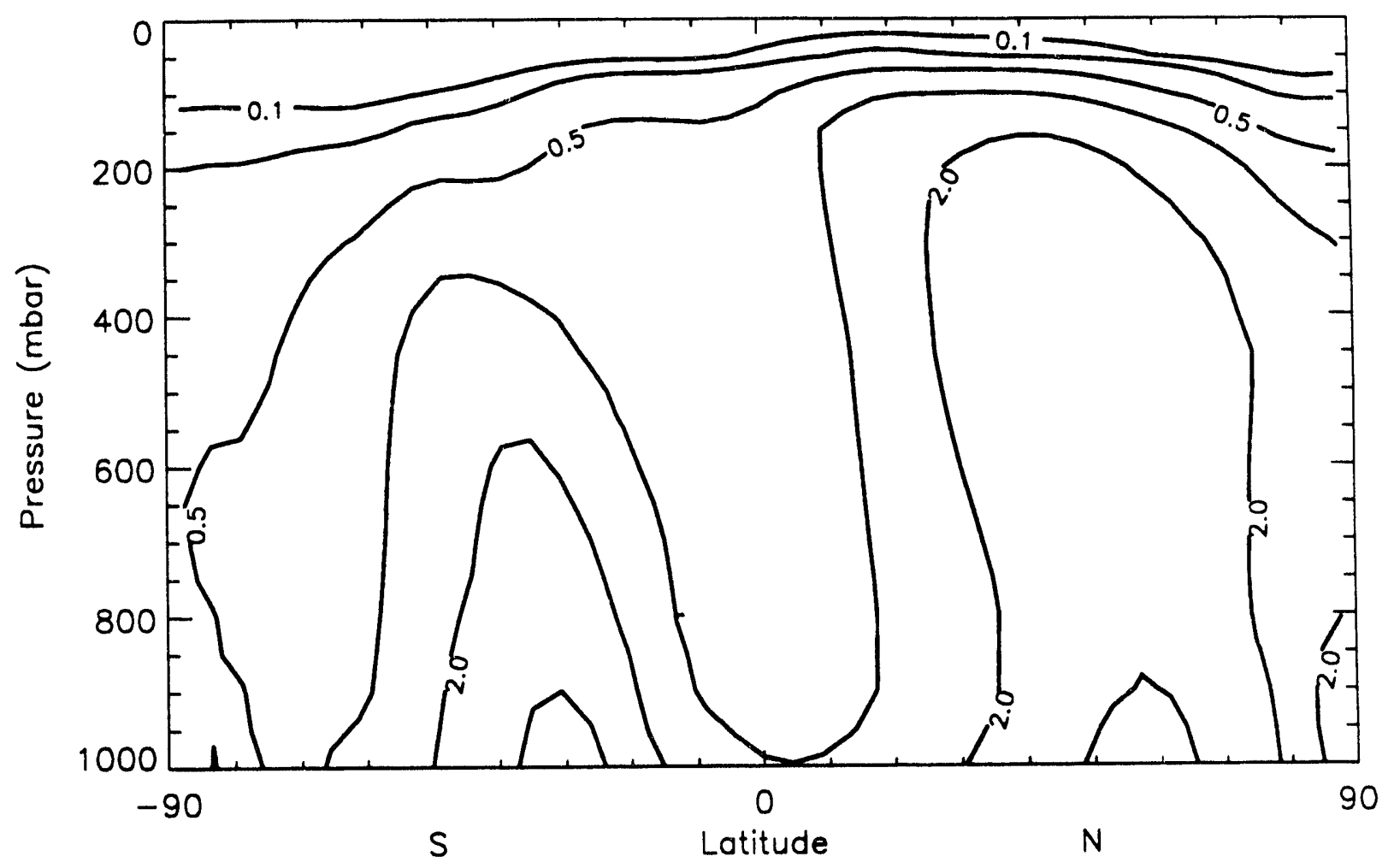


EULERIAN GRANTOUR-LLNL

JJA

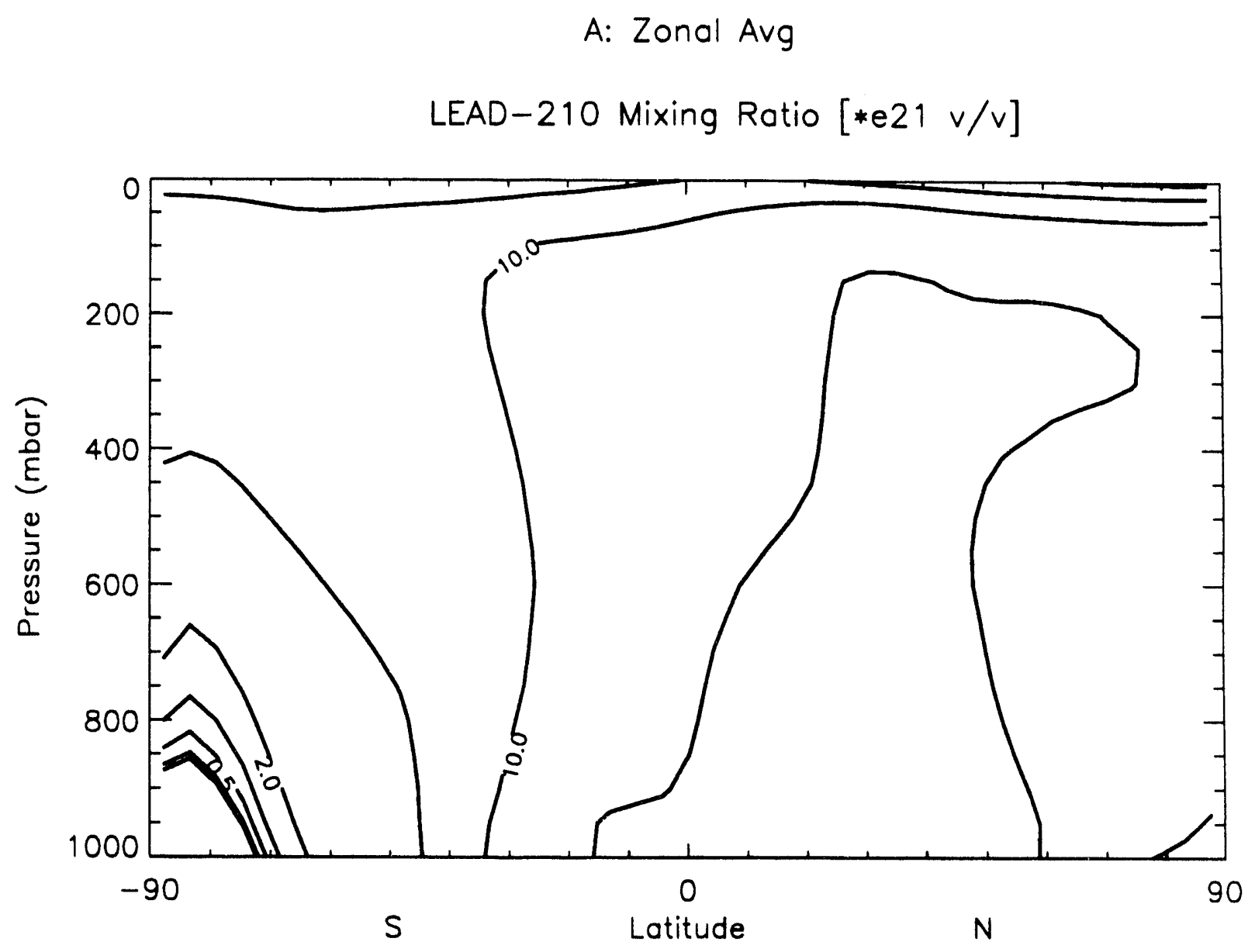


EULERIAN GRANTOUR-LLNL

JJA

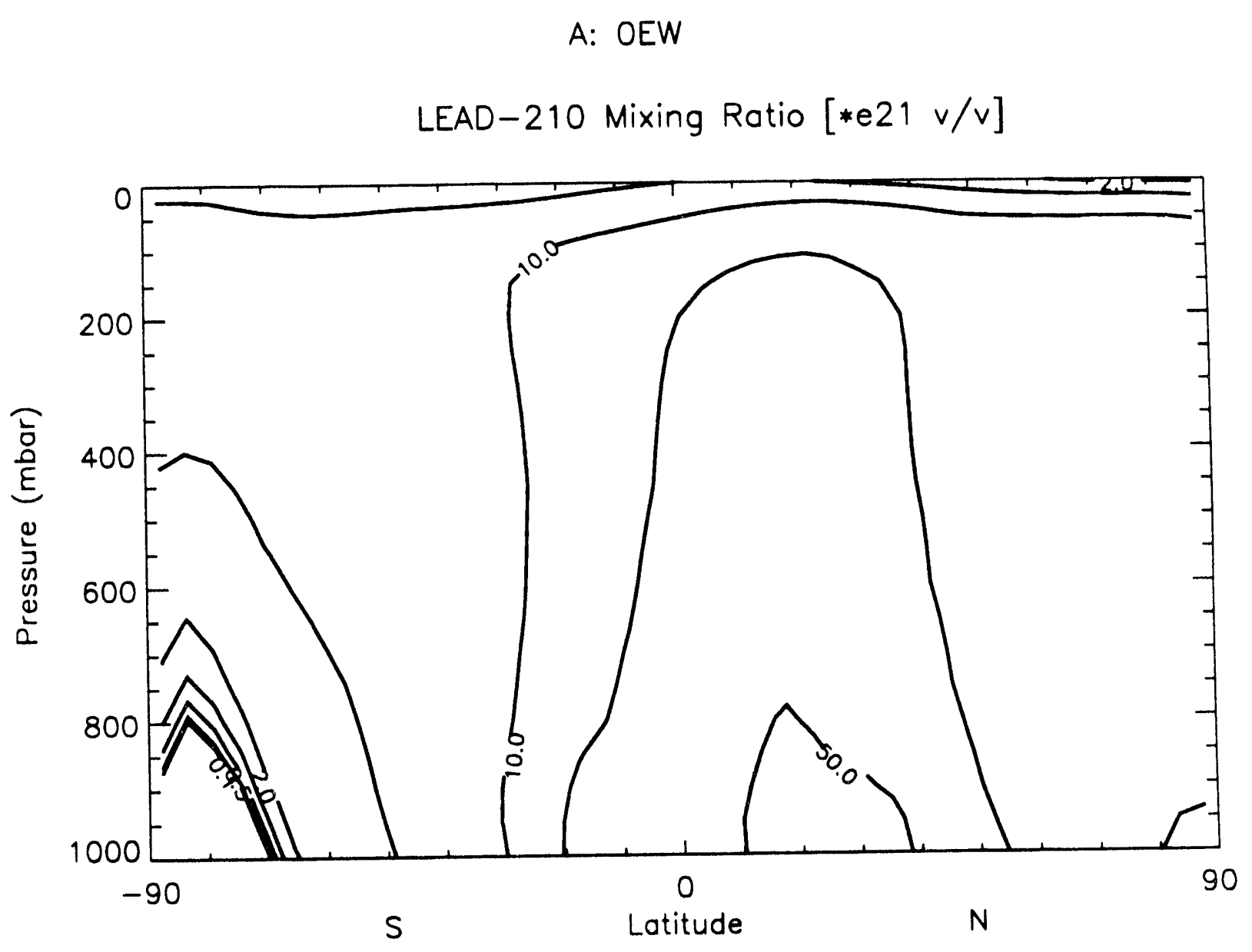


EULERIAN GRANTOUR-LLNL

JJA

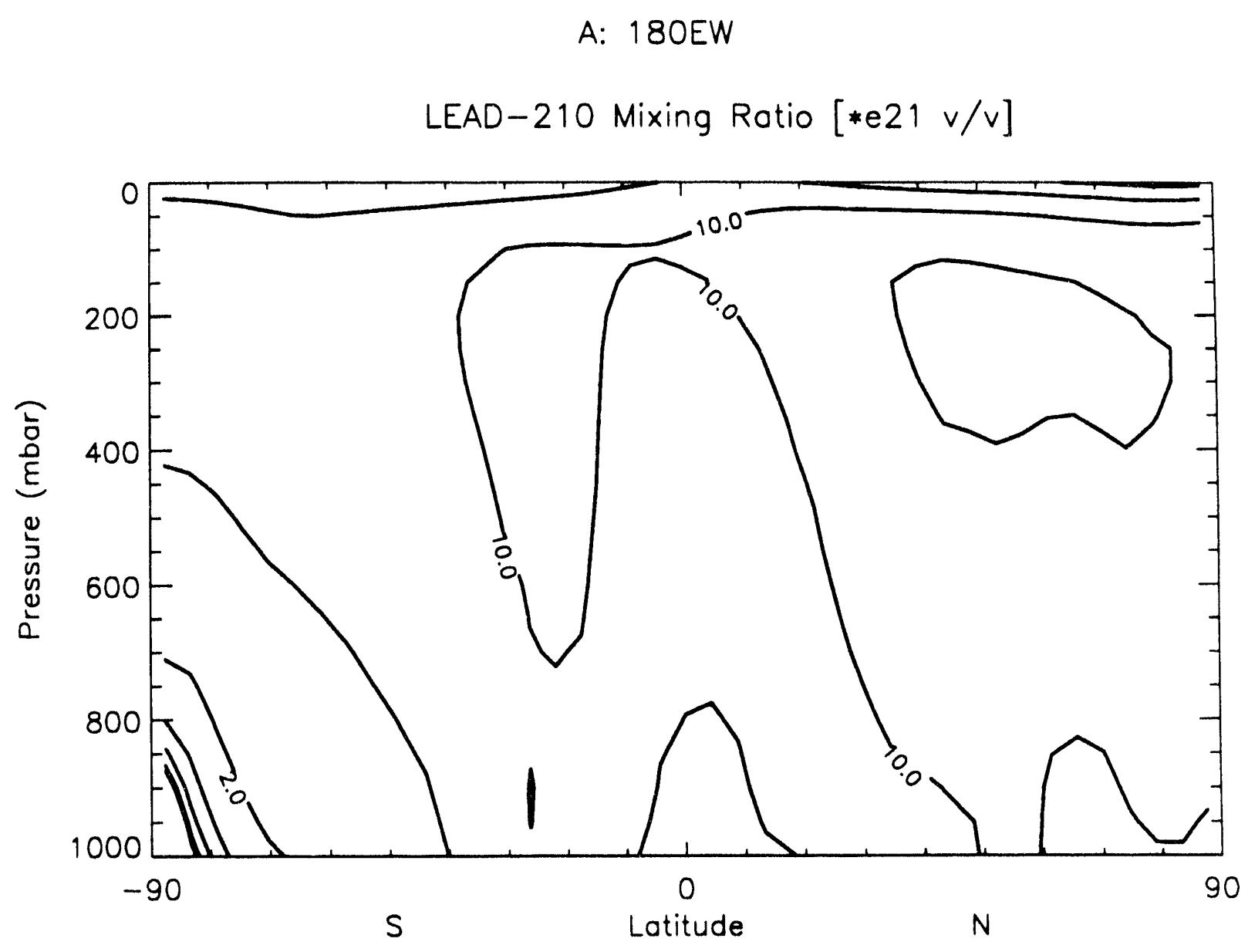


EULERIAN GRANTOUR-LLNL

JJA

\author{
A: Mixed Layer
}

LEAD-210 Mixing Ratio [*e21 v/v]

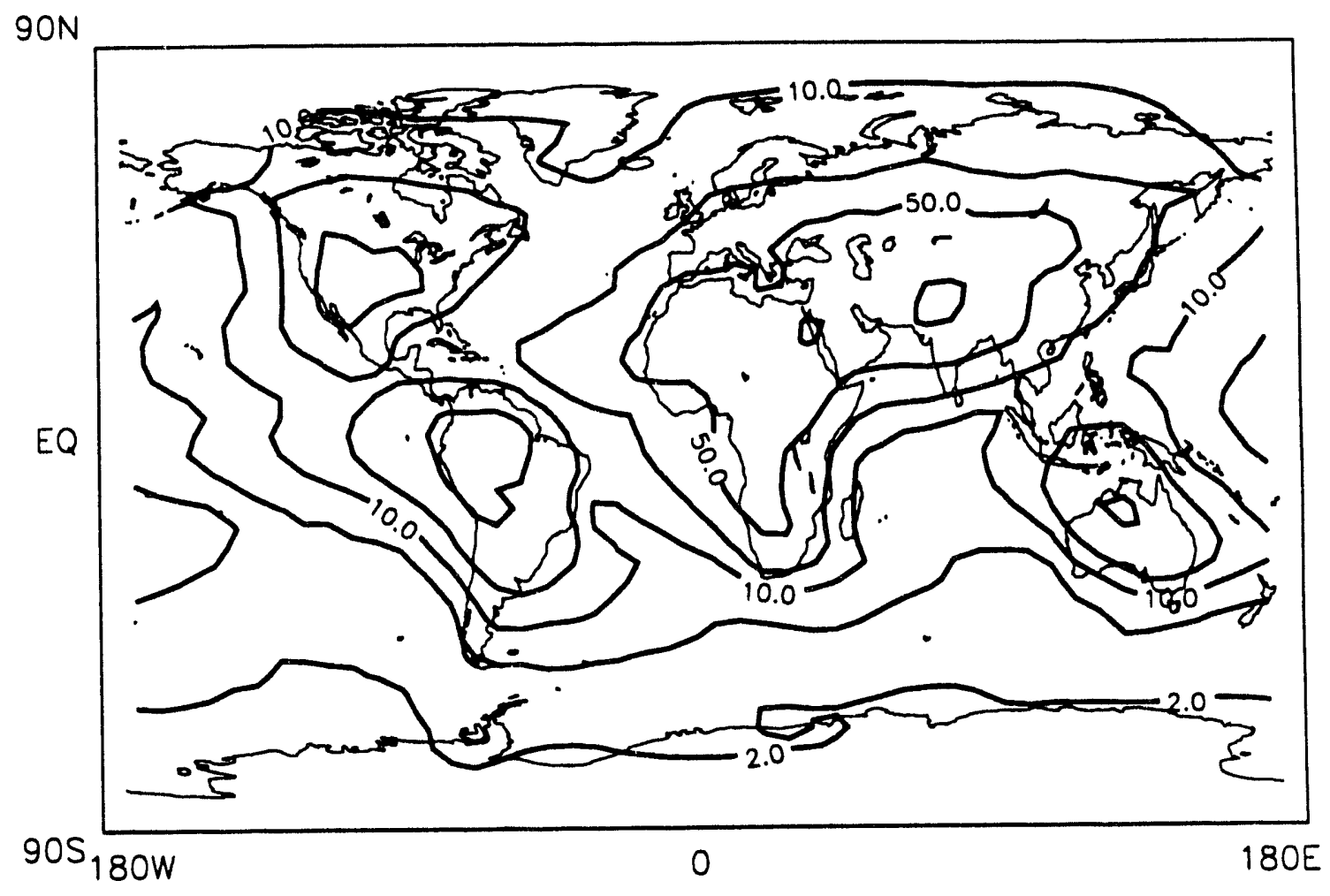


EULERIAN GRANTOUR-LLNL

JJA

A: $600 \mathrm{mb}$

LEAD-210 Mixing Ratio [*e21 v/v]

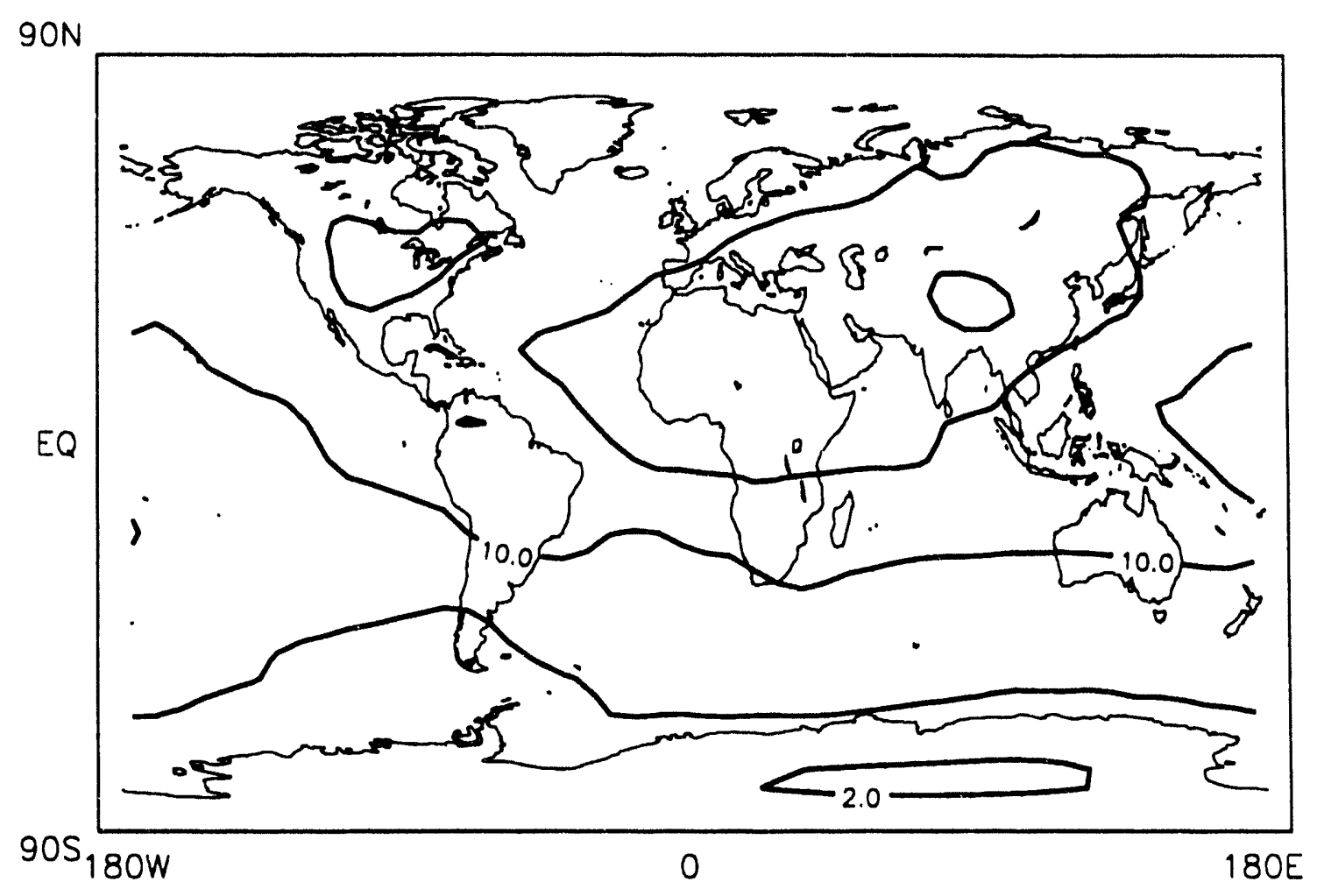


EULERIAN GRANTOUR-LLNL

JJA

A: $300 \mathrm{mb}$

LEAD-210 Mixing Ratio [*e21 v/v]

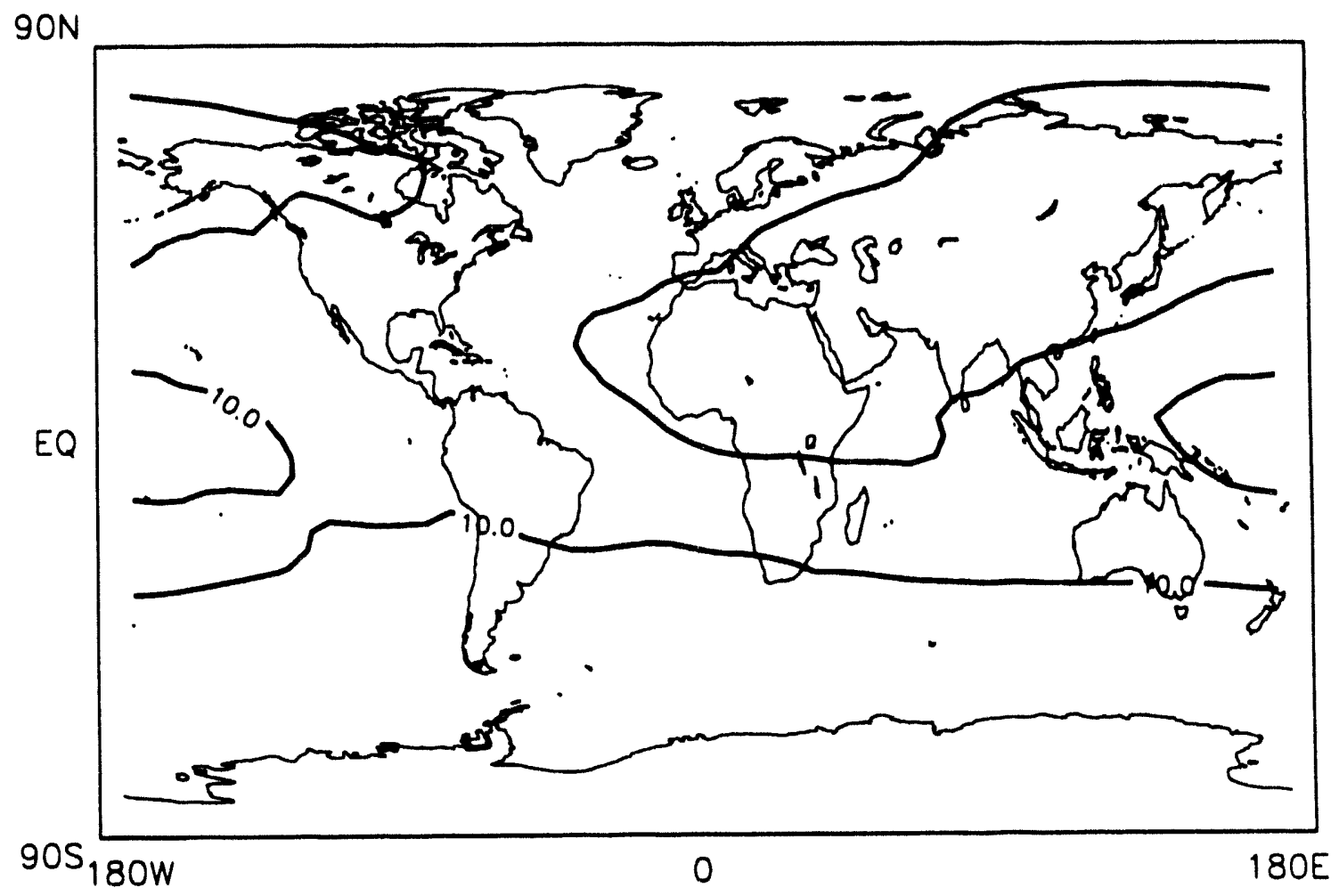




\begin{tabular}{|c|c|c|}
\hline EULERIAN GRANTOUR-LLNL & \multicolumn{2}{|c|}{ Scenario A: Lead Loading in kg } \\
\hline & Dec-Jan-Feb & Jun-Jul-Aug \\
\hline$<300 \mathrm{mb}$ & 0.199 & 0.220 \\
$<600 \mathrm{mb}$ & 0.379 & 0.417 \\
Total in Atmosphere & 0.617 & 0.670 \\
\hline
\end{tabular}


RADON-222 Mixing Ratio
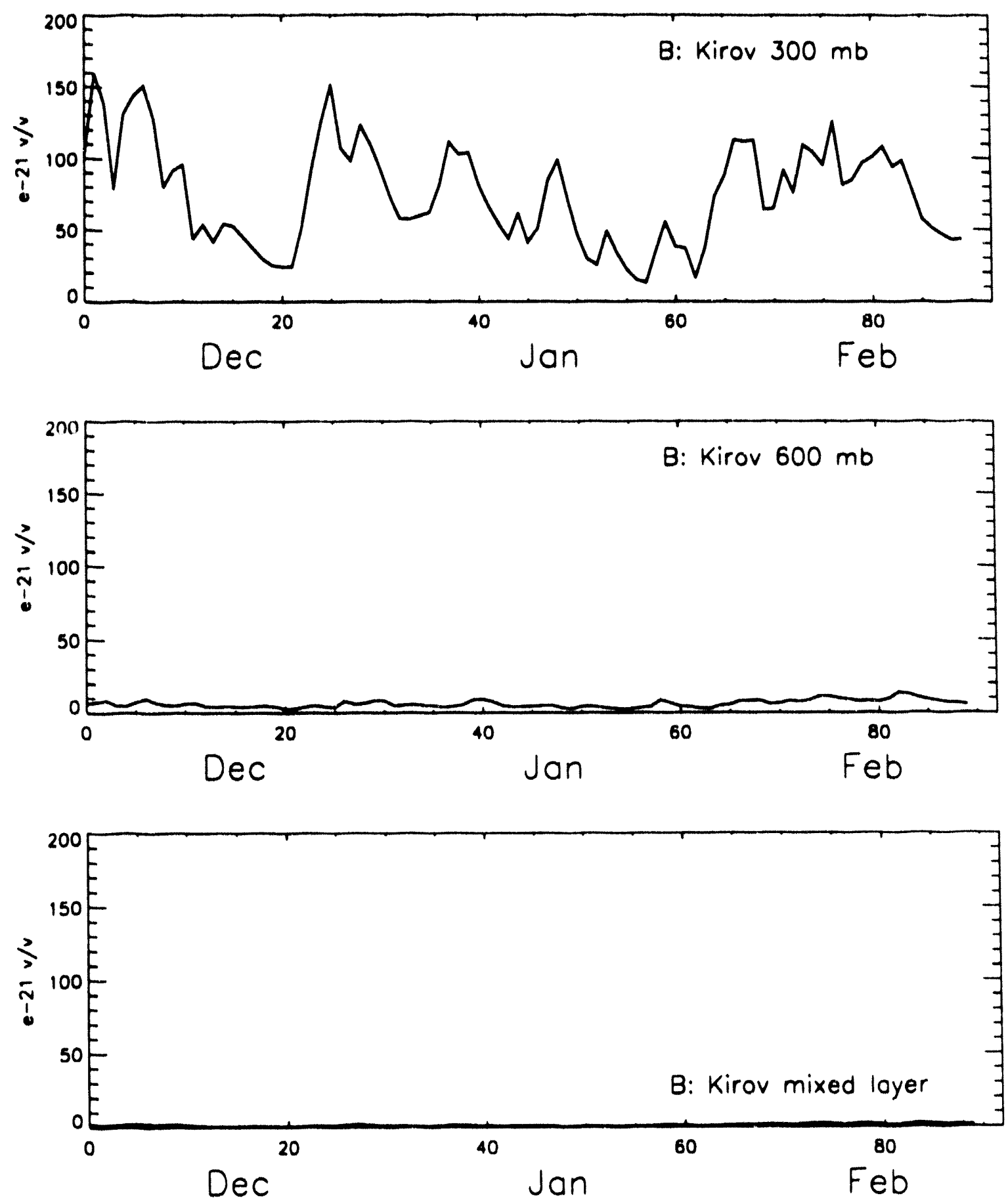


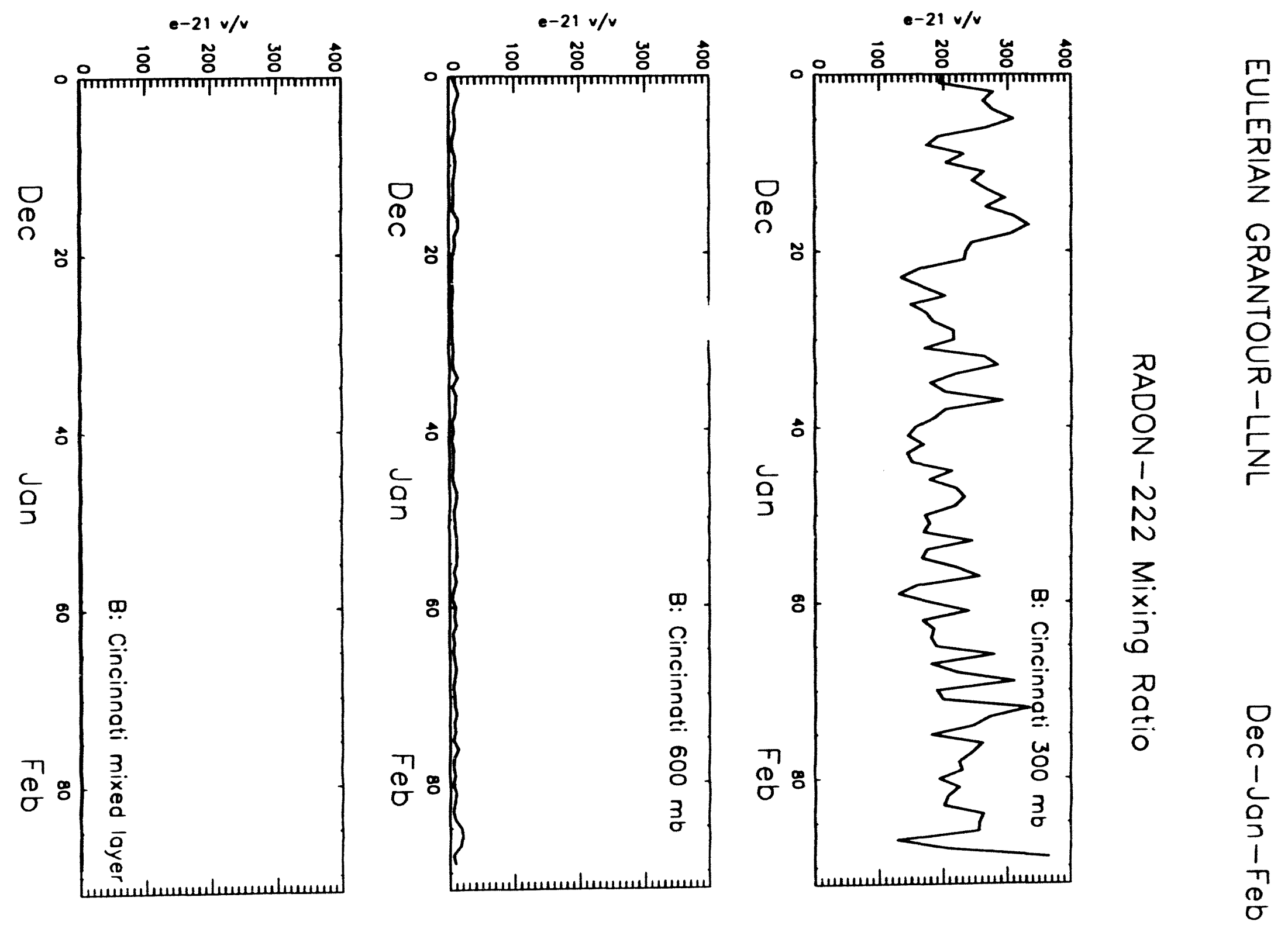


RADON-222 Mixing Ratio
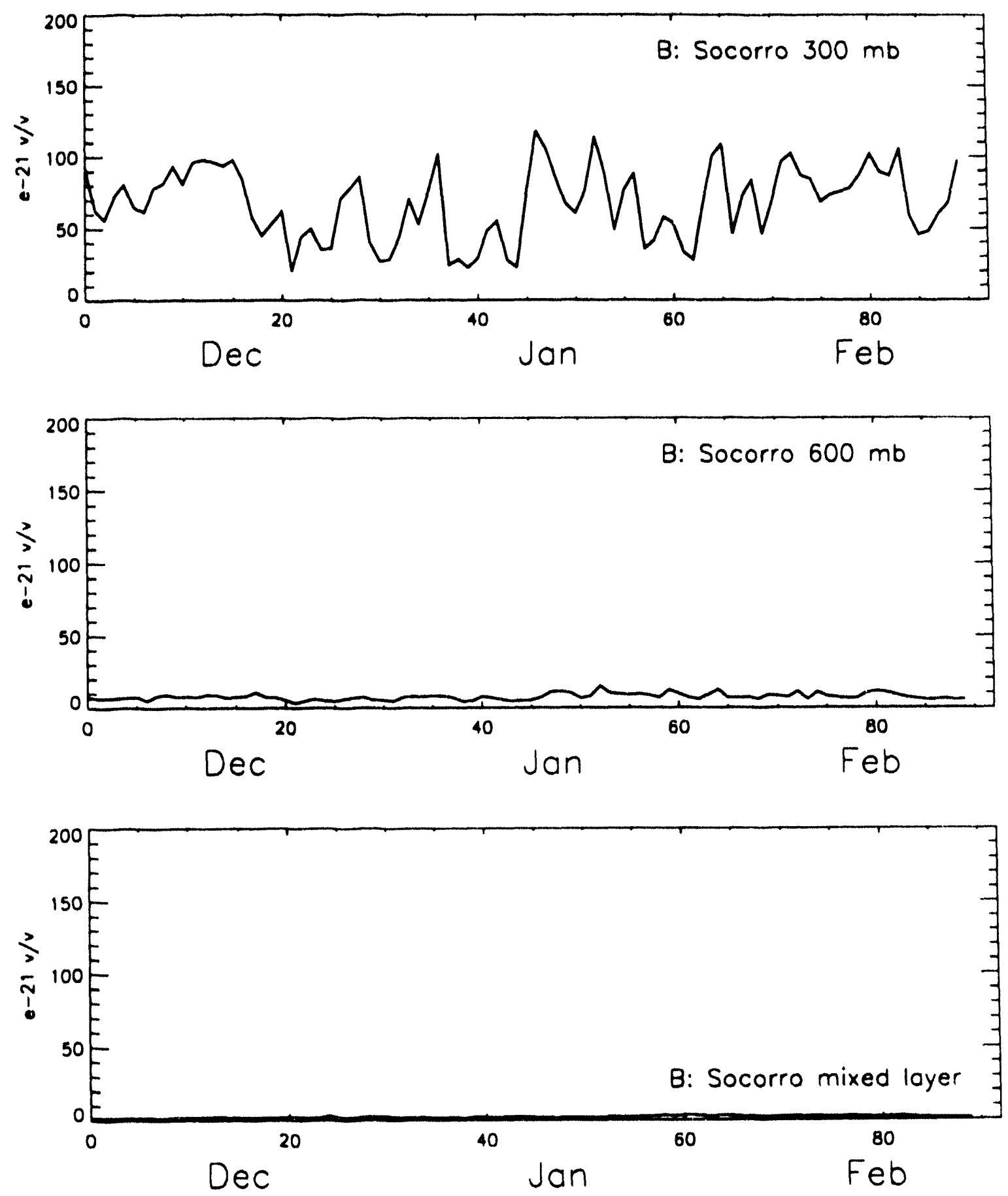
EULERIAN GRANTOUR-LLNL

Dec-Jan-Feb

RADON-222 Mixing Ratio
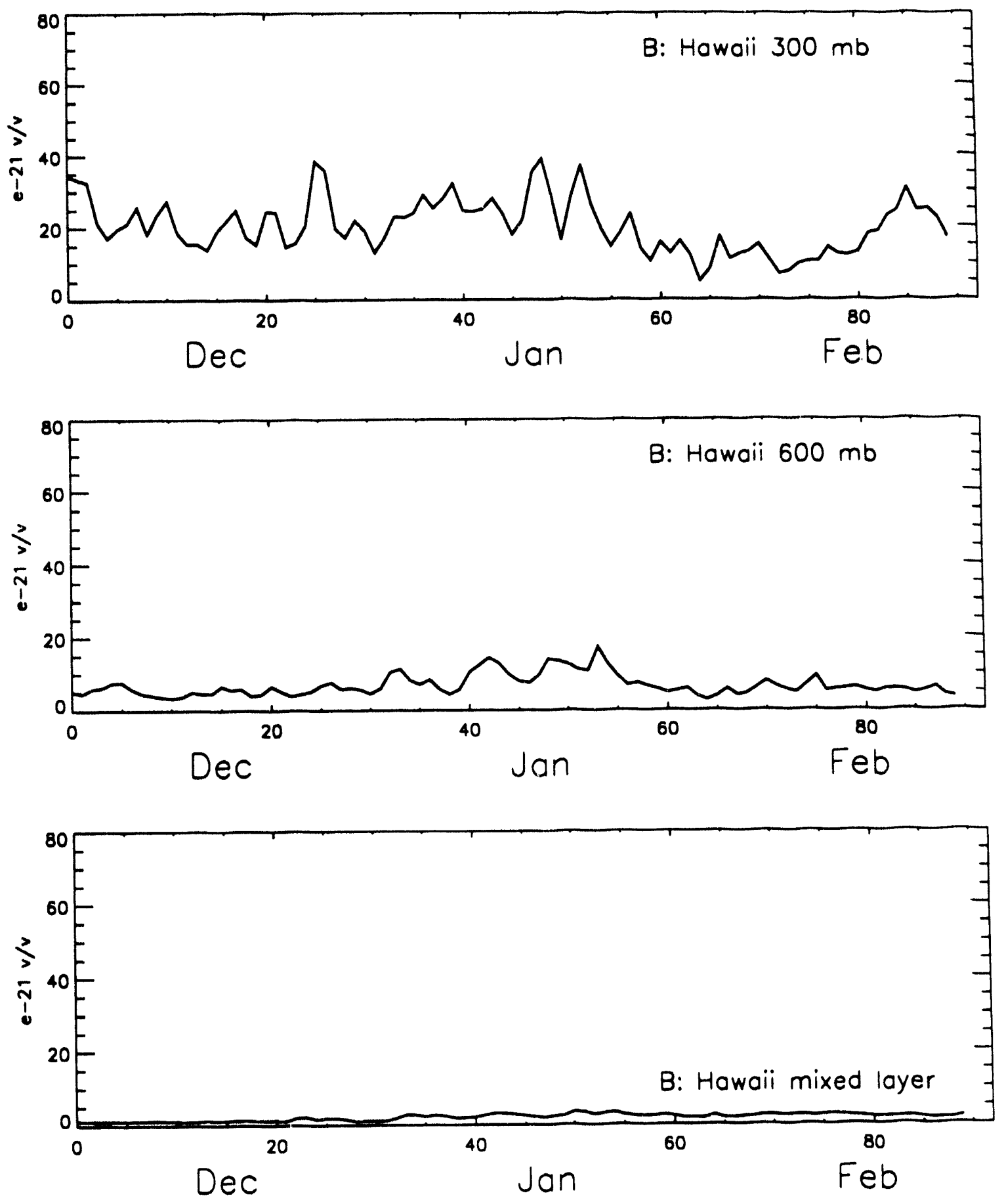
RADON-222 Mixing Ratio
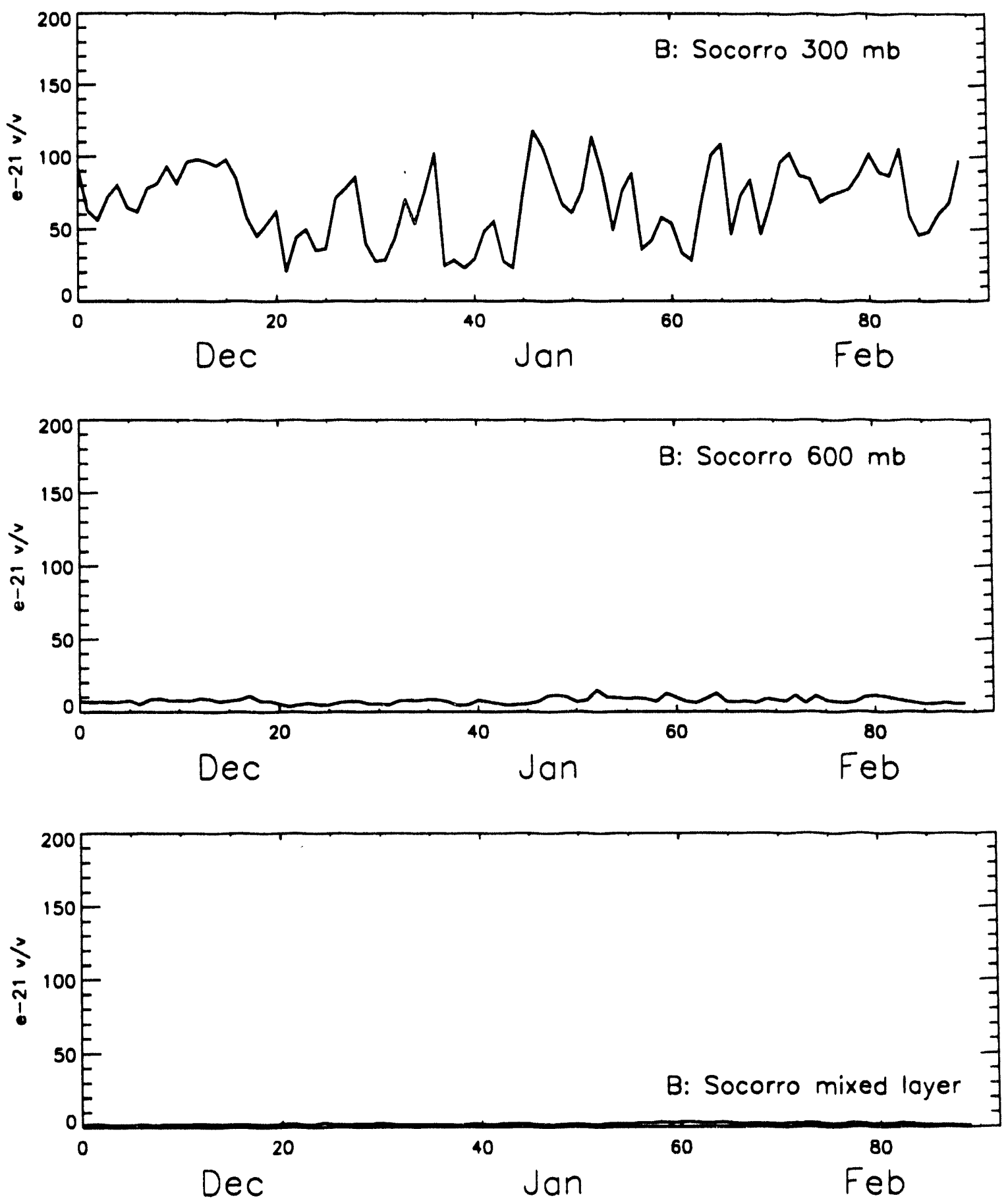
RADON-222 Mixing Ratio
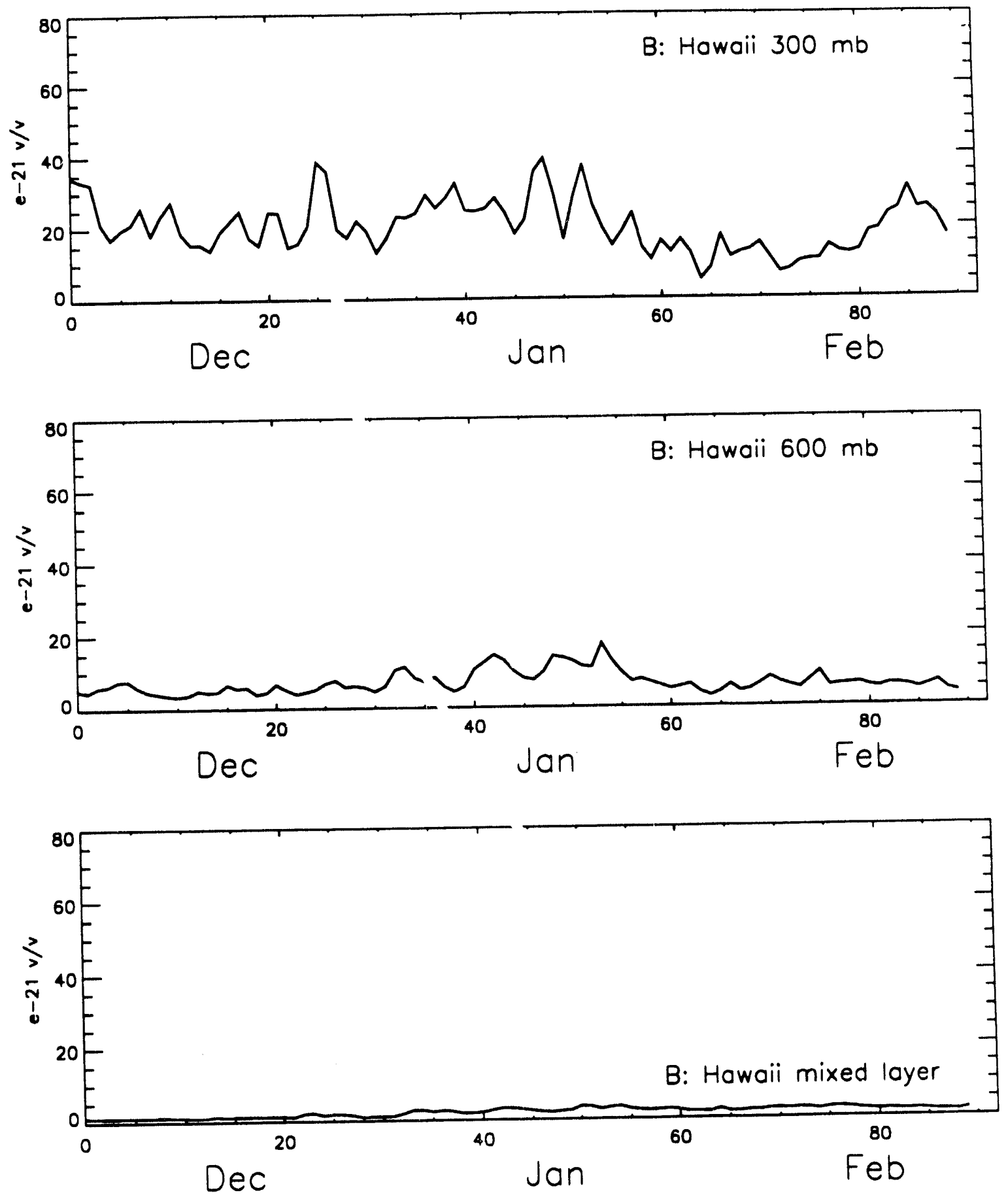


\section{EULERIAN GRANTOUR-LLNL Dec-Jan-Feb}

RADON-222 Mixing Ratio
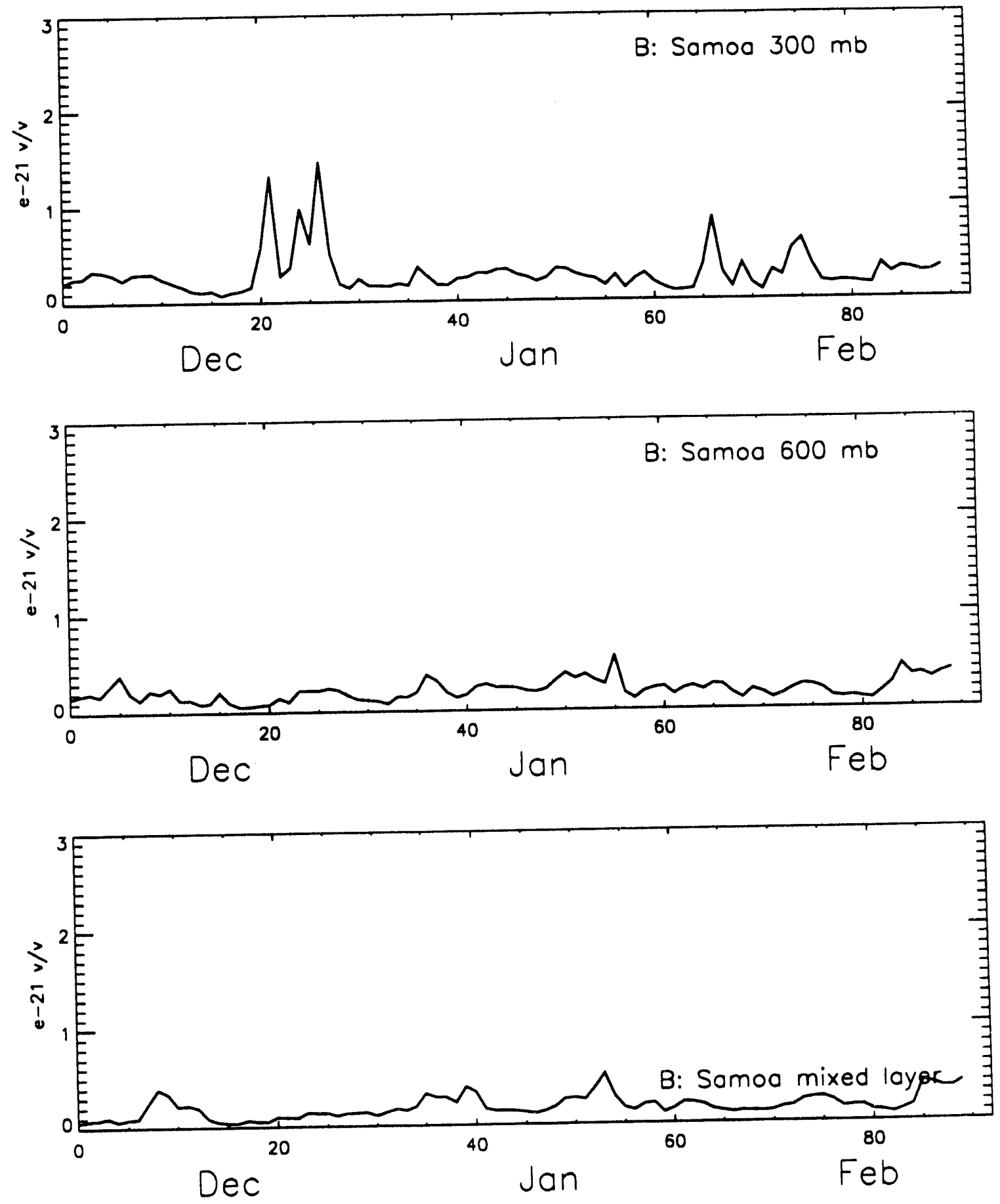
RADON-222 Mixing Ratio
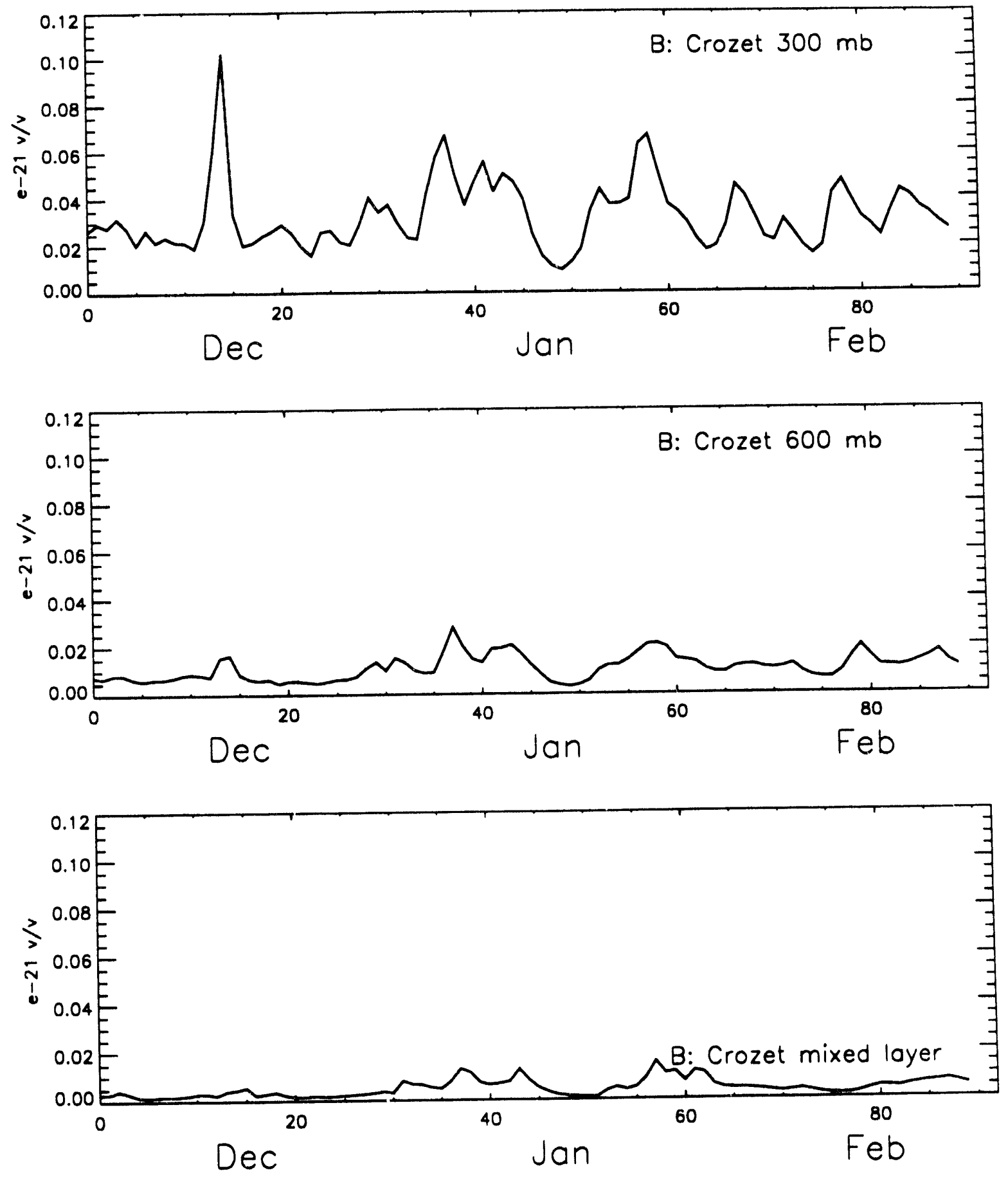
RADON-222 Mixing Ratio
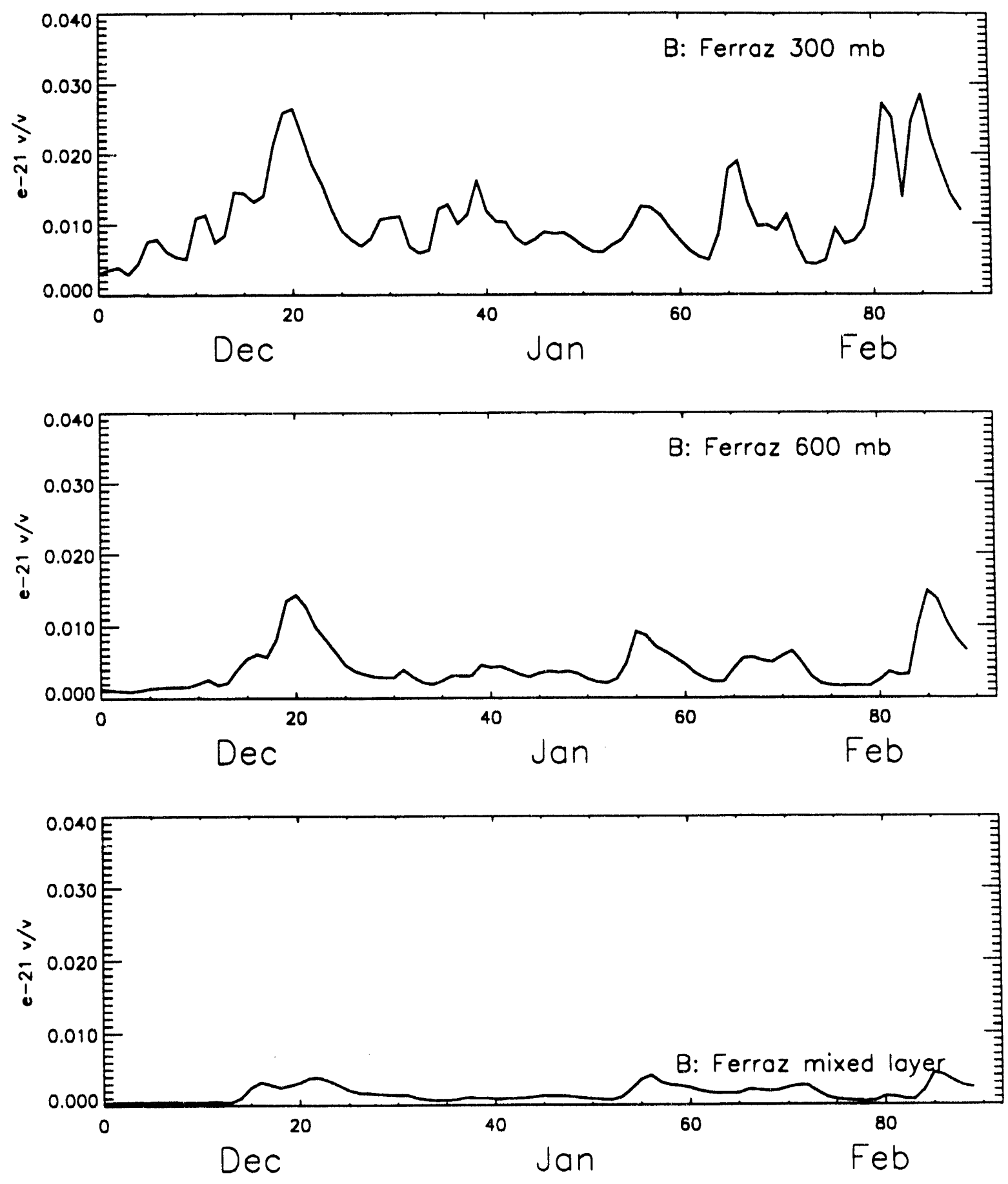


\begin{tabular}{|c|c|c|c|c|c|c|c|c|c|}
\hline \multicolumn{10}{|c|}{ EULERIAN GRANTOUR-LLNL Case B: Time series statistics (E-21 v/v) Dec-Jan-Feb } \\
\hline site & level & mean & stdv & $\operatorname{var}^{\star} \mathrm{E}-42$ & $\min$ & $1 / 4$ & median & $3 / 4$ & $\max$ \\
\hline Kirov & $\begin{array}{c}300 \mathrm{mb} \\
600 \mathrm{mb} \\
\text { mixed } \\
\text { surface }\end{array}$ & \begin{tabular}{r|}
74.65 \\
6.04 \\
1.29 \\
1.25 \\
\end{tabular} & $\begin{array}{r}35.44 \\
2.40 \\
0.50 \\
0.49 \\
\end{array}$ & $\begin{array}{r}1256.58 \\
5.76 \\
0.25 \\
0.24 \\
\end{array}$ & $\begin{array}{r}13.03 \\
2.61 \\
0.56 \\
0.55 \\
\end{array}$ & $\begin{array}{r}45.36 \\
4.24 \\
0.81 \\
0.77 \\
\end{array}$ & $\begin{array}{r}73.38 \\
5.46 \\
1.27 \\
1.24 \\
\end{array}$ & \begin{tabular}{r|}
101.16 \\
7.86 \\
1.65 \\
1.61 \\
\end{tabular} & $\begin{array}{r}159.74 \\
13.81 \\
2.35 \\
2.31 \\
\end{array}$ \\
\hline Cincinnati & $\begin{array}{c}300 \mathrm{mb} \\
600 \mathrm{mb} \\
\text { mixed } \\
\text { surface }\end{array}$ & \begin{tabular}{r|}
218.51 \\
8.02 \\
1.41 \\
1.33 \\
\end{tabular} & $\begin{array}{r}51.16 \\
3.14 \\
0.33 \\
0.31 \\
\end{array}$ & $\begin{array}{r}2617.52 \\
9.87 \\
0.11 \\
0.10 \\
\end{array}$ & \begin{tabular}{r|}
126.25 \\
3.77 \\
0.81 \\
0.78 \\
\end{tabular} & $\begin{array}{r}179.07 \\
5.79 \\
1.15 \\
1.07 \\
\end{array}$ & $\begin{array}{r}214.86 \\
7.24 \\
1.39 \\
1.31 \\
\end{array}$ & $\begin{array}{r}256.54 \\
9.74 \\
1.61 \\
1.54 \\
\end{array}$ & $\begin{array}{r}366.17 \\
19.07 \\
2.50 \\
2.32 \\
\end{array}$ \\
\hline Socorro & $\begin{array}{c}300 \mathrm{mb} \\
600 \mathrm{mb} \\
\text { mixed } \\
\text { surface } \\
\end{array}$ & \begin{tabular}{r|}
67.68 \\
7.46 \\
2.08 \\
1.97 \\
\end{tabular} & $\begin{array}{r}24.84 \\
2.11 \\
0.44 \\
0.42 \\
\end{array}$ & $\begin{array}{r}616.98 \\
4.46 \\
0.19 \\
0.17 \\
\end{array}$ & $\begin{array}{r}20.37 \\
3.49 \\
1.33 \\
1.29 \\
\end{array}$ & $\begin{array}{r}48.12 \\
6.01 \\
1.75 \\
1.66 \\
\end{array}$ & $\begin{array}{r}70.54 \\
7.18 \\
2.02 \\
1.92 \\
\end{array}$ & $\begin{array}{r}86.95 \\
8.66 \\
2.30 \\
2.24 \\
\end{array}$ & $\begin{array}{r}117.80 \\
14.97 \\
3.38 \\
3.25 \\
\end{array}$ \\
\hline Hawaii & $\begin{array}{c}300 \mathrm{mb} \\
600 \mathrm{mb} \\
\text { mixed } \\
\text { surface }\end{array}$ & \begin{tabular}{r|}
20.39 \\
6.75 \\
1.97 \\
1.85 \\
\end{tabular} & $\begin{array}{l}7.55 \\
2.88 \\
0.65 \\
0.60 \\
\end{array}$ & $\begin{array}{r}56.95 \\
8.29 \\
0.42 \\
0.36 \\
\end{array}$ & $\begin{array}{l}5.11 \\
2.76 \\
0.99 \\
0.94 \\
\end{array}$ & $\begin{array}{r}14.64 \\
4.78 \\
1.31 \\
1.23 \\
\end{array}$ & $\begin{array}{r}19.47 \\
5.83 \\
1.95 \\
1.83 \\
\end{array}$ & $\begin{array}{r}24.59 \\
7.60 \\
2.46 \\
2.34 \\
\end{array}$ & $\begin{array}{r}39.04 \\
17.34 \\
3.60 \\
3.19 \\
\end{array}$ \\
\hline Samoa & $\begin{array}{c}300 \mathrm{mb} \\
600 \mathrm{mb} \\
\text { mixed } \\
\text { surface }\end{array}$ & $\begin{array}{l}0.29 \\
0.21 \\
0.18 \\
0.17\end{array}$ & $\begin{array}{l}0.22 \\
0.09 \\
0.10 \\
0.10 \\
\end{array}$ & $\begin{array}{l}0.05 \\
0.01 \\
0.01 \\
0.01 \\
\end{array}$ & $\begin{array}{l}0.0^{-} \\
0.06 \\
0.04 \\
0.04 \\
\end{array}$ & \begin{tabular}{l|}
0.16 \\
0.13 \\
0.11 \\
0.10 \\
\end{tabular} & $\begin{array}{l}0.24 \\
0.20 \\
0.15 \\
0.14 \\
\end{array}$ & $\begin{array}{l}0.31 \\
0.25 \\
0.23 \\
0.21 \\
\end{array}$ & $\begin{array}{l}1.46 \\
0.55 \\
0.51 \\
0.48 \\
\end{array}$ \\
\hline Crozet & $\begin{array}{c}300 \mathrm{mb} \\
600 \mathrm{mb} \\
\text { mixed } \\
\text { surface }\end{array}$ & $\begin{array}{l}3.3 \mathrm{e}-2 \\
1.1 \mathrm{e}-2 \\
4.6 \mathrm{e}-3 \\
4.5 \mathrm{e}-3 \\
\end{array}$ & $\begin{array}{l}1.4 \mathrm{e}-2 \\
4.9 \mathrm{e}-3 \\
3.1 \mathrm{e}-3 \\
3.0 \mathrm{e}-3 \\
\end{array}$ & $\begin{array}{l}2.1 \mathrm{e}-4 \\
2.4 \mathrm{e}-5 \\
9.4 \mathrm{e}-6 \\
9.0 \mathrm{e}-6 \\
\end{array}$ & $\begin{array}{l}9.3 e-3 \\
3.1 \mathrm{e}-3 \\
1.0 \mathrm{e}-3 \\
9.9 \mathrm{e}-4 \\
\end{array}$ & $\begin{array}{l}2.2 \mathrm{e}-2 \\
7.0 \mathrm{e}-3 \\
2.3 \mathrm{e}-3 \\
2.2 \mathrm{e}-3 \\
\end{array}$ & $\begin{array}{l}2.9 \mathrm{e}-2 \\
1.0 \mathrm{e}-2 \\
3.9 \mathrm{e}-3 \\
3.8 \mathrm{e}-3 \\
\end{array}$ & $\begin{array}{l}4.0 \mathrm{e}-2 \\
1.4 \mathrm{e}-2 \\
6.3 \mathrm{e}-3 \\
6.1 \mathrm{e}-3 \\
\end{array}$ & $\begin{array}{l}1.0 e-1 \\
2.8 e-2 \\
1.6 e-2 \\
1.5 e-2 \\
\end{array}$ \\
\hline Ferraz & $\begin{array}{c}300 \mathrm{mb} \\
600 \mathrm{mb} \\
\text { mixed } \\
\text { surface }\end{array}$ & $\begin{array}{l}1.1 \mathrm{e}-2 \\
4.4 \mathrm{e}-3 \\
1.6 \mathrm{e}-3 \\
1.5 \mathrm{e}-3 \\
\end{array}$ & $\begin{array}{l}5.9 e-3 \\
3.2 e-3 \\
1.0 e-3 \\
9.9 e-4 \\
\end{array}$ & $\begin{array}{l}3.5 e-5 \\
1.0 e-5 \\
1.1 e-6 \\
9.8 e-7 \\
\end{array}$ & $\begin{array}{l}2.9 e-3 \\
9.0 e-4 \\
3.0 e-4 \\
2.8 e-4 \\
\end{array}$ & $\begin{array}{l}7.0 \mathrm{e}-3 \\
2.2 \mathrm{e}-3 \\
7.3 \mathrm{e}-4 \\
7.0 \mathrm{e}-4 \\
\end{array}$ & $\begin{array}{l}9.5 \mathrm{e}-3 \\
3.4 \mathrm{e}-3 \\
1.2 \mathrm{e}-3 \\
1.1 \mathrm{e}-3 \\
\end{array}$ & $\begin{array}{l}1.3 \mathrm{e}-2 \\
5.5 \mathrm{e}-3 \\
2.4 \mathrm{e}-3 \\
2.3 \mathrm{e}-3 \\
\end{array}$ & $\begin{array}{l}2.9 e-2 \\
1.5 e-2 \\
4.4 e-3 \\
4.0 e-3 \\
\end{array}$ \\
\hline
\end{tabular}




\section{EULERIAN GRANTOUR-LLNL \\ DJF}

B: Mixed Loyer

RADON-222 Mixing Ratio [*e21 v/v]

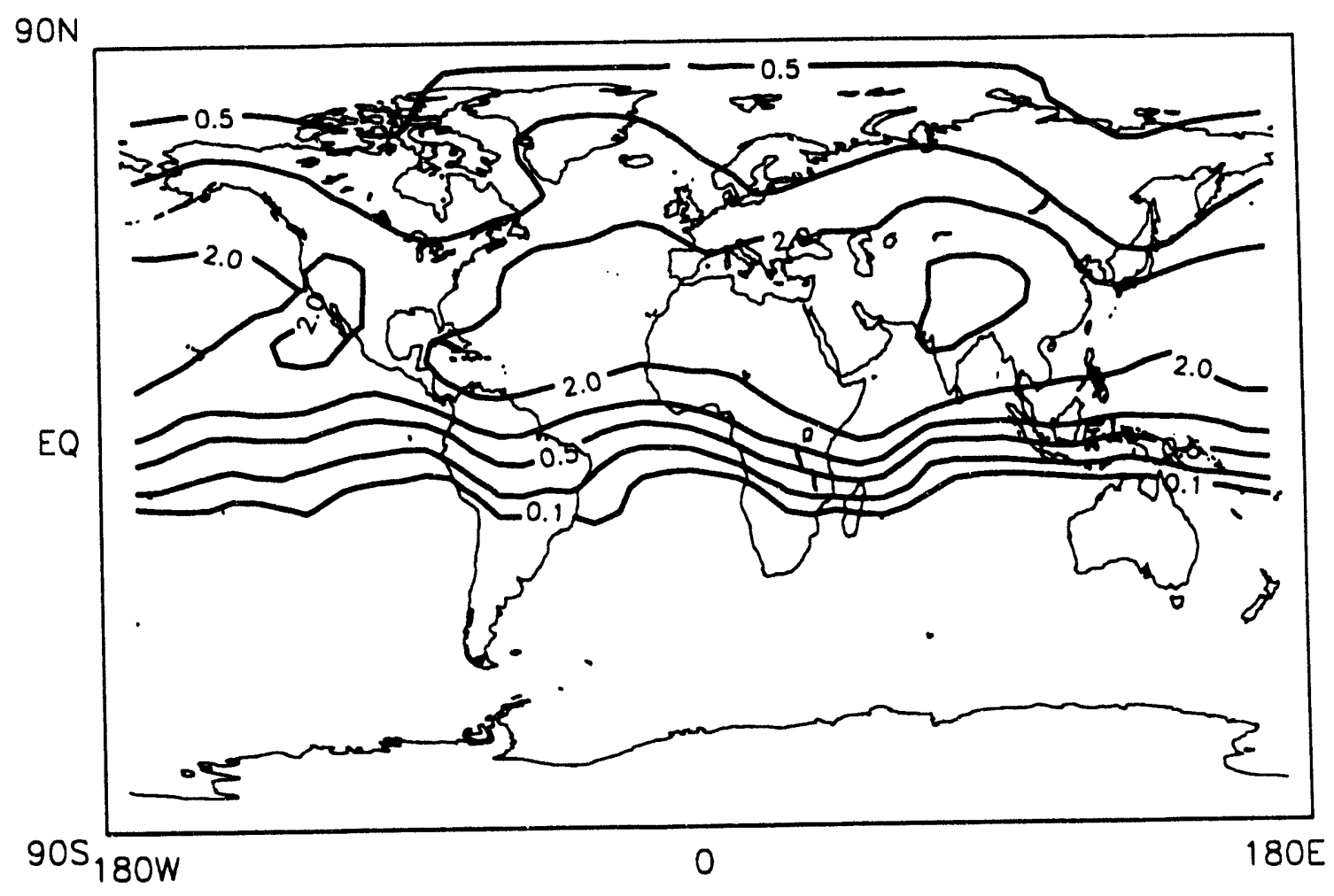


EULERIAN GRANTOUR-LLNL

B: $600 \mathrm{mb}$

RADON-222 Mixing Ratio [*e21 v/v]

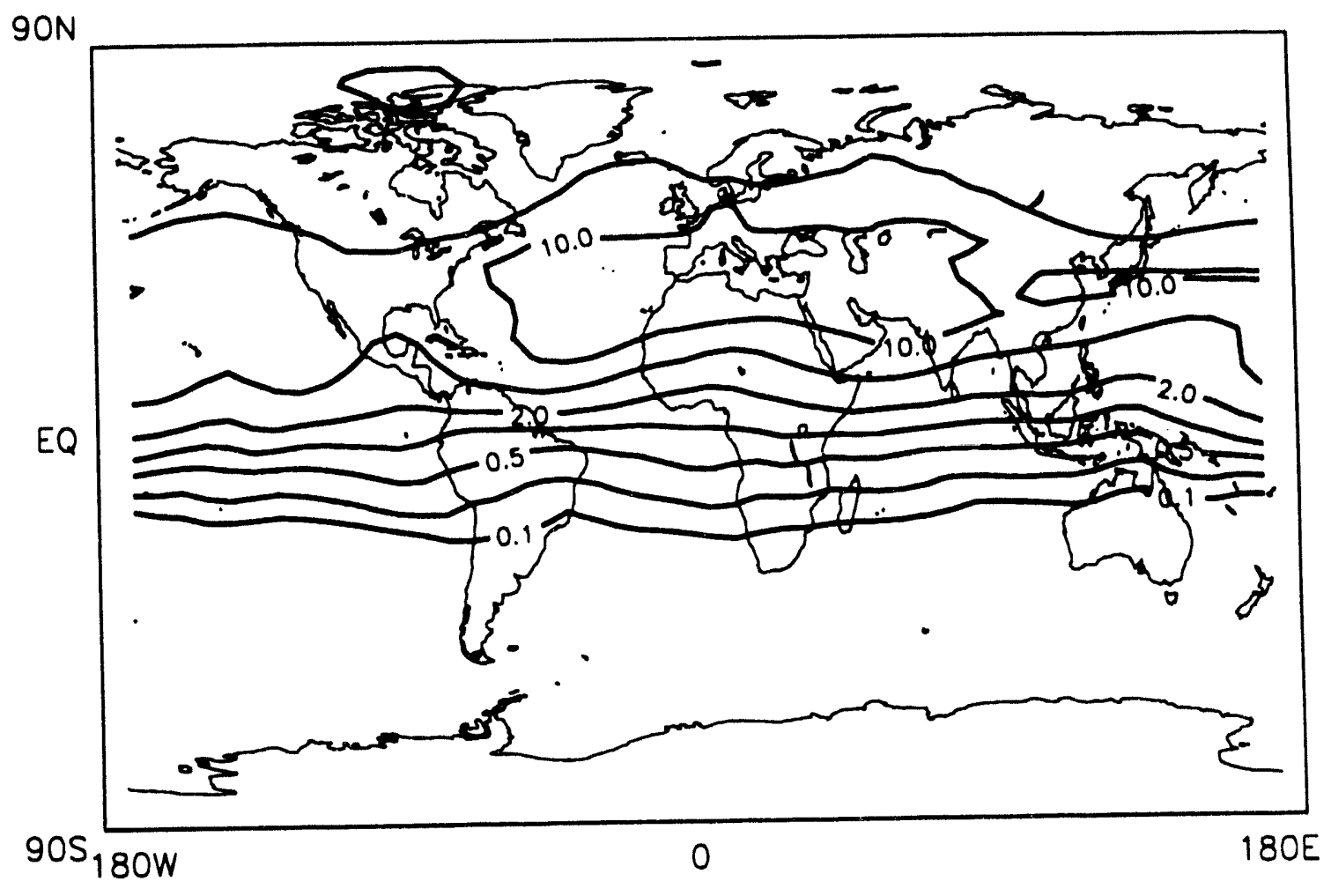



EULERIAN GRANTOUR-LLNL
DJF

\section{B: $300 \mathrm{mb}$}

$$
\text { RADON-222 Mixing Rotio [*e21 v/v] }
$$

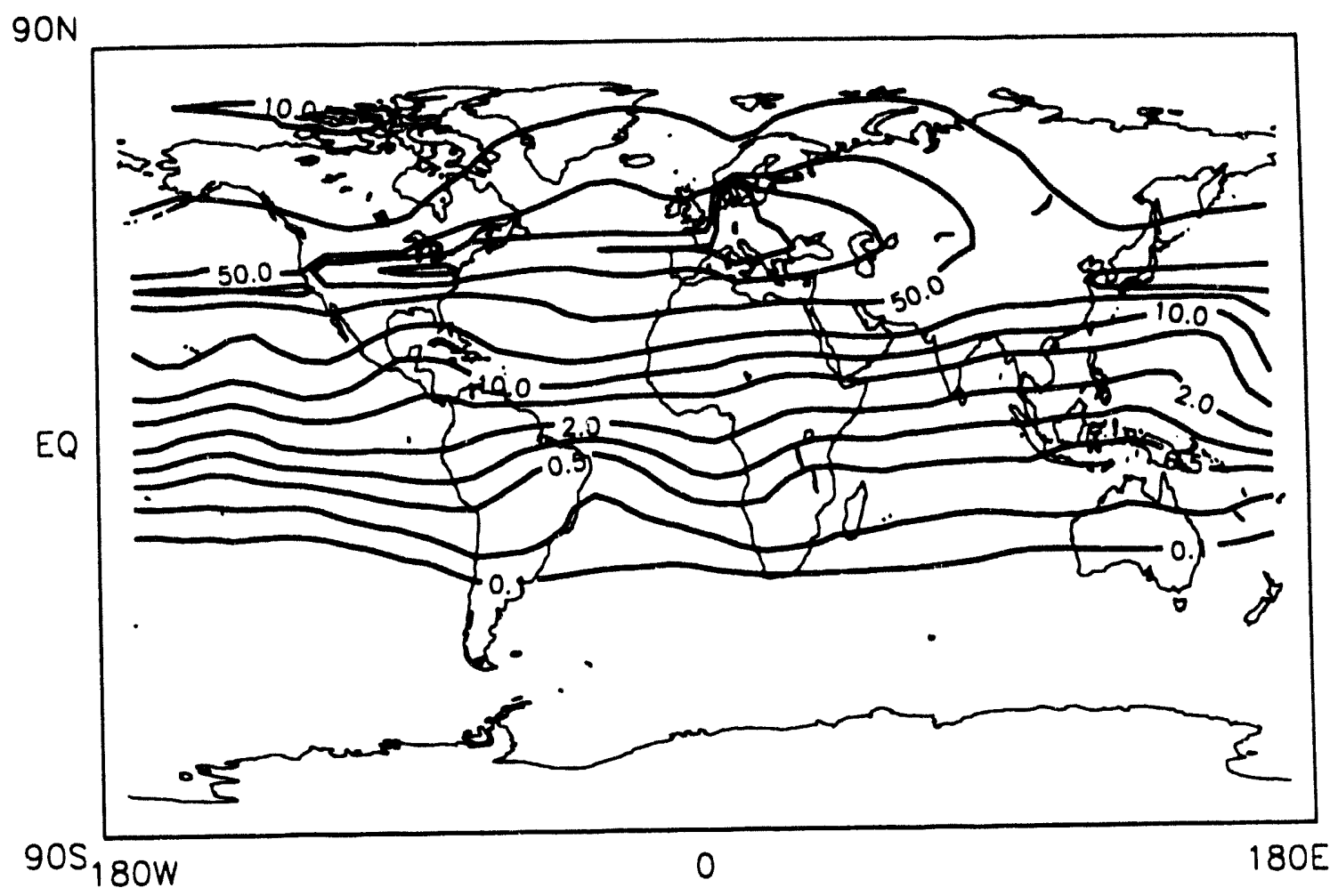


EULERIAN GRANTOUR-LLNL

DJF

B: Zonal Avg

RADON-222 Mixing Rotio [*e21 v/v]

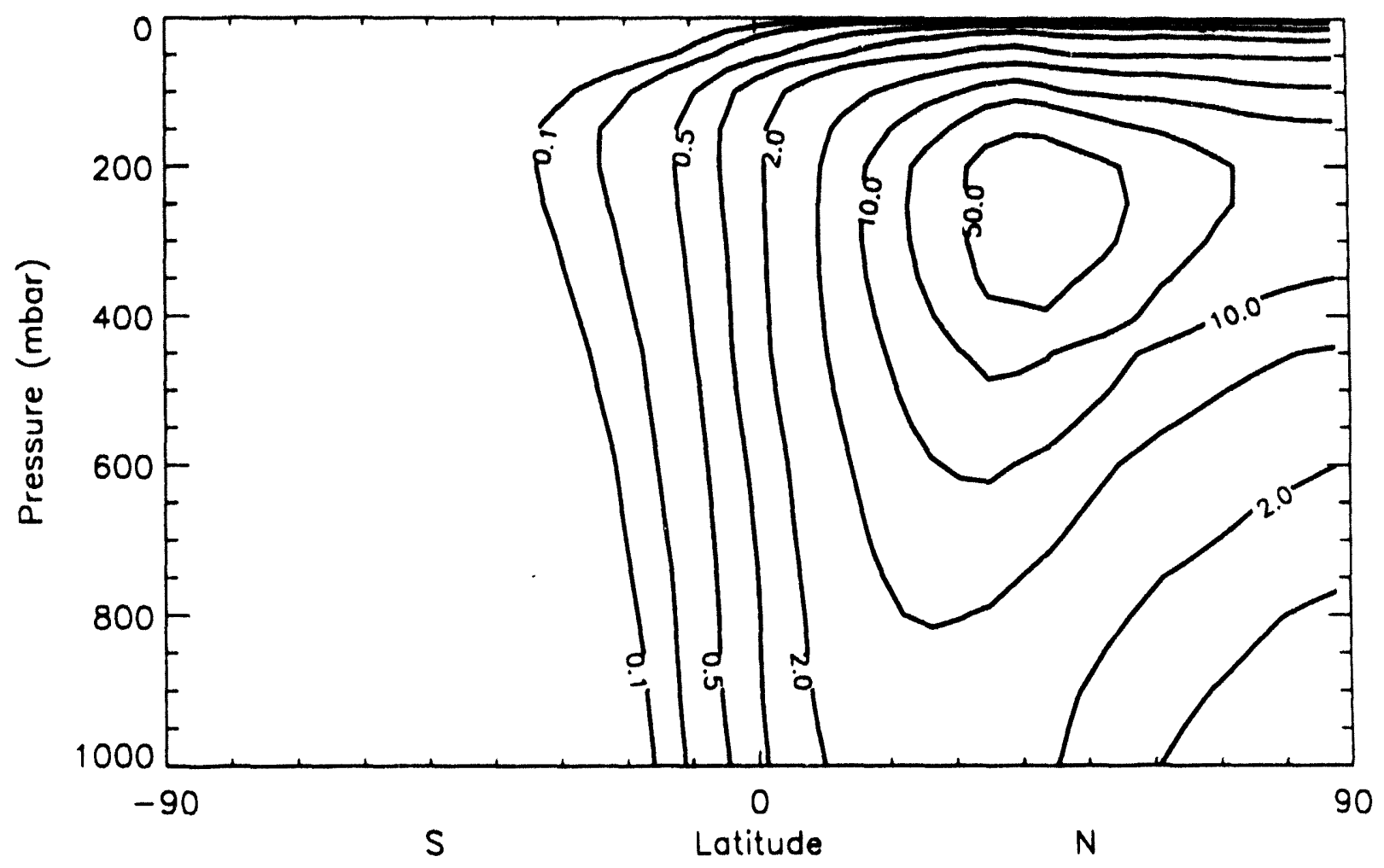


EULERIAN GRANTOUR-LLNL

B: OEW

RADON-222 Mixing Rotio [*e21 v/v]

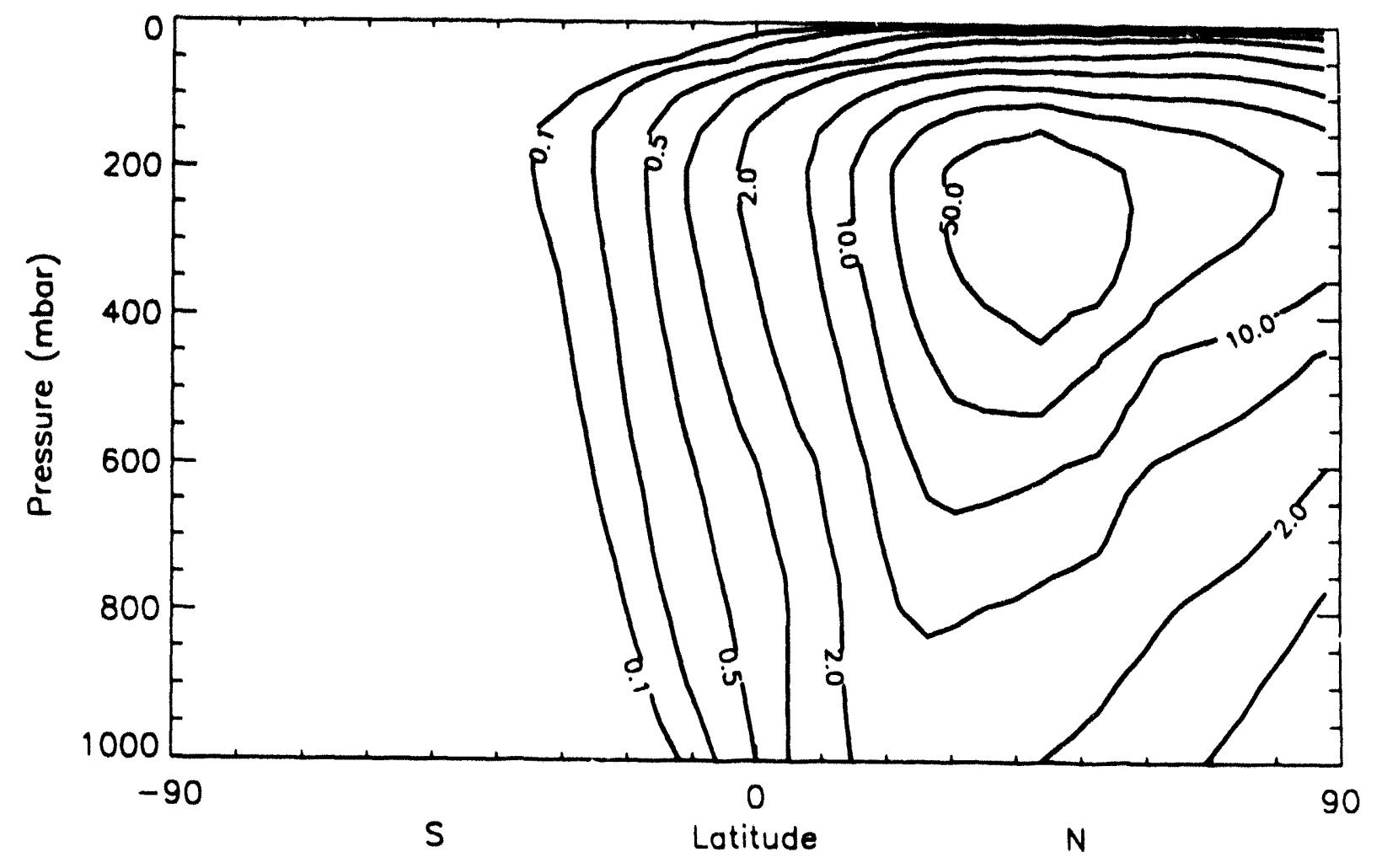


EULERIAN GRANTOUR-LLNL

DJF

B: 180EW

RADON-222 Mixing Rotio [ $* e 21 \mathrm{v} / \mathrm{v}$ ]

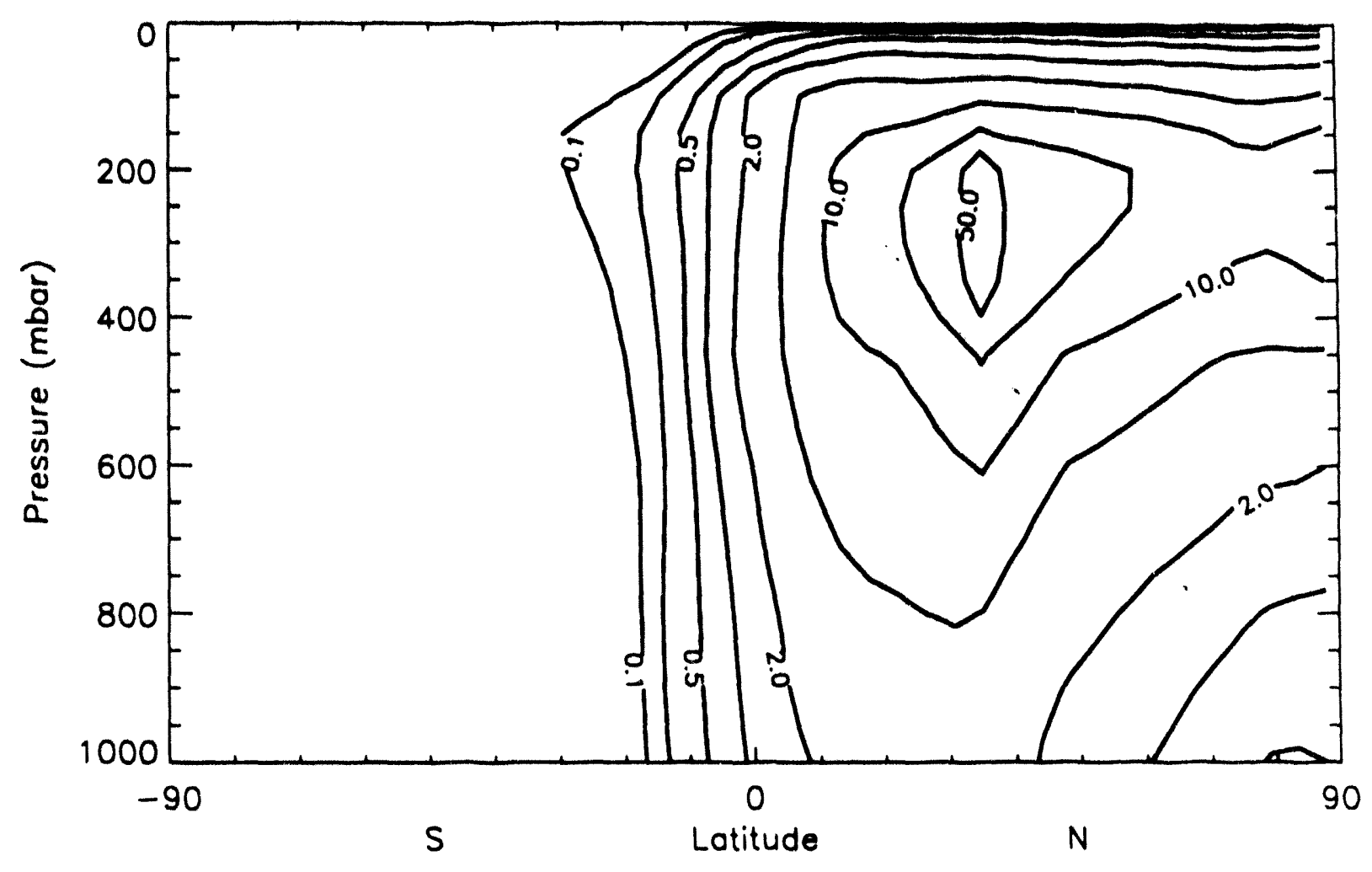


RADON-222 Mixing Ratio
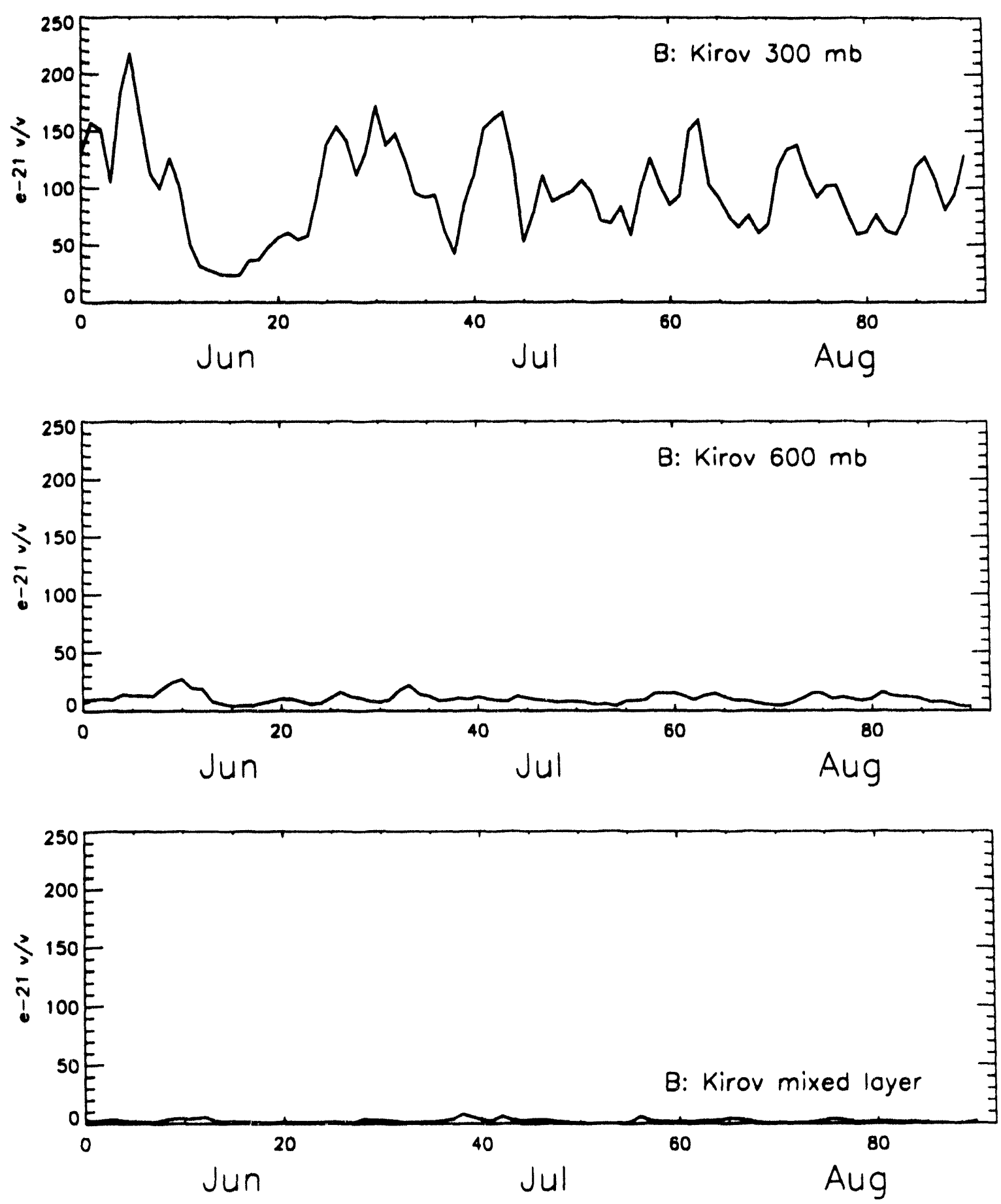
RADON-222 Mixing Ratio
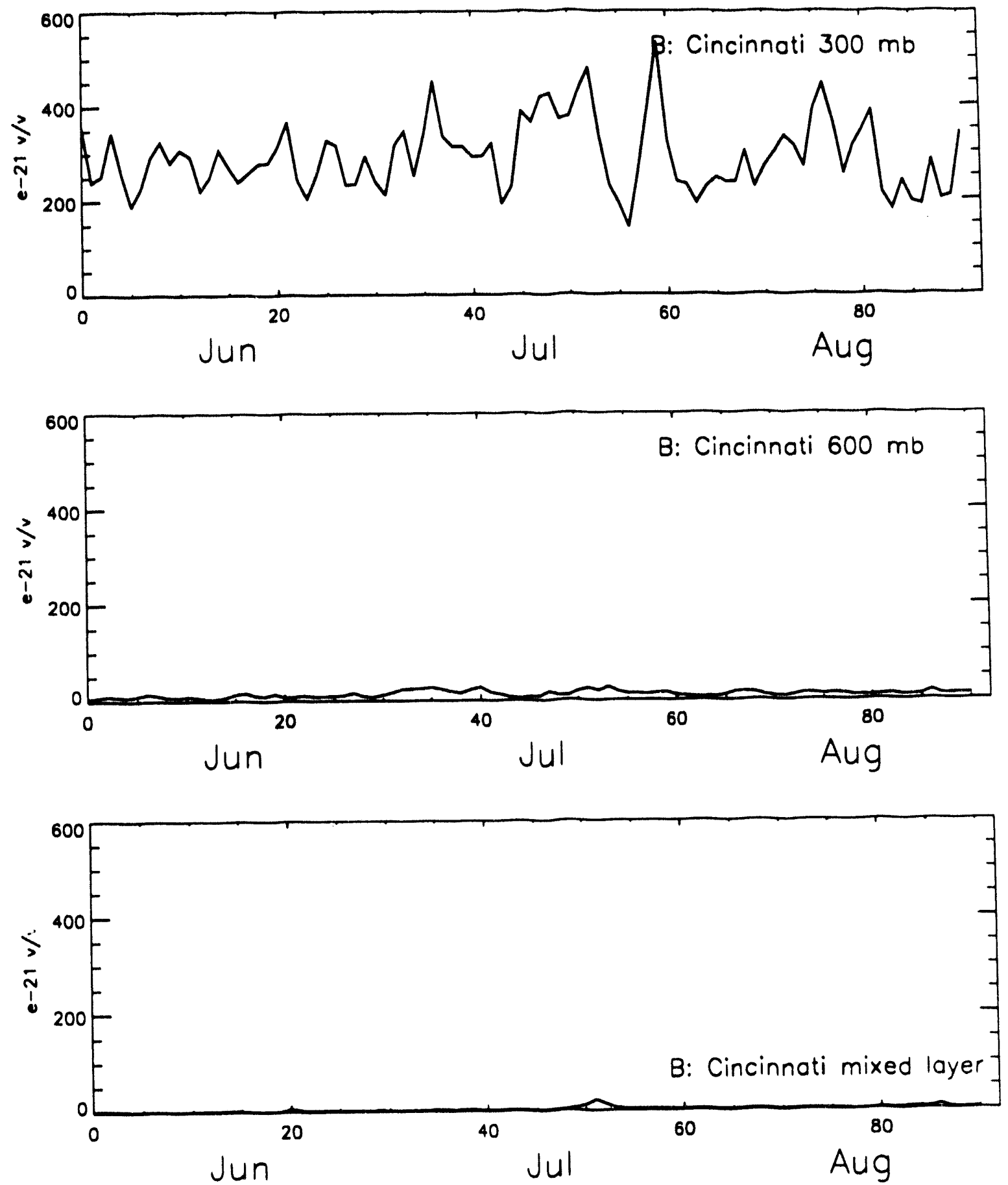


\section{RADON-222 Mixing Ratio}
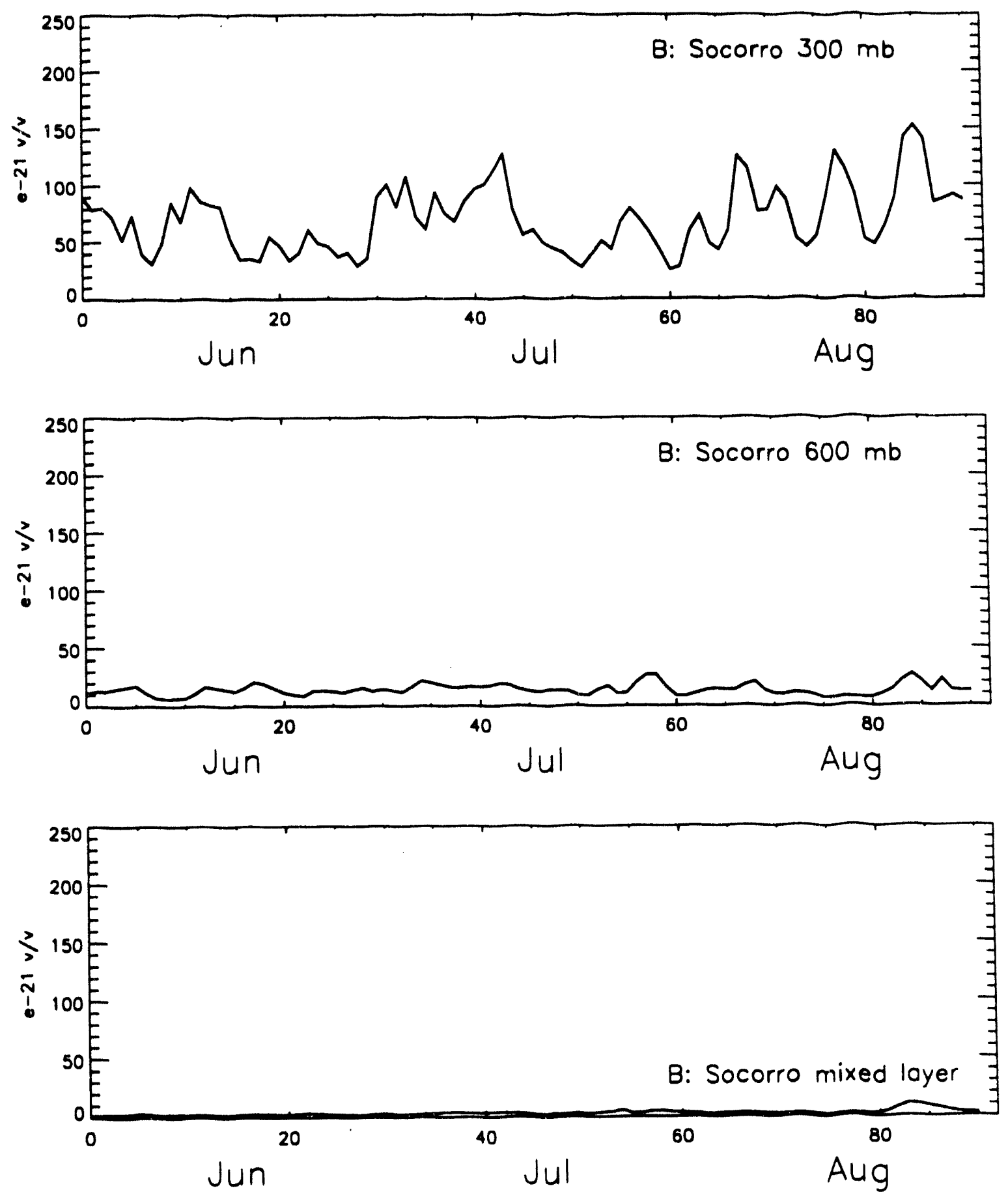
RADON-222 Mixing Ratio
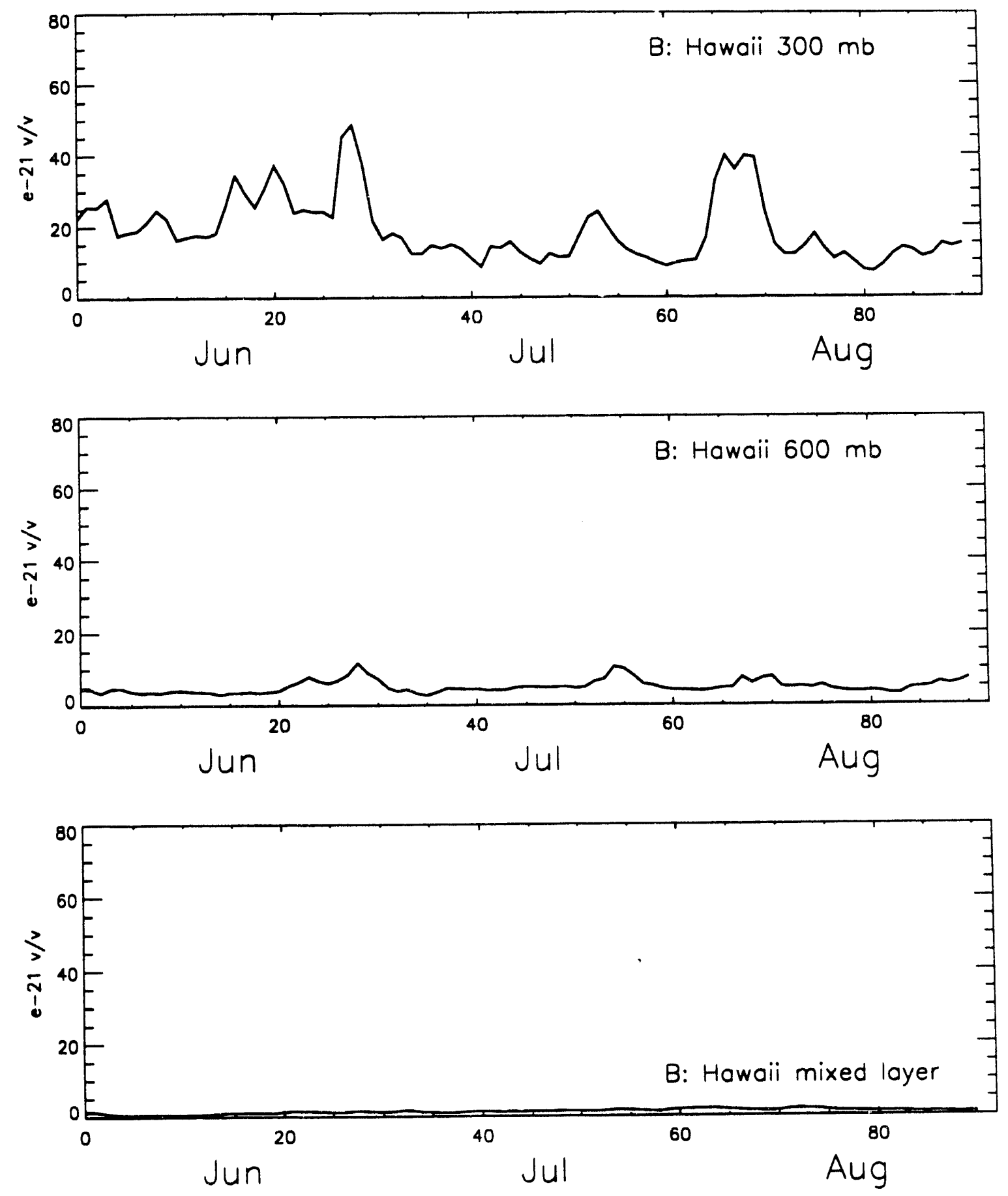


$$
\text { EULERIAN GRANTOUR-LLNL Jun-Jul-Aug }
$$

RADON-222 Mixing Ratio
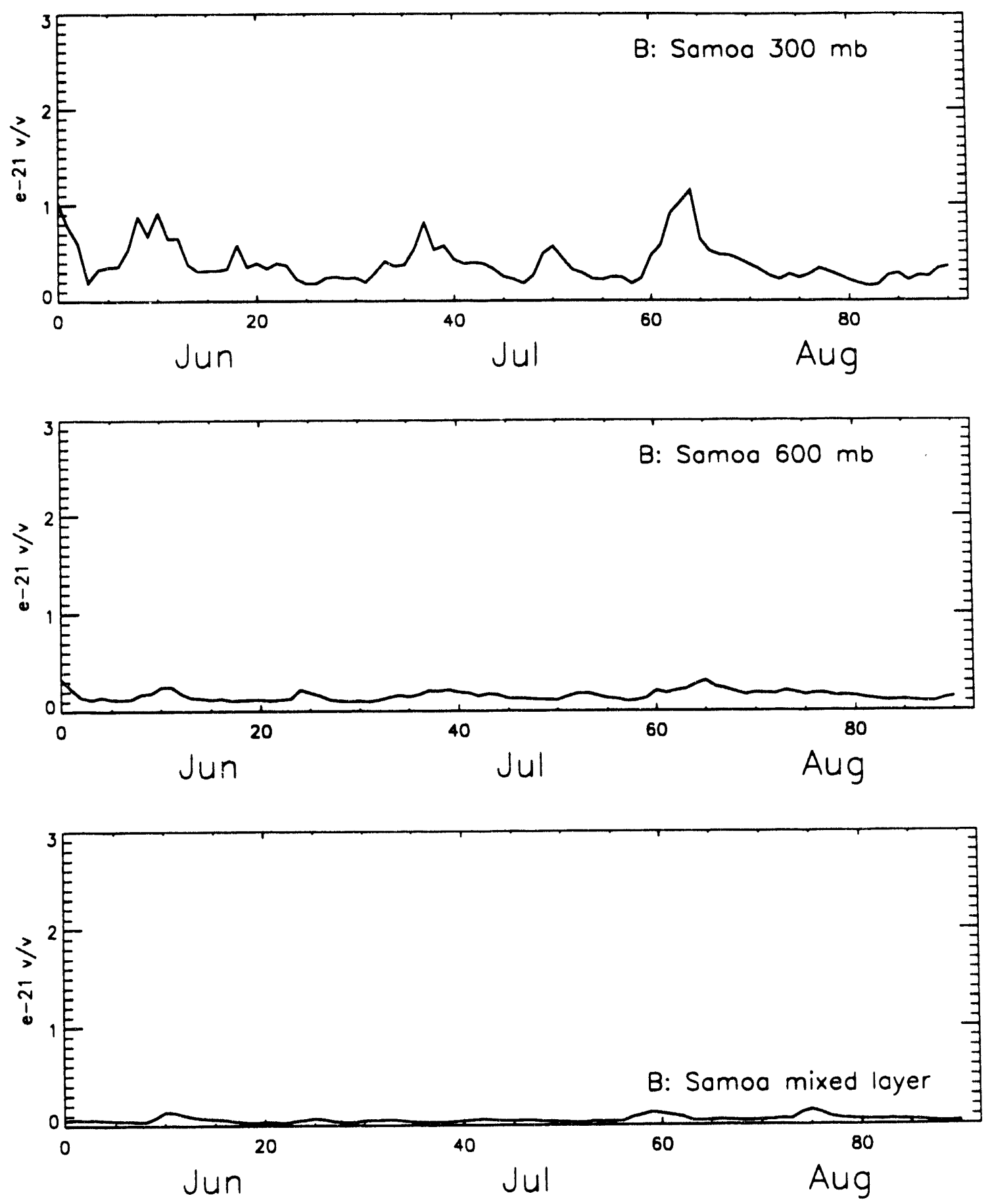
RADON-222 Mixing Ratio
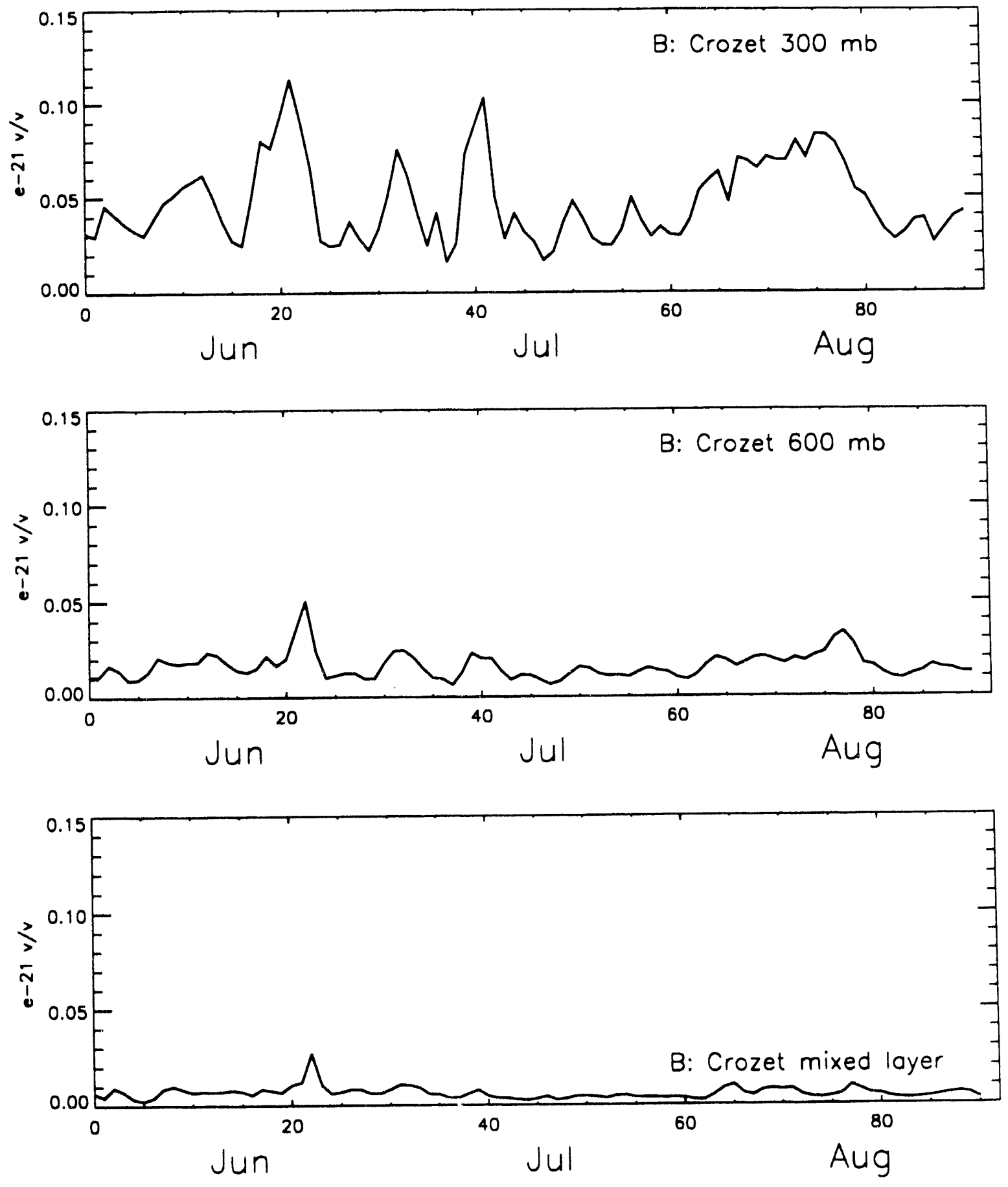
RADON-222 Mixing Ratio
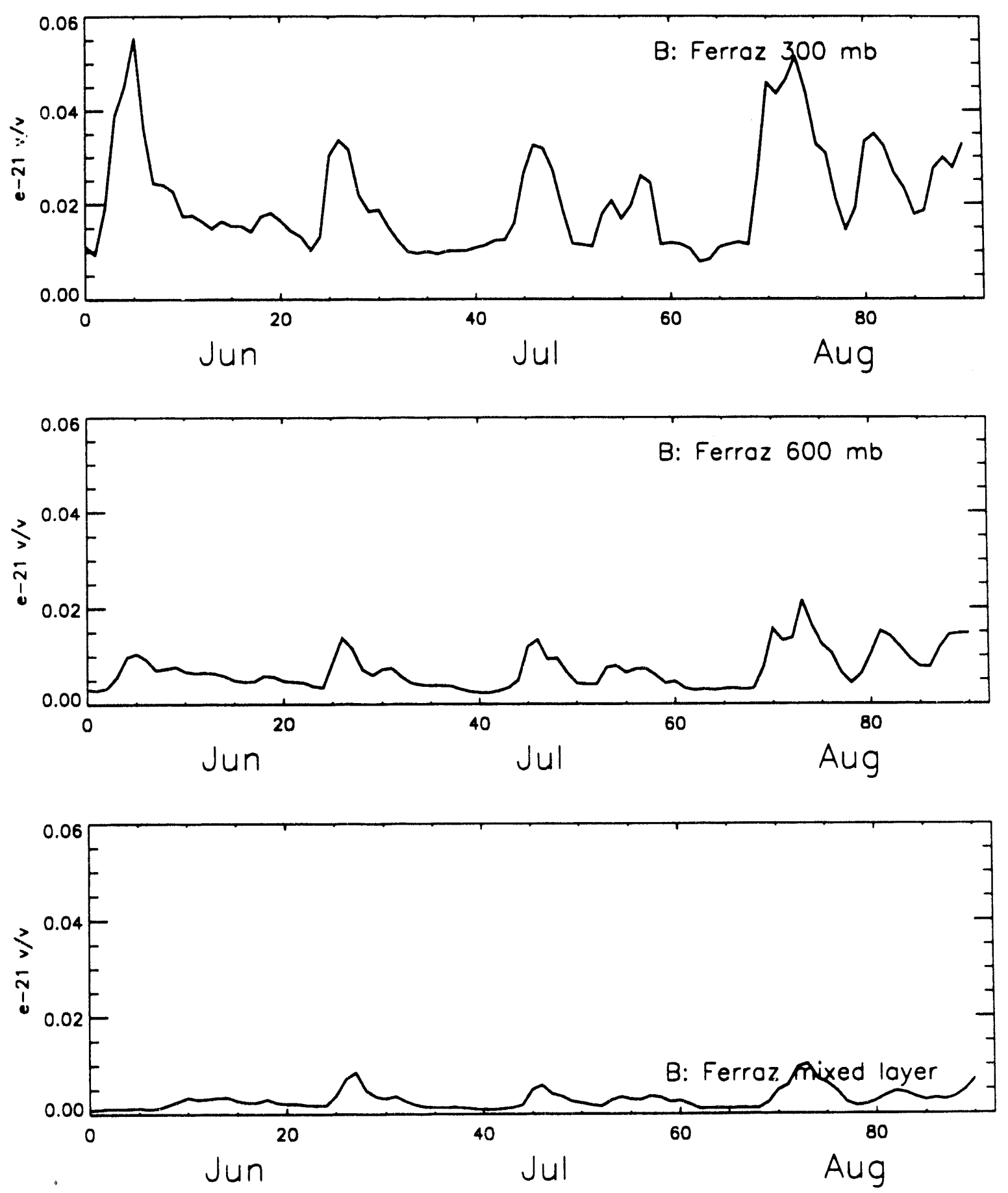


\begin{tabular}{|c|c|c|c|c|c|c|c|c|c|}
\hline \multicolumn{10}{|c|}{ EULERIAN GRANTOUR-LLNL Case B: Time series statistics (E-21 v/v) Jun-Jul-Aug } \\
\hline site & level & mean & stdv & $\operatorname{var}^{\star} E-42$ & $\min$ & $1 / 4$ & median & $3 / 4$ & $\max$ \\
\hline Kirov & $\begin{array}{c}300 \mathrm{mb} \\
600 \mathrm{mb} \\
\text { mixed } \\
\text { surface }\end{array}$ & $\begin{array}{r}97.85 \\
10.49 \\
2.23 \\
1.98 \\
\end{array}$ & $\begin{array}{r}40.08 \\
4.37 \\
1.42 \\
1.14 \\
\end{array}$ & \begin{tabular}{r|}
1606.91 \\
19.06 \\
2.01 \\
1.30 \\
\end{tabular} & \begin{tabular}{r|}
23.54 \\
3.64 \\
0.52 \\
0.51 \\
\end{tabular} & $\begin{array}{r}66.34 \\
7.51 \\
1.19 \\
1.16 \\
\end{array}$ & \begin{tabular}{r|}
95.38 \\
9.80 \\
1.89 \\
1.71 \\
\end{tabular} & \begin{tabular}{r|}
126.08 \\
12.68 \\
2.83 \\
2.58 \\
\end{tabular} & $\begin{array}{r}218.26 \\
26.74 \\
8.09 \\
6.41 \\
\end{array}$ \\
\hline Cincinnati & $\begin{array}{c}300 \mathrm{mb} \\
600 \mathrm{mb} \\
\text { mixed } \\
\text { surface }\end{array}$ & $\begin{array}{r}292.15 \\
12.47 \\
2.79 \\
2.45 \\
\end{array}$ & $\begin{array}{r}74.17 \\
5.58 \\
2.71 \\
1.99 \\
\end{array}$ & $\begin{array}{r}5501.63 \\
31.10 \\
7.36 \\
3.96 \\
\end{array}$ & \begin{tabular}{r|}
142.60 \\
3.89 \\
1.08 \\
1.01 \\
\end{tabular} & \begin{tabular}{r|}
238.65 \\
8.63 \\
1.68 \\
1.61 \\
\end{tabular} & $\begin{array}{r}283.49 \\
10.73 \\
2.24 \\
1.98 \\
\end{array}$ & \begin{tabular}{r|}
335.62 \\
15.39 \\
2.67 \\
2.50 \\
\end{tabular} & $\begin{array}{r}532.77 \\
27.59 \\
22.03 \\
16.11 \\
\end{array}$ \\
\hline Socorro & $\begin{array}{c}300 \mathrm{mb} \\
600 \mathrm{mb} \\
\text { mixed } \\
\text { surface } \\
\end{array}$ & $\begin{array}{r}69.88 \\
13.86 \\
3.16 \\
2.96 \\
\end{array}$ & $\begin{array}{r}29.12 \\
4.61 \\
1.60 \\
1.55 \\
\end{array}$ & $\begin{array}{r}848.13 \\
21.22 \\
2.57 \\
2.40 \\
\end{array}$ & $\begin{array}{r}25.54 \\
6.52 \\
1.92 \\
1.83 \\
\end{array}$ & $\begin{array}{r}46.07 \\
10.74 \\
2.29 \\
2.15 \\
\end{array}$ & \begin{tabular}{r|}
67.99 \\
13.29 \\
2.64 \\
2.47 \\
\end{tabular} & \begin{tabular}{r|}
88.19 \\
16.50 \\
3.33 \\
3.07 \\
\end{tabular} & \begin{tabular}{r|}
151.99 \\
26.68 \\
10.41 \\
9.92 \\
\end{tabular} \\
\hline Hawaii & $\begin{array}{c}300 \mathrm{mb} \\
600 \mathrm{mb} \\
\text { mixed } \\
\text { surface }\end{array}$ & $\begin{array}{r}18.89 \\
5.04 \\
1.27 \\
1.19 \\
\end{array}$ & $\begin{array}{l}9.20 \\
1.73 \\
0.36 \\
0.34 \\
\end{array}$ & $\begin{array}{r}84.66 \\
2.98 \\
0.13 \\
0.12 \\
\end{array}$ & $\begin{array}{l}7.15 \\
2.68 \\
0.54 \\
0.51 \\
\end{array}$ & $\begin{array}{r}11.98 \\
3.85 \\
1.06 \\
1.01 \\
\end{array}$ & $\begin{array}{r}16.23 \\
4.63 \\
1.27 \\
1.24 \\
\end{array}$ & \begin{tabular}{r|}
24.11 \\
5.65 \\
1.46 \\
1.40 \\
\end{tabular} & $\begin{array}{r}48.53 \\
11.71 \\
2.03 \\
1.94 \\
\end{array}$ \\
\hline Samoa & $\begin{array}{c}300 \mathrm{mb} \\
600 \mathrm{mb} \\
\text { mixed } \\
\text { surface }\end{array}$ & $\begin{array}{l}0.40 \\
0.16 \\
0.06 \\
0.05 \\
\end{array}$ & $\begin{array}{l}0.21 \\
0.05 \\
0.03 \\
0.03 \\
\end{array}$ & $\begin{array}{l}0.05 \\
0.00 \\
0.00 \\
0.00 \\
\end{array}$ & $\begin{array}{l}0.15 \\
0.09 \\
0.03 \\
0.02 \\
\end{array}$ & $\begin{array}{l}0.25 \\
0.12 \\
0.04 \\
0.03 \\
\end{array}$ & $\begin{array}{l}0.33 \\
0.15 \\
0.05 \\
0.04 \\
\end{array}$ & $\begin{array}{l}0.48 \\
0.19 \\
0.06 \\
0.06 \\
\end{array}$ & $\begin{array}{l}1.15 \\
0.34 \\
0.15 \\
0.14 \\
\end{array}$ \\
\hline Crozet & $\begin{array}{c}300 \mathrm{mb} \\
600 \mathrm{mb} \\
\text { mixed } \\
\text { surface }\end{array}$ & $\begin{array}{l}4.7 \mathrm{e}-2 \\
1.6 \mathrm{e}-2 \\
6.1 \mathrm{e}-3 \\
5.8 \mathrm{e}-3 \\
\end{array}$ & $\begin{array}{l}2.1 \mathrm{e}-2 \\
6.8 \mathrm{e}-3 \\
3.2 \mathrm{e}-3 \\
3.1 \mathrm{e}-3 \\
\end{array}$ & $\begin{array}{l}4.6 \mathrm{e}-4 \\
4.6 \mathrm{e}-5 \\
1.1 \mathrm{e}-5 \\
9.4 \mathrm{e}-6 \\
\end{array}$ & $\begin{array}{l}1.6 \mathrm{e}-2 \\
6.2 \mathrm{e}-3 \\
2.4 \mathrm{e}-3 \\
2.3 \mathrm{e}-3 \\
\end{array}$ & $\begin{array}{l}3.0 \mathrm{e}-2 \\
1.1 \mathrm{e}-2 \\
3.8 \mathrm{e}-3 \\
3.6 \mathrm{e}-3 \\
\end{array}$ & $\begin{array}{l}4.1 \mathrm{e}-2 \\
1.5 \mathrm{e}-2 \\
5.4 \mathrm{e}-3 \\
5.2 \mathrm{e}-3 \\
\end{array}$ & $\begin{array}{l}6.3 e-2 \\
2.0 e-2 \\
7.6 e-3 \\
7.5 e-3 \\
\end{array}$ & $\begin{array}{l}1.1 \mathrm{e}-1 \\
5.0 \mathrm{e}-2 \\
2.7 \mathrm{e}-2 \\
2.5 \mathrm{e}-2 \\
\end{array}$ \\
\hline Ferraz & $\begin{array}{c}300 \mathrm{mb} \\
600 \mathrm{mb} \\
\text { mixed } \\
\text { surface }\end{array}$ & $\begin{array}{l}2.1 \mathrm{e}-2 \\
7.2 \mathrm{e}-3 \\
2.9 \mathrm{e}-3 \\
2.8 \mathrm{e}-3 \\
\end{array}$ & $\begin{array}{l}1.1 \mathrm{e}-2 \\
4.0 \mathrm{e}-3 \\
1.9 \mathrm{e}-3 \\
1.9 \mathrm{e}-3 \\
\end{array}$ & $\begin{array}{l}1.2 \mathrm{e}-4 \\
1.6 \mathrm{e}-5 \\
3.8 \mathrm{e}-6 \\
3.5 \mathrm{e}-6 \\
\end{array}$ & $\begin{array}{l}7.8 e-3 \\
2.4 e-3 \\
8.8 e-4 \\
6.9 e-4 \\
\end{array}$ & $\begin{array}{l}1.1 \mathrm{e}-2 \\
4.1 \mathrm{e}-3 \\
1.4 \mathrm{e}-3 \\
1.3 \mathrm{e}-3 \\
\end{array}$ & $\begin{array}{l}1.8 \mathrm{e}-2 \\
6.4 \mathrm{e}-3 \\
2.6 \mathrm{e}-3 \\
2.5 \mathrm{e}-3 \\
\end{array}$ & $\begin{array}{l}2.8 \mathrm{e}-2 \\
9.4 \mathrm{e}-3 \\
3.6 \mathrm{e}-3 \\
3.4 \mathrm{e}-3 \\
\end{array}$ & $\begin{array}{l}5.6 \mathrm{e}-2 \\
2.2 \mathrm{e}-2 \\
1.0 \mathrm{e}-2 \\
1.0 \mathrm{e}-2 \\
\end{array}$ \\
\hline
\end{tabular}


EULERIAN GRANTOUR-LLNL

JJA

B: Mixed Loyer

RADON-222 Mixing Ratio [*e21 v/v]

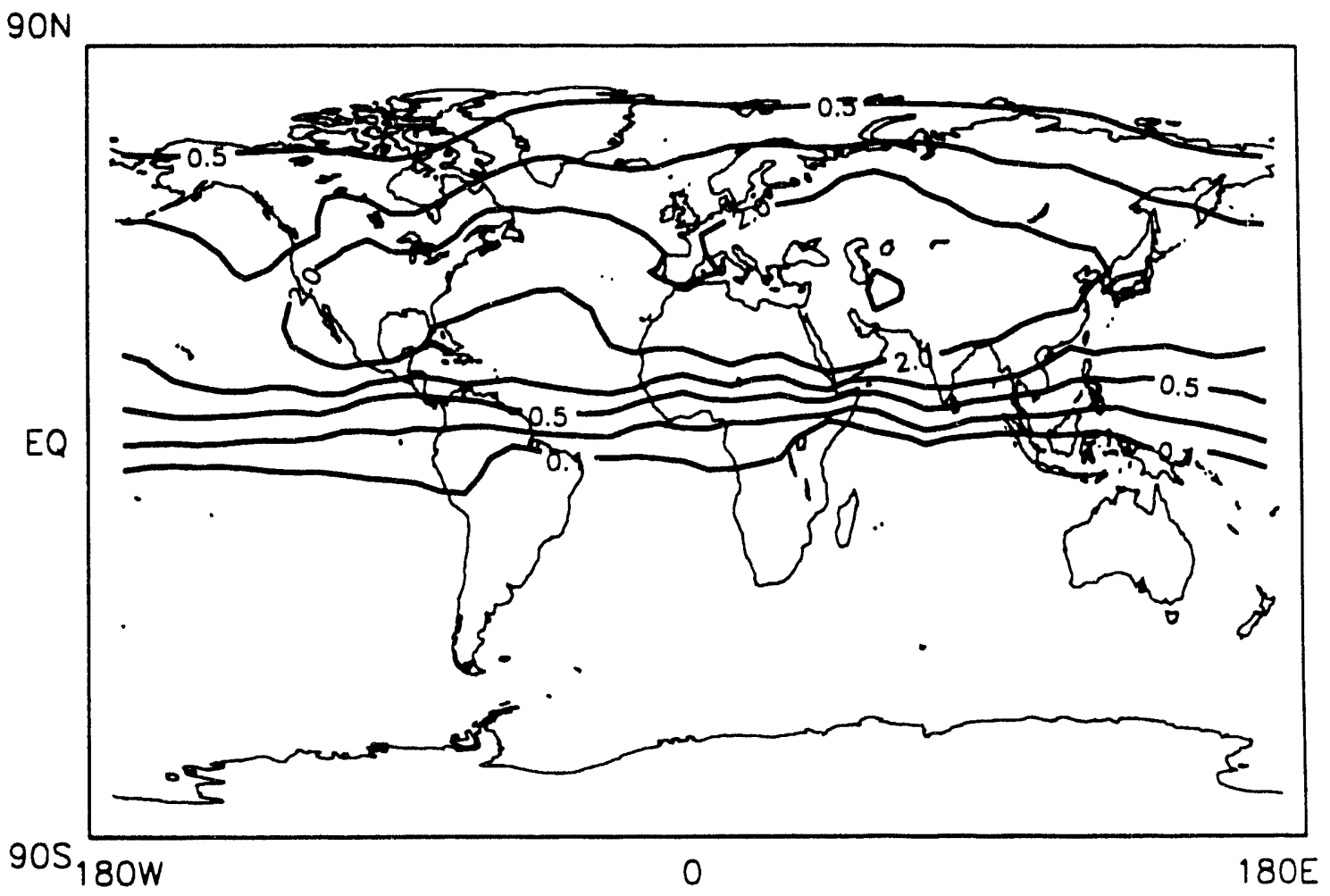


EULERIAN GRANTOUR-LLNL JJA

B: $600 \mathrm{mb}$

RADON-222 Mixing Ratio $[* e 21 \mathrm{v} / \mathrm{v}]$

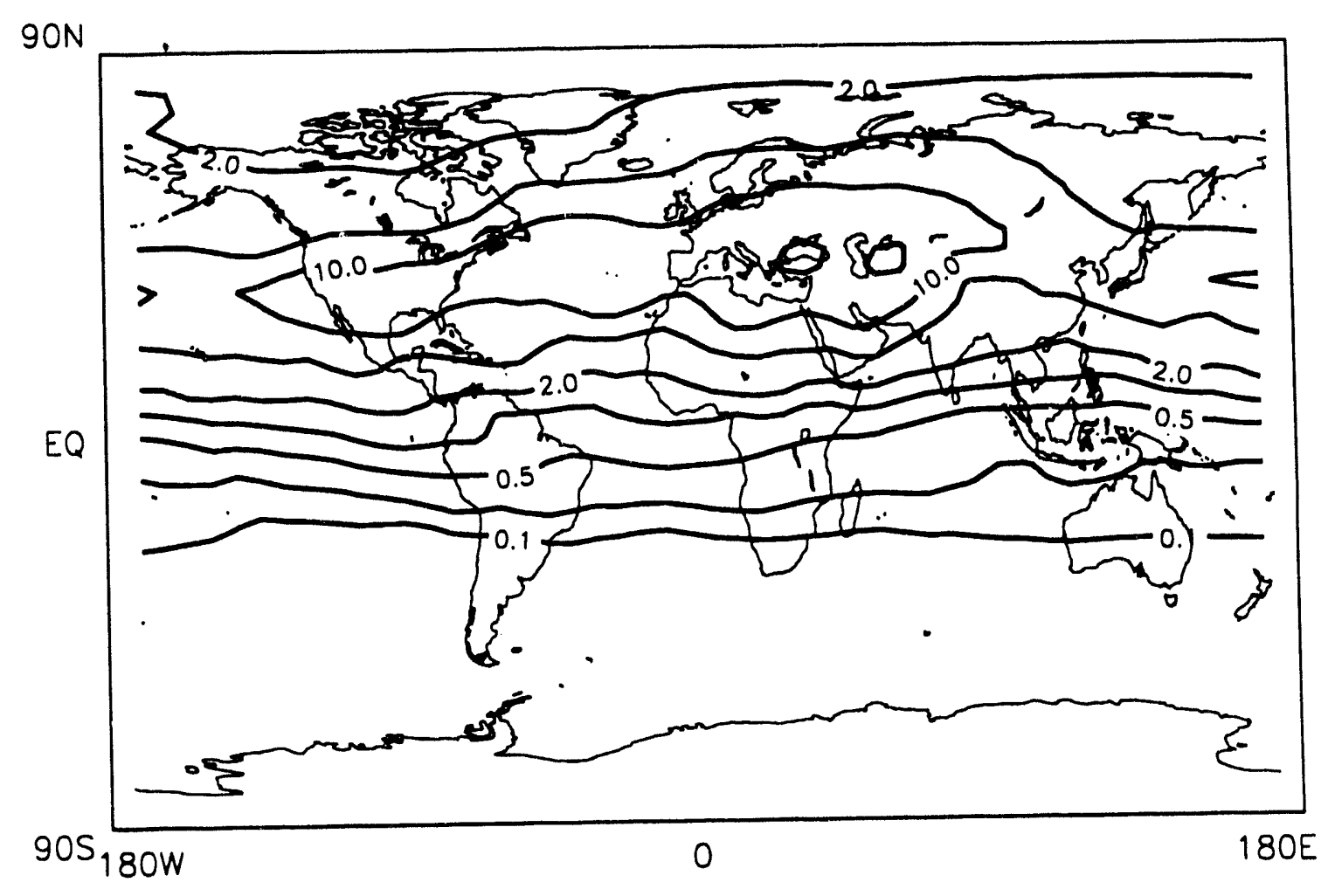


EULERIAN GRANTOUR - LLNL JJA

B: $300 \mathrm{mb}$

RADON-222 Mixing Ratio [*e21 v/v]

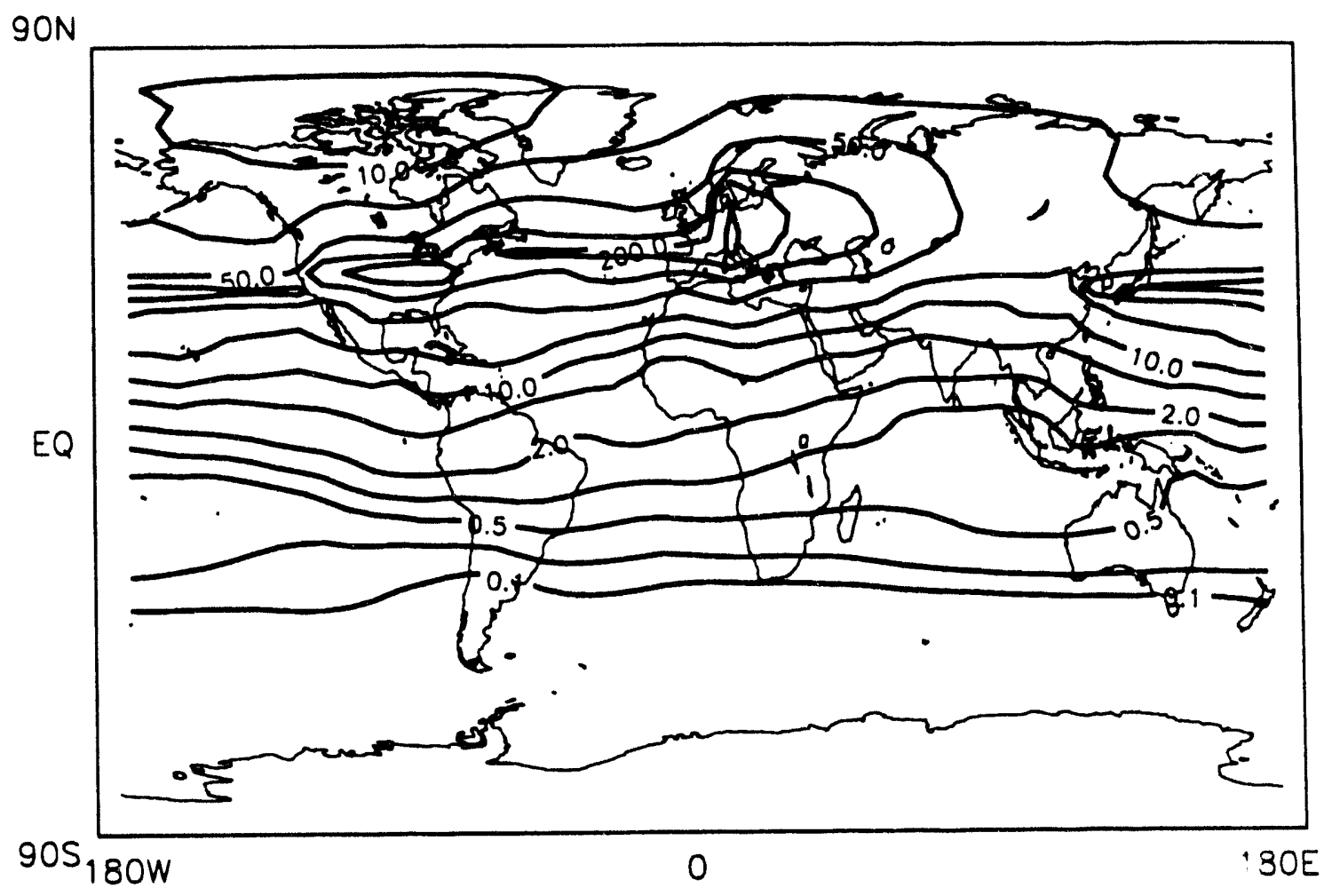




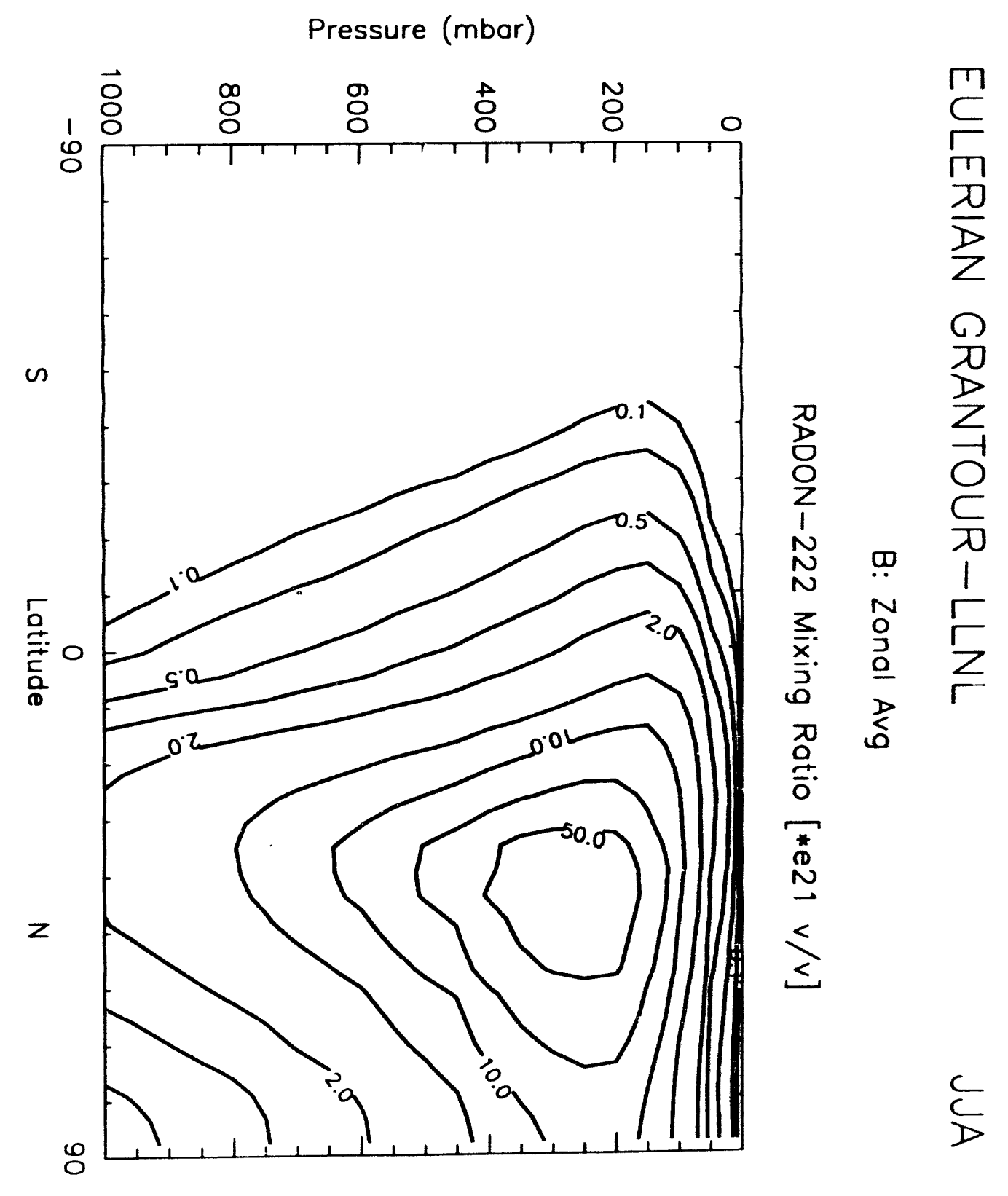




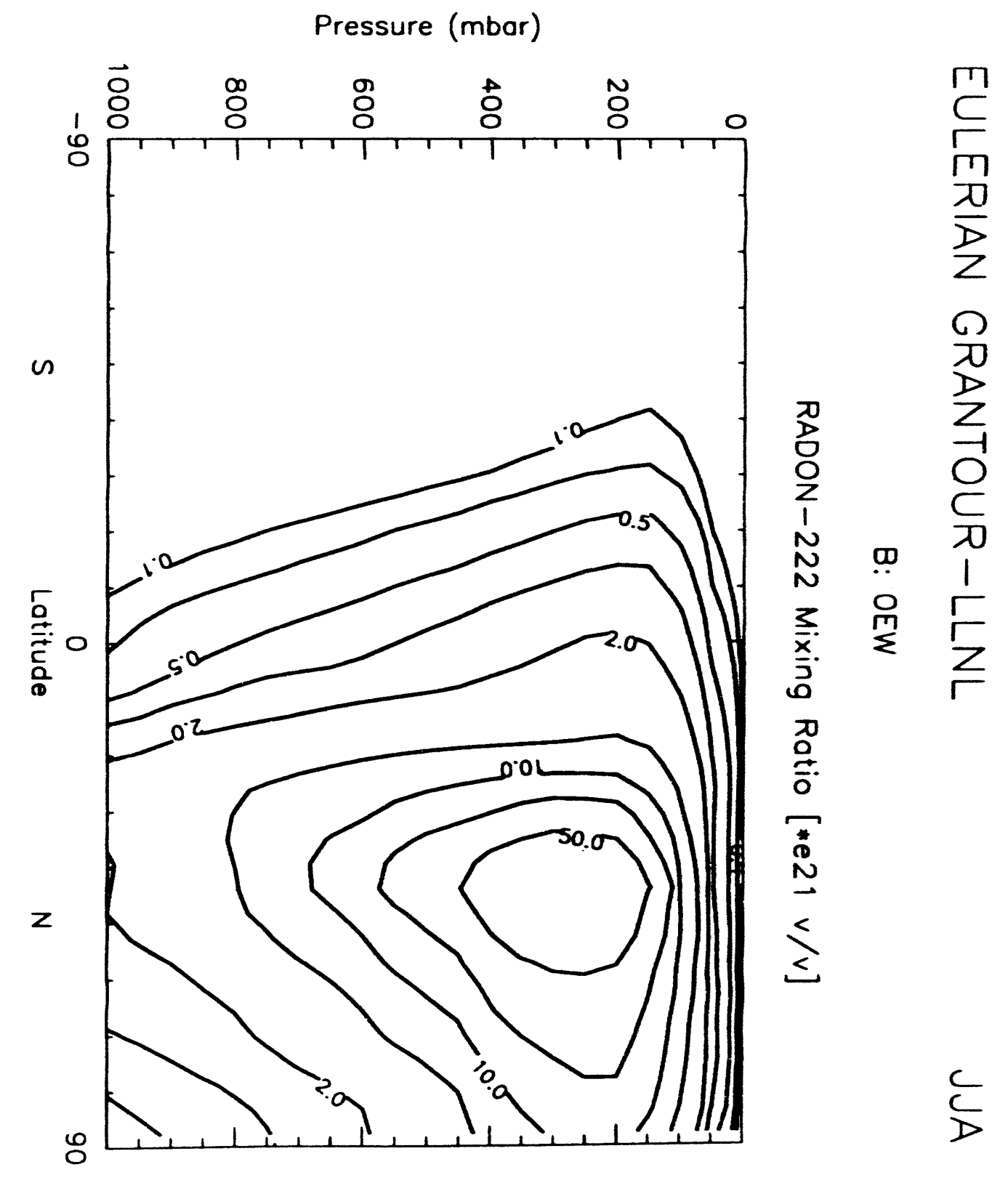


EULERIAN GRANTOUR-LLNL

B: $180 E W$

RADON-222 Mixing Ratio [*e21 v/v]

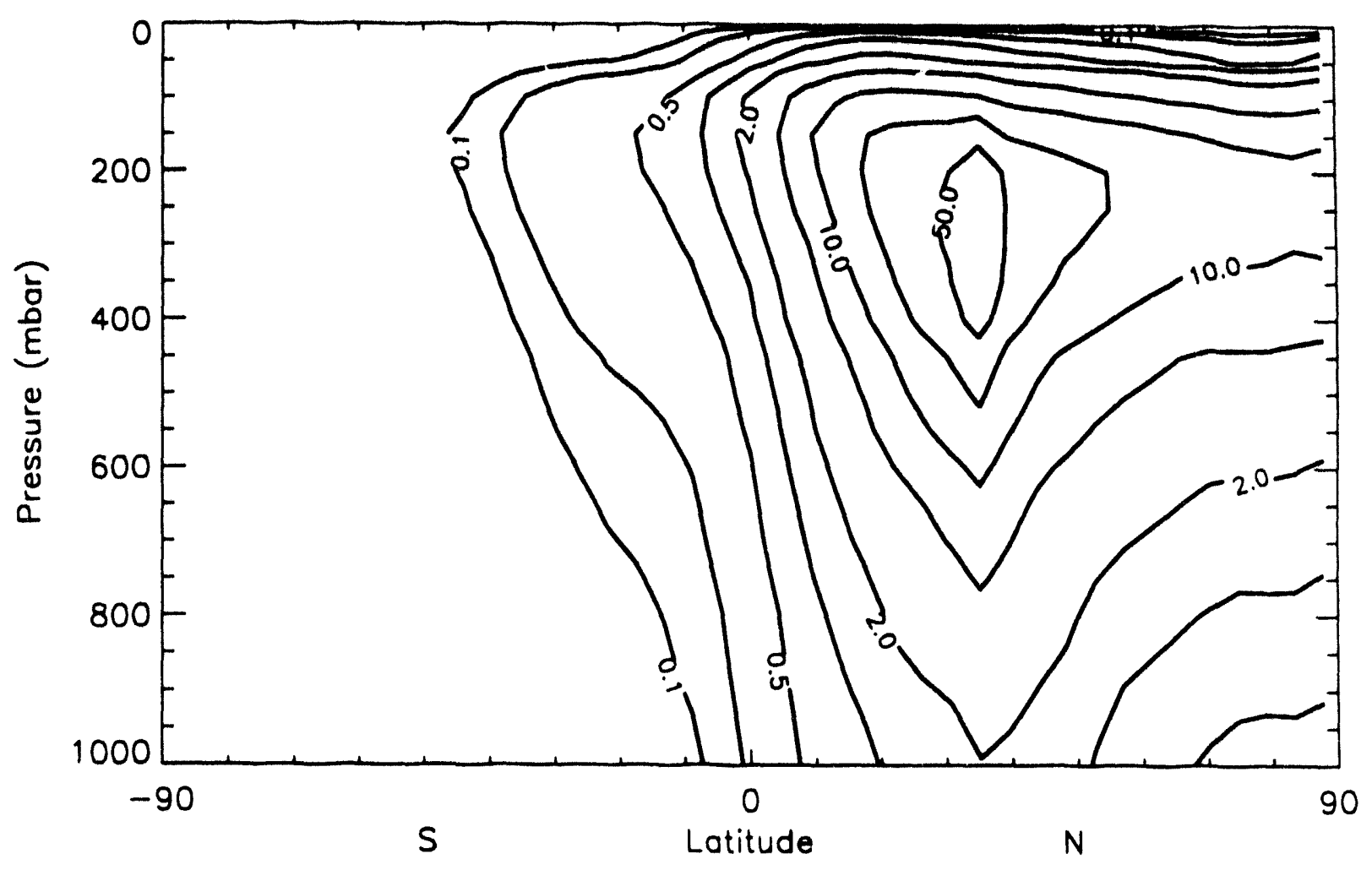


Case C: Lightning 
RADON-222 Mixing Ratio
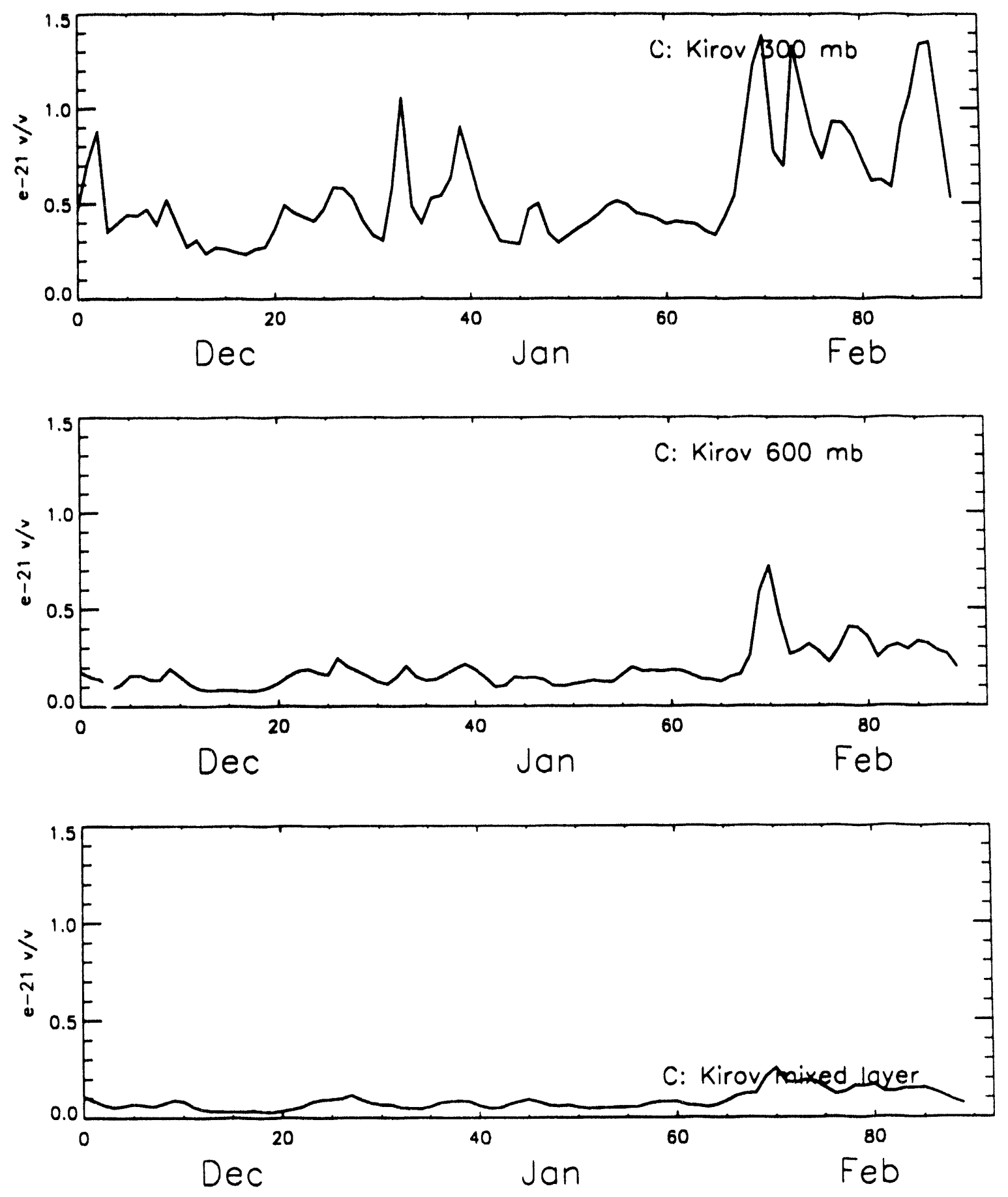
RADON-222 Mixing Ratio
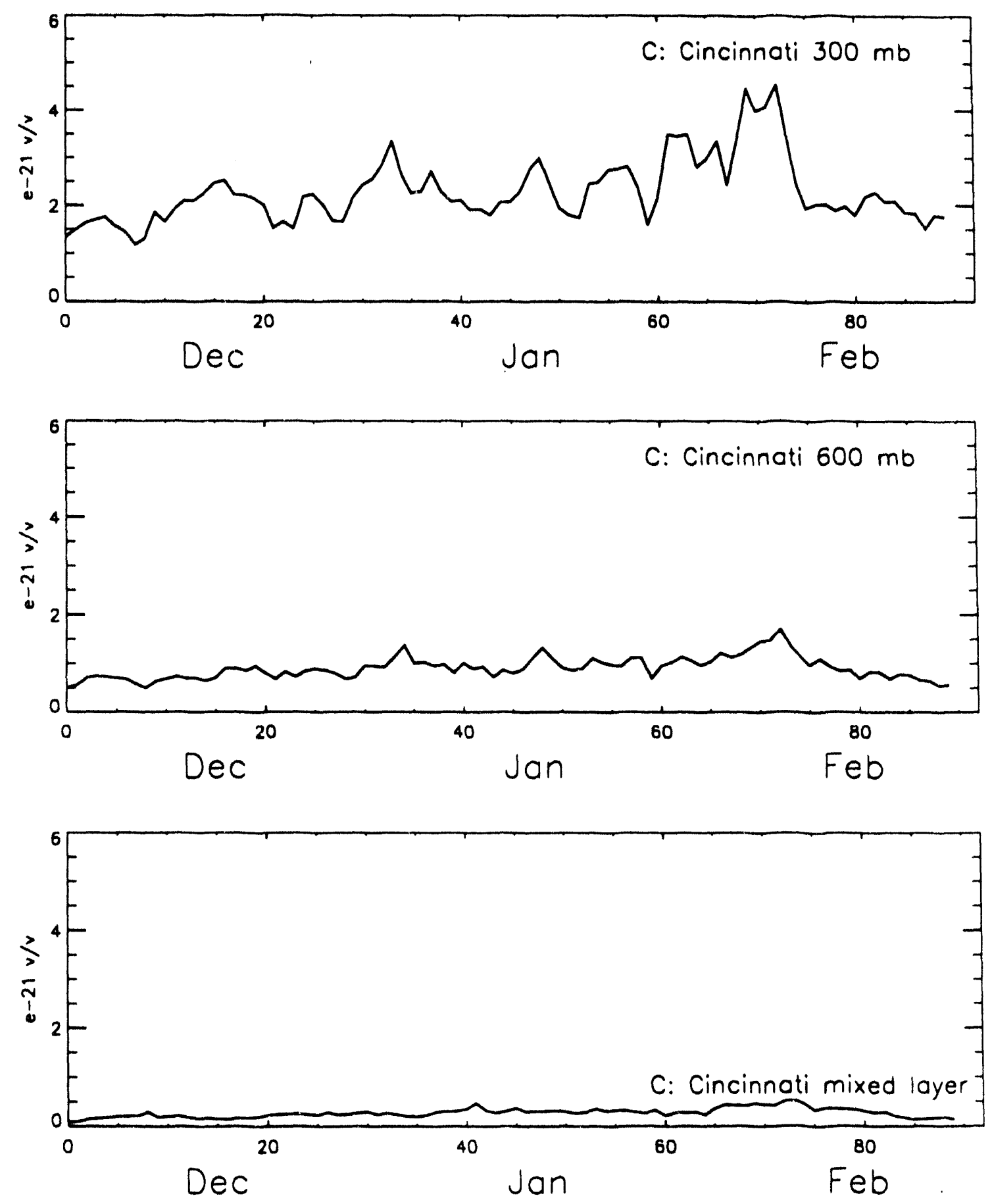
RADON-222 Mixing Ratio
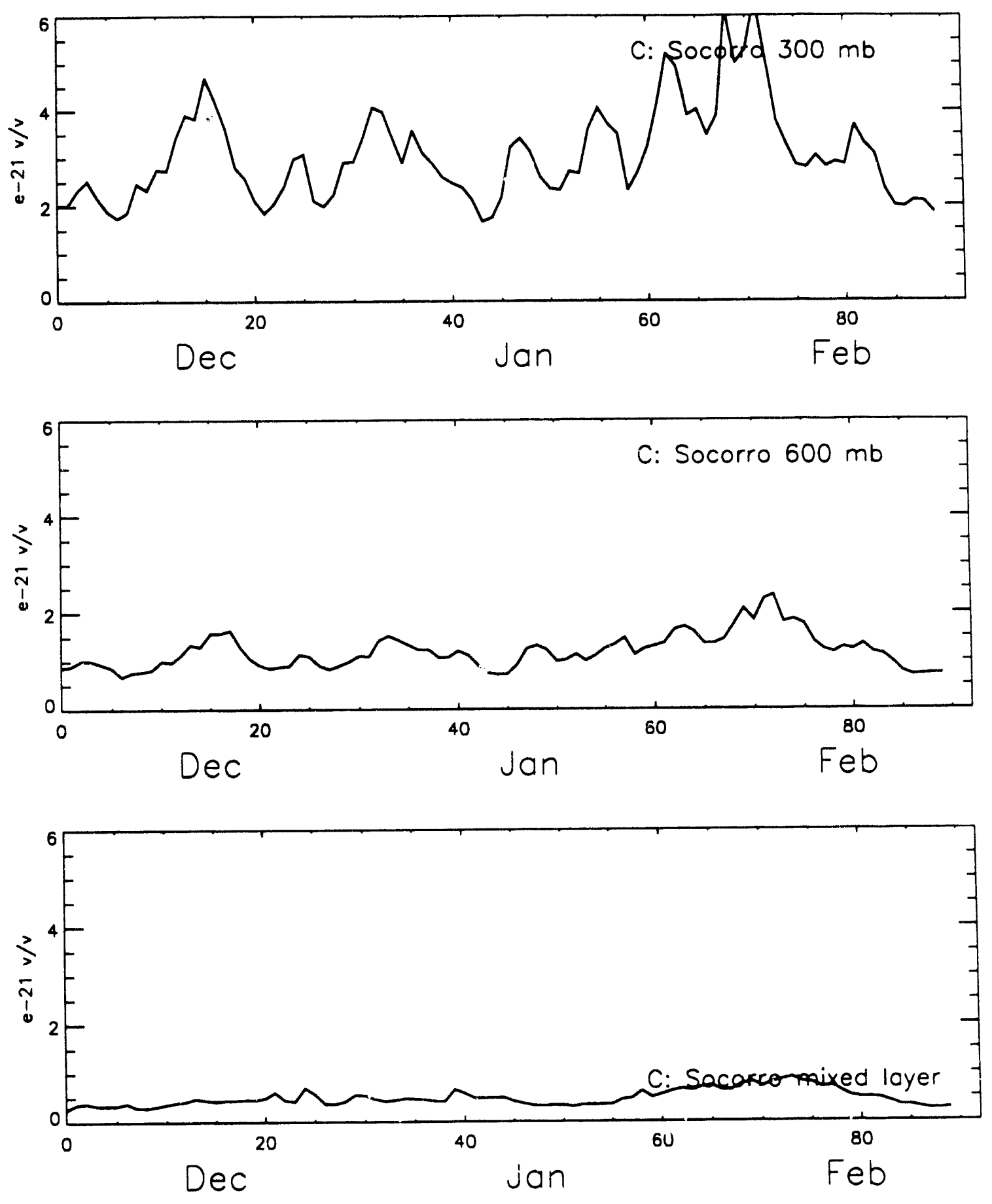
RADON-222 Mixing Ratio
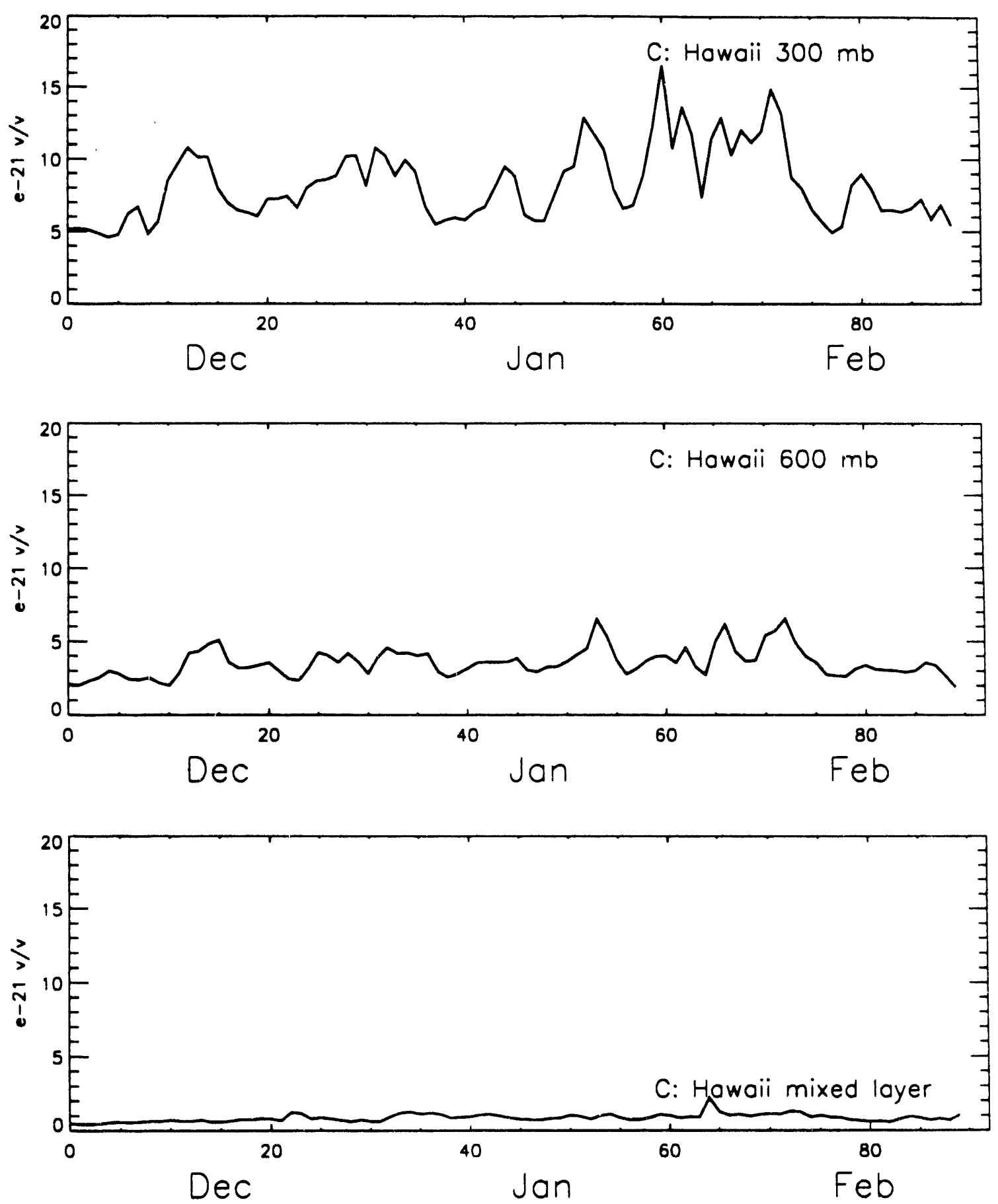
RADON-222 Mixing Ratio
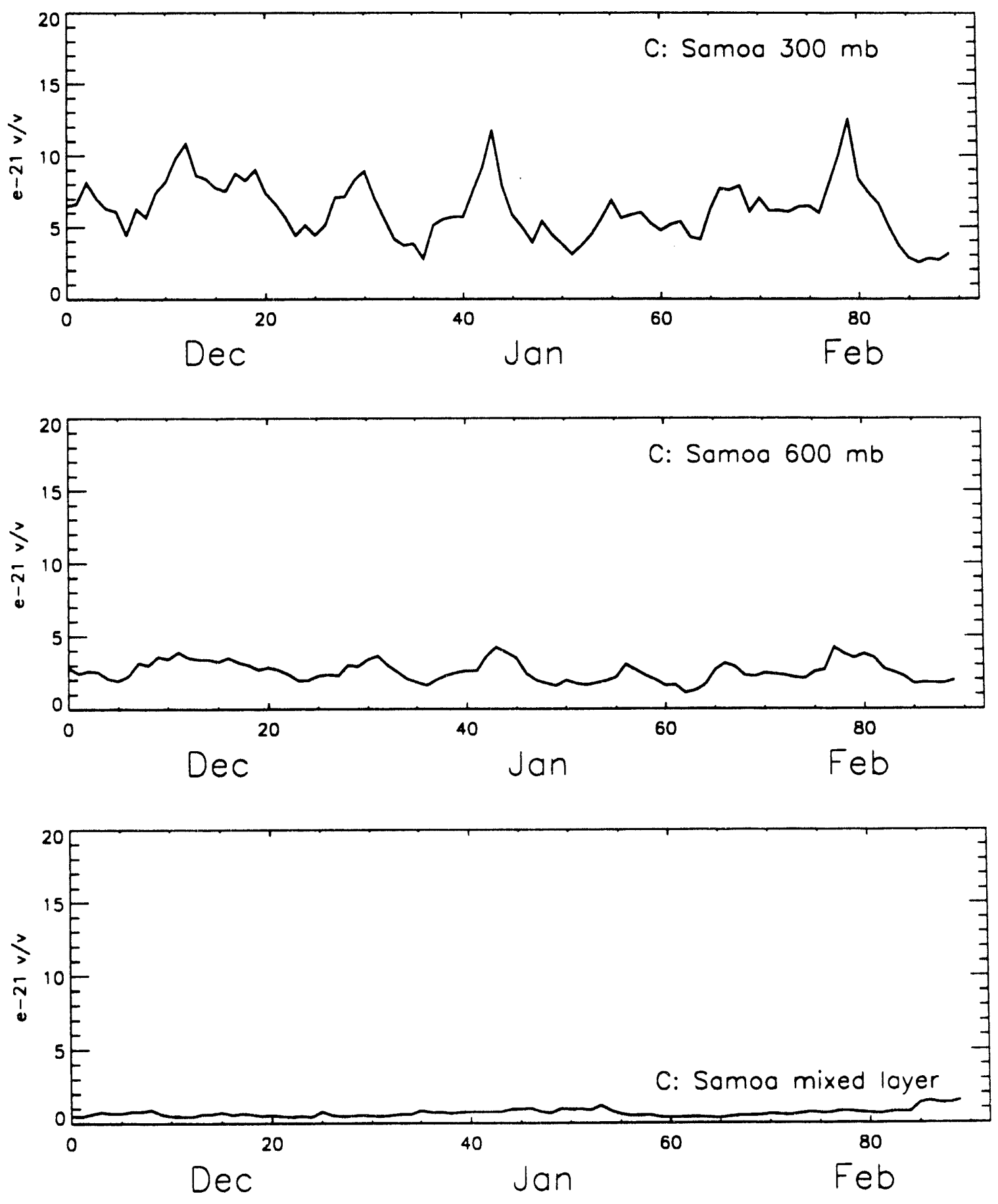
RADON-222 Mixing Ratio
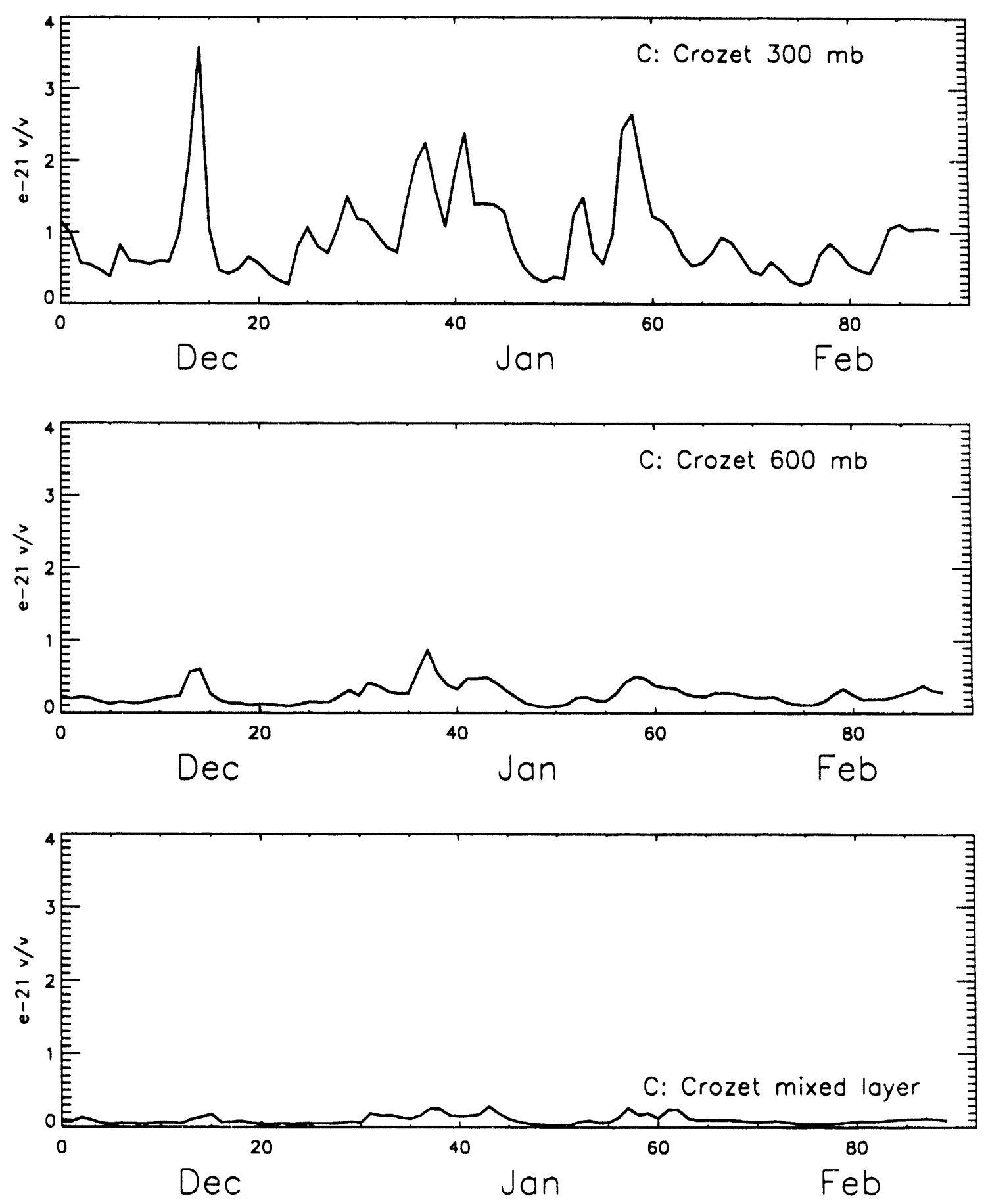
RADON-222 Mixing Ratio
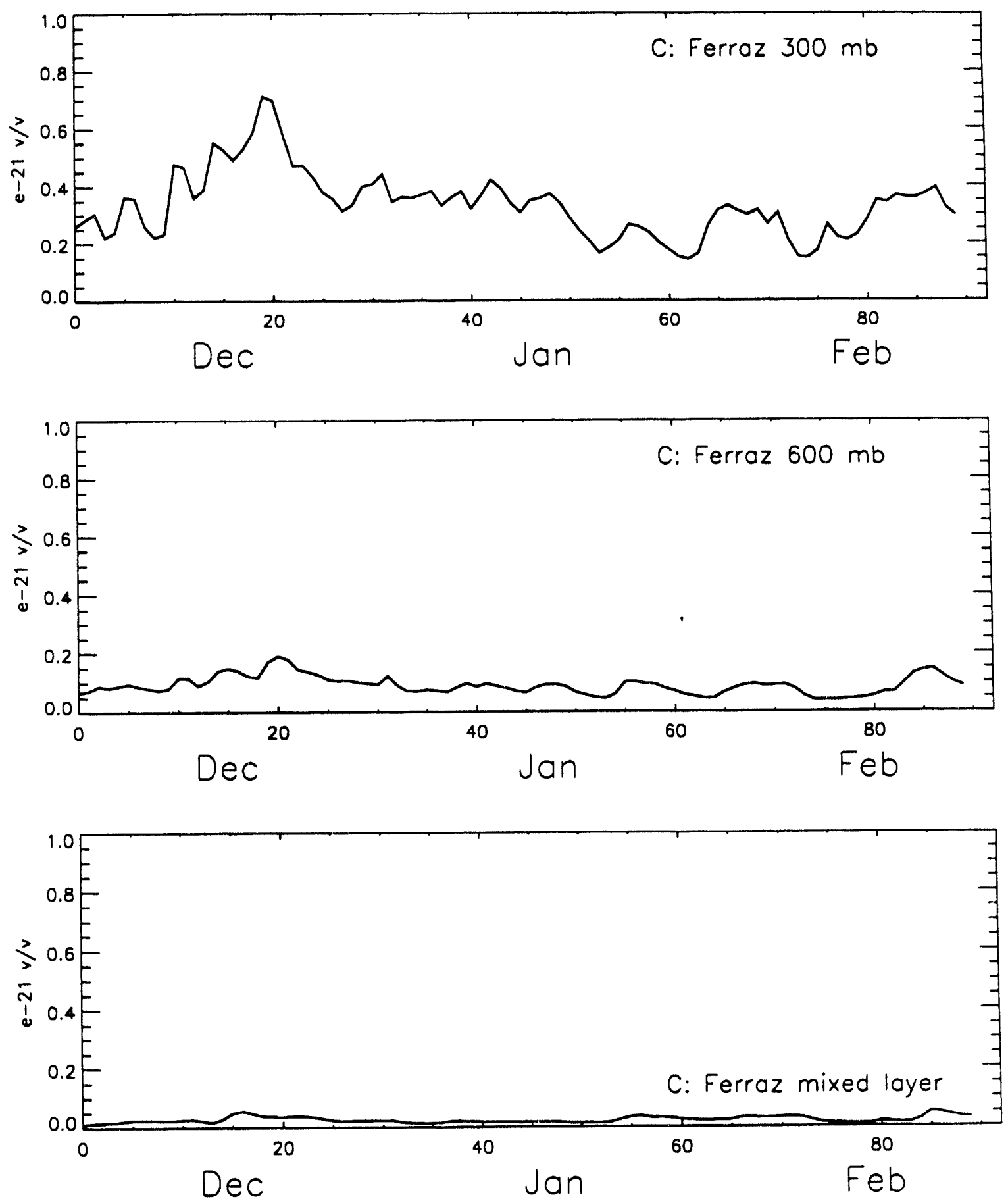


\section{EULERIAN GRANTOUR-LLNL Case C: Time series stadstics (E-21 v/v) Dec-Jan-Feb}

\begin{tabular}{|c|c|c|c|c|c|c|c|c|c|}
\hline site & level & mean & stdv & $\operatorname{var}^{\star} E-42$ & $\min$ & $1 / 4$ & median & $3 / 4$ & $\max$ \\
\hline \multirow{4}{*}{ Kirov } & $300 \mathrm{mb}$ & 0.56 & 0.28 & 0.08 & 0.23 & 0.39 & 0.47 & 0.70 & 1.39 \\
\hline & $600 \mathrm{mb}$ & 0.19 & 0.11 & 0.01 & 0.08 & 0.13 & 0.16 & 0.22 & 0.72 \\
\hline & mixed & 0.09 & 0.05 & 0.00 & 0.03 & 0.54 & 0.07 & 0.11 & 0.26 \\
\hline & surface & 0.08 & 0.04 & 0.00 & 0.03 & 0.54 & 0.07 & 0.11 & 0.23 \\
\hline \multirow{4}{*}{ Cincinnati } & $300 \mathrm{mb}$ & 2.29 & 0.68 & 0.47 & 1.19 & 1.80 & 2.15 & 2.52 & 4.53 \\
\hline & $600 \mathrm{mb}$ & 0.90 & 0.23 & 0.05 & 0.50 & 0.71 & 0.89 & 1.02 & 1.69 \\
\hline & mixed & 0.27 & 0.09 & 0.01 & 0.09 & 0.20 & 0.27 & 0.32 & 0.52 \\
\hline & surface & 0.26 & 0.09 & 0.01 & 0.08 & 0.19 & 0.26 & 0.31 & 0.50 \\
\hline \multirow{4}{*}{ Socorro } & $300 \mathrm{mb}$ & 3.05 & 0.99 & 0.98 & 1.66 & 2.32 & 2.87 & 3.61 & 6.35 \\
\hline & $600 \mathrm{mb}$ & 1.19 & 0.35 & 0.12 & 0.68 & 0.91 & 1.14 & 1.36 & 2.35 \\
\hline & mixed & 0.48 & 0.16 & 0.02 & 0.24 & 0.36 & 0.45 & 0.58 & 0.90 \\
\hline & surface & 0.46 & 0.15 & 0.02 & 0.23 & 0.34 & 0.43 & 0.56 & 0.86 \\
\hline \multirow{4}{*}{ Hawaii } & $300 \mathrm{mb}$ & 8.22 & 2.56 & 6.53 & 4.60 & 6.30 & 7.96 & 10.12 & 16.54 \\
\hline & $600 \mathrm{mb}$ & 3.55 & 0.97 & 0.95 & 1.92 & 2.84 & 3.55 & 4.04 & 6.55 \\
\hline & mixed & 0.86 & 0.26 & 0.07 & 0.43 & 0.67 & 0.84 & 1.03 & 2.20 \\
\hline & surface & 0.80 & 0.24 & 0.06 & 0.41 & 0.62 & 0.78 & 0.92 & 2.11 \\
\hline \multirow{4}{*}{ Samoa } & $300 \mathrm{mb}$ & 6.24 & 2.03 & 4.12 & 2.51 & 4.98 & 6.11 & 7.50 & 12.54 \\
\hline & $600 \mathrm{mb}$ & 2.56 & 0.71 & 0.51 & 1.11 & 1.97 & 2.48 & 3.05 & 4.23 \\
\hline & mixed & 0.68 & 0.24 & 0.06 & 0.34 & 0.49 & 0.64 & 0.77 & 1.52 \\
\hline & surface & 0.62 & 0.21 & 0.04 & 0.33 & 0.47 & 0.59 & 0.73 & 1.32 \\
\hline \multirow{4}{*}{ Crozet } & $300 \mathrm{mb}$ & 0.94 & 0.59 & 0.35 & 0.27 & 0.54 & 0.80 & 1.15 & 3.58 \\
\hline & $600 \mathrm{mb}$ & 0.26 & 0.14 & 0.02 & 0.08 & 0.15 & 0.23 & 0.31 & 0.86 \\
\hline & mixed & 0.10 & 0.06 & 0.00 & 0.03 & 0.05 & 0.08 & 0.12 & 0.29 \\
\hline & surface & 0.10 & 0.06 & 0.00 & 0.02 & 0.05 & 0.08 & 0.12 & 0.27 \\
\hline \multirow{4}{*}{ Ferraz } & $300 \mathrm{mb}$ & 0.33 & 0.11 & 0.01 & 0.14 & 0.26 & 0.34 & 0.38 & 0.71 \\
\hline & $600 \mathrm{mb}$ & $8.9 e-2$ & $3.2 \mathrm{e}-2$ & $1.0 e-3$ & $3.9 e-2$ & $6.8 e-2$ & $8.8 e-2$ & $1.0 e-1$ & $1.9 e-1$ \\
\hline & mixed & $2.4 e-2$ & $1.0 \mathrm{e}-2$ & $1.0 e-4$ & $8.7 e-3$ & $1.6 e-2$ & $2.2 \mathrm{e}-2$ & $3.0 e-2$ & $5.6 e-2$ \\
\hline & surface & $2.3 e-2$ & $9.8 \mathrm{e}-3$ & $9.7 e-5$ & $8.4 e-3$ & $1.5 \mathrm{e} \cdot 2$ & $2.0 e-2$ & $2.9 e-2$ & $5.5 e-2$ \\
\hline
\end{tabular}



EULERIAN GRANTOUR-LLNL
DJF
C: Mixed Layer

RADON-222 Mixing Ratio [*e21 v/v]

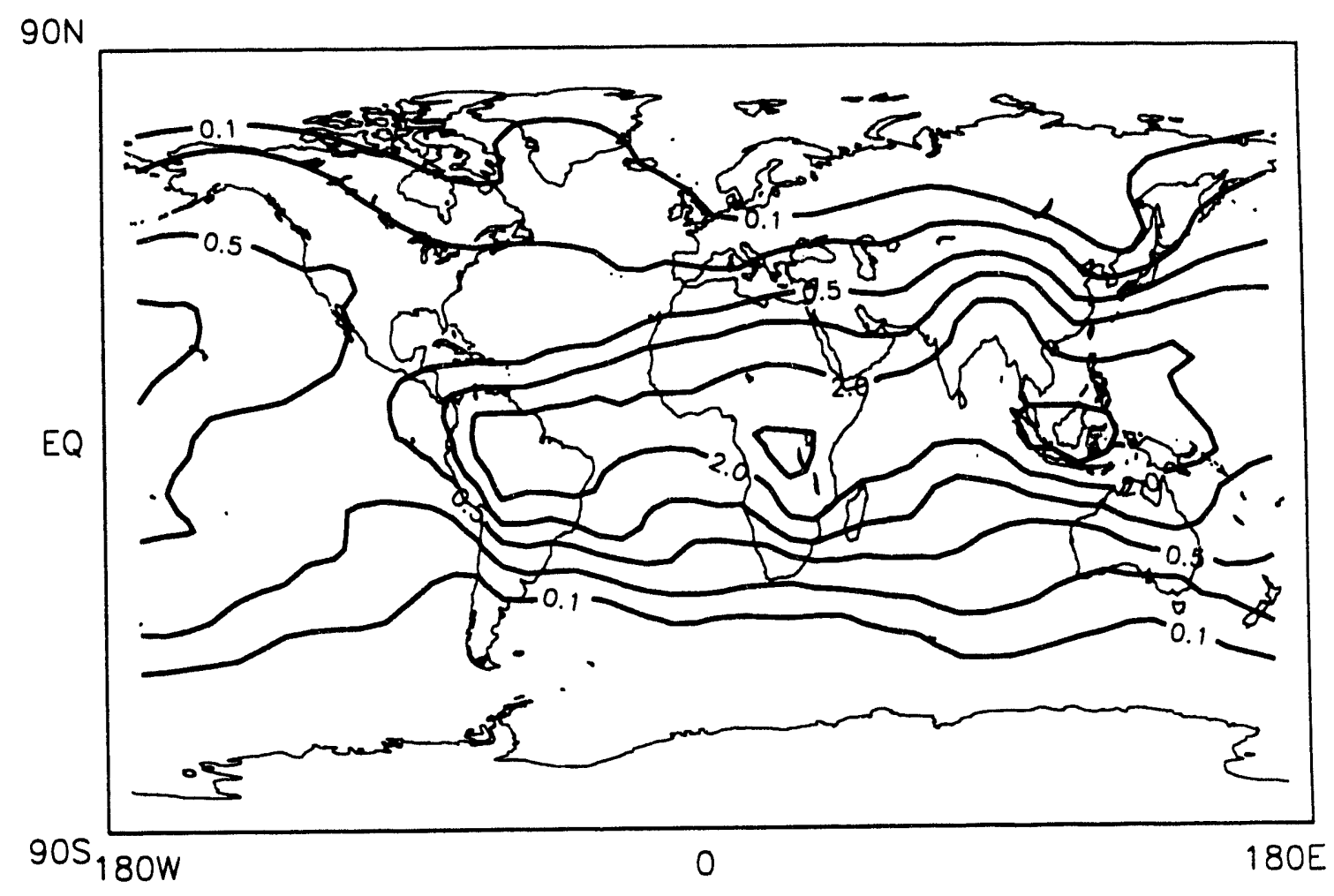




\section{EULERIAN GRANTOUR-LLNL

\section{C: $600 \mathrm{mb}$}

\section{RADON-222 Mixing Rotio [*e21 v/v]}

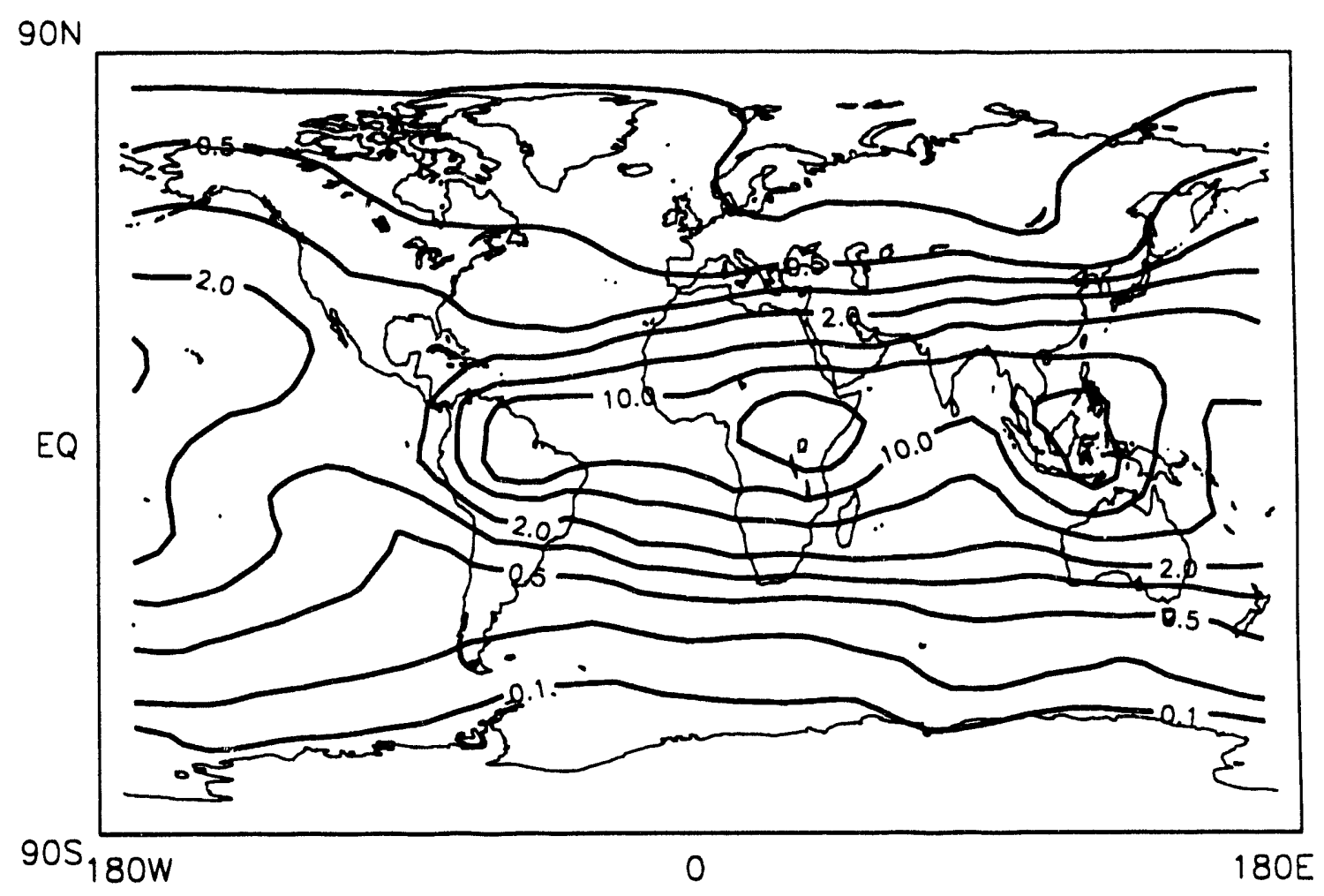


EULERIAN GRANTOUR-LLNL

DJF

\section{C: $300 \mathrm{mb}$}

RADON-222 Mixing Rotio $[* e 21 \mathrm{v} / \mathrm{v}]$

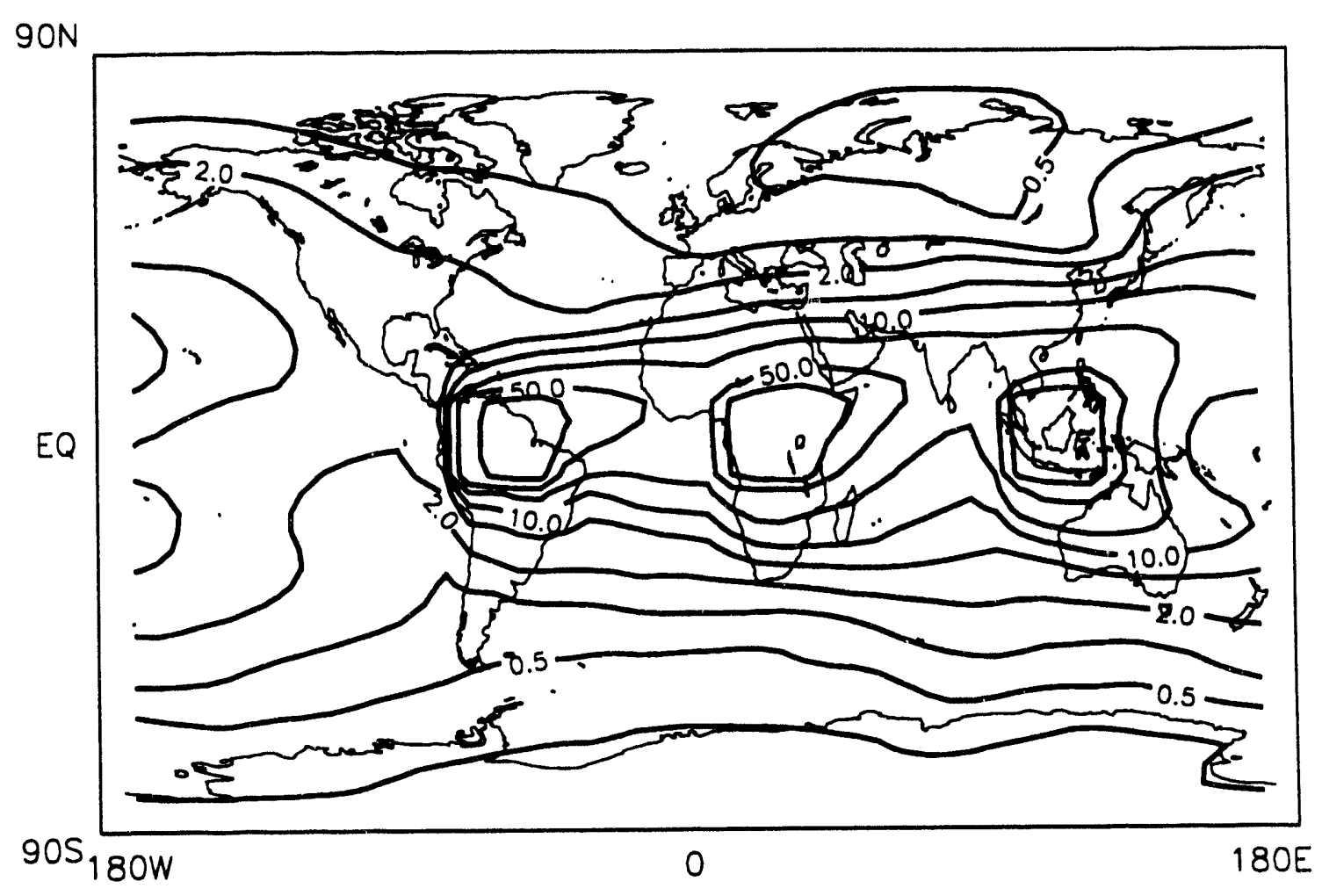


EULERIAN GRANTOUR-LLNL

DJF

C: Zonal Avg

RADON-222 Mixing Ratio [*e21 v/v]

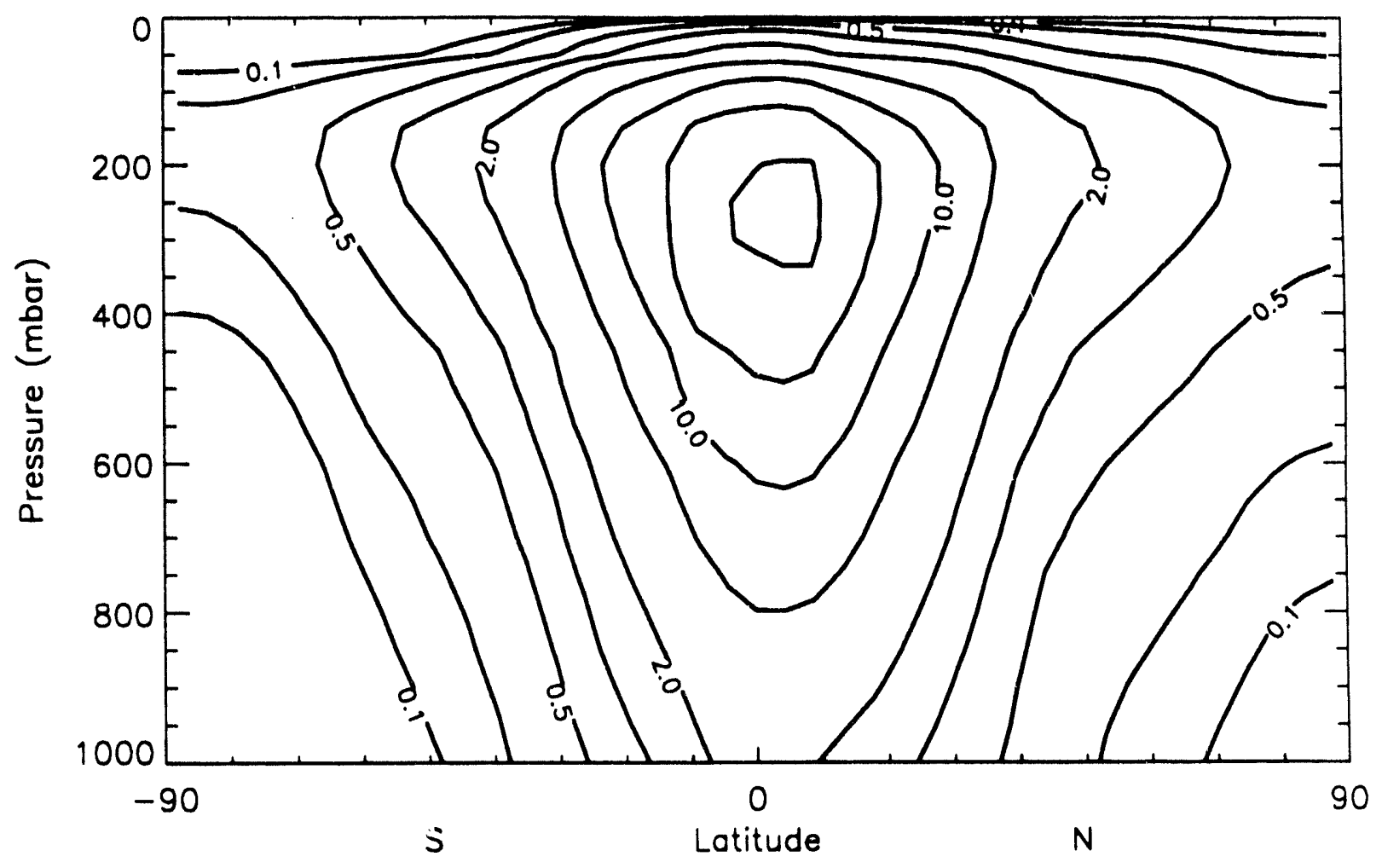


EULERIAN GRANTOUR-LLNL

\section{C: OEW}

$$
\text { RADON-222 Mixing Rotio [*e21 v/v] }
$$

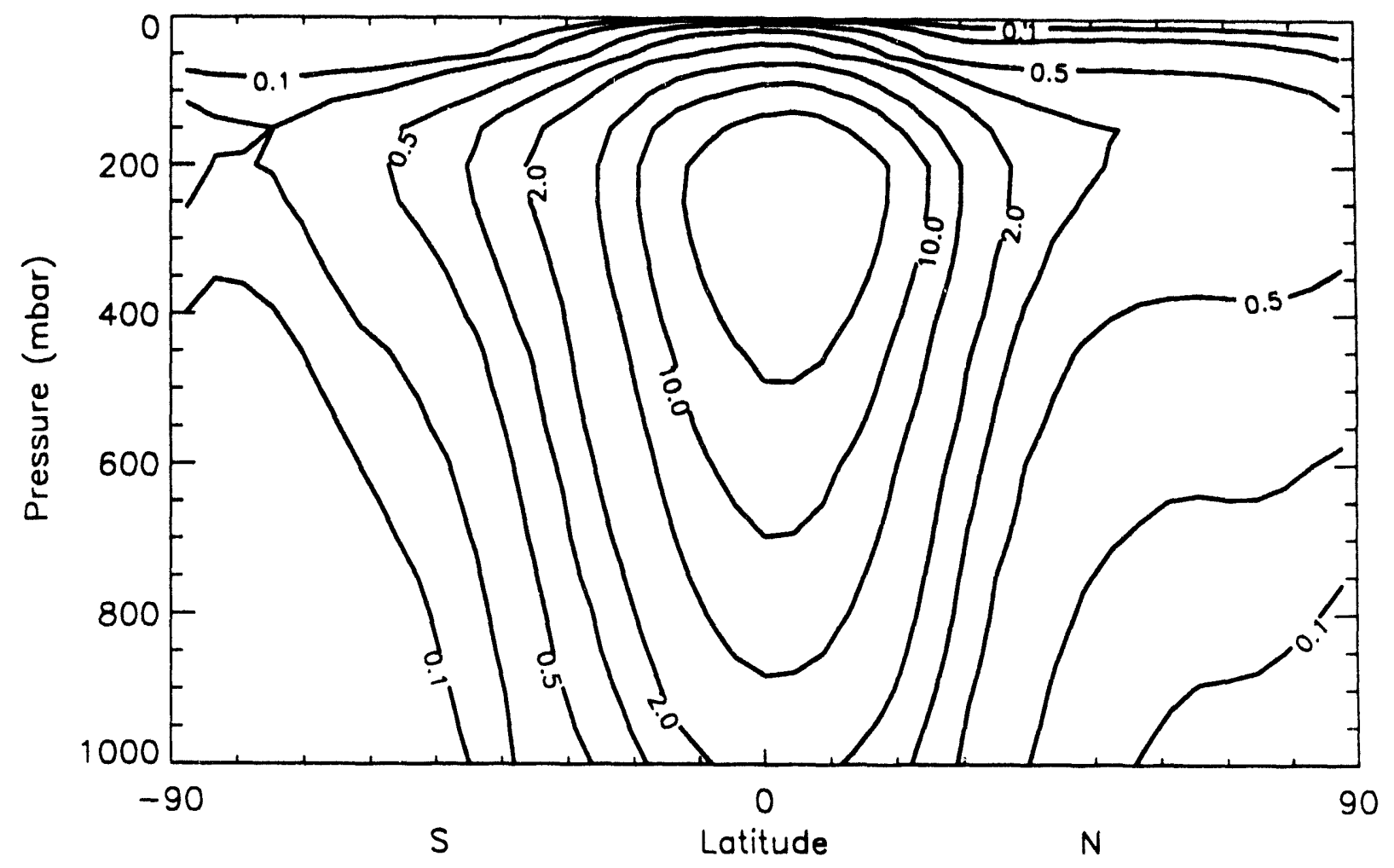




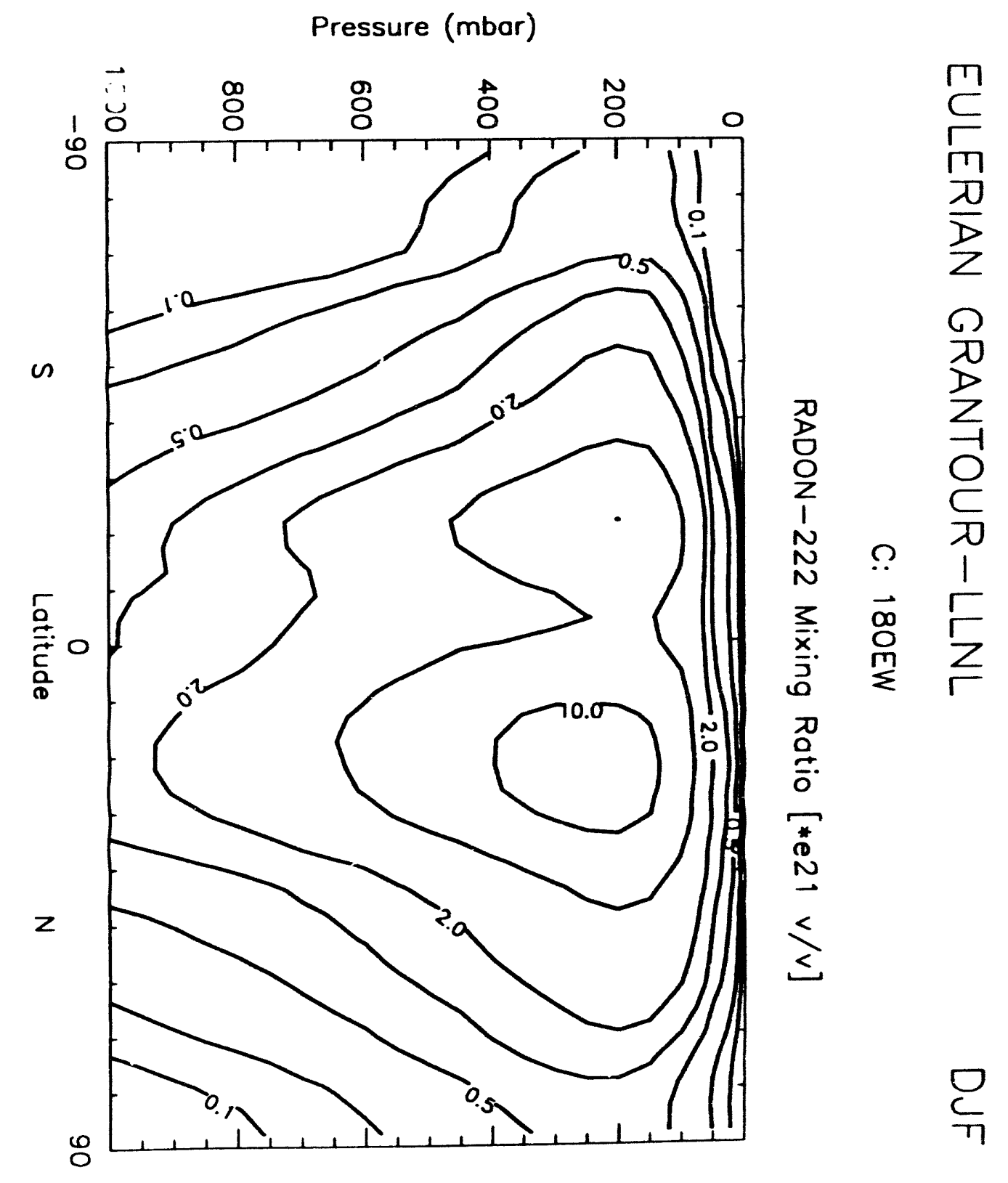


RADON-222 Mixing Ratio
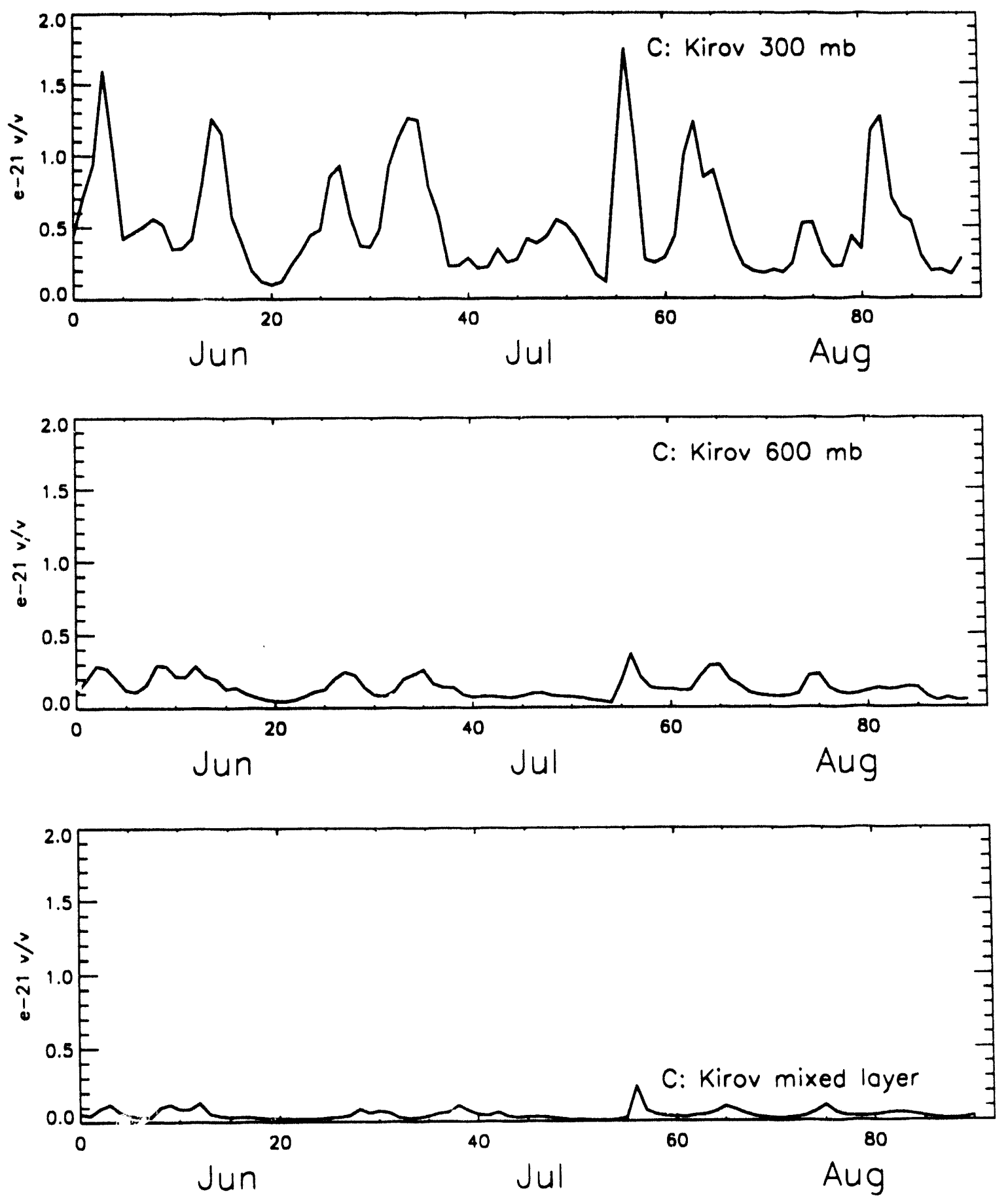
RADON-222 Mixing Ratio
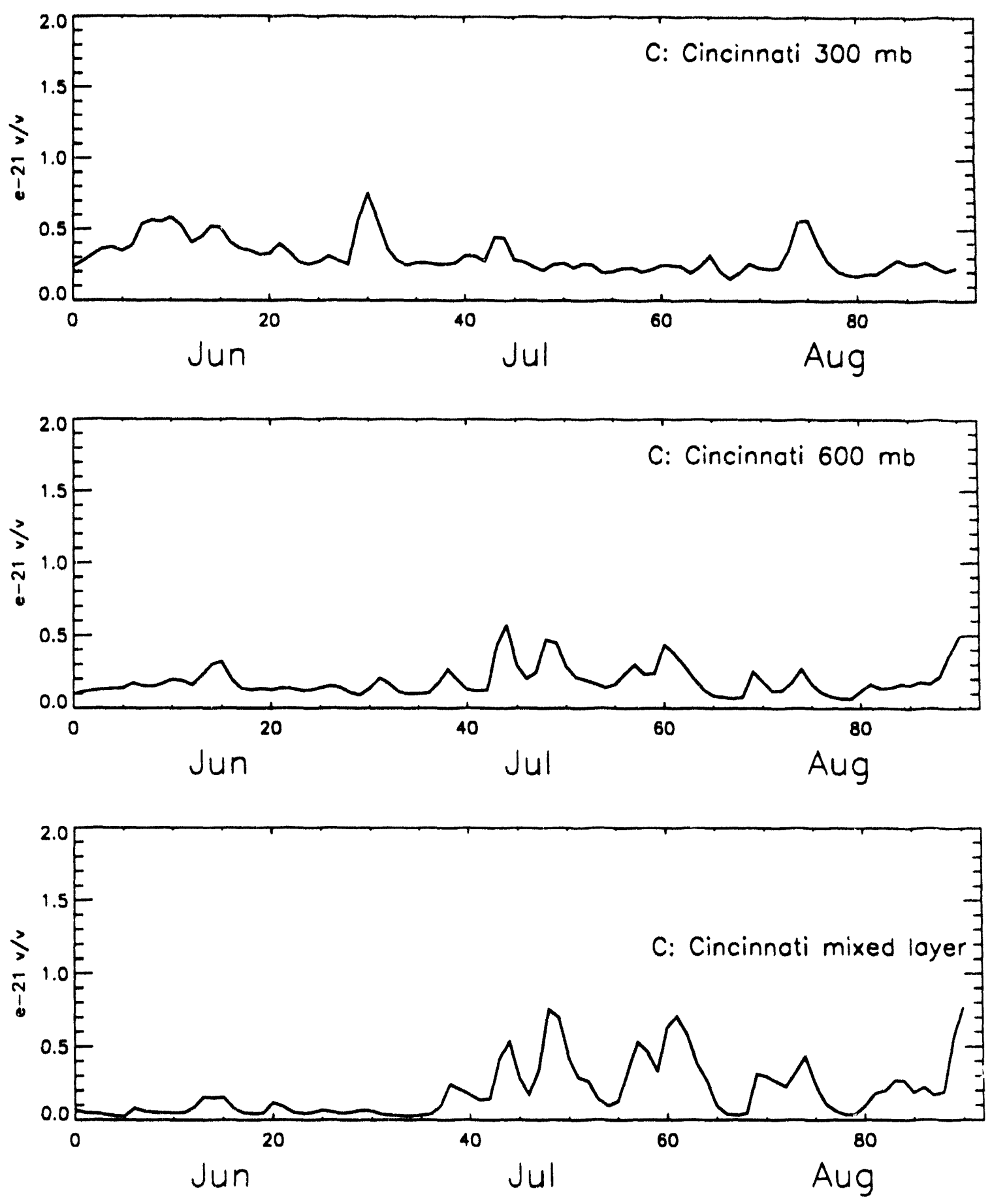

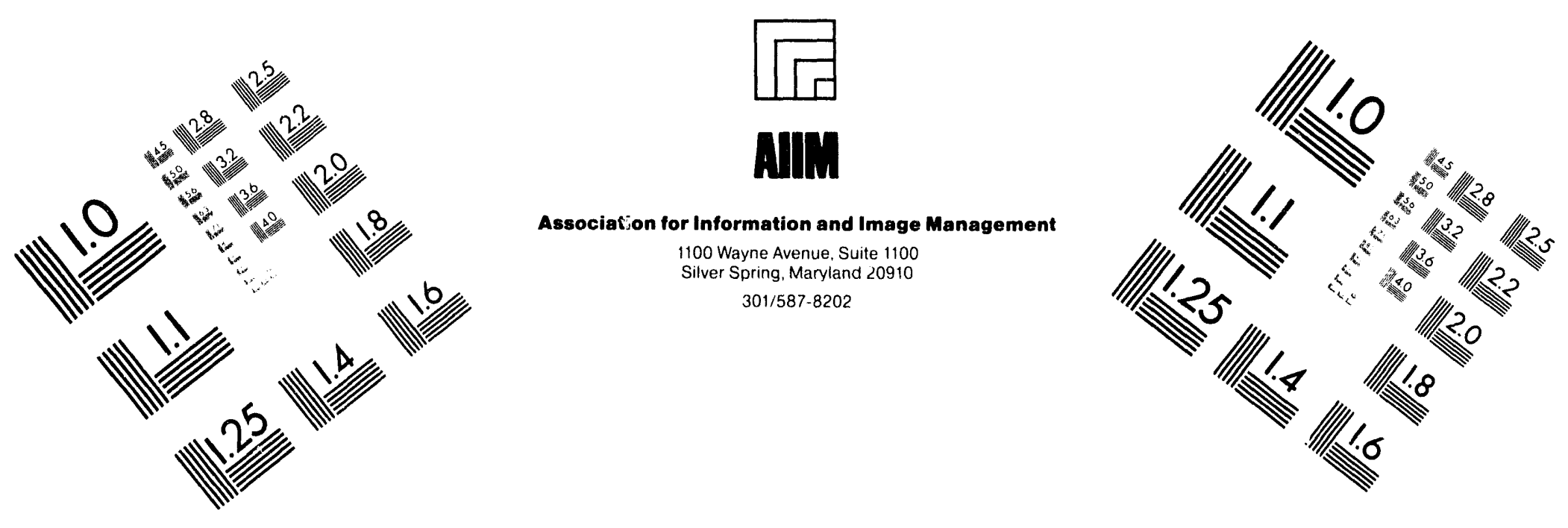

Centimeter

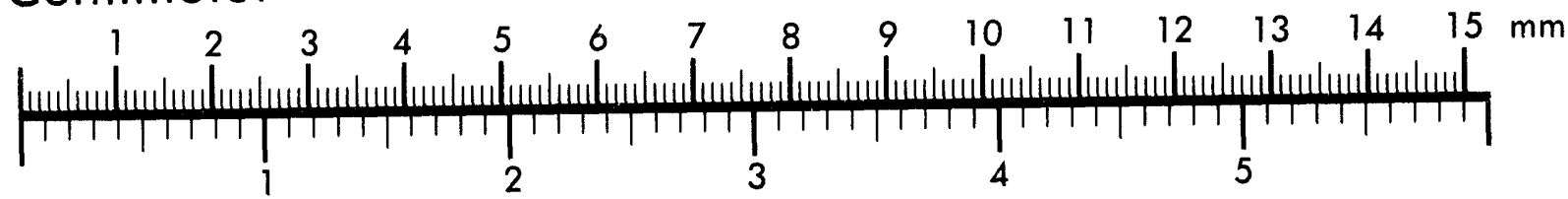
Inches
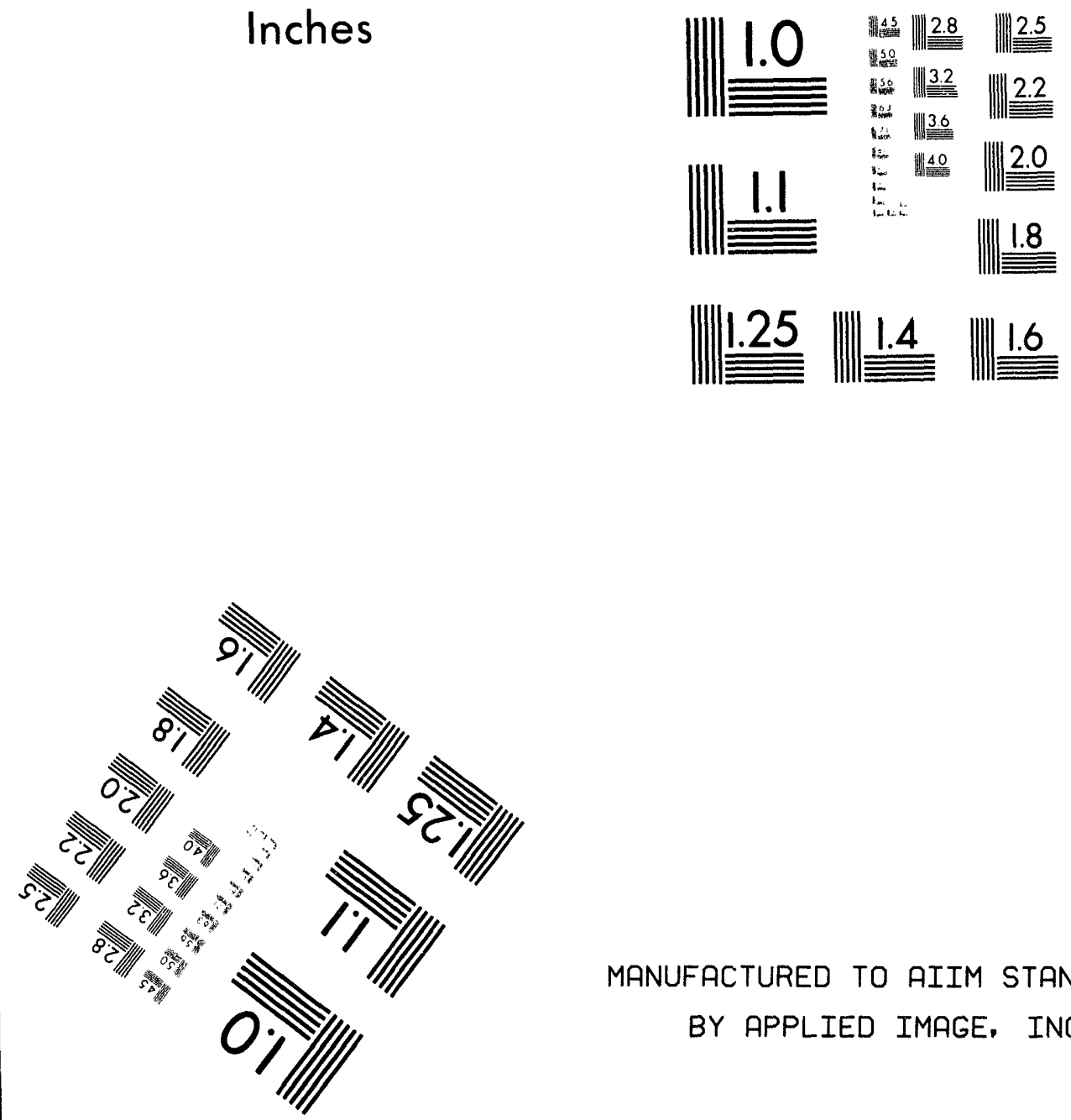

MANUFACTURED TO AIIM STANDARDS

BY APPLIED IMAGE, INC.

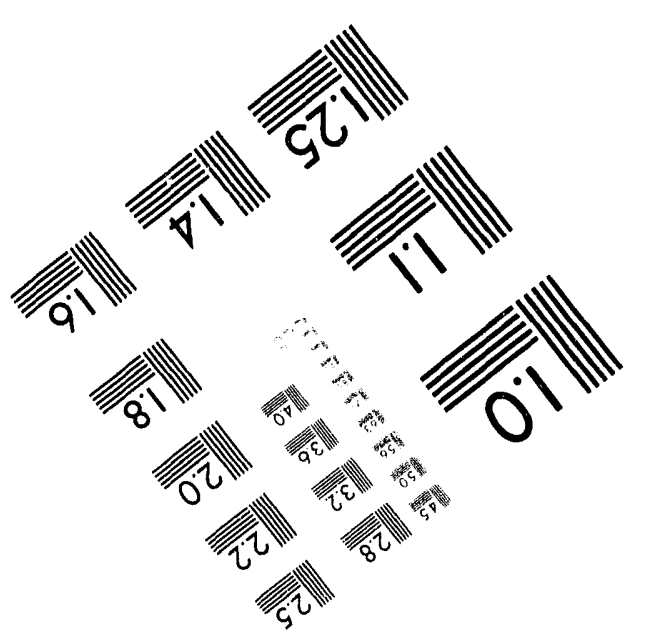



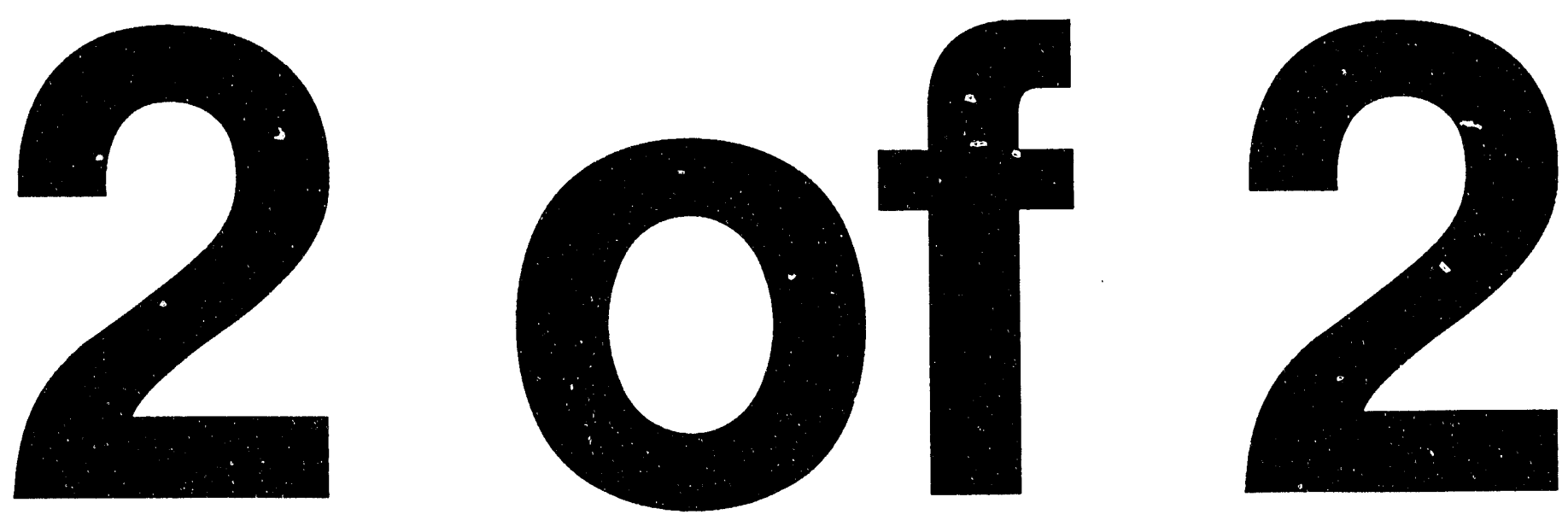
RADON-222 Mixing Ratio
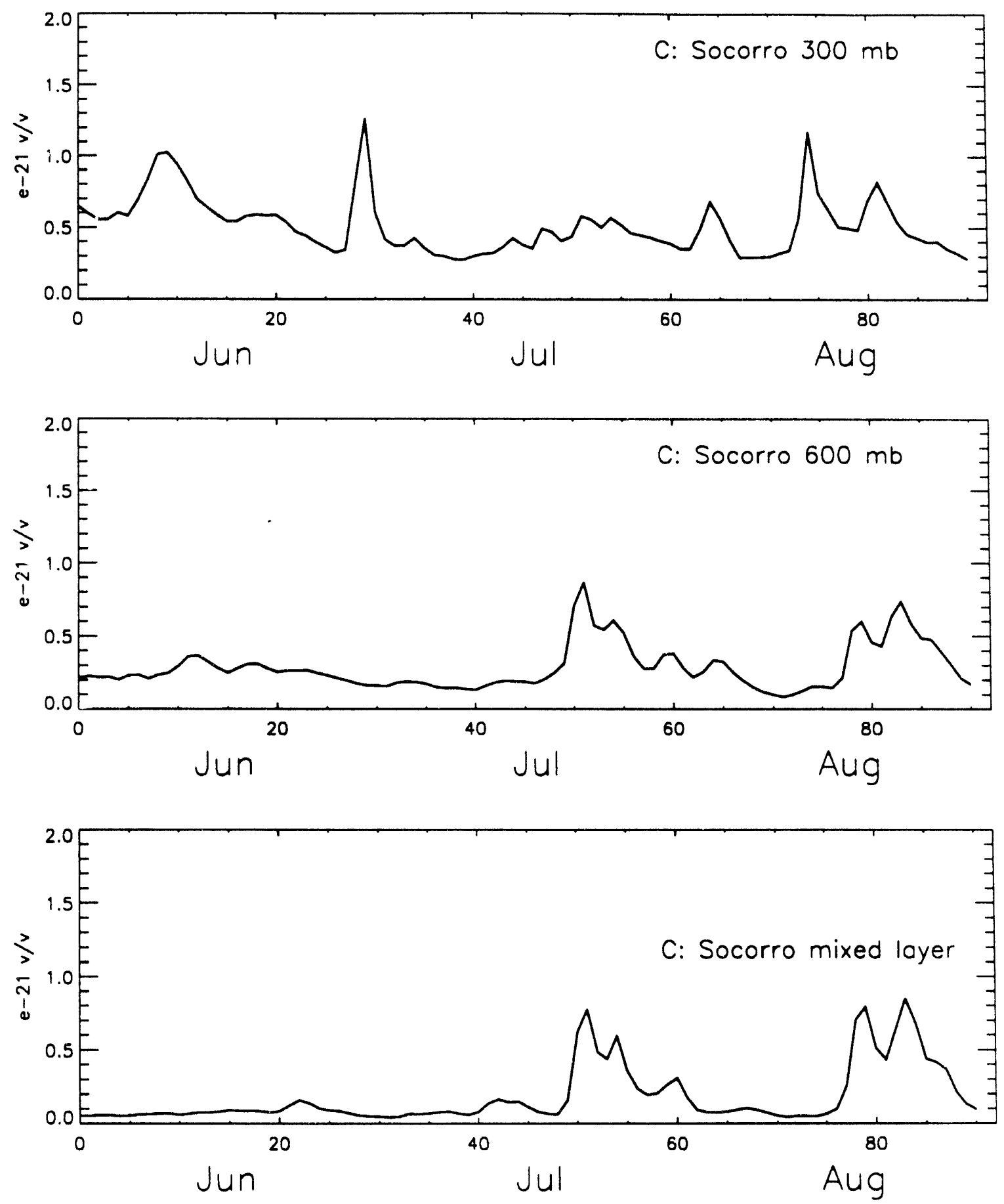
RADON-222 Mixing Ratio
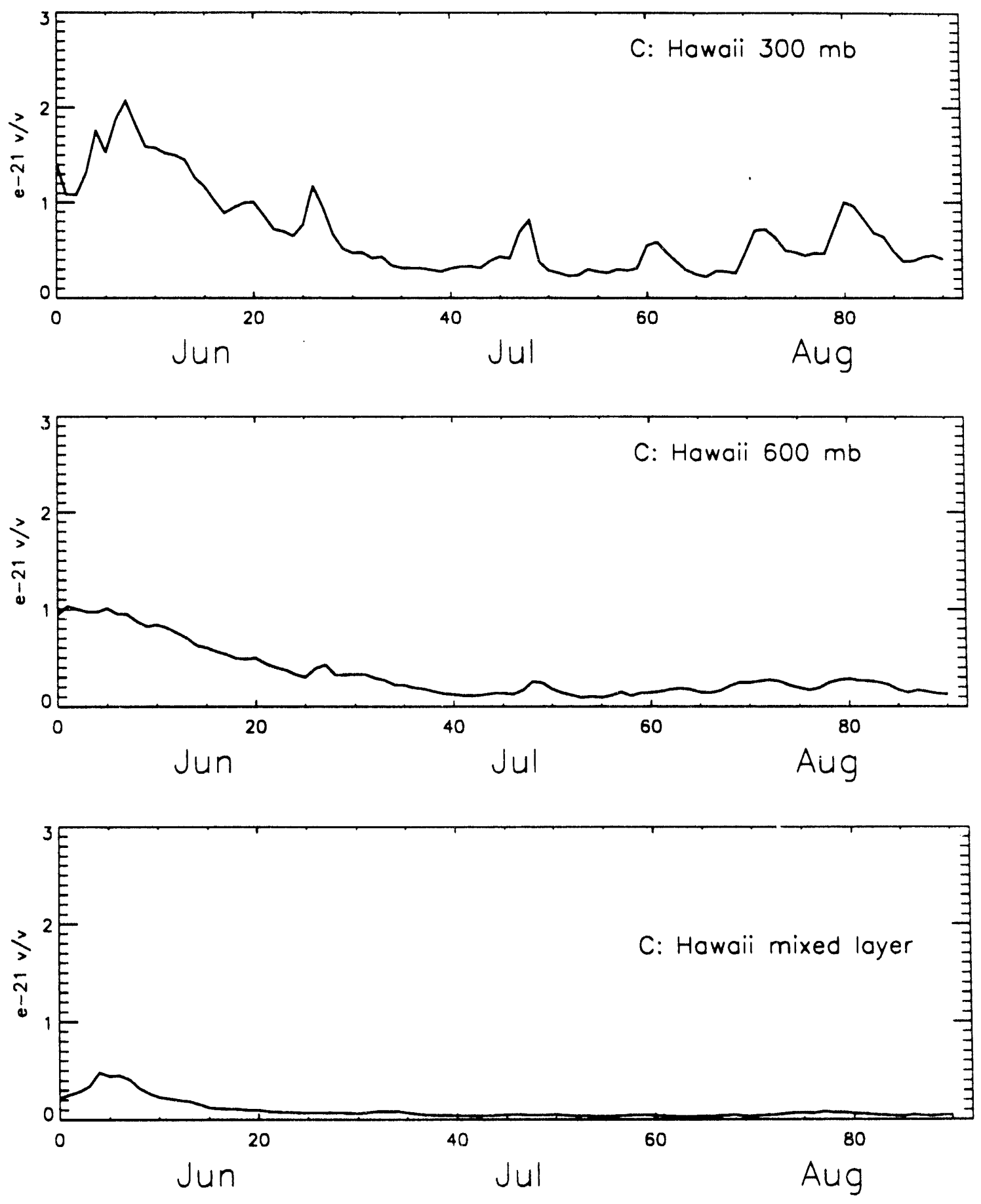
EULERIAN GRANTOUR-LLNL

Jun-Jul-Aug

RADON-222 Mixing Ratio
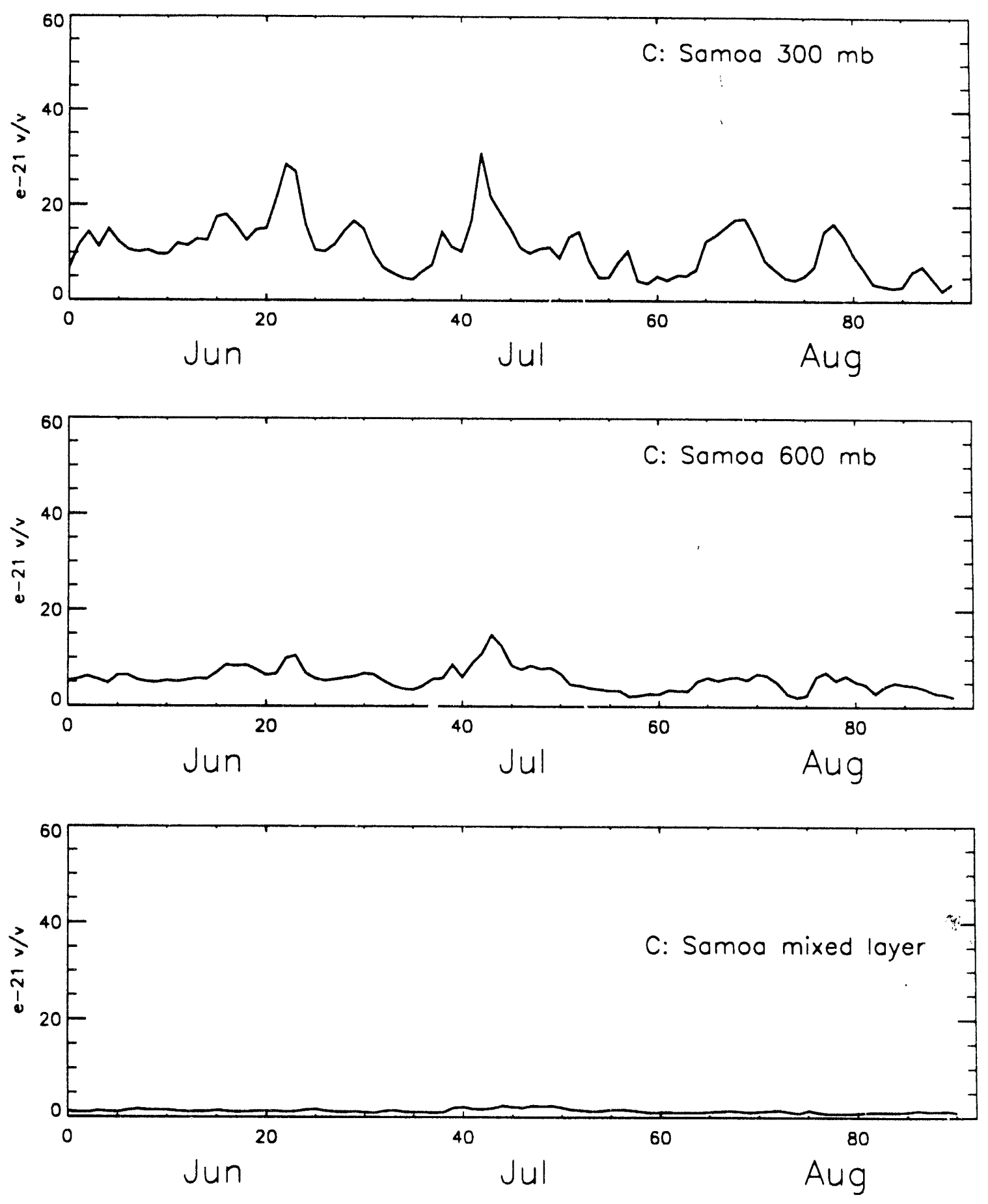
EULERIAN GRANTOUR-LLNL

Jun-Jul-Aug

RADON-222 Mixing Ratio
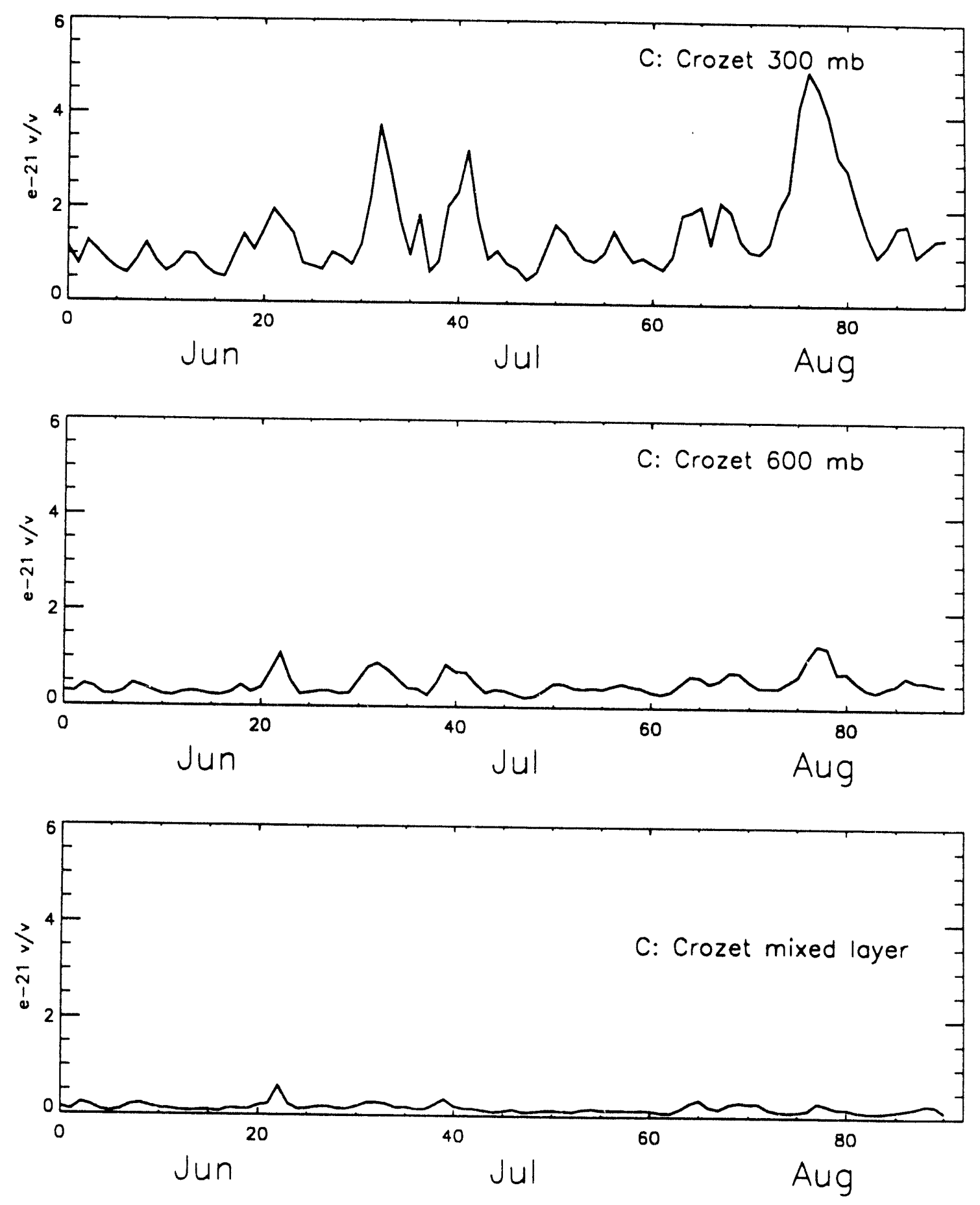
RADON-222 Mixing Ratio
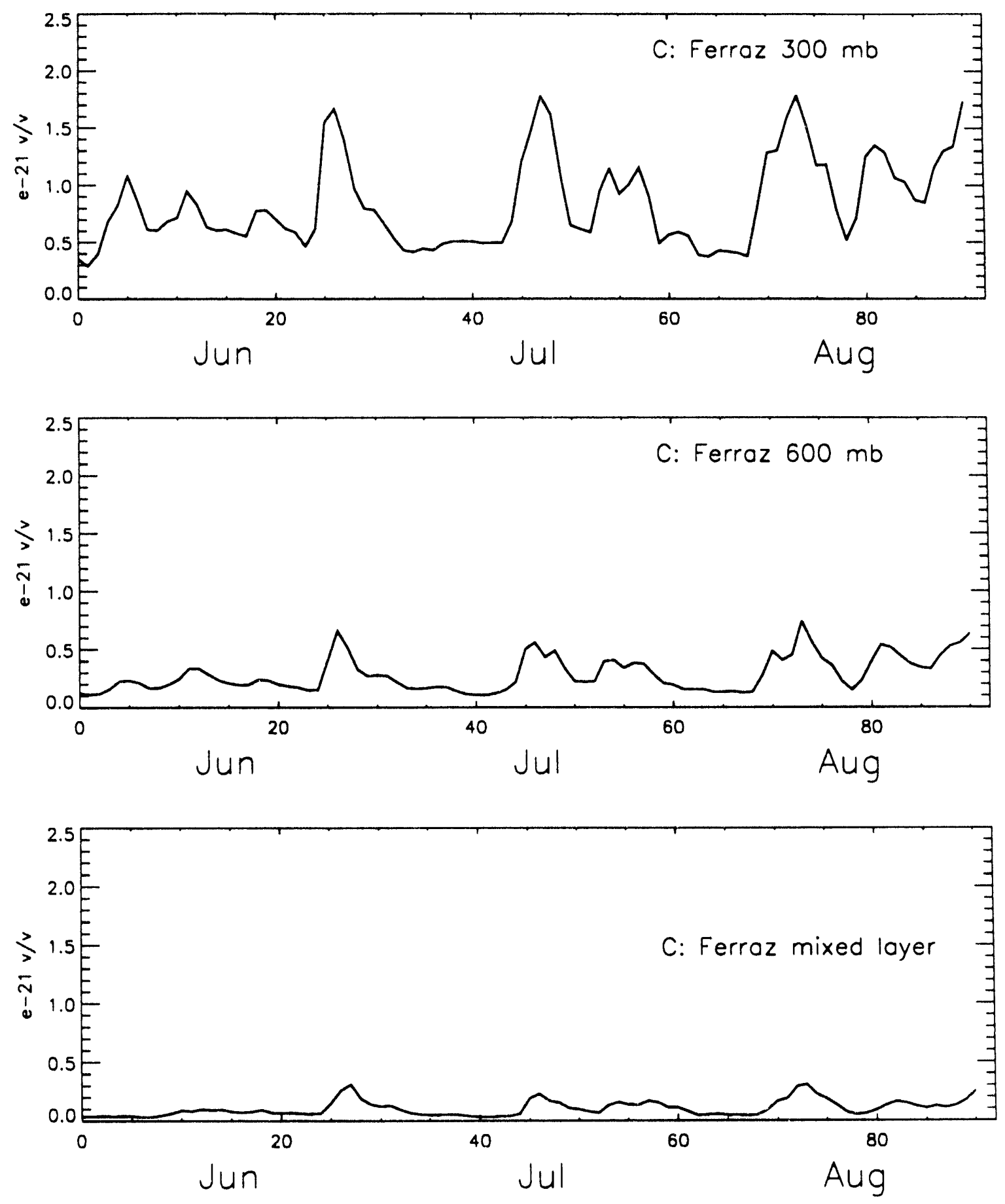


\begin{tabular}{|c|c|c|c|c|c|c|c|c|c|}
\hline \multicolumn{10}{|c|}{ EULERIAN GRANTOUR-LLNL Case C: Time series statistics (E-21 v/v) Jun-Jul-Aug } \\
\hline site & level & mean & stdv & $\operatorname{var}^{\star} \mathrm{E}-42$ & $\min$ & $1 / 4$ & median & $3 / 4$ & $\max$ \\
\hline Kirov & $\begin{array}{c}300 \mathrm{mb} \\
600 \mathrm{mb} \\
\text { mixed } \\
\text { surface }\end{array}$ & $\begin{array}{l}0.53 \\
0.14 \\
0.05 \\
0.04 \\
\end{array}$ & $\begin{array}{l}0.36 \\
0.07 \\
0.03 \\
0.03 \\
\end{array}$ & $\begin{array}{r}0.13 \\
0.01 \\
0.00 \\
0.00 \\
\end{array}$ & $\begin{array}{l}0.10 \\
0.03 \\
0.01 \\
0.01 \\
\end{array}$ & $\begin{array}{l}0.25 \\
0.08 \\
0.02 \\
0.02 \\
\end{array}$ & $\begin{array}{l}0.42 \\
0.12 \\
0.03 \\
0.03 \\
\end{array}$ & $\begin{array}{l}0.71 \\
0.20 \\
0.06 \\
0.05 \\
\end{array}$ & $\begin{array}{l}1.75 \\
0.36 \\
0.24 \\
0.13 \\
\end{array}$ \\
\hline Cincinnati & $\begin{array}{c}300 \mathrm{mb} \\
600 \mathrm{mb} \\
\text { mixed } \\
\text { surface }\end{array}$ & \begin{tabular}{l|}
0.31 \\
0.19 \\
0.19 \\
0.19 \\
\end{tabular} & $\begin{array}{l}0.12 \\
0.10 \\
0.19 \\
0.19 \\
\end{array}$ & \begin{tabular}{l|}
0.01 \\
0.01 \\
0.04 \\
0.03 \\
\end{tabular} & $\begin{array}{l}0.15 \\
0.07 \\
0.03 \\
0.03 \\
\end{array}$ & \begin{tabular}{l|}
0.23 \\
0.13 \\
0.05 \\
0.05 \\
\end{tabular} & $\begin{array}{l}0.26 \\
0.16 \\
0.13 \\
0.12 \\
\end{array}$ & $\begin{array}{l}0.36 \\
0.21 \\
0.27 \\
0.27 \\
\end{array}$ & \begin{tabular}{l|}
0.76 \\
0.57 \\
0.77 \\
0.76 \\
\end{tabular} \\
\hline Socorro & $\begin{array}{c}300 \mathrm{mb} \\
600 \mathrm{mb} \\
\text { mixed } \\
\text { surface }\end{array}$ & \begin{tabular}{l|}
0.51 \\
0.28 \\
0.18 \\
0.17 \\
\end{tabular} & \begin{tabular}{l|}
0.20 \\
0.16 \\
0.20 \\
0.19 \\
\end{tabular} & $\begin{array}{l}0.04 \\
0.02 \\
0.04 \\
0.04 \\
\end{array}$ & $\begin{array}{l}0.27 \\
0.08 \\
0.04 \\
0.04 \\
\end{array}$ & $\begin{array}{l}0.36 \\
0.18 \\
0.07 \\
0.06 \\
\end{array}$ & $\begin{array}{l}0.47 \\
0.24 \\
0.08 \\
0.08 \\
\end{array}$ & $\begin{array}{l}0.59 \\
0.33 \\
0.19 \\
0.19 \\
\end{array}$ & $\begin{array}{l}1.27 \\
0.87 \\
0.85 \\
0.80 \\
\end{array}$ \\
\hline Hawaii & $\begin{array}{c}300 \mathrm{mb} \\
600 \mathrm{mb} \\
\text { mixed } \\
\text { surface }\end{array}$ & $\begin{array}{l}0.69 \\
0.34 \\
0.10 \\
0.09 \\
\end{array}$ & $\begin{array}{l}0.45 \\
0.27 \\
0.10 \\
0.10 \\
\end{array}$ & $\begin{array}{l}0.20 \\
0.07 \\
0.01 \\
0.01 \\
\end{array}$ & $\begin{array}{l}0.22 \\
0.09 \\
0.03 \\
0.03 \\
\end{array}$ & $\begin{array}{l}0.31 \\
0.15 \\
0.04 \\
0.04 \\
\end{array}$ & $\begin{array}{l}0.48 \\
0.25 \\
0.05 \\
0.05 \\
\end{array}$ & $\begin{array}{l}0.95 \\
0.43 \\
0.08 \\
0.08 \\
\end{array}$ & $\begin{array}{l}2.07 \\
1.03 \\
0.48 \\
0.44 \\
\end{array}$ \\
\hline Samoa & $\begin{array}{c}300 \mathrm{mb} \\
600 \mathrm{mb} \\
\text { mixed } \\
\text { surface }\end{array}$ & $\begin{array}{r}11.00 \\
5.70 \\
1.31 \\
1.23 \\
\end{array}$ & $\begin{array}{l}5.69 \\
2.30 \\
0.36 \\
0.33 \\
\end{array}$ & $\begin{array}{r}32.39 \\
5.31 \\
0.13 \\
0.11 \\
\end{array}$ & \begin{tabular}{l|}
2.21 \\
1.98 \\
0.77 \\
0.74 \\
\end{tabular} & $\begin{array}{l}6.26 \\
4.28 \\
1.07 \\
1.01 \\
\end{array}$ & $\begin{array}{r}10.65 \\
5.56 \\
1.23 \\
1.12 \\
\end{array}$ & $\begin{array}{r}14.62 \\
6.78 \\
1.44 \\
1.33 \\
\end{array}$ & $\begin{array}{r}30.90 \\
14.86 \\
2.36 \\
2.17 \\
\end{array}$ \\
\hline Crozet & $\begin{array}{c}300 \mathrm{mb} \\
600 \mathrm{mb} \\
\text { mixed } \\
\text { surface }\end{array}$ & $\begin{array}{l}1.48 \\
0.48 \\
0.17 \\
0.16 \\
\end{array}$ & \begin{tabular}{l|}
0.91 \\
0.23 \\
0.08 \\
0.08 \\
\end{tabular} & $\begin{array}{l}0.82 \\
0.05 \\
0.01 \\
0.01 \\
\end{array}$ & \begin{tabular}{l|}
0.50 \\
0.20 \\
0.06 \\
0.06 \\
\end{tabular} & \begin{tabular}{l|}
0.89 \\
0.30 \\
0.12 \\
0.11 \\
\end{tabular} & \begin{tabular}{l|}
1.16 \\
0.43 \\
0.14 \\
0.14 \\
\end{tabular} & $\begin{array}{l}1.77 \\
0.56 \\
0.20 \\
0.18 \\
\end{array}$ & $\begin{array}{l}4.95 \\
1.32 \\
0.60 \\
0.54 \\
\end{array}$ \\
\hline Ferraz & $\begin{array}{c}300 \mathrm{mb} \\
600 \mathrm{mb} \\
\text { mixed } \\
\text { surface } \\
\end{array}$ & $\begin{array}{l}0.84 \\
0.29 \\
0.10 \\
0.10 \\
\end{array}$ & $\begin{array}{l}0.39 \\
0.15 \\
0.07 \\
0.06 \\
\end{array}$ & $\begin{array}{l}0.15 \\
0.02 \\
0.00 \\
0.00 \\
\end{array}$ & $\begin{array}{l}0.29 \\
0.11 \\
0.03 \\
0.03 \\
\end{array}$ & $\begin{array}{l}0.52 \\
0.17 \\
0.05 \\
0.05 \\
\end{array}$ & \begin{tabular}{l|}
0.72 \\
0.23 \\
0.09 \\
0.08 \\
\end{tabular} & $\begin{array}{l}1.15 \\
0.39 \\
0.14 \\
0.13 \\
\end{array}$ & $\begin{array}{l}1.78 \\
0.74 \\
0.31 \\
0.30 \\
\end{array}$ \\
\hline
\end{tabular}


EULERIAN GRANTOUR - LLNL JJA

C: Mixed Layer

RADON-222 Mixing Ratio [*e21 v/v]

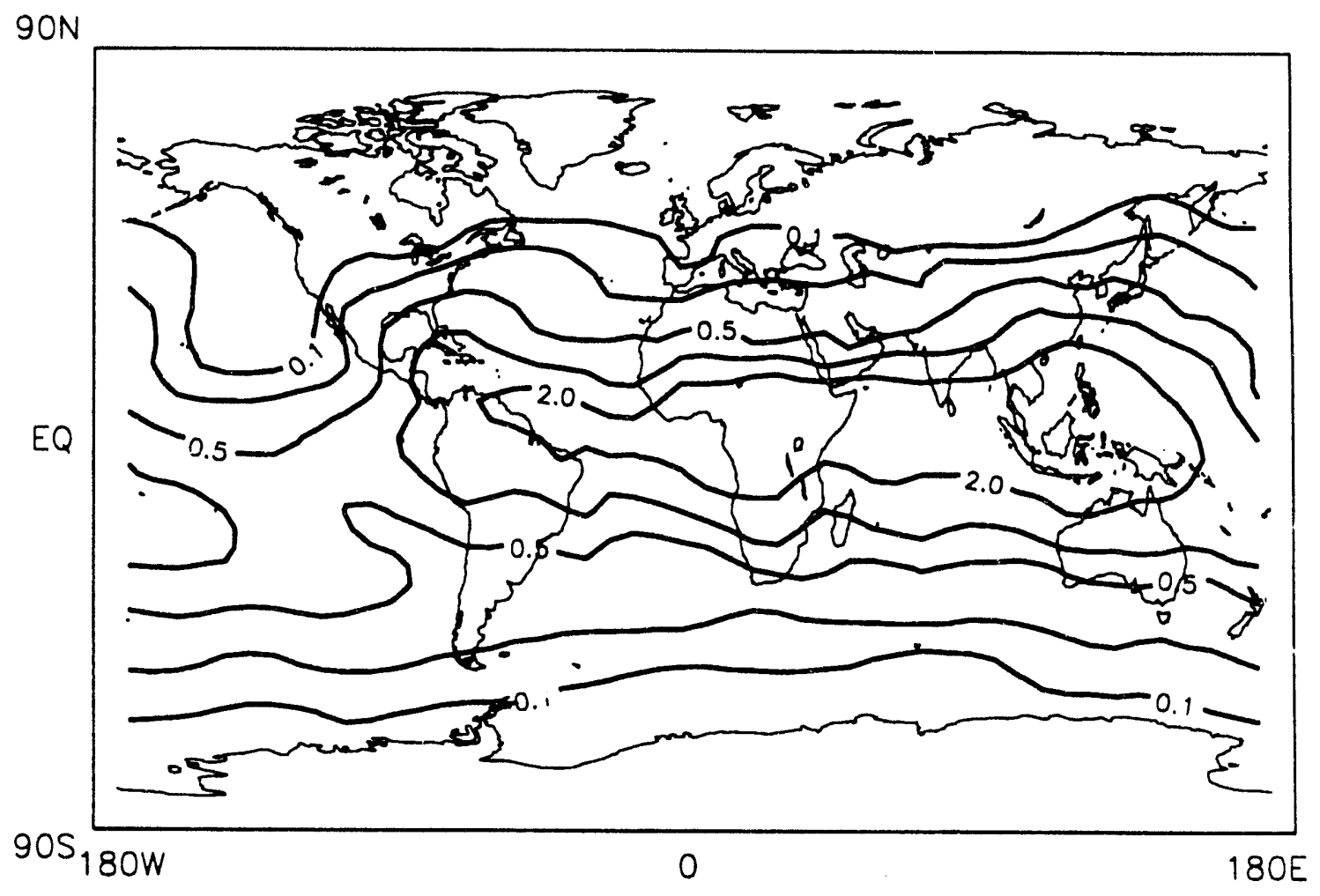


EULERIAN GRANTOUR-LLNL

\section{C: $600 \mathrm{mb}$}

$$
\text { RADON-222 Mixing Ratio [*e21 v/v] }
$$

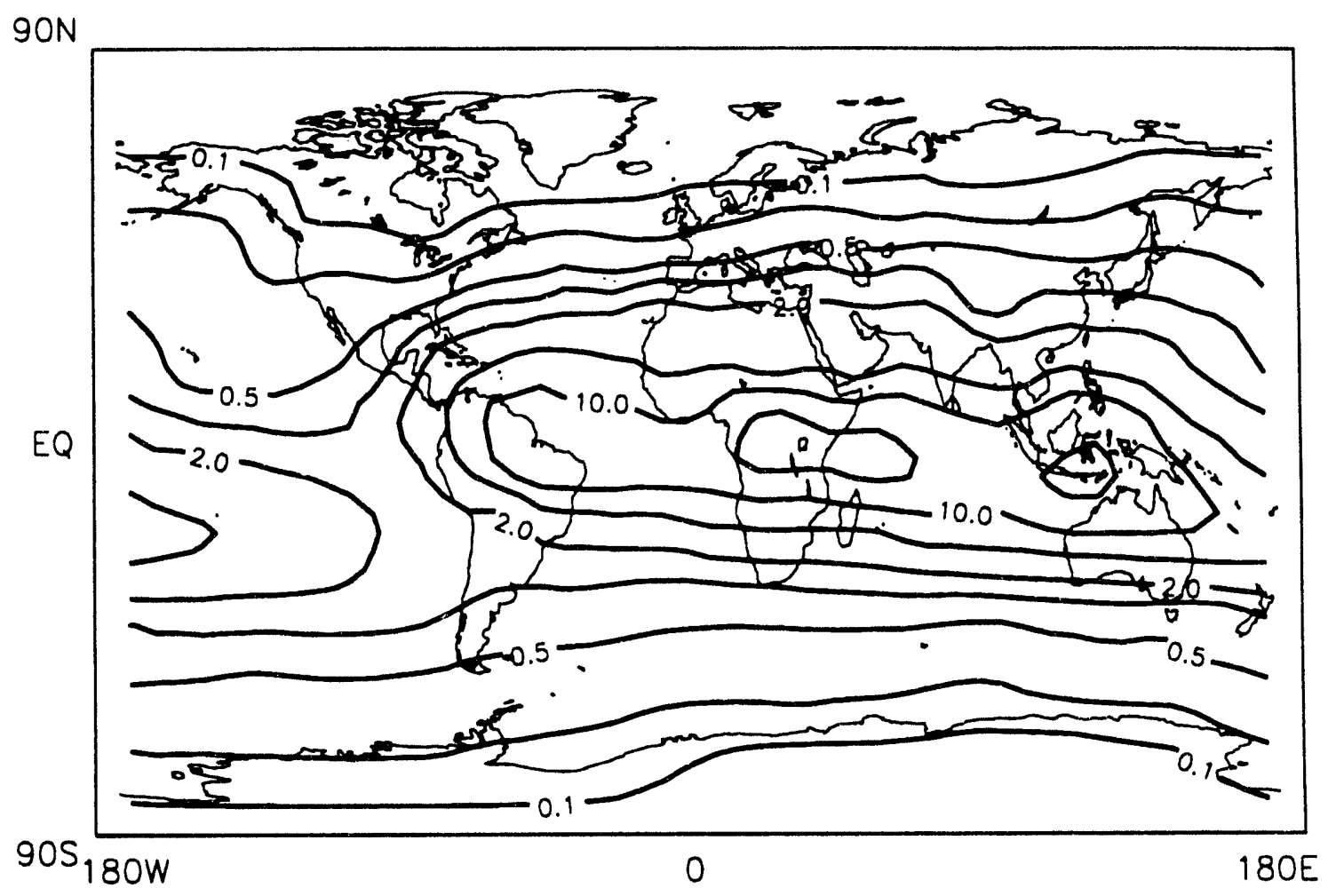


EULERIAN GRANTOUR-LLNL

\lrcorner$\lrcorner A$

\section{C: $300 \mathrm{mb}$}

$$
\text { RADON-222 Mixing Ratio [*e21 v/v] }
$$

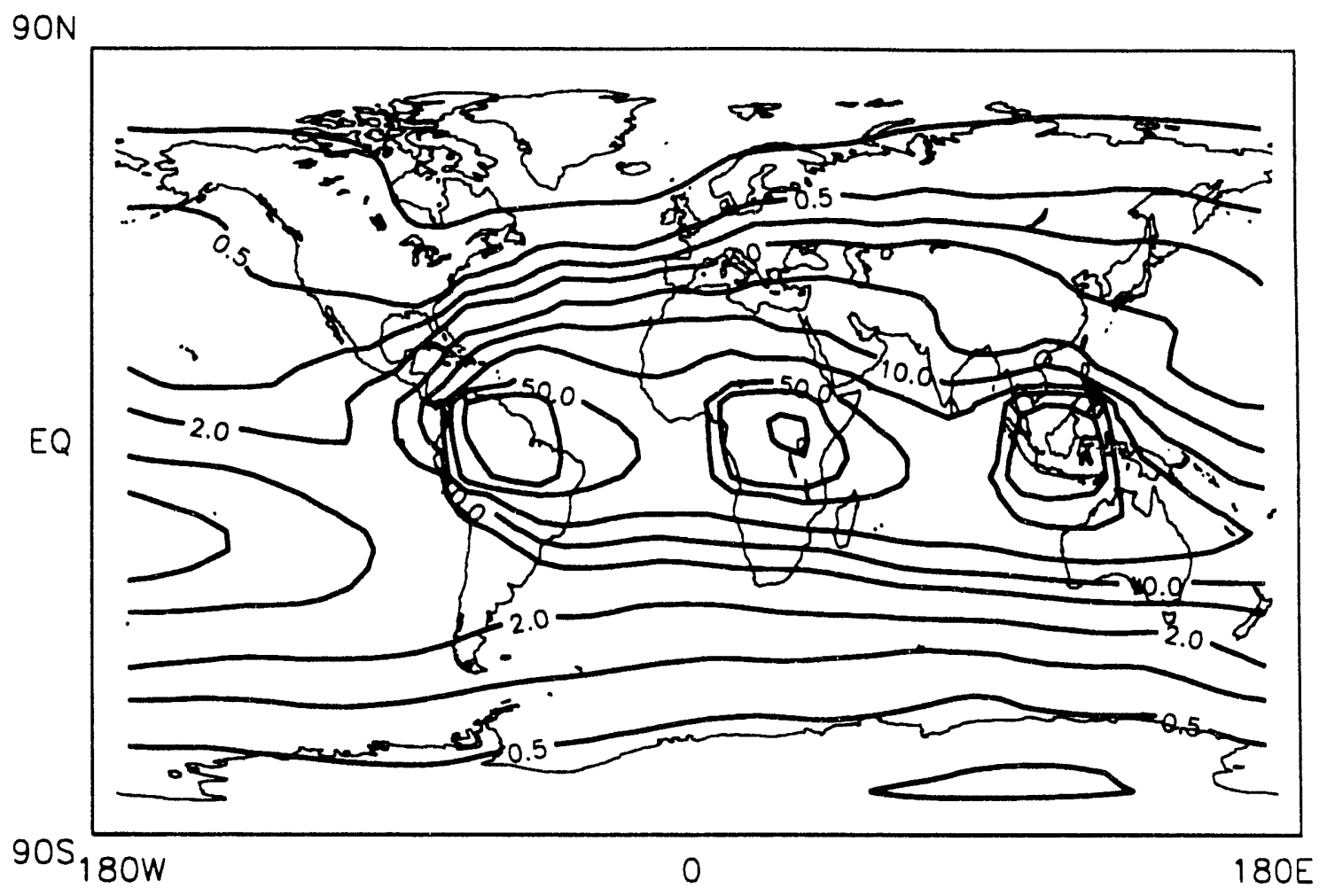


EULERIAN GRANTOUR-LLNL

JJA

C: Zonal Avg

RADON-222 Mixing Ratio [*e21 v/v]

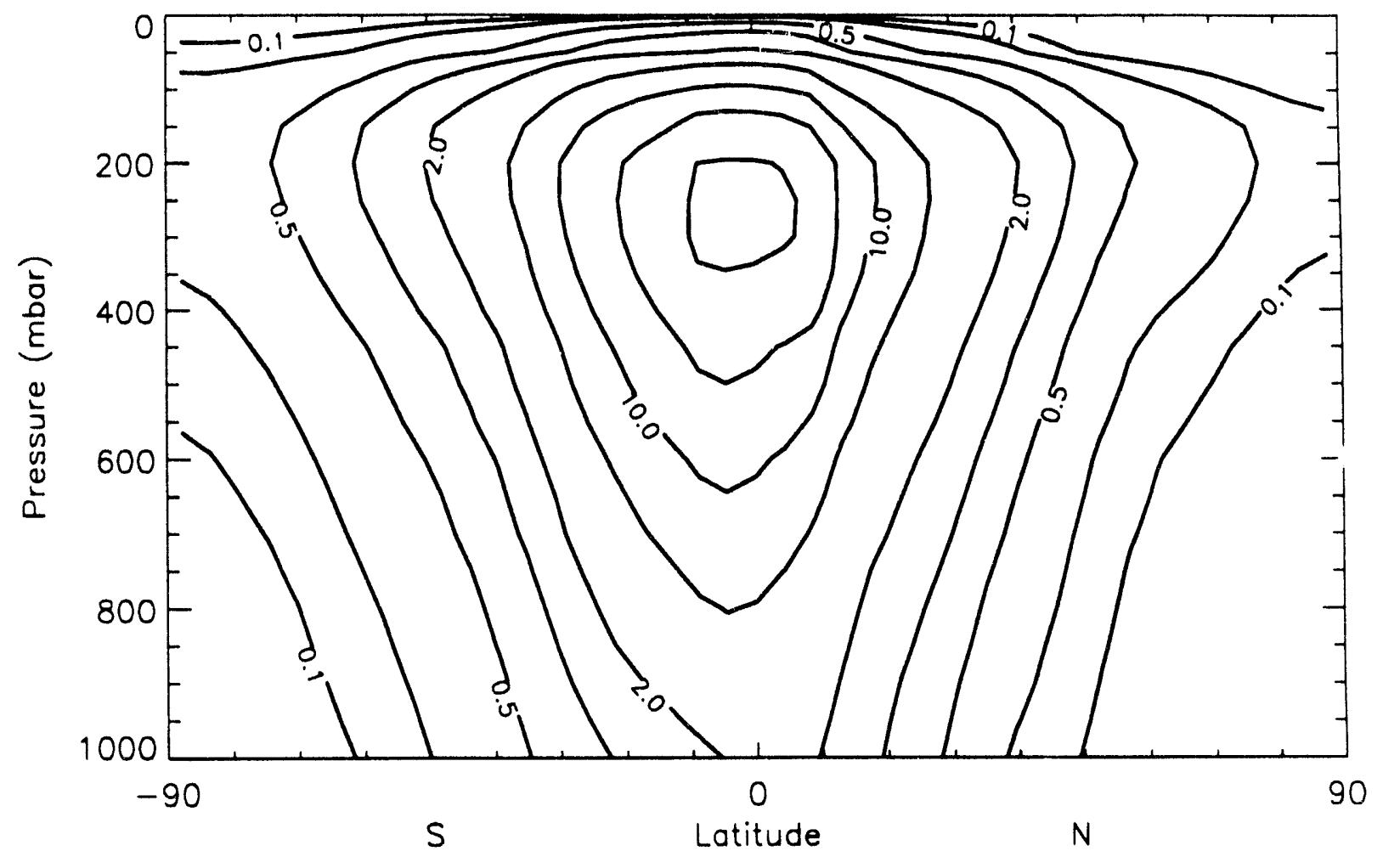


EULERIAN GRANTOUR-LLNL

JJA

C: OEW

RADON-222 Mixing Ratio [*e21 v/v]

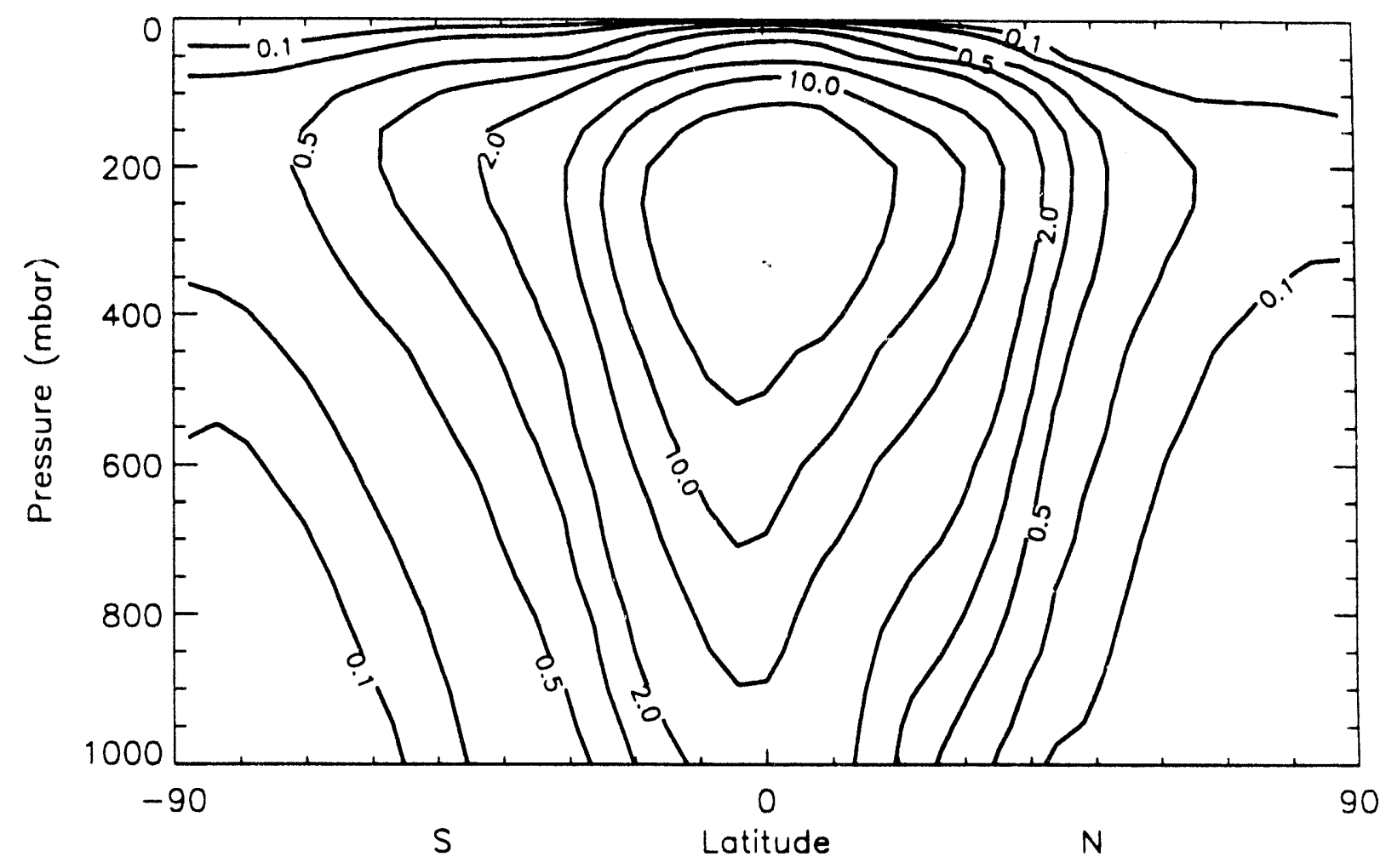


EULERIAN GRANTOUR-LLNL

JJA

\section{C: $180 E W$}

\section{RADON-222 Mixing Ratio [*e21 v/v]}

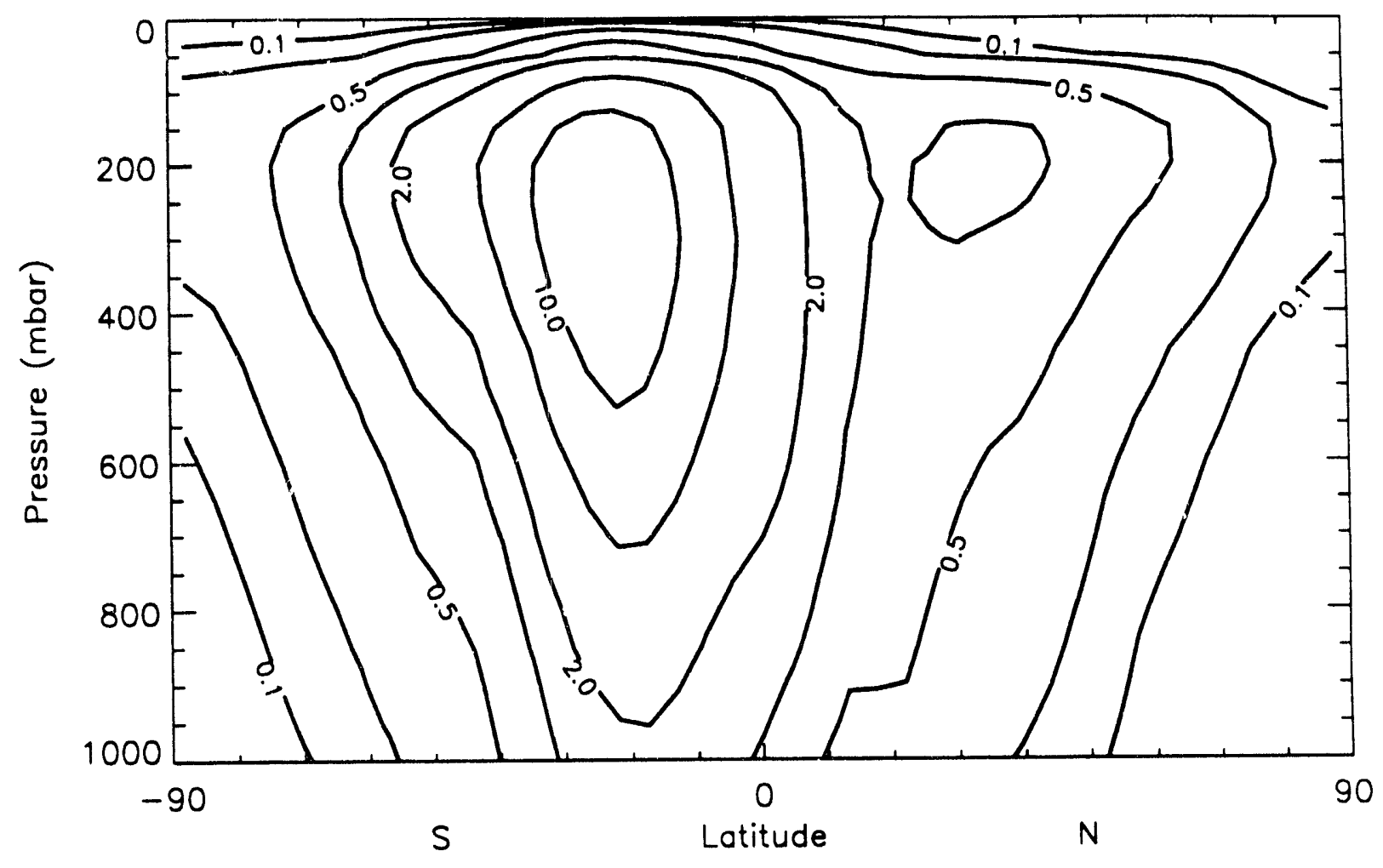




\section{APPENDIX A}

Specifications for the three radon simulations 
From pratherehalo.ps.uci.edu Thu sep $2317: 03: 421993$

Date: Fri, 24 Sep 93 00:11:03 GMT

From: Michael Prather <pratherchalo.ps.uci.edu>

To: Janedscuba.1lnl gov (Jane Dignon)

Subject: Re: WCRP '93 workshop

Content-Length: 14665

observations still being prepared

comparison not done model-by-model, but across all models by tooplc,

michael

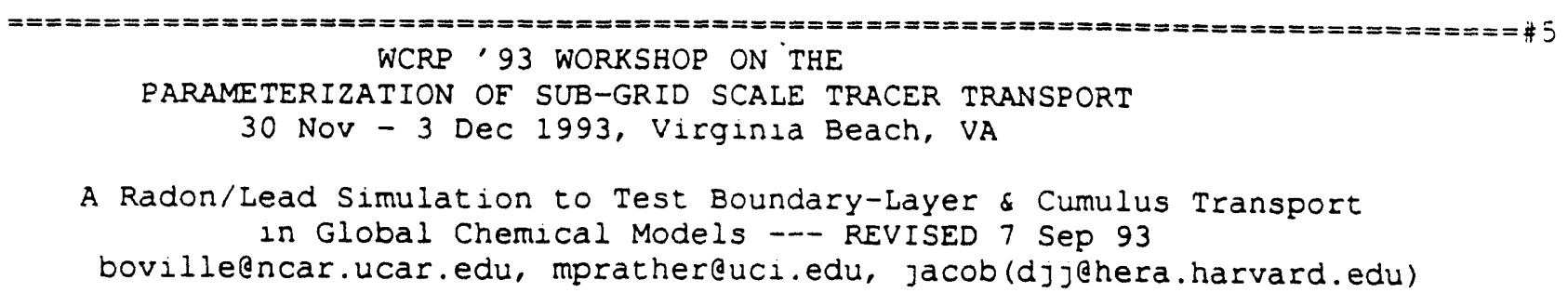

A Radon/Lead Simulation to Test Boundary-Layer \& Cumulus Transport in Global Chemical Models -- REVISED 7 Sep 93

bovilleencar.ucar.edu, mprather@uci.edu, jacob(djjehera.harvard.edu)

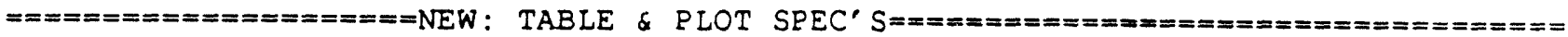
SEE LAST PAGES

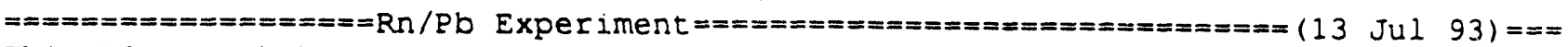
This WCRP workshop includes tutorlals and discussion about how to represent subgrid-scale transport of trace species in global models (i.e., boundary exchange between surface \& free troposphere and cloud convection; transport of water vapor, heat, reactive and unreactive chemicals). This writeup defines the numerical simulation of 222Rn (and the extension to 210Pb).
\end{abstract}

The PRIMARY GOAL of this workshop is to develop global chemical transport models (CTMs) in conjunction with numerical weather prediction models and general circulation models of the atmosphere. Attendance at the workshop will be limited, and preference will be given to those groups developing high resolution 4-D (i.e., spatial and temporal) CTMs. This goal is met by the tutorials, discussions with the NWP modelers, and the Case A simulation of radon and lead deposition compared with observations.

A SECONDARY GOAL of this workshop is to understand how current cTMs transport short-lived trace species. The key element in model predictions of chemical change in the troposphere (i.e., ozone, $O H$ ) is the distribution of tropospheric NOx (=NO+NO2). NOx is short-lived (hours to days), has highly non linear chemistry, and has a wide variety of sources whose roles are not yet guantified. Cases $B-C$ of the radon simulations are intended to give a standardized "firstlook" at atmospheric distributions predicted for different source terms known to be important in the global Nox budget:

$A=$ surface (natural radon), B=aircraft, $C=1$ ightning

This 2nd goal of the workshop is important to IPCC Climate Assessments, and will provide a necessary yardstick for interpreting the different model predictions of tropospheric chemistry that hinge on Nox distributions from different sources. Current CTMs include 2-D and coarse 3-D (e.g.. monthly averaged) models, and we anticipate representation of some of these models at the Workshop.

SOURCE: $\quad$ TOTAL $=16 \mathrm{~kg} / \mathrm{Yr}$ of $222 \mathrm{Rn}$

Case A - radon is emitted ubiquitously by decay of radium in soils: 70 - 90 degrees latitude $(\mathrm{N} \& \mathrm{~S})$ : no emissions (all surfaces) 


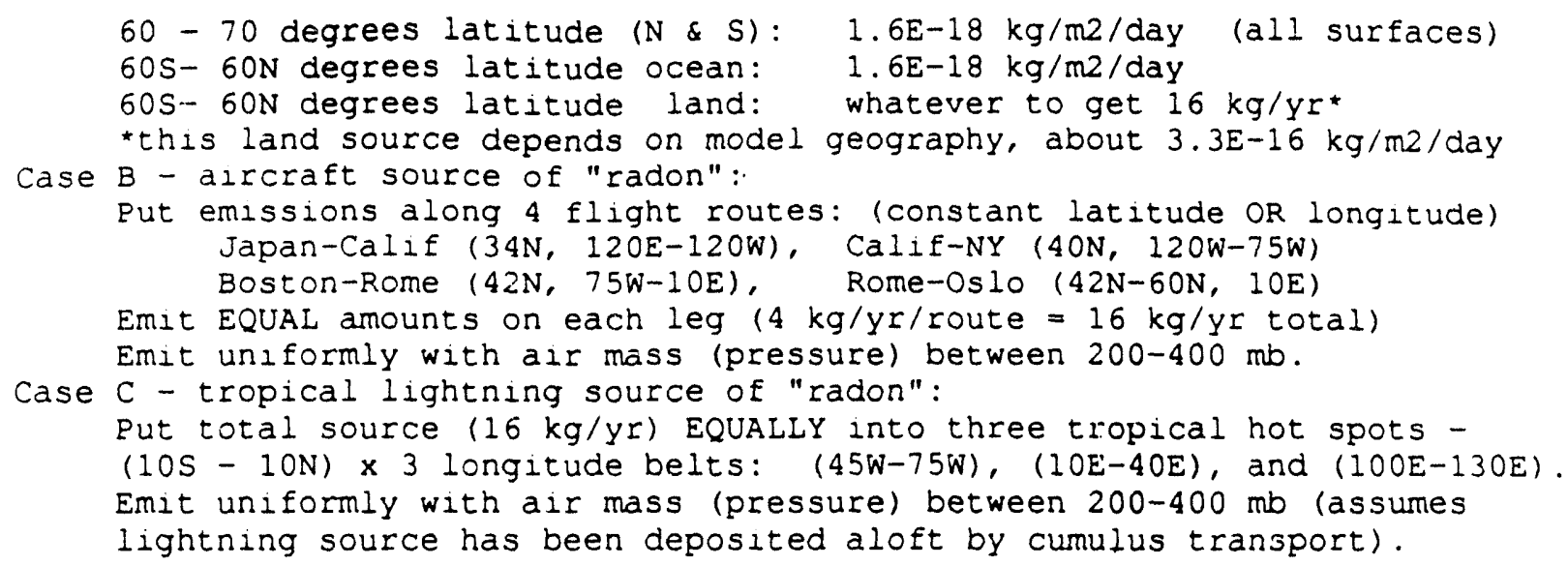


CHEMISTRY :

Remove $R$ n with a constant loss frequency of $2.10 \mathrm{E}-6 / \mathrm{sec}$ (about 5.5 days)

* Case A only** assume each 222Rn loss = instantaneous production of 210Fb. Apply a wet deposition sink to $210 \mathrm{~Pb}$ using any scheme by which a highly water-soluble, submicron aerosol is removed in your model. Include a dry deposition velocity of $0.1 \mathrm{~cm} / \mathrm{sec}$ (referenced to $10 \mathrm{~m}$ altitude) for $210 \mathrm{pb}$ over all surfaces.

N.B. The focus of this workshop is on transport of radon, the lead experiment is included as an example of possible directions for future workshops. Therefore it is not so constrained as the radon experiment, and we leave the $\mathrm{Pb}$ results for an open discussion at the end of the workshop, comparing approaches on heterogeneous removal processes.

INITIALIZE with near-zero levels of $R \cap$ and $P b$ on NOV 1 and on MAY 1, RUN for 4 months, REPORT statistics for final 3: DEC-JAN-FEB \& JUN-JUL-AUG.

UNITS: at $16 \mathrm{~kg} / \mathrm{yr}$, the global mean mixing ratio is thus $6.5 \mathrm{E}-21 \mathrm{v} / \mathrm{v}$, thus report all concentrations as mixing ratios in units of $1 \mathrm{E}-21 \mathrm{v} / \mathrm{v}$.

PERIOD: all statistics (means, rms, medians, q-tiles, fluxes) based on the full three month period June 1 - August 31 and December 1 - Eeb 28.

MODEL DIAGNOSTICS \& COMPARISON with MEASUREMENTS:

222Rn DIAGNOSTICS (Cases A-D, except surface times series for Case A only):

3-Month (!) averages or times series requested for both $D J F$ and JJA

(1) At the following geographic sites, record modeled time series of radon:

8 SITES:

a Kirov, Russia 58N 49E "mixed" layer $=300 \mathrm{~m}$ above surface

b Cincinnati, Ohio $40 \mathrm{~N} 84 \mathrm{~W} \quad 600 \mathrm{mb}$ (true pressure, not sigma)

c Socorro, N.Mexico 34N $107 \mathrm{~W}$

d Hawail $* * * 20 \mathrm{~N} 155 \mathrm{~W}$

e Samoa *** 14S $171 \mathrm{~W}$

f Crozet Isl.***463 51E

1 PLOT per site \& season, 3 altitude on page): time series, sub-select surface e $1400 \mathrm{H}$

TABULATED values (site $x$ season $x$ alt)

$g$ Ferraz, Antarct $62558 \mathrm{~W}$ means, rms, min, 1/4, median, 3/4, max

(2) latitude $x$ pressure (linear) of $v / v$-month means at DATELINE (180EW) and GREENWICH (OEW), and $v / v$ 3-month zonal mean - [THREE PLOTS per season].

(3) latitude $x$ longitude of $v / v$-month means at "mixed" layer $(300 \mathrm{~m})$, at $600 \mathrm{mb}$ and at $300 \mathrm{mb}-$

[THREE PLOTS per season].

$210 \mathrm{~Pb}$ DIAGNOSTICS (Case A only):

(4) latitude $x$ pressure (linear) of $v / v$-month means at DATELINE (180EW) and GREENWICH (OEW), and $v / v$ 3-month zonal mean - [THREE PLOTS per season].

(5) table of amount of $\mathrm{Pb}(\mathrm{kg})$ : (a) total in atmosphere, (b) $\mathrm{p}<600 \mathrm{mb}$ (altitudes above), and (c) $p<300 \mathrm{mb}$ integrated over all latitudes \& longitudes at the end of each of 3-month simulation (Feb $28 \&$ Aug 31 ).

THE MEASUREMENTS (Rn/Pb) TO BE COMPARED WITH WILL BE DEVELOPED OVER THE SUMMER, AND THESE "DATA" PLOTS WILL BE SENT OUT TO ALL WHO CONTRIBUTE TO THE WORKSHOP BY GETTING THEIR RESULTS (PLOTS \& TABLES) IN TO THE ORGANIZERS.

RESULTS DUE in by 1 NOV 93

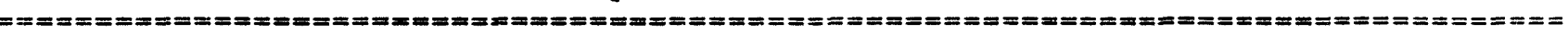

WE PLAN TO HAVE 'TOPICAL' DISCUSSION LEADERS WHO WILL TAKE A SPECIFIC

DIAGNOSTIC EROM ALL PARTICIPATING MODELS AND CROSS-COMPARE. 
Sep $2408: 521993$ standard input Page 4

THIS IS THE TYPE MODEL COMPARISONS DONE FOR STRATOSPHERE OVER THE

PAST SEVERAL YEARS. THEREFORE, PLEASE SUBMIT COPIES OF YOUR FIGURES

AND TABLES IN THE FORMAT (INCLUDING SCALE) DESCRIBED BELOW.

ALSO, PLEASE BRING TRANSPARENCIES (ONE SET) TO THE MEETING, WE WILL NOT BE ABLE TO MAKE THAT MANY TRANSPARENCIES AT THE MEETING.

MANY OF YOU WILL BE ASKED TO LEAD THE COMPARISON ON A SPECIEIC TOPIC:

e.g. REMOTE SITES, FREE TROP, LAT/LONG GRADIENTS, TIME SERIES,... 
Sep $2408: 521993$ standard input Page 5
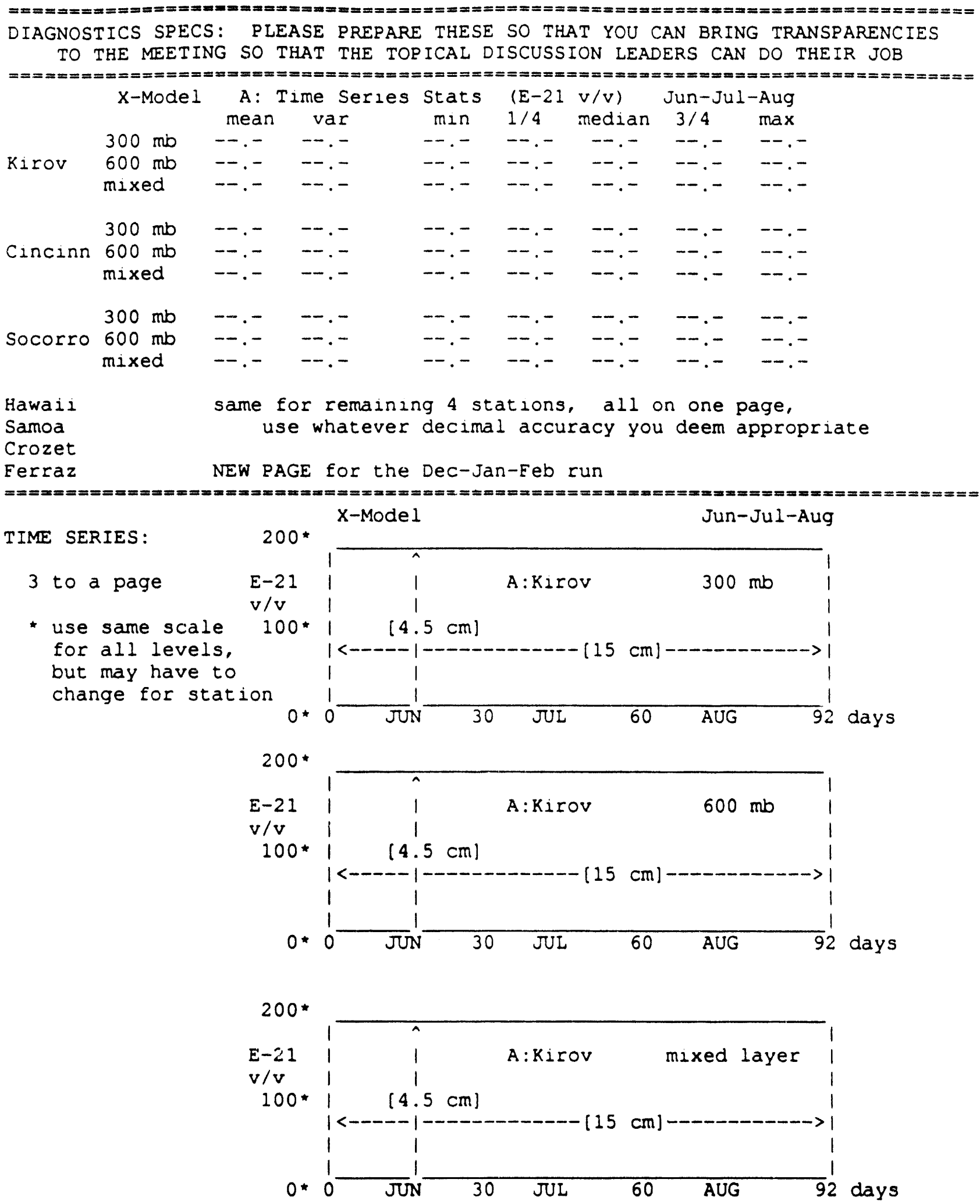
,

Sep $2408: 521993$ standard input Page 6

and the same for Dec-Jan-Feb (NEW PAGE) 


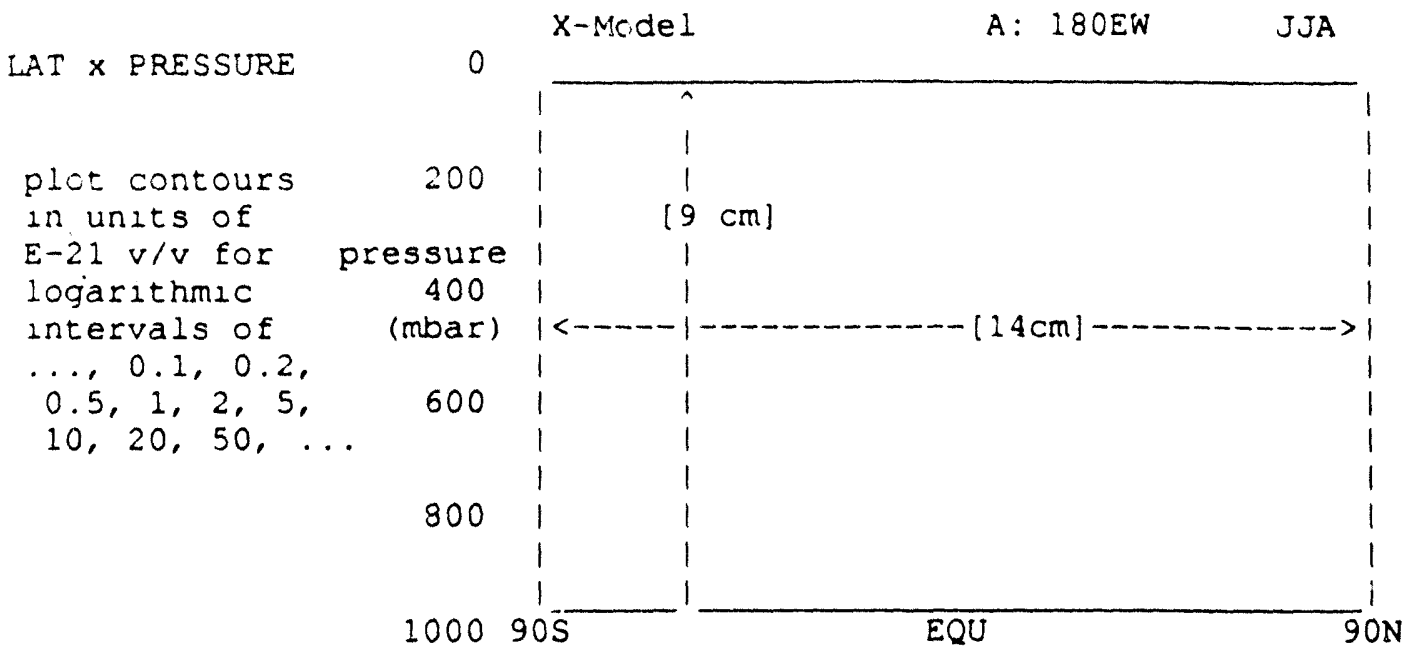

LAT $\times$ LONGITUDE
X-Mode 1
A : SURFACE
JJA

plot contours

in units of

E-21 $\mathrm{v} / \mathrm{v}$ for

logarithmic

intervals of

$\ldots, 0.1,0.2$,

$0.5,1,2,5$,

$10,20,50,100$,

$200, \ldots$

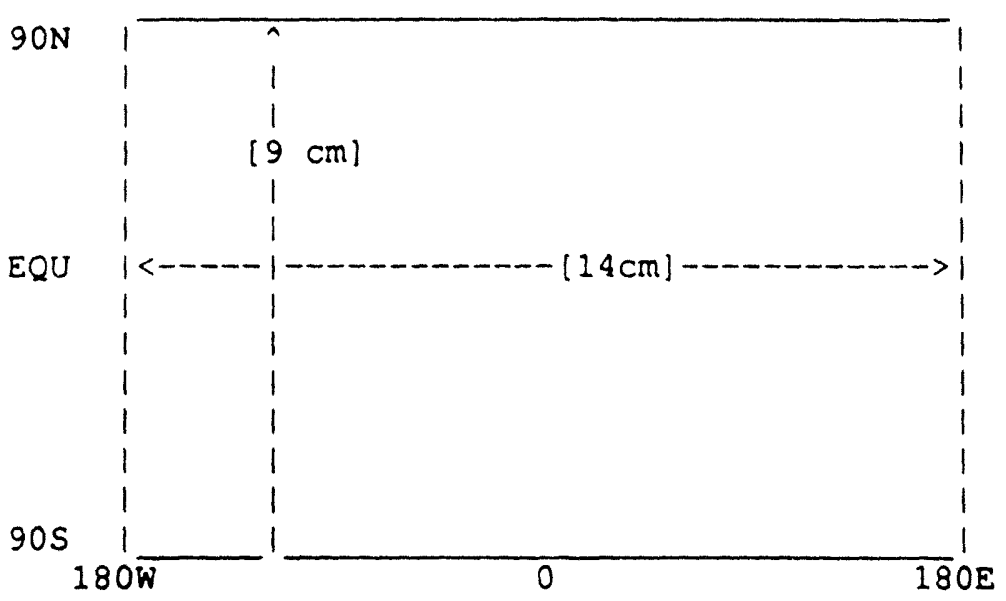

WE NEED TO BE ABLE TO PLAN A BALANCE BETWEEN THE DIFFERENT

SCIENTIEIC GROUPS NEEDED FOR A SUCCESSFUL WORKSHOP.

SO PLEASE SEND IN A NOTICE OF INTENTION TO PARTICIPATE IN

THE MODEL COMPARISON AS SOON AS POSSIBLE TO

Byron Boville and myself with copy to

WCRP: V.Savtchenko, WCRP c/O WMO, Cass Postale 2300 ,

CH-1211 Geneva 2, Switzerland, fax=41 227340357

IF YOU DID NOT RECEIVE THE WCRP ANNOUNCEMENT, PLEASE EAX V.SAVTCHENKO

INCLUDE: name / address / tele \& fax * / e-mail and the

reason for attendance (abstract or description). 
Appendix B

Differences between the Lagrangian and Eulerian Grantour simulations 


\section{Alist of the minor differences between the implementation of the Lagrangian Grantour (LG) radon simulations and the Eulerian Grantour (EG) radon simulations.}

1) For case A: (natural radon) LG injects radon between sigma coordinates

0.9 to 1.0 while EG injects between sigma coordinates 0.926 and 1.0 .

2) For case B: (aircraft) LG injects according to the specifications but in a corridor with width of five degrees. EG injects between sigma levels of 0.165 and 0.355 rather than between 200 and $400 \mathrm{mb}$. Horizontally the EG injections are into the following grid boxes:

\begin{tabular}{|c|c|c|c|}
\hline \multicolumn{2}{|c|}{ Specifications } & \multicolumn{2}{c|}{ EG Approximation } \\
\hline longitude & latitude & longitude & latitude \\
\hline $120 \mathrm{E}-120 \mathrm{~W}$ & $34 \mathrm{~N}$ & $120 \mathrm{E}-120 \mathrm{~W}$ & $32.9-37.3$ \\
\hline $75 \mathrm{~W}-10 \mathrm{E}$ & $42 \mathrm{~N}$ & $75 \mathrm{~W}-7.5 \mathrm{E}$ & $41.7-46.1$ \\
\hline $120 \mathrm{~W}-75 \mathrm{~W}$ & $40 \mathrm{~N}$ & $120 \mathrm{~W}-75 \mathrm{~W}$ & $37.3-41.7$ \\
\hline $10 \mathrm{E}$ & $42 \mathrm{~N}-60 \mathrm{~N}$ & $7.5 \mathrm{E}-15 \mathrm{E}$ & $41.7-59.2$ \\
\hline
\end{tabular}

3) For case C: (lightning) LG injects according to the specifications. EG injects between sigma levels of 0.165 and 0.355 rather than between 200 and $400 \mathrm{mb}$. Horizontally the EG injections are into the following grid boxes:

\begin{tabular}{|c|c|c|c|}
\hline \multicolumn{2}{|c|}{ Specifications } & \multicolumn{2}{c|}{ EG Approximation } \\
\hline longitude & latitude & longitude & latitude \\
\hline $45 \mathrm{~W}-75 \mathrm{~W}$ & $10 \mathrm{~S}-10 \mathrm{~N}$ & $45 \mathrm{~W}-75 \mathrm{~W}$ & $11 \mathrm{~S}-11 \mathrm{~N}$ \\
\hline $10 \mathrm{E}-40 \mathrm{E}$ & $10 \mathrm{~S}-10 \mathrm{~N}$ & $7.5 \mathrm{E}-37.5 \mathrm{E}$ & $11 \mathrm{~S}-11 \mathrm{~N}$ \\
\hline $100 \mathrm{E}-130 \mathrm{E}$ & $10 \mathrm{~S}-10 \mathrm{~N}$ & $97.5-127.5$ & $11 \mathrm{~S}-11 \mathrm{~N}$ \\
\hline
\end{tabular}

4) LG biases all its injections by surface pressure. In areas of high local surface pressure one expects to find more parceis, thus there is a slightly larger source term there. In areas of low surface pressure, one expects to find fewer parcels, thus there is slightly reduced injection there. The total amount injected is correct.

5) LG reports results every 12 hours. EG reports results every 24 hours.

6) Data from LG labeled "mixed layer" is actually at $977 \mathrm{mb}$. At locations where the surface pressure is less than $977 \mathrm{mb}$, the surface values are reported. Data from EG labeled "mixed layer" is actually at 0.965 in sigma coordinates. 

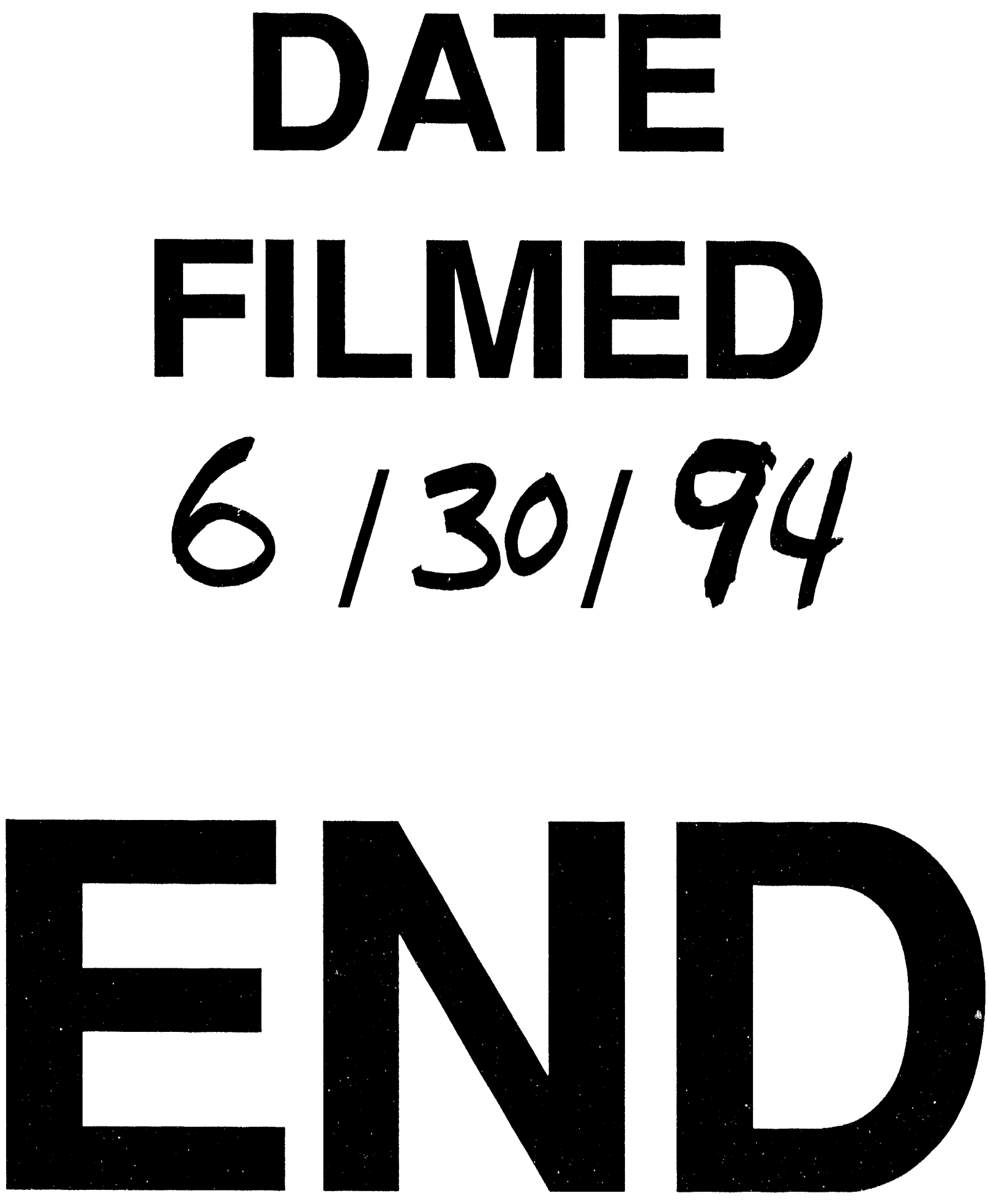
